\title{
Molecular responses to whole-body dehydration in a sequenced vertebrate, Xenopus laevis: Regulation of antioxidants and metabolism by the Sirtuin protein deacetylases
}

\section{Bryan Edward-Dinh Luu}

\section{M.Sc., B.Sc. Carleton University}

A Thesis Submitted to the Faculty of Graduate Studies and Research In partial fulfillment of the requirements for the degree of

\author{
Doctor of Philosophy \\ Department of Biology
}

Carleton University,

Ottawa, Ontario, Canada

CCopyright 2018

Bryan E Luu 
The undersigned hereby recommend to the Faculty of Graduate Studies and Research acceptance of this thesis

\section{Molecular responses to whole-body dehydration in a sequenced vertebrate, Xenopus laevis: Regulation of antioxidants and metabolism by the Sirtuin protein deacetylases}

Submitted by

\section{Bryan Edward-Dinh Luu,}

B.Sc., M.Sc.

In partial fulfillment of the requirements for the degree of Doctor of Philosophy

Chair, Department of Biology

Thesis Supervisor

External Examiner

Carleton University 


\begin{abstract}
Whole-body dehydration in the African clawed frog, Xenopus laevis, increases hematocrit and blood viscosity, which restrains oxygen delivery. This causes the resting heart rate, differences in arterio-venous blood oxygen contents, and whole-animal lactate to increase.
\end{abstract}

I hypothesized that dehydration involves changes in cellular signaling through alterations of protein posttranslational acetylation, which can increase antioxidants and regulate metabolism. Seven Sirtuin (Sirt) protein deacetylases were profiled at the mRNA level with RT-qPCR in 6 tissues (liver, muscle, heart, kidney, brain, and lung) of $X$. laevis under control versus dehydration conditions. At least some sirt transcripts increased in all tissues except for kidney and brain. Similarly, global Sirt activity assays found that Sirt deacetylase activity increased in liver, muscle, heart, and lung. Western blots revealed the relative levels of Ac-SOD2. Results showed that acetylated SOD2 decreased with whole-body dehydration in the lung, heart, and kidney, suggesting that Sirt3 deacetylase activity is triggered by dehydration to activate antioxidant activity in these tissues.

Sirt/PGC-1 $\alpha /$ FoxO-mediated upregulation of antioxidants was investigated in lung and brain of $X$. laevis. Results showed upregulations of these three controllers of antioxidants in lung (but not brain) during dehydration, as evidenced by analyses at the mRNA, protein, and phospho-protein levels. Results suggested that dehydration-induced antioxidant upregulation in $X$. laevis was mediated by Sirts, in addition to PGC-1a and the FoxO1/3 transcription factors in a tissue-specific manner. Antioxidant capacity assays 
showed that lung sustained a decrease in antioxidant capacity during dehydration, which suggests that the Sirt/PGC- $1 \alpha /$ FoxO response may be a compensatory one to restore antioxidants levels.

In the liver, muscle, and heart, PGC- $1 \alpha$ and Hif- $1 \alpha$ were assessed for their roles in activating ureagenesis, angiogenesis, and remodelling of the metabolism. MEF2mediated PGC-1 $\alpha$ upregulation occurred in the liver, but not the muscle or heart, whereas Hif- $1 \alpha$ increased in all 3 tissues with dehydration. Relative mRNA levels of genes related to glucose metabolism, angiogenesis, ureagenesis and $\beta$-oxidation were found to be differentially regulated in response to dehydration. Together, the results suggest that PGC- $1 \alpha$ and Hif- $1 \alpha$ are modulating gene expression during dehydration to suppress $\beta$ oxidation in favour of glycolysis, while ureagenesis and angiogenesis are promoted in liver. 


\section{ACKNOWLEDGEMENTS}

I would like to thank my thesis supervisor, Dr. Ken Storey, for sharing unique insights with me on how the world functions, both inside and outside of the world of science. I have grown to become who I am today due to the experiences that I have received over the past decade here. I am certain that the skills and experiences that I have gained here will put me at a significant advantage for whatever endeavours I pursue. An equal amount of thanks go to Jan Storey, who has helped me significantly improve my writing - from manuscripts to grants. I will always remember the fun Summer / Christmas Storey lab BBQ parties, and the lab trips to Upper Canada Village, Gatineau Park, and the Sugar Bush.

Lastly, I would like to thank my family, who have always supported me. 


\section{TABLE OF CONTENTS}

$\begin{array}{ll}\text { Title page } & \text { i }\end{array}$

Acceptance sheet

$\begin{array}{lll}\text { Abstract } & \text { iii }\end{array}$

Acknowledgements $\quad$ V

Table of Contents $\quad$ vi

List of Abbreviations vii

$\begin{array}{ll}\text { List of Figures } & \text { ix }\end{array}$

List of Tables $\quad$ xiv

List of Appendices $\quad$ XV

$\begin{array}{lll}\text { Chapter } 1 & \text { General introduction } & 1\end{array}$

Chapter $2 \quad$ Characterization of Sirtuins 41

Chapter $3 \quad$ Antioxidant upregulation by Sirt/PGC-1 $\alpha / \mathrm{FoxO} \quad 85$

Chapter $4 \quad$ PGC-1 $\alpha$ and Hif-1 $\alpha$-mediated remodelling of metabolism 123

$\begin{array}{lll}\text { Chapter 5 } & \text { General discussion }\end{array}$

$\begin{array}{ll}\text { Appendices } & 187\end{array}$ 


\section{LIST OF ABBREVIATIONS}

\begin{tabular}{l} 
ABTS \\
BLAST \\
CPS1 \\
cpt1 \\
DTT \\
EDTA \\
ERK \\
FoxO1 \\
FoxO3 \\
gapdh \\
glut4 \\
GSH \\
GSSH \\
HEPES \\
HDAC \\
Hif-1 $\alpha$ \\
HK \\
hnf3b \\
hnf4a \\
HO-1 \\
KCL \\
lcad \\
LDH \\
MAPK \\
MAPKK \\
MAPKKK \\
mcad \\
MEF2 \\
MnSOD/SOD2 \\
NCBI \\
Nrf2 \\
OTC \\
pdk \\
pfk \\
PGC-1 $\alpha$ \\
PGC-1乃 \\
PK \\
POS \\
ppara \\
ROS \\
RT-qPCR \\
\hline
\end{tabular}

2,2'-Azino-di-[3-ethylbenzthiazoline sulphonate] basic local alignment search tool carbamoyl phosphate synthetase 1 carnitine palmitoyltransferase 1 dithiothreitol ethylenediaminetetraacetic acid extracellular signal-regulated kinase forkhead box class $\mathrm{O}$ type 1 forkhead box class $\mathrm{O}$ type 3

glyceraldehyde-3-phosphate dehydrogenase glucose transporter 4 glutathione oxidized glutathione 4-(2-hydroxyethyl)-1-piperazineethanesulfonic acid histone deacetylase hypoxia-inducible factor-1 alpha hexokinase hepatocyte nuclear factor 3 beta hepatocyte nuclear factor 4 alpha heme oxygenase-1 potassium chloride long chain acyl-CoA dehydrogenase lactate dehydrogenase mitogen-activated protein kinase MAPK kinase MAPKK kinase medium chain acyl-CoA dehydrogenase myocyte enhancer factor- 2 manganese-dependent superoxide dismutase National Center for Biotechnology Information nuclear factor-like 2

ornithine transcarbamylase pyruvate dehydrogenase kinase phosphofructokinase peroxisome proliferator-activated receptor gamma coactivator 1-alpha Peroxisome proliferator-activated receptor gamma coactivator 1-beta pyruvate kinase preparation for oxidative stress peroxisome proliferator-activated receptor alpha reactive oxygen species ribosomal protein L27 real time-quantitative polymerase chain reaction 
scad

Sirt

SOD1

STAT

vegf small chain acyl-CoA dehydrogenase sirtuin

superoxide dismutase 1

signal transducer and activators of transcription vascular endothelial growth factor 


\section{LIST OF FIGURES}

Figure 1.1. Whole-body dehydration in X. laevis results in a diverse set of responses that help the animal cope with impaired oxygen transport due to reduced plasma volume and increased hematocrit. The brain maintains the highest priority for blood circulation, next to other vital organs, and with skeletal muscle ranked last. Evidence suggests that metabolism shifts towards anaerobic glycolysis during dehydration as whole animal lactate increases at the higher stages of dehydration.

Figure 2.1. Sirtuin mechanism of reaction.

Figure 2.2. Relative mRNA expression of 7 sirt genes was assessed in liver using RTqPCR to investigate the effects of medium and high levels of whole-body dehydration of $X$. laevis. Gene expression data were standardized against $r p l 27$ as a reference gene. Data are means \pm SEM, $n=4$ independent biological replicates. Statistically significant differences compared to the control condition are denoted with an asterisk $(*)$ and were determined with a one-way ANOVA and post hoc Dunnett's test $(p<0.05)$.

Figure 2.3. Relative mRNA expression of 7 sirt genes was assessed in $X$. laevis skeletal muscle using RT-qPCR to investigate the effects of medium and high levels of wholebody dehydration. Gene expression data were standardized against rpl27 as a reference gene. Other information as in Figure 2.2.

Figure 2.4. Relative mRNA expression of 7 sirt genes was assessed in X. laevis brain using RT-qPCR to investigate the effects of medium and high levels of whole-body dehydration. Gene expression data were standardized against $r p l 27$ as a reference gene. Other information as in Figure 2.2.

Figure 2.5. Relative mRNA expression of 7 sirt genes was assessed in X. laevis heart using RT-qPCR to investigate the effects of medium and high levels of whole-body dehydration. Gene expression data were standardized against gapdh as a reference gene. Other information as in Figure 2.2.

Figure 2.6. Relative mRNA expression of 7 sirt genes was assessed in lungs using RTqPCR to investigate the effects of whole-body dehydration of $X$. laevis at high dehydration levels. Gene expression data quantified from lung tissues were standardized against $r p l 27$ as a reference gene. Other information as in Figure 2.2, except that $\boldsymbol{n}=\mathbf{6}$ independent biological replicates.

Figure 2.7. Relative mRNA expression of 7 sirt genes was assessed in kidneys using RTqPCR to investigate the effects of whole-body dehydration of $X$. laevis at high dehydration levels. Gene expression data quantified from kidneys tissues were 
standardized against $r p l 27$ as a reference gene. Other information as in Figure 2.2, except that $\boldsymbol{n}=\mathbf{8}$ independent biological replicates.

Figure 2.8. A global Sirt activity assay was used to determine the relative SIRT deacetylase activity in $X$. laevis tissues exposed to dehydration conditions. Enzyme activities from cell lysates are expressed as relative activity (arbitrary units) for normalized protein amounts. Histograms show means \pm SEM from $n=4$ independent biological replicates. Statistically significant differences between dehydrated conditions compared to the controls are denoted with an asterisk $\left(^{*}\right)$ and were determined with a one-way ANOVA and post hoc Dunnett's test $(p<0.05)$.

Figure 2.9. The acetylated lysine 68 residue on SOD2 of humans, identified by a red box, is conserved in other mammals and in X. laevis. The SOD2 K68 residue occurs in all three mammalian sequences, but is K70 in X. laevis. Primary structures of SOD2 for all animals were obtained from NCBI Protein, and were aligned with EMBL-EBI Clustal Omega. Only a segment of the full primary sequence is shown.

Figure 2.10. Immunoblots depicting relative changes in the amount of acetylated SOD2 (K70) in X. laevis tissues. Dehydration led to reduced amounts of acetylated SOD2 protein in heart, lung, and kidney tissues.

Figure 2.11. Relative abundance of $X$. laevis acetylated SOD2 (K70) protein in six tissues under control, medium, and high dehydration states. Histograms show means \pm SEM from $n=4$ independent biological replicates. Statistically significant differences between dehydrated conditions compared to the controls are denoted with an asterisk $\left(^{*}\right)$ and were determined with a one-way ANOVA and post hoc Dunnett's test $(p<0.05)$.

Figure 2.12. Roles of various Sirts in the regulation of antioxidant response signaling.

Figure 2.13. The SIRT4 and SIRT5 proteins have metabolic roles in the cell. SIRT4 is known to repress fatty acid oxidation, and SIRT5 activates the urea cycle by deacetylating and activating CPS1.

Figure 2.14. In pulmonary fibrosis, a suppression of all 7 SIRT proteins is observed, but particularly SIRT7. Suppression of SIRT7 during pulmonary fibrosis is known to facilitate an upregulation of collagen, $\alpha$-SMA, and Smad3. Oppositely, overexpression of SIRT7 in fibroblasts results in decreased collagen, $\alpha-S M A$, and Smad3 expression, which are genes that are characteristic of pulmonary fibrosis.

Figure 3.1. Immunoblotting was used to determine relative protein levels of 4 SIRT proteins known to play roles in antioxidant signaling in the lungs of $X$. laevis, comparing control, medium and high levels of whole-body dehydration. Chemiluminescent data derived from immunoblots was standardized against total protein loaded as represented by Coomassie staining of membranes. Histograms show means \pm SEM, $n=4$ independent biological replicates. Statistically significant differences compared to the control 
condition are denoted with an asterisk $(*)$ and were determined with a one-way ANOVA and post hoc Dunnett's test $(p>0.05)$.

Figure 3.2. Immunoblotting was used to determine relative protein levels of 4 SIRT proteins known to play roles in antioxidant responses in the brains of $X$. laevis comparing control, medium and high levels of whole-body dehydration. Other information as in Figure 3.1.

Figure 3.3. Relative mRNA (A) and protein (B) levels of PGC-1 $\alpha$ were assessed in the lungs and brains of $X$. laevis comparing control, medium and high levels of whole-body dehydration. Gene expression data was quantified with RT-qPCR and standardized against rpl27 as a reference gene. Chemiluminescent data from immunoblots was standardized against total protein loaded as represented by Coomassie staining of membranes. Histograms show means $\pm \mathrm{SEM}, n=4-6$ independent biological replicates.

Figure 3.4. Partial primary structures of FoxO1 and FoxO3 in X. laevis, H. sapiens, $R$. norvegicus, and M. musculus. The phosphorylated Ser256 (A) and Ser319 (B) FoxO1 residues of humans are evolutionarily conserved as Ser245 and Ser308 in X. laevis. The conserved phosphorylated Ser253 (C) on human FoxO3 protein is also conserved as Ser238 in X. laevis. These conserved phosphorylation sites are shown in red boxes.

Figure 3.5. Relative mRNA (A) and protein (B) levels of FoxO1 were assessed in the lungs and brains of $X$. laevis comparing control, medium and high levels of whole-body dehydration. Other information as in Figure 3.3.

Figure 3.6. Relative mRNA (A) and protein (B) levels of FoxO3 were assessed in the lungs and brains of $X$. laevis comparing control, medium and high levels of whole-body dehydration. Other information as in Figure 3.3.

Figure 3.7. Relative mRNA (A) and protein (B) levels of Catalase and HO-1 were assessed in the lungs and brains of $X$. laevis comparing control, medium and high levels of whole-body dehydration. Other information as in Figure 3.3.

Figure 3.8. Relative antioxidant capacity of six $X$. laevis tissues comparing control, medium and high dehydration conditions. Assay results demonstrate the capacity of tissue samples to prevent oxidation of ABTS by metmyoglobin and hydrogen peroxide. Equal protein amounts were loaded for each biological replicate. Absorbance was measured at $405 \mathrm{~nm}$ and data was interpolated against a Trolox standard curve. Histograms show means \pm SEM, $n=4$ independent biological replicates. Statistically significant differences compared to the control condition are denoted with an asterisk $(*)$ and were determined with a one-way ANOVA and post hoc Dunnett's test $(p>0.05)$. 
Figure 3.9. Relative mRNA (A) and protein (B) levels of SOD1 and SOD2 were assessed in lung and brain of $X$. laevis comparing control, medium and high levels of whole-body dehydration. Other information as in Figure 3.3.

Figure 3.10. A schematic of the antioxidant signaling response in the lungs of $X$. laevis to dehydration showing the integration of data from the present chapter and that in Chapter $\mathbf{2}$, and the established roles of the targets from in the literature.

Figure 4.1. The "canonical" PGC- $1 \alpha$ pathway. The expression and activity of PGC- $1 \alpha$ is known to be increased by Sirt and MEF2 upstream regulators. Once activated, PGC-1 $\alpha$ plays diverse cellular roles including in nitrogen and fatty acid metabolism, in addition to the regulation of antioxidants and angiogenesis.

Figure 4.2. The "canonical" Hif-1 a pathway. The Hif-1a transcription factor, similar to PGC-1 $\alpha$, is stabilized and inducible by Sirt deacetylase activity, and also regulates angiogenic genes. Unlike PGC-1 $\alpha$, Hif-1a suppresses fatty acid oxidation in favour of glucose metabolism.

Figure 4.3. Relative pgcla mRNA (A) and PGC-1 $\alpha$ protein levels (B) determined by RTqPCR and immunoblotting, respectively in the liver, muscle, and heart of $X$. laevis under control, medium, and high levels of whole-body dehydration. Gene expression data was quantified with RT-qPCR and standardized against rpl27 as the reference gene for liver and muscle, whereas gapdh was used for heart. Chemiluminescent data from immunoblots was standardized against total protein loaded as determined by subsequent Coomassie staining of membranes. Histograms show means \pm SEM, $n=4$ independent biological replicates. Statistically significant differences compared to the control condition are denoted with an asterisk $(*)$ and were determined with a one-way ANOVA and post hoc Dunnett's test $(p<0.05)$.

Figure 4.4. Relative mef $2 a$ mRNA (A), MEF2A protein (B), and MEF2A nuclear protein levels determined by RT-qPCR and immunoblotting, respectively in the liver, muscle, and heart of $X$. laevis under control, medium, and high levels of whole-body dehydration. Other information as in Figure 4.3; immunoblot images are shown in Figure 4.6.

Figure 4.5. Relative mef $2 c$ mRNA (A), MEF2C protein (B), and MEF2C nuclear protein levels determined by RT-qPCR and immunoblotting, respectively in the liver, muscle, and heart of $X$. laevis under control, medium, and high levels of whole-body dehydration. Other information as in Figure 4.3; immunoblot images are shown in Figure 4.6.

Figure 4.6. Visualization of chemiluminescent bands of immunoblots for MEF2A and MEF2C proteins used to provide quantitative data in Figures 4.4 and 4.5.

Figure 4.7. Relative mRNA levels of hepatic transcription factors $h n f 3 b$ and $h n f 4 a$ and urea cycle genes cps 1 and otc, as determined by RT-qPCR, in the liver of $X$. laevis under 
control, medium, and high levels of whole-body dehydration. Other information as in Figure 4.3.

Figure 4.8. Relative hifla mRNA (A) and Hif-1 $\alpha$ protein levels (B) determined by RTqPCR and immunoblotting, respectively, in 6 tissues of $X$. laevis under control, medium, or high levels of whole-body dehydration. Gene expression data was quantified with RTqPCR and standardized against $r p l 27$ as the reference gene for all tissues except the heart, where gapdh was used. Histograms show means \pm SEM, $n=4$ independent biological replicates for all tissues except for kidney and lung RT-qPCR analyses, which are $n=8$ and $n=6$, respectively. Other information as in Figure 4.3.

Figure 4.9. Relative mRNA levels of ppara (A) and $p g c 1 b(\mathrm{~B})$ as determined by RTqPCR, in the liver, muscle, and heart of $X$. laevis under control, medium, and high levels of whole-body dehydration. Other information as in Figure 4.3.

Figure 4.10. Relative mRNA levels of $l c a d$, mcad, and scad as determined by RT-qPCR, in the liver (A), muscle (B), and heart (C) of X. laevis under control, medium, and high levels of whole-body dehydration. Other information as in Figure 4.3.

Figure 4.11. Relative mRNA levels of $c p t 1 a$ and $c p t 1 b$ as determined by RT-qPCR, in the liver (A), muscle (B), and heart (C) of $X$. laevis under control, medium, and high levels of whole-body dehydration. Other information as in Figure 4.3.

Figure 4.12. Relative mRNA levels of $g l u t 4, h k, p f k, p d k 1, p d k 3$ and $p d k 4$ as determined by RT-qPCR, in the liver of $X$. laevis under control, medium, and high levels of wholebody dehydration. Other information as in Figure 4.3.

Figure 4.13. Relative mRNA levels of glut4, $h k, p f k, p d k 1, p d k 3$ and $p d k 4$ as determined by RT-qPCR, in the muscle of $X$. laevis under control, medium, and high levels of wholebody dehydration. Other information as in Figure 4.3.

Figure 4.14. Relative mRNA levels of glut4, $h k, p f k, p d k 1, p d k 3$ and $p d k 4$ as determined by RT-qPCR, in the heart of $X$. laevis under control, medium, and high levels of wholebody dehydration. Other information as in Figure 4.3.

Figure 4.15. Relative mRNA levels of vegf as determined by RT-qPCR, in 6 tissues of $X$. laevis under control, medium, and high levels of whole-body dehydration. Other information as in Figure 4.8. 


\section{LIST OF TABLES}

Table 1.1. A summary of physiological and molecular responses that occur with wholebody dehydration in $X$. laevis, and their implications.

Table 2.1 A summary of the characterization of Sirts and their potential roles in response to whole-body dehydration in $X$. laevis 


\section{LIST OF APPENDICES}

$\begin{array}{ll}\text { Appendix A } & \text { List of Publications } \\ \text { Appendix B } & \text { Scientific Communications at Meetings } \\ \text { Appendix C } & \text { Primer design for RT-qPCR } \\ \text { Appendix D } & \text { Assessment of RNA Integrity } \\ \text { Appendix E } & \text { Controls for Gene Quantification using RT-qPCR } \\ \text { Appendix F } & \text { Reference gene selection } \\ \text { Appendix G } & \text { Western immunoblot quantification } \\ \text { Appendix H } & \text { Antibody list } \\ \text { Appendix I } & \text { Western blot conditions } \\ \text { Appendix J } & \text { List of primers }\end{array}$




\section{CHAPTER 1}

General Introduction

"We do not truly understand how hydration affects health and well-being, even the impact of water intakes on chronic diseases."

- Barry M. Popkin, PhD "Water, Hydration and Health" 


\subsection{Introduction}

All animal bodies contain a very large amount of water by both weight and volume and changes in body water content have stressful consequences for most species and trigger homeostatic responses to readjust water content and restore both ionic and osmotic balance. Research on animal dehydration resistance ranges from studies of microfauna (e.g. tardigrades, brine shrimp, nematodes \& others) that can endure the loss of virtually all body water (a phenomenon called anhydrobiosis) through studies of amphibian and reptile water economy to research in exercise science that examines the effects of dehydration on cognition and physical performance by humans. However, although whole-body dehydration has been quite extensively characterized at a physiological level in vertebrate species, much less is known about the responses/adaptations of cellular metabolic pathways and gene/protein regulation in response to water loss. In climates with annual dry seasons where temperatures are high and water availability is low, amphibians and reptiles can frequently seek refuge underground to minimize dehydration, often also utilizing metabolic rate depression to enter a quiescent state of estivation (Abe, 1995; Loveridge, 1976; Seymour and Lee, 1974; Storey, 2002). Other known factors that support frog and toad survival in their underground refugia include entering estivation with a huge store of water in the bladder that is used to replenish body water as it evaporates across the skin or is lost during respiration (Balinsky et al., 1961; Groom et al., 2013) and accumulation of urea to provide osmotic resistance to body water loss. Such dehydration-tolerant animals are excellent models for studying the cellular responses of whole-body dehydration in vertebrates. 


\subsection{Whole-body dehydration in Xenopus laevis}

The African clawed frog, Xenopus laevis, is a highly aquatic species and inhabits ponds, wetlands, and lakes in sub-Saharan Africa. However, during the dry season, their habitat can dry out, and the frogs have been observed to dig into the mud to minimize dehydration - a behaviour seen in many anurans (Tinsley and Kobel, 1996). Due to their aquatic nature and unlike many frog and toad species, African clawed frogs do not have large bladders that can significantly buffer water loss due to dehydration (bladder volume may be only $\sim 1 \%$ of body mass) (Hillman, 1978a). Instead, they must turn to physiological and molecular responses to deal with dehydration and, indeed, are able to endure the loss of a substantial amount of body water (at least 30\%) as their natural habitats get progressively drier. As a result, these frogs are an ideal animal model to study the cellular responses to vertebrate whole-body dehydration, all the more so because $X$. laevis is one of the very few amphibian species with a sequenced genome (http://www.xenbase.org/genomes/static/laevis.jsp)

In order to combat dehydration stress, $X$. laevis can increase its body osmolality (Balinsky et al., 1967). For most of the year, this frog excretes nitrogenous waste in the form of ammonia into its aquatic habitat but as the frogs dehydrate, they reduce urine production and instead of excreting ammonia as waste, nitrogen is channeled into the synthesis of urea by an upregulated urea cycle. Hence, as frogs dehydrate they show reduced ammonia excretion, whereas blood plasma (15.5-fold), red cell ( $>4.34$-fold), whole blood ( $>5.57$-fold, liver (22.4-fold), and muscle (20.0-fold) urea concentrations increase (Balinsky et al., 1967, 1961; Jokumsen and Weber, 1980). By elevating this nonionic osmolyte within its body fluids, water loss across the amphibian`s water-permeable 
skin is retarded (Balinsky et al., 1961; Jørgensen, 1997). Analysis of X. laevis plasma has shown that levels of sodium, potassium, and chloride ions change as would be predicted as dehydration proceeds (Hillman, 1978a). However, plasma volume appears to decrease faster than expected, and a comparable increase in hematocrit is observed (Hillman, 1978a). Unlike other solutes that were investigated, urea levels were significantly elevated not only in plasma, but also in red blood cells and whole blood, as compared to the elevated levels expected from water loss alone (Jokumsen and Weber, 1980) and this suggested secondary effects of urea on intracellular volume regulation and/or metabolism.

By causing a decrease in plasma volume, whole-body dehydration in X. laevis increases hematocrit and blood viscosity which results in a curtailment of oxygen delivery that yields increased resting heart rate and increased difference in arterio-venous blood oxygen contents, and furthermore increases in whole-animal lactate occurring at the more extreme levels of dehydration (Hillman, 1978a).

Tissue-specific differences have been identified with respect to osmoregulatory processes in dehydrating $X$. laevis. Studies revealed that both the ventricle and gastrocnemius muscle had greater water contents than were predicted from the extent of whole body dehydration and differing from the opposite response by plasma volume, which decreases faster than predicted (Hillman, 1978b). These results suggested that $X$. laevis has homeostatic mechanisms that can prioritize water maintenance in muscle tissues over plasma volume. Indeed, when muscle water does decrease, there is a noticeable decrease in tetanic tension development which hampers mobility and muscle function (Hillman, 1978b). Hence, maintenance of intracellular water appears to be 
prioritized over water in the extracellular compartments, suggesting that tissue water homeostasis is more important than maintenance of extracellular fluid volume as amphibians dehydrate. However, studies using microspheres to track blood circulation in dehydrating $X$. laevis suggested that skeletal muscle is prioritized last among other vital organs. In all tissues analyzed, systemic blood circulation did not appear to be affected by dehydration, with the exception of skeletal muscle tissues and the brain. Dehydration was found to increase blood circulation to the brain, whereas circulation to skeletal muscle tissues decreased (Hillman and Sommerfeldt, 1981). Together, the previous studies on different tissues and organs of $X$. laevis suggest that water and blood circulation is regulated in a way that favours the intracellular protection of tissues such as the brain and vital organ systems at the expense of extracellular components such as plasma volume (Figure 1.1).

\subsection{Metabolic enzymes}

Similar to the physiological changes observed during dehydration in X. laevis, various metabolic enzymes are also differentially regulated in a tissue-specific manner. Although the total protein levels of four glycolytic enzymes were unchanged by dehydration in selected organs (brain, heart, kidney) (Wu et al., 2017), studies of the properties of purified glycolytic enzymes (hexokinase, pyruvate kinase, lactate dehydrogenase) suggested that reversible changes in enzyme function can occur in $X$. laevis in a manner that would to favor anaerobic glycolysis. This would allow the frogs to better cope with impaired oxygen circulation as dehydration progresses (Table 1.1).

An analysis of skeletal muscle hexokinase (HK), the entry point for glucose into glycolysis, showed a 3.4-fold increase in enzyme maximal activity $\left(\mathrm{V}_{\max }\right)$, as well as 
changes to the phosphorylation state of the enzyme (increased phosphoserine and decreased phosphothreonine contents) that altered HK regulation in dehydrated frogs (Childers and Storey, 2016). Although $\mathrm{HK} \mathrm{K}_{\mathrm{m}}$ for ATP was not significantly altered, there was a 2.4-fold increase in $\mathrm{K}_{\mathrm{m}}$ for glucose (Childers and Storey, 2016), suggesting that glycolysis is not necessarily inhibited during dehydration, but only proceeds when there is sufficient glucose.

At the other end of glycolysis, a characterization of skeletal muscle pyruvate kinase (PK) in X. laevis also provided evidence of a shift towards anaerobic glycolysis during dehydration. Although there were no changes in PK kinetic parameters, transcript levels of PK increased in X. laevis skeletal muscle during dehydration (Dawson et al., 2018) which could imply an increase in PK protein content and activity in this tissue. Given that blood circulation is compromised in skeletal muscle of dehydrating X. laevis in favour of prioritizing circulation to vital organs such as the brain (Hillman and Sommerfeldt, 1981), these studies mutually suggest that skeletal muscle HK and PK are regulated such that anaerobic glycolysis can sustain energy requirements during dehydration-induced oxygen curtailment.

In X. laevis liver, PK showed substantially greater regulation than the skeletal muscle enzyme. Similarly to muscle, liver PK mRNA levels increased in response to dehydration (Dawson et al., 2018). Liver PK from dehydrated X. laevis showed increased affinities for its glycolytic substrate $\left(\mathrm{K}_{\mathrm{m}}\right.$ for phosphoenolpyruvate decreased to $38 \%$ of control levels) and activators ( $\mathrm{K}_{\mathrm{a}}$ for fructose-1,6-bisphosphate decreased to $32 \%$ of control values) (Dawson et al., 2018). These changes in enzyme kinetics observed during dehydration are postulated to occur in part due to changes in protein post-translational 
modifications, since liver PK from dehydrated $X$. laevis had 25\% lower protein phosphorylation compared to the purified enzyme from control frogs. Furthermore, liver PK from dehydrated $X$. laevis was more sensitive to allosteric activators (activation in dehydration was 1.56 times higher than activation in control for fructose-1,6bisphosphate) when compared to hydrated liver PK (Dawson et al., 2018). The authors concluded that this dehydration-responsive mechanism of feed-forward activation (by the product of the phosphofructokinase reaction of glycolysis) would help coordinate the glycolysis pathway to meet energy demands, particularly when oxygen circulation is impaired, yet precursor substrates are available to utilize.

Lactate dehydrogenase (LDH) plays a role in stress tolerance whenever oxygenbased ATP generation is impaired. By converting pyruvate into lactate, the NAD ${ }^{+}$ consumed earlier in the glycolytic pathway is regenerated. Purified liver LDH of dehydrated $X$. laevis exhibited higher $\mathrm{K}_{\mathrm{m}}$ values for L-lactate $\left(1.74\right.$ fold), NAD ${ }^{+}(2.41$ fold), and pyruvate (1.78 fold) in comparison to LDH from control frogs (Katzenback et al., 2014). These results suggested a suppression of LDH activity under dehydrating conditions that is mediated by decreased affinity for substrates. Analysis of posttranslational modifications indicated that dehydration-induced changes to LDH phosphorylation, methylation, and ubiquitination may underlie the kinetic changes (Katzenback et al., 2014). However, when LDH from dehydrated frogs was assayed under physiological concentrations of urea, all three $\mathrm{K}_{\mathrm{m}}$ values returned to control levels (Katzenback et al., 2014). This demonstrates that urea accumulation in dehydrating frogs can have a positive action to maintain LDH activity and support glycolytic function during dehydration. In addition, the authors speculated that the resulting post- 
translational modifications on LDH from dehydration may play a role in facilitating the observed suppression in LDH activity when urea levels are reduced upon rehydration, however, additional studies are warranted as LDH from rehydrated $X$. laevis were not studied. Indeed, changes in protein phosphorylation can occur rapidly relative to the time it takes urea to clear upon rehydration, which is known to take days (Balinsky et al., 1961). The results suggest that $X$. laevis maintains $\mathrm{NAD}^{+}$levels by producing lactate with LDH during dehydration, but upon rehydration, the authors further speculated that LDH activity may be suppressed in order to redirect pyruvate towards the TCA cycle as oxygen circulation is restored and $\mathrm{NAD}^{+}$can be regenerated through oxidative phosphorylation. Alternatively, if LDH activity is rapidly restored upon rehydration, it may facilitate the conversion of lactate to pyruvate which can be directed towards gluconeogenesis or oxidative phosphorylation.

\subsection{Post-translational controls by protein phosphorylation}

Post-translational modifications to proteins such as by reversible phosphorylation of serine, threonine or tyrosine residues regulate the responses of many cellular processes to external signals (e.g. hormones, nutrients, abiotic stress, etc) (Table 1.1). The mitogenactivated protein kinase (MAPK) signal transduction pathways are core players in mediating responses to extracellular signals and are characterized by a 3-tiered cascade system. Each MAPK is activated upstream via phosphorylation from a MAPK kinase (MAPKK) that in turn is activated via phosphorylation by a MAPKK kinase (MAPKKK) that responds to an input signal at the cell membrane. This 3-tier system allows for a huge and rapid amplification of the input signal in activating diverse targets as well as much opportunity for cross-talk or modulation of responses (Cowan and Storey, 2003). One 
established MAPK pathway that has been characterized in multiple tissues of $X$. laevis in response to dehydration is the extracellular signal-regulated kinase (ERK) pathway. At the upstream end of the ERK pathway, phosphorylated active c-Raf (Ser338) (a MAPKKK) was found to increase in skeletal muscle, lung, and skin in response to dehydration exposure (but not in kidney and heart) (Malik and Storey, 2009a). Similarly, the protein levels of MEKK, another MAPKKK also increased under dehydration in skeletal muscle, lung, kidney, and liver (Malik and Storey, 2009a). However, total MEKK protein levels decreased in the heart and skin of dehydrating X. laevis (Malik and Storey, 2009a). These results suggest that dehydration in X. laevis regulate the ERK signaling pathway in a tissue specific manner.

The upstream c-Raf and MEKK proteins activate the MEK1/2 proteins by phosphorylating them. In dehydrating $X$. laevis, total protein and phosphorylated protein analyses of MEK1/2 suggested a clear activation in this target in all 6 tissues tested (skeletal muscle, lung, kidney, heart, skin and liver) (Malik and Storey, 2009a). Furthermore, there was a strong correlation between c-Raf activation and MEK1/2 phosphorylation in skeletal muscle, lung, and skin tissues of dehydrated X. laevis. A trend was also observed between MEKK activation and phosphorylation of MEK1/2 in the kidney, lung, and liver (Malik and Storey, 2009a). Despite an increase in MEK1/2 in heart of dehydrated $X$. laevis, a there was no correlation with respective c-Raf and MEKK data which either showed no change, or a dehydration-induced decrease in total protein levels (Malik and Storey, 2009a). This work on dehydrating X. laevis demonstrated that the MAPK pathway is active in response to dehydration, but the response is complex and 
tissue specific, likely involving the interactions of additional kinases that have yet to be explored in the context of $X$. laevis whole-body dehydration.

The role of activated MEKK is to activate ERK by phosphorylation. Various stages of dehydration resulted in increased levels of activated (phosphorylated) ERK2 in X. laevis skeletal muscle, lung, kidney, heart and liver (but not in skin) (Malik and Storey, 2009a). This again demonstrates the general response of MAPKs in X. laevis to dehydration, and emphasizes their tissue-specific nature. One of the major roles of MAPKs is to regulate gene transcription by phosphorylating transcription factor substrates. The signal transducer and activators of transcription (STAT) family of transcription factors is one group that is activated by MAPK phosphorylation, which causes these proteins to form homo- or heterodimers that facilitate their transportation from the cytoplasm into the nucleus (Mitchell and John, 2005). The STAT3 member is a substrate of ERK2 (Chung et al., 1997; Krishna and Narang, 2008), and phosphorylation of both proteins increased with dehydration in X. laevis skeletal muscle, lung, and kidney (Malik and Storey, 2009a). Since this transcription factor is well known to facilitate the expression of genes encoding anti-apoptotic Bcl2 protein family members such as Bcl-2 and Mcl-1 (Bhattacharya et al., 2005), ERK-mediated activation of the anti-apoptosis pathway via STAT3 phosphorylation may be another mechanism by which $X$. laevis copes with dehydration on a cellular level.

\subsection{Upregulation of antioxidants by transcription factors}

Other transcription factors, such as forkhead box class $\mathrm{O}$ type 1 (FoxO1), also play important roles in the $X$. laevis dehydration response. Similar to STAT3, the activity of FoxO1 is regulated by post-translational modifications, and is differentially 
phosphorylated in the liver of dehydrating X. laevis (Malik and Storey, 2011). During dehydration, relative levels of inhibitory phosphorylation of liver FoxO1 (Ser245) were reduced for both the total and nucleus-localized protein, and there was a 1.8-fold increase in FoxO1 localization to the nucleus (Malik and Storey, 2011). Furthermore, a relative increase in liver FoxO1 DNA binding occurred. All of these factors indicate that FoxO1 is activated during dehydration in $X$. laevis liver. The FoxO1 transcription factor has a role in upregulating antioxidant defenses by promoting the expression of genes encoding enzymes such as manganese-dependent superoxide dismutase (MnSOD) and catalase (Malik and Storey, 2011). Indeed, both mRNA and protein levels of MnSOD and catalase were significantly increased in the liver during dehydration (Malik and Storey, 2011). Given that oxygen levels can become reduced due to ischemic-like conditions during dehydration, as evidenced by an increased difference in arterio-venous oxygen concentrations (Hillman, 1978a), X. laevis may be undergoing "preparation for oxidative stress" (POS) (Moreira et al., 2017), a phenomenon that is often part of stress-responsive adaptation whereby an organism reacts both to an environmental stress (e.g. oxygen limitation) and also prepares at the same time for a reversal of the stress (e.g. a rapid increase in reactive oxygen species (ROS) when oxygen is reintroduced). The activation of FoxO1-mediated antioxidant enzymes was tissue specific in $X$. laevis, since comparable responses did not occur in skeletal muscle, with the exception of an increase in catalase protein levels (Malik and Storey, 2011). Although both tissues are sensitive to low oxygen during dehydration, a more prominent cytoprotective response in the liver may signify the importance of this organ during dehydration and lead one to hypothesize that it undergoes significant metabolic changes such as modifications in oxygen- 
dependent metabolism and ureagenesis. The POS phenomenon has been documented in a wide range of animals across phylogeny including among cnidarians (a basal organism of the eumetazoa) which suggests that POS may have been present as far back as 700 million years ago to aid animals in adapting to oxygen/oxidative stress in their environments such as under hypoxic, anoxic, and freezing conditions (Moreira et al., 2017). An increase in antioxidant abundance may help these animals endure the sudden influx of oxygen in their return to normoxia, thawing, and in the case of X. laevis, rehydration.

Deployment of antioxidants is also facilitated by the transcription factor Nuclear factor-like 2 (Nrf2), which is activated in multiple X. laevis tissues during dehydration. Similar to FoxO1, Nrf2 also promotes the expression of antioxidants such as MnSOD and catalase (Zhu et al., 2005). In dehydrating X. laevis, Nrf2 mRNA levels were elevated in liver, but were unchanged in skeletal muscle (Malik and Storey, 2009b). In contrast, nuclear localization of muscle Nrf2 increased in dehydration, whereas there was no effect on localization in the liver (Malik and Storey, 2009b). These differences in transcription factor regulation demonstrate that control of these pathways is complex and tissuespecific. However, the changes suggest an overall pattern of activation for these antioxidant pathways. This applies over a broad range of tissues since total Nrf2 protein levels were also elevated in lung, heart, and skin tissues, in addition to the liver where the increase was as high as 4-fold (Malik and Storey, 2009b). Among other responses (Table 1), antioxidant activation in X. laevis likely plays an important role in enduring bouts of dehydration and rehydration. 


\subsection{Advances in the $X$. laevis genome}

There are many anurans that match or exceed the level of dehydration tolerance exhibited by X. laevis. As the field moves forward in elucidating the molecular mechanisms that aid dehydration resistance among vertebrate species, an ideal vertebrate model must be identified. Resulting from decades of research as a model for embryonic developmental and the widespread use of Xenopus eggs as a convenient system for transgenic research, X. laevis (a tetraploid species) became the second amphibian species to be genome-sequenced shortly after its relative, $X$. tropicalis (a diploid). Because of this, X. laevis has distinct advantages as model organism in the study of molecular responses to dehydration. The availability of genomic and proteomic data has accelerated research on animal stress response and adaptation (Biggar and Storey, 2014; Storey and Wu, 2013). The large databases available for Xenopus species now allow extensive bioinformatic data mining, which has made possible studies such as the one that identified novel dehydration-responsive genes in X. laevis such as drp10. The drp10 gene encodes a structural homolog of a freeze-responsive protein called FR10 that occurs in freeze-tolerant wood frogs (Rana sylvatica) (Biggar et al., 2015). Interestingly, drp10 transcript levels increased in X. laevis during dehydration in both liver and kidney and this implicated both the DRP10 and FR10 proteins in issues of cell water regulation in widely diverse amphibian species (southern Africa versus northern Canada). Another recent and significant advancement in sequencing and bioinformatics has resulted in the detailed annotation and characterization of the $X$. laevis allotetraploid genome. By partitioning the whole allotetraploid genome into two homologous subgenomes, researchers were able to estimate that the two diploid progenitor species diverged $\sim 34$ million years ago, and combined to form an allotetraploid 17-18 million years ago 
(Session et al., 2016). The two subgenomes were found to have evolved asymmetrically, where one genome is most representative of the ancestral state, whereas the other demonstrated more gene loss, deletion, rearrangement, and reduced gene expression (Session et al., 2016). In all, advances in X. laevis genome research have allowed for scientists to more smoothly navigate the complexities of an allotetraploid animal model's genome, making this model organism a top candidate in which biologists can study the molecular mechanisms of vertebrate whole-body dehydration. 


\section{Hypothesis}

The molecular responses to whole-body dehydration in the African clawed frog (Xenopus laevis) involve the regulation of genes and proteins that control posttranslational acetylation, cytoprotective responses, and metabolic pathways in a tissue-specific manner.

My hypothesis builds on current knowledge of whole-body $X$. laevis dehydration by characterizing highly conserved cellular signaling pathways have been thoroughly elucidated in other experimental models such as cell lines and in vivo rodent models described in the following objectives:

\section{Objective 1: Post-translational Regulation during Whole-Body Dehydration by} NAD $^{+}$-Dependent Sirtuin Protein Deacetylases

Control of proteins by reversible post-translational acetylation has the potential of regulating processes that may be tightly controlled during whole-body dehydration in $X$. laevis, which have roles in metabolism and cytoprotection. In order to better understand the potential roles of Sirtuin (Sirt) deacetylases during dehydration, studies must be performed to identify which of the highly conserved Sirt family members are modulated, to reveal in which tissues these regulators have a role, and whether or not their targets exhibit decreased acetylation during dehydration.

\section{Specific Hypothesis 1: Individual sirt genes are regulated at the mRNA gene transcript} level, general Sirtuin deacetylase activity is increased, and known targets of Sirt proteins are deacetylated in dehydrated $X$. laevis tissues in a manner that supports increased ureagenesis, antioxidant upregulation, and decreased oxygen-dependent metabolism. 
In Chapter 2, this hypothesis is tested by profiling the expression of all 7 known sirt genes in 6 X. laevis tissues. Identification of sirt genes that are upregulated in response to dehydration underscores their importance to water loss, as the animal warrants the expenditure of energy to undergo the transcription of these genes. As each of the 7 members of the Sirt family have unique roles that span the regulation of antioxidants, metabolism, and angiogenesis, the results from this chapter not only show that sirt gene expression is modulated during dehydration, but also will highlight the processes which may be modulated by Sirt proteins during dehydration.

Although increases in mRNA levels shows that the cells are willing to spend precious energy to facilitate the transcription of these genes, they do not provide any evidence as to whether these gene transcripts are translated into protein, or whether there is any increase or decrease in deacetylase activity. Chapter $\mathbf{2}$ also addresses this hypothesis by measuring global Sirt deacetylase activity of multiple tissues from animals under control and dehydrated conditions. Furthermore, relative levels of highly conserved protein targets that Sirt deacetylases are known to modulate are assessed to provide further evidence to support that the activities of these deacetylases are changing during dehydration.

\section{Objective 2: Sirtuin and PGC-1 $\alpha$ regulated antioxidant pathways}

The interactions between specific Sirtuin proteins and transcriptional regulators have been thoroughly characterized in other experimental models (such as cell lines and in vivo rodent models). These studies utilize a wide range of loss-of-function/gain-offunction strategies to elucidate the specific roles of select Sirt proteins and their targets. 
Some Sirt proteins have been shown to play direct roles in upregulating antioxidants through their interactions with other transcriptional regulators such as Peroxisome proliferator-activated receptor gamma coactivator 1-alpha (PGC-1 $\alpha$ ) and the FoxO transcription factors - key targets that are investigated to reveal the state of antioxidant cellular signaling during dehydration.

\section{Specific Hypothesis 2: The response to whole-body dehydration by $X$. laevis implicates} the activation of the Sirt/PGC-1 $\alpha /$ FoxO signaling pathway, upregulated antioxidants, and sustained antioxidant capacity.

As previously stated, this hypothesis is addressed in Chapter 2 as all sirt mRNA targets are assessed in multiple tissues, including those that, according to the literature, contribute to the maintenance or upregulation of antioxidants. Furthermore, global Sirt deacetylase activity will contribute to addressing this hypothesis by showing whether or not increases in Sirt deacetylase expression is accompanied by increases in deacetylase activity. Lastly, measurements of acetylated antioxidant enzymes are also done in Chapter 2, further exploring whether or not the Sirt response may contribute to antioxidant upregulation.

In Chapter 3, the hypothesis is addressed by measuring protein levels of Sirt proteins that are known to regulate antioxidants (Sirt1, Sirt2, Sirt3 and Sirt6). Measurements are done in the lung, a "first responder" tissue that is susceptible to water loss during dehydration due to continued breathing. Results from the lung are compared with those from brain, where increased blood circulation is observed during dehydration (Hillman and Sommerfeldt, 1981) which is assumed to be a neuroprotective effect. One 
thoroughly characterized interaction in the literature is that between Sirt proteins, the transcriptional coactivator PGC-1 $\alpha$, and FoxO transcription factors and their combined role in upregulating antioxidants. In order to address the hypothesis, these targets are characterized on the mRNA, protein, and posttranslational levels (posttranslational modifications).

This hypothesis is also addressed in Chapter 3 with the measurements of targets downstream of Sirt/PGC-1 $\alpha /$ FoxO, including catalase, heme oxygenase-1 (HO-1), SOD1, and SOD2 at the mRNA and protein levels. Lastly, measurements of $X$. laevis cell lysates were performed to reveal how increases in cellular pathways that upregulate antioxidants may contribute to the maintenance of antioxidant capacity during dehydration.

\section{Objective 3: Cellular signaling pathways controlling metabolism and angiogenesis.}

Evidence from previous studies has suggested that whole-body dehydration, in the context of $X$. laevis, impairs the circulatory system and hampers oxygen transport. The frog attempts to compensate for this by elevating its heartrate, rather than decreasing it as other animals do when they decrease their metabolic rate. Over the course of dehydration, arterio-venous differences in oxygen levels increase, which suggests the presence of challenges in sustaining levels oxygenated blood as compared to hydrated animals. Furthermore, whole-animal lactate is observed to increase at the higher stages of dehydration. Together, this leads to the hypothesis that cellular signaling pathways are remodelled so that ureagenesis and angiogenesis are supported while oxygen-dependent metabolism is suppressed in favour of anaerobic glycolysis. 


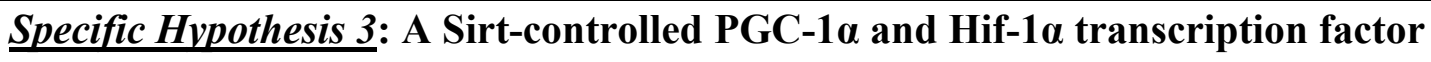
signaling network is activated during whole-body dehydration in $X$. laevis that results in the differential expression of genes which would be characteristic of increased angiogenesis, increased ureagenesis, and suppressed oxygen-dependent metabolism.

In Chapter 2, this hypothesis was addressed by profiling the expression patterns of sirt genes which are known to have regulatory roles in fatty acid oxidation, ureagenesis, and angiogenesis, either directly or through the interaction with other targets such as the transcriptional coactivator PGC-1 $\alpha$.

Chapter 4 focuses on the metabolic roles if PGC-1 $\alpha$ in the liver, skeletal muscle, and heart. Sirt-activated PGC-1 $\alpha$ has the potential to increase cytoprotective responses such as the upregulation of antioxidants, but also the induction of genes that translate into proteins that facilitate fatty acid oxidation - a process that is not expected to occur during X. laevis dehydration. Additionally, Sirt-activated PGC-1 $\alpha$ is also expected to occur during dehydration as it is known to activate ureagenesis by inducing the mRNA and protein expression of other Sirts (Sirt3 and Sirt5), which deacetylate and increase the activity of urea cycle enzymes. This chapter addresses the hypothesis by profiling PGC$1 \alpha$ and its upstream regulator, myocyte enhancer factor-2 (MEF2), at the mRNA and protein levels, in addition to analysis of related downstream mRNA targets that are required for fatty acid oxidation and ureagenesis.

The Hypoxia-inducible factor-1 alpha (Hif-1 $\alpha)$ is a transcription factor that is directly targeted by Sirts, leading to its deacetylation, stabilization, and activation. Unlike 
PGC-1 $\alpha$, Hif- $1 \alpha$, which is also activated by Sirt proteins, is known to suppress the expression of fatty acid oxidation genes, while promoting the expression of genes required for glucose metabolism and angiogenesis. Chapter $\mathbf{4}$ addresses the hypothesis by characterizing Hif- $1 \alpha$ at both mRNA and protein levels, in addition to the profiling of its downstream mRNA targets that are required for the translation of proteins involved in glucose metabolism and angiogenesis. 


\subsection{References}

Abe, A.S., 1995. Estivation in South American amphibians and reptiles. Brazilian J. Med. Biol. Res.

Balinsky, J.B., Choritz, E.L., Coe, C.G., van der Schans, G.S., 1967. Amino acid metabolism and urea synthesis in naturally aestivating Xenopus laevis. Comp. Biochem. Physiol. 22, 59-68.

Balinsky, J.B., Cragg, M.M., Baldwin, E., 1961. The adaptation of amphibian waste nitrogen excretion to dehydration. Comp. Biochem. Physiol. 3, 236-244. doi:10.1016/0010-406X(61)90009-3

Bhattacharya, S., Ray, R.M., Johnson, L.R., 2005. STAT3-mediated transcription of Bcl2, Mcl-1 and c-IAP2 prevents apoptosis in polyamine-depleted cells. Biochem. J. 392, 335-344. doi:10.1042/BJ20050465

Biggar, K.K., Biggar, Y., Storey, K.B., 2015. Identification of a novel dehydration responsive gene, drp10, from the African clawed frog, Xenopus laevis. J. Exp. Zool. A. Ecol. Genet. Physiol. 323, 375-81. doi:10.1002/jez.1930

Biggar, K.K., Storey, K.B., 2014. New Approaches to Comparative and Animal Stress Biology Research in the Post-genomic Era: A Contextual Overview. Comput. Struct. Biotechnol. J. doi:10.1016/j.csbj.2014.09.006

Childers, C.L., Storey, K.B., 2016. Post-translational Regulation of Hexokinase Function and Protein Stability in the Aestivating Frog Xenopus laevis. Protein J. 35, 61-71. doi:10.1007/s10930-016-9647-0

Chung, J., Uchida, E., Grammer, T.C., Blenis, J., 1997. STAT3 serine phosphorylation by ERK-dependent and -independent pathways negatively modulates its tyrosine phosphorylation. Mol Cell Biol 17, 6508-6516. doi:10.1128/MCB.17.11.6508

Cowan, K.J., Storey, K.B., 2003. Mitogen-activated protein kinases: new signaling pathways functioning in cellular responses to environmental stress. J. Exp. Biol. 206, 1107-1115. doi:10.1242/jeb.00220

Dawson, N.J., Biggar, Y., Malik, A.I., Storey, K.B., 2018. Increased transcript levels and kinetic function of pyruvate kinase during severe dehydration in aestivating African clawed frogs, Xenopus laevis. Comp. Biochem. Physiol. Part B Biochem. Mol. Biol. doi:10.1016/J.CBPB.2018.01.003

Groom, D.J.E., Kuchel, L., Richards, J.G., 2013. Metabolic responses of the South American ornate horned frog (Ceratophrys ornata) to estivation. Comp. Biochem. Physiol. Part B Biochem. Mol. Biol. 164, 2-9. doi:10.1016/j.cbpb.2012.08.001

Hillman, S.S., 1978a. The roles of oxygen delivery and electrolyte levels in the dehydrational death of Xenopus laevis. J. Comp. Physiol. B 128, 169-175. doi:10.1007/BF00689481 
Hillman, S.S., 1978b. Some effects of dehydration on internal distributions of water and solutes in Xenopus laevis. Comp. Biochem. Physiol. -- Part A Physiol. 61, 303-307. doi:10.1016/0300-9629(78)90113-5

Hillman, S.S., Sommerfeldt, R.W., 1981. Microsphere studies of amphibian systemic blood flow redistribution during dehydration, hypovolemia, and salt load. J. Exp. Zool. 218, 305-308. doi:10.1002/jez.1402180223

Jokumsen, A., Weber, R.E., 1980. Haemoglobin-Oxygen Binding Properties in the Blood of Xenopus Laevis, with Special Reference to the Influences of Aestivation and of Temperature and Salinity Acclimation. J. Exp. Biol. 86, 19-37.

Jørgensen, C.B., 1997. Urea and amphibian water economy. Comp. Biochem. Physiol. A. Physiol. 117, 161-70.

Katzenback, B.A., Dawson, N.J., Storey, K.B., 2014. Purification and characterization of a urea sensitive lactate dehydrogenase from the liver of the African clawed frog, Xenopus laevis. J. Comp. Physiol. B 184, 601-611. doi:10.1007/s00360-014-0824-1

Krishna, M., Narang, H., 2008. The complexity of mitogen-activated protein kinases (MAPKs) made simple. Cell. Mol. Life Sci. doi:10.1007/s00018-008-8170-7

Loveridge, J.P., 1976. Strategies of Water Conservation in Southern African Frogs. Zool. Africana 11, 319-333. doi:10.1080/00445096.1976.11447538

Malik, A.I., Storey, K.B., 2011. Transcriptional regulation of antioxidant enzymes by FoxO1 under dehydration stress. Gene 485, 114-119.

doi:10.1016/j.gene.2011.06.014

Malik, A.I., Storey, K.B., 2009a. Activation of extracellular signal-regulated kinases during dehydration in the African clawed frog, Xenopus laevis. J. Exp. Biol. 212.

Malik, A.I., Storey, K.B., 2009b. Activation of antioxidant defense during dehydration stress in the African clawed frog. Gene 442, 99-107.

doi:10.1016/j.gene.2009.04.007

Mitchell, T.J., John, S., 2005. Signal transducer and activator of transcription (STAT) signalling and T-cell lymphomas. Immunology. doi:10.1111/j.1365-

2567.2005.02091.x

Moreira, D.C., Oliveira, M.F., Liz-Guimarães, L., Diniz-Rojas, N., Campos, É.G., Hermes-Lima, M., 2017. Current trends and research challenges regarding "preparation for oxidative stress." Front. Physiol. 8, 702. doi:10.3389/fphys.2017.00702

Session, A.M., Uno, Y., Kwon, T., Chapman, J.A., Toyoda, A., Takahashi, S., Fukui, A., Hikosaka, A., Suzuki, A., Kondo, M., van Heeringen, S.J., Quigley, I., Heinz, S., Ogino, H., Ochi, H., Hellsten, U., Lyons, J.B., Simakov, O., Putnam, N., Stites, J., Kuroki, Y., Tanaka, T., Michiue, T., Watanabe, M., Bogdanovic, O., Lister, R., Georgiou, G., Paranjpe, S.S., van Kruijsbergen, I., Shu, S., Carlson, J., Kinoshita, T., 
Ohta, Y., Mawaribuchi, S., Jenkins, J., Grimwood, J., Schmutz, J., Mitros, T., Mozaffari, S. V., Suzuki, Y., Haramoto, Y., Yamamoto, T.S., Takagi, C., Heald, R., Miller, K., Haudenschild, C., Kitzman, J., Nakayama, T., Izutsu, Y., Robert, J., Fortriede, J., Burns, K., Lotay, V., Karimi, K., Yasuoka, Y., Dichmann, D.S., Flajnik, M.F., Houston, D.W., Shendure, J., DuPasquier, L., Vize, P.D., Zorn, A.M., Ito, M., Marcotte, E.M., Wallingford, J.B., Ito, Y., Asashima, M., Ueno, N., Matsuda, Y., Veenstra, G.J.C., Fujiyama, A., Harland, R.M., Taira, M., Rokhsar, D.S., 2016. Genome evolution in the allotetraploid frog Xenopus laevis. Nature 538, 336-343. doi:10.1038/nature19840

Seymour, R.S., Lee, A.K., 1974. Physiological adaptations of anuran amphibians to aridity: Australian prospects. Aust. Zool. 18, 53-65.

Storey, K.B., 2002. Life in the slow lane: Molecular mechanisms of estivation. Comp. Biochem. Physiol. - A Mol. Integr. Physiol. 133, 733-754. doi:10.1016/S10956433(02)00206-4

Storey, K.B., Wu, C.-W., 2013. Stress response and adaptation: a new molecular toolkit for the 21st century. Comp. Biochem. Physiol. A. Mol. Integr. Physiol. 165, 417-28. doi:10.1016/j.cbpa.2013.01.019

Tinsley, R.C., Kobel, H.R., 1996. The biology of Xenopus. Clarendon Press.

Wu, C.-W., Tessier, S.N., Storey, K.B., 2017. Regulation of the insulin-Akt signaling pathway and glycolysis during dehydration stress in the African clawed frog Xenopus laevis. Biochem. Cell Biol. 95, 663-671. doi:10.1139/bcb-2017-0117

Zhu, H., Itoh, K., Yamamoto, M., Zweier, J.L., Li, Y., 2005. Role of Nrf2 signaling in regulation of antioxidants and phase 2 enzymes in cardiac fibroblasts: Protection against reactive oxygen and nitrogen species-induced cell injury. FEBS Lett. 579, 3029-3036. doi:10.1016/j.febslet.2005.04.058 


\section{Region-specific responses to whole-body dehydration in X. laevis}

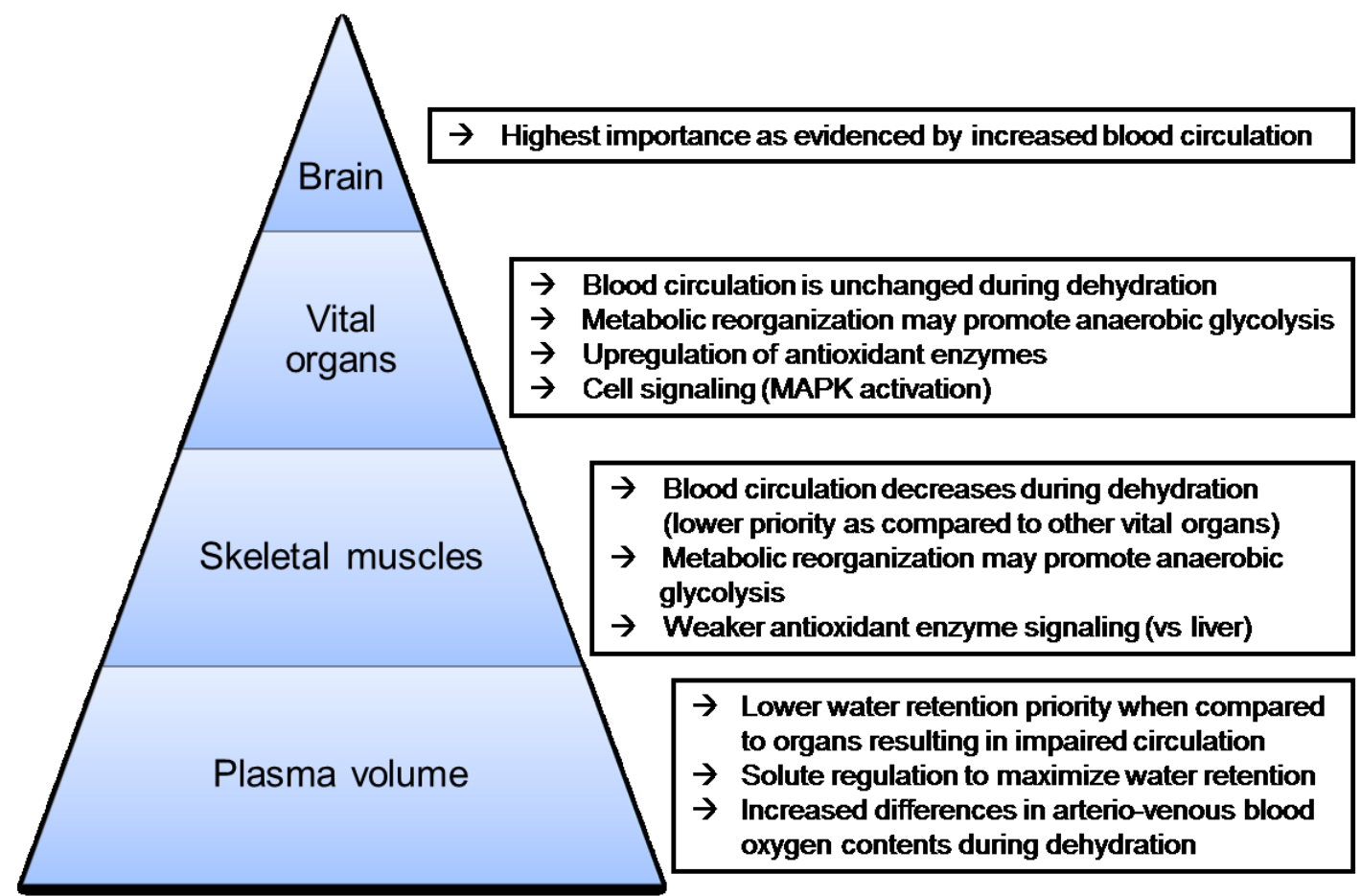

Figure 1.1. Whole-body dehydration in $X$. laevis results in a diverse set of responses that help the animal cope with impaired oxygen transport due to reduced plasma volume and increased hematocrit. The brain maintains the highest priority for blood circulation, next to other vital organs, and with skeletal muscle ranked last. Evidence suggests that metabolism shifts towards anaerobic glycolysis during dehydration as whole animal lactate increases at the higher stages of dehydration. 
Table 1.1. A summary of physiological and molecular responses that occur with wholebody dehydration in $X$. laevis, and their implications.

\begin{tabular}{|c|c|}
\hline Responses & Implications \\
\hline $\begin{array}{l}\text { Decrease in } \\
\text { plasma volume, } \\
\text { increase in } \\
\text { hematocrit }\end{array}$ & $\begin{array}{l}>\text { Impairment in oxygen delivery } \\
\text { Elevated resting heart rate and arterio-venous differences in } \\
\text { oxygen levels } \\
>\text { Decreased blood circulation to skeletal muscles }\end{array}$ \\
\hline $\begin{array}{c}\text { Elevated urea } \\
\text { levels }\end{array}$ & $>$ Increased osmolyte concentrations facilitate water retention \\
\hline $\begin{array}{l}\text { Metabolic } \\
\text { enzymes shift to } \\
\text { anaerobic } \\
\text { glycolysis }\end{array}$ & 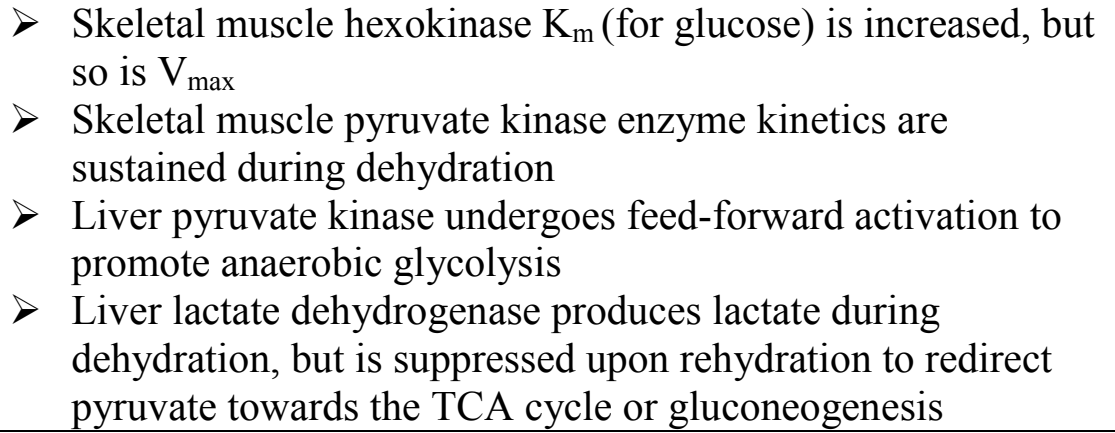 \\
\hline $\begin{array}{l}\text { Reversible protein } \\
\text { phosphorylation }\end{array}$ & $\begin{array}{l}\text { Protein phosphorylation cascades composed of MAPKs are } \\
\text { regulated in a tissue-specific manner during dehydration, with } \\
\text { signaling activated overall } \\
\text { MAPK stimulation can activate diverse responses by } \\
\text { transcription factor-mediated gene transcription }\end{array}$ \\
\hline $\begin{array}{l}\text { Antioxidant } \\
\text { signaling }\end{array}$ & $\begin{array}{l}\text { FoxO1 and Nrf2 transcription factor activation promote an } \\
\text { antioxidant defense response } \\
\text { Antioxidant enzymes such as catalase and superoxide } \\
\text { dismutase prepare the animal for oxidative stress encountered } \\
\text { during bouts of dehydration and rehydration }\end{array}$ \\
\hline
\end{tabular}




\section{CHAPTER 2}

Regulation of Sirtuins and Protein Acetylation as a Molecular Response to Dehydration 


\subsection{Introduction}

\section{$\underline{\text { 2.1.1 Post-translational modifications in } X \text {. laevis dehydration }}$}

Post-translational modifications of proteins are a major part of the response to dehydration in $X$. laevis. Reversible protein phosphorylation is a well-studied modification that is widely used by animals to adapt to arid environments (Storey and Storey, 2012). These versatile modifications control cellular processes ranging from mitogen activated protein kinase (MAPK) signaling cascades, to individual proteins such as transcription factors or metabolic enzymes (Storey and Storey, 2012). An example of a MAPK signaling cascade is the extracellular signal-regulated kinases (ERK) pathway, which is activated during $x$ whole-body dehydration in $X$. laevis and can provide prosurvival signaling by communicating with other transcription factors (Malik and Storey, 2009). One transcription factor that is regulated by post-translational phosphorylation is the forkhead box class $\mathrm{O}$ type 1 (FoxO1) protein. The transcriptional activity of FoxO1 is repressed with inhibitory phosphorylation, and during $X$. laevis dehydration, relative levels of inhibitory phosphorylation are reduced in liver (Malik and Storey, 2011). The FoxO1 transcription factor has a role in upregulating antioxidant defenses by promoting the expression of genes encoding enzymes such as Mn-dependent superoxide dismutase (SOD2) and catalase. In the same study, it was also found that mRNA and protein levels of SOD2 and catalase increased in the liver during dehydration (Malik and Storey, 2011). These select examples demonstrate how post-translational modifications such as protein phosphorylation are important in the molecular responses to dehydration in X. laevis. 


\subsubsection{Roles of protein acetylation}

Protein acetylation is another type of post-translational modification that is increasingly understood to be an important regulator of protein activity. This modification involves the covalent addition of an acetyl moiety $\left(-\mathrm{COCH}_{3}\right)$ to lysine residues of proteins by lysine acetyltransferases. Protein acetylation is commonly known for its role in the regulation of histones and nuclear transcription regulators. However, it is now known that lysine acetylation is a prevalent modification to many enzymes that catalyze metabolic processes. Virtually every enzyme in glycolysis, gluconeogenesis, the TCA cycle, the urea cycle, fatty acid metabolism, and glycogen metabolism was found to be acetylated in human liver (Zhao et al., 2010). For example, it was shown that acetylation activates a number of enzymes involved in fatty acid oxidation, but inhibited others, such as argininosuccinate lyase of the urea cycle (Zhao et al., 2010). Given that metabolic enzymes purified from tissues of dehydrated $X$. laevis show relative changes in kinetic properties and phosphorylation states (Childers and Storey, 2016; Dawson et al., 2018; Katzenback et al., 2014), it can be hypothesized that similar regulation by protein acetylation could also play a significant role in enzymatic control during dehydration.

\subsubsection{Protein deacetylases}

In the same manner that protein phosphorylation is a reversible process, so is protein acetylation; the action of acetyltransferases in adding acetyl groups to protein residues is reversed by deacetylases that remove this modification. Four classes of deacetylases are known. Classes I, II and IV encompass the histone deacetylases (HDACs) 1 to 11, and are all zinc-dependent enzymes (Vannini et al., 2004). Class III 
deacetylases, known as sirtuins, are composed of 7 members in vertebrates, (SIRT 1-7) and are homologous to the silent information regulator (or Sir2) gene in yeast (Pantazi et al., 2013). Six of these sirtuins -7) have $\mathrm{NAD}^{+}$-dependent deacetylase activity (see below; Figure 2.1), with the exception of SIRT4, that only shows NAD ${ }^{+}$-dependent ADPribosyltransferase activity (Pantazi et al., 2013).

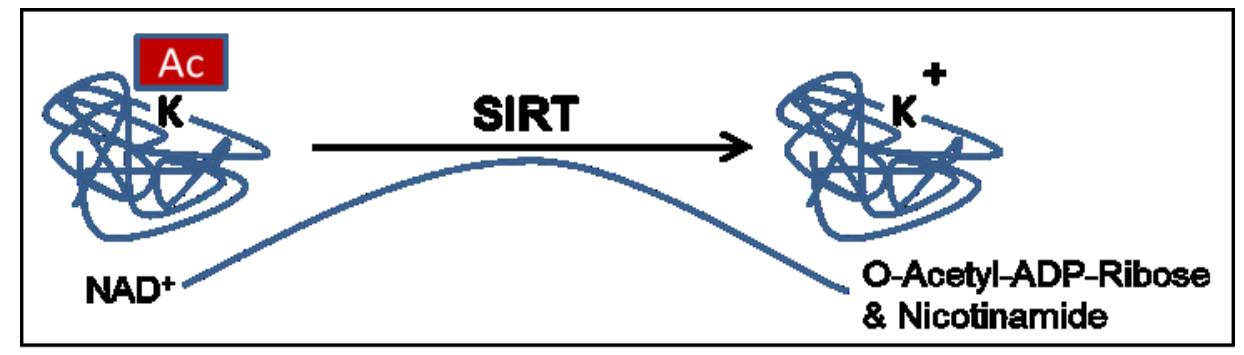

It was initially believed that these $\mathrm{NAD}^{+}$-dependent enzymes are tightly localized in the cell: SIRT1, SIRT6, and SIRT7 were generally found in the nucleus, SIRT2 was in the cytosol, and SIRT3-5 was localized in the mitochondria (Pantazi et al., 2013). However, SIRT1 has now been found to function in the cytosol, and SIRT2 has also been found in the nucleus (Morris et al., 2011) and mitochondria (Liu et al., 2017). In addition to metabolic controls (e.g. ureagenesis and fatty acid oxidation), sirtuins are known to be important in the ischemia/reperfusion response, which implicates them not only metabolic reprogramming, but also in antioxidant and cytoprotective responses (Pantazi et al., 2013). Due to the challenges in oxygen circulation that accompany dehydration, regulation of protein acetylation by Sirts is a theme that warrants exploration in the context of $X$. laevis whole-body dehydration. 


\subsubsection{Chapter objectives}

In this chapter, the expression of all 7 sirtuins (Sirts) was characterized on an mRNA level in 6 tissues (liver, skeletal muscle, brain, heart, lung, and kidney) of $X$. laevis. To do this, total RNA was extracted from tissues of control and dehydrated animals, followed by cDNA synthesis and then RT-qPCR to perform relative gene quantification. The results reveal that specific Sirts that are important to the dehydration response, as evidenced by their enhanced transcription in dehydrated animals. Global Sirt deacetylase activity assay was also measured to determine whether or not gene up- or down- regulation during dehydration led to corresponding increases or decreases in tissue Sirt $\mathrm{NAD}^{+}$-dependent deacetylase activity. By correlating known functions of the different Sirt isozymes from the literature with the particular sirt gene transcripts that were expressed in response to dehydration, and the relative changes in deacetylase activities in the different tissues, inferences were then made as to the putative roles of deacetylase activity in the $X$. laevis dehydration response. Lastly, immunoblotting of protein extracts from the 6 tissues of control versus dehydrated $X$. laevis was carried out to quantify the relative levels of lysine-acetylated superoxide dismutase 2 [Ac-SOD (K70)], a known substrate of Sirts, in order to provide a closer confirmation of deacetylase action in dehydrating tissues. 


\subsection{Materials and Methods}

\section{$\underline{2.2 .1 \text { Animals }}$}

Adult male African clawed frogs (X. laevis) were purchased from a colony at the University of Toronto and upon delivery the frogs were acclimated in tanks of dechlorinated water at $22 \pm 1^{\circ} \mathrm{C}$ for 3 weeks prior to the start of experiments. Frogs were fed 3-4 pellets of CU Adult Frog diet (PMI Nutrition International) per frog three times a week and water was changed the day after each feeding. Frogs were then randomly divided into experimental groups of control, medium dehydration, and high dehydration conditions and were not fed again. For the dehydration experiments, the frogs were individually weighed and placed into dry containers at $22 \pm 1{ }^{\circ} \mathrm{C}$ where water was lost through evaporation over time. Animals were weighed at approximately $12 \mathrm{~h}$ intervals to determine body water loss due to evaporation. To quantify the extent of dehydration, the percentage of total body water lost was calculated as follows:

$$
\% \text { water lost }=\frac{\left(\mathrm{m}_{\mathrm{i}}-\mathrm{m}_{\mathrm{d}}\right)}{\left(\mathrm{m}_{\mathrm{i}}-\mathrm{BWC} \mathrm{C}_{\mathrm{i}}\right)} \times 100 \%
$$

where $\mathrm{m}_{\mathrm{i}}, \mathrm{m}_{\mathrm{d}}$, and $\mathrm{BWC}_{\mathrm{i}}$ are initial mass, dehydrated mass, and initial body water content of the frogs, respectively. For the purpose of this study, $\mathrm{BWC}_{\mathrm{i}}$ of $X$. laevis frogs was considered to be $0.74 \pm 0.02 \mathrm{~g} \mathrm{H}_{2} \mathrm{O}$ per g body mass as previously reported (Malik and Storey, 2009). Animals in the medium and high dehydration groups were sampled when mean total body water loss reached $\sim 16 \%$ and $\sim 30 \%$, respectively. The final mean percentages for total body water loss were $16.43 \pm 0.33 \%$ SEM for the medium dehydration group, and $31.18 \pm 0.83 \%$ SEM for high dehydration group. All frogs were euthanized swiftly by pithing, and tissues were rapidly dissected, frozen in liquid nitrogen, and stored at $-80^{\circ} \mathrm{C}$ until use. At least 4 independent biological replicates 
(tissues from different animals) of liver, skeletal muscle, brain, heart, lung, and kidney were analyzed.

Animals were cared for in accordance with the guidelines of the Canadian Council on Animal Care and all experimental procedures had the prior approval of the Carleton University Animal Care Committee.

\subsubsection{RNA Extraction}

Total RNA extraction was performed using TRIzol reagent (Invitrogen, Carlsbad, CA). Frozen tissue samples ( $\sim 50 \mathrm{mg})$ were homogenized in $1 \mathrm{~mL}$ of TRIzol reagent, followed by the addition of $200 \mu \mathrm{L}$ of chloroform. Samples were vigorously shaken by hand and allowed to incubate at room temperature for $5 \mathrm{~min}$ before centrifugation at $10,000 \times \mathrm{g}$ for $15 \mathrm{~min}$ at $4{ }^{\circ} \mathrm{C}$. The aqueous phase was then removed and combined with $500 \mu \mathrm{L}$ isopropanol, shaken vigorously, and rested at room temperature for $15 \mathrm{~min}$ to facilitate RNA precipitation. Samples were centrifuged at $12,000 \times \mathrm{g}$ for $15 \min$ at $4{ }^{\circ} \mathrm{C}$, and RNA pellets were washed with $1 \mathrm{~mL} \mathrm{70 \%} \mathrm{ethanol.} \mathrm{RNA} \mathrm{pellets} \mathrm{were} \mathrm{briefly} \mathrm{air} \mathrm{dried}$ and then suspended in $50 \mu \mathrm{L}$ RNase-free water. The quality of RNA was assessed by the appearance of two sharp bands representing $28 \mathrm{~S}$ and $18 \mathrm{~S}$ ribosomal RNA bands on a $1 \%$ agarose gel stained with SybrGreen dye (Appendix D). Purity was further assessed using a 260/280 nm ratio, and concentrations of RNA samples were determined and then standardized using a Take3 apparatus (BioTek, Winooski, VT).

\subsection{3 cDNA Synthesis}

Complementary DNA (cDNA) was synthesized from each RNA sample. In each reaction, $1 \mu \mathrm{g}$ of RNA was combined with $1 \mu \mathrm{L}$ of $200 \mathrm{ng} / \mu \mathrm{L}$ oligo-dT (5'TTTTTTтTтTтTтTTTTTTTTTV-3' where V=A, G, or C; Integrated DNA 
Technologies) in a total volume of $10 \mu \mathrm{L}$ autoclaved water. These samples were incubated at $65^{\circ} \mathrm{C}$ in an Eppendorf thermocycler for 5 minutes and then placed on ice to hybridize the oligo-dTs with the poly-A-tail of messenger RNA transcripts. Then, $4 \mu \mathrm{L}$ of $5 \mathrm{X}$ first strand buffer, $2 \mu \mathrm{L}$ of $0.1 \mathrm{M}$ dithiothreitol (DTT), $1 \mu \mathrm{L}$ of $10 \mathrm{mM}$ dNTPs, and 1 $\mu \mathrm{L}$ MMLV reverse transcriptase enzyme (Invitrogen) were added into each sample, and cDNA synthesis was induced by incubating samples at $42^{\circ} \mathrm{C}$ for 60 minutes in a thermocycler.

$\underline{\text { Primer design }}$

To design primers for relative quantification of specific genes in $X$. laevis cDNA samples, the sequences of genes corresponding to the $X$. laevis genome were obtained on the NCBI database (www.ncbi.nlm.nih.gov). Sequences of rat gene transcripts were obtained for $\operatorname{sirt} 1, \operatorname{sirt} 2, \operatorname{sirt} 3$, sirt4, sirt5, sirt6 sirt7, rpl.27, and gapdh. After accessing the mRNA sequence for the genes of interest, NCBI Primer-BLAST (http://www.ncbi.nlm.nih.gov/tools/primer-blast/) was used to produce potential primers which would not amplify with any other sequence present in the $X$. laevis transcriptome (Appendix C).

\subsubsection{RT-qPCR}

Gene targets were analyzed using a BioRad CFX96 Connect System (BioRad, Hercules, CA). Reagents were prepared for quantitative reverse transcription polymerase chain reaction (RT-qPCR) as previously described (Pellissier et al., 2006). Each reaction contained $2 \mu \mathrm{L}$ of $10 \mathrm{x}$ core buffer $(100 \mathrm{mM}$ Tris- $\mathrm{HCl}, \mathrm{pH} 8.5,500 \mathrm{mM} \mathrm{KCl}, 1.5 \%$ Triton $\mathrm{X}-100,20 \mathrm{mM} \mathrm{MgCl} 2,2 \mathrm{mM}$ dNTPs, and $100 \mathrm{nM}$ fluorescein), $6 \mu \mathrm{L}$ of $2 \mathrm{M}$ trehalose 
(BioShop, Canada), $0.5 \mu \mathrm{L}$ of $100 \%$ formamide (BioShop, Canada), $0.1 \mu \mathrm{L}$ of a 1:100 v:v SYBR green (Invitrogen) dilution in DMSO (BioShop, Canada), $1 \mu \mathrm{L}$ (1U) of Taq polymerase (BioShop, Canada), and primers (Integrated DNA Technologies; $250 \mathrm{nM}$ final concentration), and autoclaved water to a final volume of $20 \mu \mathrm{L}$. To perform RTqPCR, samples were incubated at $95^{\circ} \mathrm{C}$ for $3 \mathrm{~min}$, followed by 50 cycles of $95^{\circ} \mathrm{C}$ for 10 sec, $57^{\circ} \mathrm{C}$ for $20 \mathrm{sec}$, and $72^{\circ} \mathrm{C}$ for $20 \mathrm{sec}$. Serial dilutions of pooled cDNA samples were used to run standard curves of the primer sets in order to assess their specificity and efficiency (Appendix E). Every RT-qPCR reaction was analyzed with a melt curve analysis to ensure that the reaction was not amplifying nonspecific products.

\subsubsection{Protein extraction}

Protein extracts were prepared from 50-100 mg of frozen tissue by homogenization 1:3 w:v with a glass homogenizer in Lysis buffer (EMD Millipore, Cat. No. 43-010) combined with various phosphatase and protease inhibitors added immediately before use ( $1 \mathrm{mM} \mathrm{Na} \mathrm{VO}_{4}, 10 \mathrm{mM} \mathrm{NaF}, 10 \mathrm{mM} \beta$-glycerophosphate), and $10 \mu \mathrm{L} / \mathrm{mL}$ protease inhibitor cocktail (Cat. No. PIC001, BioShop). After homogenization, the samples were incubated on ice for $30 \mathrm{~min}$ with intermittent vortexing every $10 \mathrm{~min}$, and then centrifuged according to the manufacturer's guidelines at 10,000 rpm for $20 \mathrm{~min}$ at $4{ }^{\circ} \mathrm{C}$. The supernatant from each sample was collected, and protein concentration was determined using a Bradford protein assay (Bio-Rad, Cat. No. 500-0006). Protein concentrations were standardized for each sample to $10-20 \mu \mathrm{g} / \mu \mathrm{L}$ with lysis buffer and a subsequent Bradford assay was done to verify the concentrations. 


\subsubsection{Western immunoblotting}

Protein extracts prepared as above were combined with 2X SDS buffer (100 mM Tris-base, 4\% w:v SDS, $20 \%$ v:v glycerol, $0.2 \%$ w:v bromophenol blue, $10 \%$ v:v 2 mercaptoethanol, $\mathrm{pH}$ 6.8) in a 1:1 v:v ratio, boiled for $10 \mathrm{~min}$, and cooled on ice. Protein samples treated with SDS buffer were loaded onto 15\% SDS-polyacrylamide gels and run at $180 \mathrm{~V}$ for $60 \mathrm{~min}$ using a BioRad Mini-Protean 3 System. Gels were then transferred to $0.45 \mu \mathrm{m}$ PVDF membranes (EMD Millipore, Cat. No. IPVH00010) by electroblotting at a constant voltage of $30 \mathrm{~V}$ for $100 \mathrm{~min}$ in transfer buffer ( $25 \mathrm{mM}$ Tris-base, $\mathrm{pH} 8.5,192$ $\mathrm{mM}$ glycine, 10\% v:v methanol). Membranes were then washed in TBST (20 mM Trisbase, $\mathrm{pH}$ 7.6, $140 \mathrm{mM} \mathrm{NaCl}, 0.05 \% \mathrm{v}: \mathrm{v}$ Tween-20) for $3 \times 5 \mathrm{~min}$, before being blocked with $2.5 \% \mathrm{w}: \mathrm{v}$ powdered milk in TBST for $30 \mathrm{~min}$ at room temperature. Membranes were washed again for $3 \times 5 \mathrm{~min}$ in TBST, and probed with primary antibody diluted 1:1000 v:v TBST overnight at $4^{\circ} \mathrm{C}$. Primary antibodies targeting Human Ac-SOD (K68) (Abcam, Cat. No. ab137037) were used for this analysis. Membranes were washed again for $3 \times 5$ min in TBST, and probed with HRP-linked anti-rabbit IgG secondary antibody (Bioshop, Cat. No APA007P) diluted 1:8000 v:v in TBST for 30-60 min. Membranes were washed once more for $3 \times 5 \mathrm{~min}$ and visualized by enhanced chemiluminescence $\left(\mathrm{H}_{2} \mathrm{O}_{2}\right.$ and luminol) using a ChemiGenius Bio-Imaging System (Syngene, Frederick, MD). Following exposure, membranes were stained with Coomassie blue $(0.25 \% \mathrm{w}: \mathrm{v}$ Coomassie brilliant blue, $7.5 \% \mathrm{v}: \mathrm{v}$ acetic acid, $50 \%$ methanol) to visualize all protein bands. 


\subsubsection{Quantification of relative Sirt deacetylase activity}

Universal Sirt deacetylase activity was measured from cell lysates obtained from $X$. laevis tissues under control, medium dehydration, and high dehydration. All reagents for this assay were obtained from Epigentek (Cat. \#P-4036). Reactions were prepared by combining protein concentration-standardized cell lysates that were further diluted 1:10 in Sirt activity assay buffer with $1 \mu 1$ Sirt substrate (50X substrate), $1 \mu 1$ Sirt co-factor (50X NAD), and $1 \mu 1 \mathrm{HDAC}$ inhibitor (50 $\mu \mathrm{M}$ trichostatin A), to a total volume of $50 \mu \mathrm{l}$ in Sirt activity assay buffer. These reactions were placed in strip wells that were precoated by the manufacturer with a Sirt acetylated substrate, and incubated at $37^{\circ} \mathrm{C}$ for 1.5 $\mathrm{h}$ to promote Sirt activity. The dynamic range for each tissue type was determined using a pooled sample of control and dehydrated cell lysates. Following the incubation, reaction volumes were discarded and each well was washed three times with $150 \mu 1$ manufacturerprovided wash buffer. Using the manufacturer-provided capture antibody, a 1:1000 diluted capture antibody solution was prepared in wash buffer and was used to probe for deacetylated Sirt substrates bound to the reaction wells. In each well, $50 \mu 1$ of diluted capture antibody solution was added, before incubation at room temperature for $1 \mathrm{~h}$. The diluted capture antibody was then discarded and wells were washed again as previously. Using the manufacturer-provided detection antibody, a 1:2000 diluted capture antibody solution was prepared using wash buffer, $50 \mu 1$ of which was added to each well and then incubated for $30 \mathrm{~min}$ at room temperature. The capture antibody solutions were discarded and each well was then washed four times with wash buffer. For signal detection, $100 \mu \mathrm{l}$ of the manufacturer-provided Developer Solution was added to each well, and incubated $\sim 10-15$ min until the solutions became visibly blue. Reactions were then stopped with the 
addition of $100 \mu \mathrm{l}$ manufacturer-provided Stop Solution. Samples were measured at 450 $\mathrm{nm}$, with $655 \mathrm{~nm}$ as a reference wavelength. Reference-subtracted values were used for relative activity calculations since other variables such as protein amount and incubation time were kept constant in each comparison.

To further validate the assay, further wells were run where reactions with pooled samples were compared to those where pooled samples + a Sirt inhibitor (nicotinamide) were added. To prepare a sample containing a Sirt inhibitor, the manufacturer-provided nicotinamide was diluted 1:10 in Sirt activity assay buffer. Then, a reaction well was prepared as previously described using pooled cell lysates, except that $5 \mu$ of diluted Sirt inhibitor was also included in the $50 \mu 1$ reaction volume.

\section{$\underline{\text { 2.2.8 Quantification and statistics }}$}

Relative gene expression was determined with RT-qPCR in six tissues of $X$. laevis. The reference gene used was $r p l 27$, with the exception of heart, for which gapdh was used. All mRNA quantifications accounted for the primer efficiencies of every gene studied. Relative Sirt activity between control, medium, and high dehydration conditions was calculated by averaging $(450 \mathrm{~nm}-655 \mathrm{~nm})$ values for each experimental condition, and normalizing experimental values to the control, which was set to 1. Quantification of relative Sirt activity was performed with a protein amount that appears in the middle of the dynamic range so that the quantification was performed within the limit of detection, but also not at a point of saturation. Quantification of protein levels was performed with western immunoblotting, and subsequent analysis was done with a ChemiGenius BioImaging system (Syngene, Frederick, MD) and the accompanying GeneTools software. Band intensities from immunoblots were standardized against the total amount of protein represented by the intensity of Coomassie blue stained proteins on the membrane (Eaton 
et al., 2013; Welinder and Ekblad, 2011). Results from RT-qPCR, global Sirt deacetylase assays, and immunoblotting were expressed as mean \pm SEM. Statistically significant differences from the control were determined with a one-way ANOVA with Dunnett's post hoc test or Student's $t$-test, both of which were performed with RBioplot (Zhang and Storey, 2016). 


\subsection{Results}

\subsubsection{Dehydration-induced sirt mRNA gene expression}

Samples of six tissues (liver, skeletal muscle, brain, heart, lung, and kidney) from control and dehydrated $X$. laevis were assessed for differential sirtuin mRNA expression using RT-qPCR. In the liver, sirt1 increased with both medium and high levels of dehydration by $1.79 \pm 0.16$ and $2.22 \pm 0.26$ fold, respectively (Figure 2.2). Similarly, sirt4 transcripts also increased under both dehydration conditions by $2.39 \pm 0.31$ and $1.97 \pm 0.23$ fold, respectively. In contrast, sirt5 and sirt6 gene transcripts decreased in the high dehydration condition to $0.34 \pm 0.02$ and $0.65 \pm 0.01$ of control levels, respectively. Wholebody dehydration had no effect on the gene transcript levels of liver sirt2, sirt3, and sirt7.

Unlike the liver, skeletal muscle sirt1 mRNA levels were not affected by whole body dehydration and nor was sirt7 (Figure 2.3). However, muscle sirt2, sirt3, sirt4, sirt5, and sirt6 transcript levels were all responsive to dehydration. Medium and high dehydration states resulted in $2.41 \pm 0.43$ and $2.26 \pm 0.22$ fold increases in skeletal muscle sirt2 levels, respectively. High dehydration induced a $1.52 \pm 0.09$ fold increase in sirt3, and a decrease in sirt6 to $0.53 \pm 0.10$ of control levels. Interestingly, exposure to medium dehydration induced a decrease in muscle sirt4 and sirt5 to $0.69 \pm 0.04$ and $0.57 \pm 0.08$ respectively, an effect that was not observed in high dehydration.

In the brain, there was very little response by sirt mRNA expression to dehydration. High dehydration exposure resulted in a decrease in sirt 5 transcript levels to $0.57 \pm 0.08$ of control levels (Figure 2.4 ). However, transcript levels of all others did not change significantly with exposure to dehydration. 
Analysis of lung tissue from $X$. laevis under control and high dehydration conditions revealed that multiple sirt genes were upregulated during dehydration. Specifically, sirt1, sirt2, sirt6, and sirt7 increased by $1.84 \pm 0.22,3.79 \pm 0.77,1.76 \pm 0.26$, and $2.08 \pm 0.37$ fold with dehydration (Figure 2.6). However, dehydration reduced sirt5 transcript levels to $0.34 \pm 0.06$ of control levels

Transcript levels of all seven sirt genes were also assessed in the heart and kidney tissues of control and dehydrated $X$. laevis, but no significant changes in relative levels were observed (Figures 2.5 and 2.7).

\section{Global sirtuin deacetylase activity}

A global SIRT deacetylase activity assay was used to quantify the relative changes in total $\mathrm{NAD}^{+}$-dependent deacetylase activity of sirtuins present in tissue lysates of control and dehydrated $X$. laevis. Dehydration- increased global SIRT activity in liver, skeletal muscle, heart, and lung tissues of dehydrated X. laevis (Figure 2.8). Liver from medium and high dehydrated $X$. laevis exhibited $2.04 \pm 0.13$ and $1.55 \pm 0.07$ fold increases in global sirtuin activity, respectively, relative to control tissues. Lung also showed increased activities in both medium and high dehydration states, increasing $1.98 \pm 0.06$ and $2.33 \pm 0.25$ fold, respectively. Skeletal muscle demonstrated the highest increase in global sirtuin activity, which increased by $3.06 \pm 0.34$ fold with high dehydration, but activity between control and medium dehydration did not change. Heart also showed a modest, albeit significant, increase in global sirtuin deacetylase activity, rising by $1.26 \pm 0.07$ fold under high dehydration conditions. Global sirtuin activity did not significantly change with dehydration exposure in brain or kidney tissues. 


\subsubsection{Relative acetylation of SOD2 (K70) in dehydrating X. laevis}

The acetyl group on lysine 68 of mammalian superoxide dismutase 2 (the Mndependent mitochondrial form) is a known target for deacetylase activity by sirtuins (Chen et al., 2011; Qiu et al., 2010). A multi-species sequence alignment showed that the comparable residue in the $X$. laevis SOD2 sequence was K70 (Figure 2.9). Immunoblotting with a primary antibody targeting human Ac-SOD2 (K68) was used to characterize the acetylation status of this conserved amino acid residue in six tissues of dehydrated X. laevis (Figure 2.10). In heart, SOD2 acetylation decreased to $0.39 \pm 0.08$ of control values under high dehydration, although levels did not significantly change in medium dehydration (Figure 2.11). In kidney, relative Ac-SOD2 content decreased under both medium and high dehydration states to $0.66 \pm 0.07$ and $0.46 \pm 0.10$ of control values, respectively (Figure 2.10). However, the greatest decrease in Ac-SOD2 levels occurred in lung, where exposure to medium and high dehydration resulted in decreases to $0.32 \pm 0.04$ and $0.22 \pm 0.03$ of control levels, respectively. 


\subsection{Discussion}

The present chapter characterized a family of sirtuins, $\mathrm{NAD}^{+}$-dependent protein deacetylases, in six tissues of $X$. laevis under control, medium dehydration, or high dehydration states. As the expression of sirt genes is known to occur in response to a number of different cellular processes, each of the 7 sirt genes were evaluated individually using RT-qPCR. Relative global sirtuin deacetylase activities were also measured in tissues from X. laevis exposed to different levels of whole-body dehydration. Together, these results suggested an overall increase in sirtuin signaling at the transcriptional and deacetylase activity levels in dehydrating $X$. laevis. Furthermore, assessment of Ac-SOD2 (K70), a known target for deacetylase activity was assessed suggested that regulation by post-translational acetylation during whole body dehydration may play a role in the activation of antioxidant signaling in X. laevis.

\subsubsection{Regulation of sirt transcript levels in response to dehydration}

Increases in sirtl transcript levels were observed in liver and lung tissues with exposure to whole-body dehydration (Figures 2.2 and 2.6). In rodent models of oxidative stress, SIRT1 has been shown to be a crucial activator of the mitochondrial antioxidant system (Figure 2.12), as evidenced by increases in antioxidant enzymes like SOD2 (Wang et al., 2015). The present results are consistent with previous work that showed a FoxO1 transcription factor-mediated increase in SOD2 mRNA and protein levels in the livers of dehydrating X. laevis (Malik and Storey, 2011). Indeed, other groups have demonstrated that both SIRT1 and FoxO transcription factors are crucial for cell survival under oxidative stress conditions. Using a cell line model, one group demonstrated that exposure to resveratrol (a SIRT1 activator) resulted in SOD2 expression, reduced reactive 
oxygen species, and an increased anti-apoptotic response (Hori et al., 2013). Furthermore, it has been shown that reversible protein acetylation plays an equally important role to that off reversible protein phosphorylation with respect to the control of FoxO transcriptional activity. In one study, it was shown that acetylation of FoxO1 proteins played an inhibitory role that attenuated DNA binding, and also facilitated inhibitory phosphorylation of protein residues (Matsuzaki et al., 2005). However, activation of sir2 (the yeast homolog of the sirt1 gene) promoted FoxO1-mediated transcription through its deacetylase activity (Daitoku et al., 2004). Together, the results and the literature demonstrate a role for post-translational acetylation control by sirt1 in whole-body dehydration of $X$. laevis, which contributes to the increased expression of antioxidants in the liver, and potentially the lungs.

The results also showed an increase in sirt 2 and $\operatorname{sirt} 3$ mRNA expression in skeletal muscle, with sirt2 also increased in lung tissue of dehydrated $X$. laevis (Figures 2.3 and 2.6). Sirt2 and sirt3 are also known to play roles in facilitating antioxidant responses. In rodent striatum tissue and fibroblast cell cultures, sirt2 knockout resulted in a significantly increased GSSG/GSH ratio (Liu et al., 2017). Reduced glutathione (GSH) is an important free radical scavenger that becomes increasingly abundant in its oxidized (GSSG) form in environments of lower antioxidant capacity or high oxidative stress (Zitka et al., 2012). An increase in sirt2 transcription may signify an activation of antioxidant responses through glutathione signaling in the skeletal muscle and lung tissues of $X$. laevis. In the skeletal muscle, sirt3 rose during dehydration (Figure 2.3). Not only is sirt3 responsible for regulating antioxidant responses, but it also deacetylates many other mitochondrial proteins, since it is the most abundant sirtuin in the 
mitochondrion (Pantazi et al., 2013). With its deacetylase activity, SIRT3 enhances the activity of SOD2 (Chen et al., 2011; Qiu et al., 2010), and regulators of the glutathione system (Pantazi et al., 2013; Someya et al., 2010). In one example, SIRT3 deacetylated the regulating FoxO3 transcription factor, resulting in its localization to the nucleus where it increased antioxidant capacity by promoting the transcription of SOD2 and catalase (Jacobs et al., 2008; Sundaresan et al., 2009). Models of sirt3 knockouts also exhibit hyperacetylation of mitochondrial proteins, which is not surprising given that it is the primary mitochondrial sirtuin (Pantazi et al., 2013). In addition to antioxidant enzymes, SIRT3 has also been shown to deacetylate mitochondrial HSPs, namely HSP60 (Jing et al., 2011). Although the mechanism of acetylation of HSPs warrants further study, these findings suggest that HSPs may have a role in the response to whole body dehydration.

Dehydration also induced an upregulation in sirt4 in X. laevis liver tissues (Figure 2.2). Unlike the other sirtuins discussed thus far, SIRT4 is not known for regulating antioxidant responses. In a study that knocked down sirt4 in primary mouse hepatocytes in vitro, SIRT4 suppression resulted in an increased mRNA expression of mitochondrial and fatty acid metabolism enzymes such as medium-chain acyl-CoA ( $m c a d)$, carnitine palmitoyl transferase 1a (cpt1a), PPARgamma coactivator 1 alpha $(p g c-1 \alpha)$ and peroxisome proliferator-activated receptor alpha (ppara) (Nasrin et al., 2010). This was repeated in an in vivo model and results for knocking down SIRT4 were congruent in that transcript levels for enzymes involved in fatty acid oxidation were increased (Nasrin et al., 2010). In both in vitro and in vivo models, suppression of sirt4 resulted in increased fatty acid oxidation (Nasrin et al., 2010). From these previous studies, an increase in sirt4 
signaling in the liver of dehydrated $X$. laevis may suggest that fatty acid oxidation is suppressed in favour of other energy producing pathways, such as glycolysis (Figure 2.13). Due to the impairment of oxygen transport caused by whole-body dehydration in X. laevis, it is possible that the use of fatty acid substrates becomes less favoured. Although these results warrant further investigation into the state of fatty acid oxidation pathways in dehydrating $X$. laevis, the data suggest that sirtuins regulate multiple facets of metabolism during whole-body dehydration.

Another sirtuin with metabolic functions is SIRT5, which plays a significant role in nitrogen metabolism (Figure 2.13). One important role of SIRT5 is the deacetylation and activation of carbamoyl phosphate synthetase 1 (CPS1), which catalyzes the first step of the urea cycle (Nakagawa et al., 2009). An upregulation in liver of sirt5 transcript levels during dehydration could be expected, given that naturally aestivating X. laevis, collected from the mud in dried-up pools, exhibited 15-20 fold increases in tissue urea concentrations, and a 6-fold increase in CPS1 activity (Balinsky et al., 1967). Although the animal treatment conditions of the present study build on previous work with dehydrated X. laevis in standard laboratory conditions that do not involve naturally aestivation in nature, animals in both cases are known to accumulate significant amounts of tissue and plasma urea (Balinsky et al., 1961; Malik and Storey, 2009). Due to the known role of SIRT5 in controlling the urea cycle, it would be expected that sirt5 transcription would be activated in liver of dehydrating X. laevis. Unexpectedly, however, sirt 5 transcript levels in liver decreased strongly in dehydrated X. laevis (Figure 2.2). However, it is important to note that global SIRT deacetylase activity in the liver significantly increased during dehydration (Figure 2.8). Although the results suggest that 
liver sirt5 is not a gene that is actively transcribed during dehydration, an increase in SIRT5 deacetylase activity cannot be ruled out at this point.

Transcript levels of sirt6 increased in the lungs during dehydration (Figure 2.6). This is yet another sirtuin that plays a role in the activation of cytoprotective pathways (Figure 2.12). Unlike the other sirtuins previously discussed, SIRT6 contributes to cell survival by localizing to the promoter regions of the NRF2 transcription factor, where it directly recruits RNA polymerase II and facilitates transcription by deacetylating Histone 3 at K56 (Pan et al., 2016). Furthermore, this interaction results in the expression of heme oxygenase (HO-1), a downstream antioxidant gene of NRF2 (Pan et al., 2016). Reduced SIRT6 has also been shown to aggravate oxidative stress in rodent models of ischemia/reperfusion. This rodent model of injury is relevant to whole-body dehydration because an increase in blood viscosity and increased hematocrit can lead to an impedance in circulation. Decreasing SIRT6 in in vivo liver models of ischemia/reperfusion resulted in reduced relative SOD activity, increased necrotic tissue, decreased anti-apoptotic Bcl-2 protein, increased pro-apoptotic Bax protein, and increased cleaved (activated) apoptosispromoting caspase-3 protein (Zhang et al., 2018). Another study using an in vivo ischemia/reperfusion model of the heart also demonstrated that SIRT6 provided a cardioprotective effect through the expression of antioxidants SOD2 and catalase via FoxO3-activated gene transcription (Wang et al., 2016), similar to the action of SIRT3 discussed earlier.

Lastly, sirt7 transcription increased in response to dehydration in X. laevis lung (Figure 2.6). A study on pulmonary fibrosis revealed that this condition caused a decrease in all Sirt mRNA levels, with corresponding changes in protein levels (Wyman et al., 
2017). However, the largest decrease associated with pulmonary fibrosis was for SIRT7. Further experiments showed that inhibition of SIRT7 in lung fibroblasts resulted in increased collagen and $\alpha$-smooth muscle actin ( $\alpha$-SMA), and reciprocally, overexpression of SIRT7 resulted in lower collagen and $\alpha$-SMA mRNA levels, in addition to reduced Smad3 mRNA and protein (Figure 2.14) (Wyman et al., 2017). Through bouts of dehydration and rehydration, the lungs of $X$. laevis can be considered to undergo significant stress since these are responsible for a higher proportion of gas exchange as compared to the skin. An increase in sirt7 expression in the lungs of X. laevis may highlight a cytoprotective role played by sirtuin deacetylases that reduces scarring of the lungs caused by periods of dehydration and rehydration.

\subsubsection{Correlations with global SIRT deacetylase activity}

Measurements of relative SIRT activity in X. laevis tissues under control and dehydrated conditions demonstrated an overall increase in $\mathrm{NAD}^{+}$-dependent deacetylase activity, specifically in the liver, skeletal muscle, heart, and lung tissues (Figure 2.9). These results consistently correlated with the increased transcript levels of sirt genes found during dehydration and with known actions of various SIRT isozymes in activating antioxidant and/or cytoprotective responses, or suppressing fatty acid oxidation signaling (Table 2.1). However, the results from the deacetylase activity assays reflect very little on the activity of SIRT4, since this protein is known to perform ADP-ribosyltransferase activity, and its deacetylase activity is negligible. Furthermore, despite the general increase in liver SIRT activity which is consistent with increased urea production through the deacetylation and activation of CPS1, the observed decreased sirt 5 mRNA data was not expected to occur (Figure 2.2) (Nakagawa et al., 2009). This suggests that the cellular 
pathways that regulate metabolic processes likely involve other levels of regulation, potentially by posttranscriptional regulation by non-coding RNAs. The present analyses highlight the particular sirt genes that showed increased expression due to whole-body dehydration, which may signify the importance of these selected sirtuins in dealing with dehydration since energy expenditure would need to be committed to facilitate their transcription (and presumably also their translation). However, the activity data cannot provide a direct measure of increases or decreases in relative deacetylase activity of individual sirtuin proteins. Thus, despite reduced sirt 5 mRNA levels observed in liver, for example, the possibility of increased liver SIRT5 deacetylase activity cannot be ruled out. This principle applies for all tissues; for example, in heart, global sirtuin activity increased during dehydration, but no change in transcript levels of any of the sirtuin genes were observed (Figures 2.5 and 2.9). However, together with the dehydrationresponsive increases in sirt mRNA expression, results from the global Sirt activity assay suggest that post-translational acetylation plays a role in the molecular response to wholebody dehydration in $X$. laevis.

\subsubsection{Regulation of the SIRT substrate Ac-SOD (K70)}

In order to further explore the potential roles of sirtuin deacetylase activity in dehydrating $X$. laevis, a SIRT deacetylase substrate, acetylated SOD2 protein, was investigated via immunoblotting in 6 tissues under control, medium dehydration, and high dehydration conditions. In mammals, it is known that the acetylation of SOD2 on the K68 residue has an inhibitory effect, which can be relieved by SIRT3 deacetylation, thereby leading to increased SOD2 activity (Chen et al., 2011; Qiu et al., 2010). In $X$. laevis, the acetylated K68 residue is conserved, but exists as K70 (Figure 2.9). In 
response to dehydration, levels of K70 acetylation in $X$. laevis were suppressed in heart and kidney, but most strongly in the lungs (Figures 2.10 and 2.11). Interestingly, sirt3 transcript levels did not increase in these tissues during dehydration (Figures 2.5, 2.6 and 2.7). Furthermore, a decrease in Ac-SOD (K70) was not observed in skeletal muscle (Figures 2.10 and 2.11), a tissue that showed elevated sirt3 transcript levels (Figure 2.3) as well as a global increase in SIRT deacetylase activity (Figure 2.8). However, significant reductions in SOD2 acetylation in the heart and lung (Figures 2.10 and 2.11) both correlated with increased in global SIRT activity in these tissues (Figure 2.9). According to current literature, the observed decrease in acetylated SOD2 in kidney of $X$. laevis is likely due to SIRT3 deacetylase activity (Chen et al., 2011; Qiu et al., 2010). If this is the case, then a potential limitation must be noted in that the results from the global SIRT deacetylase assay, which did not identify any relative change in overall SIRT deacetylase activity in kidney, may fail to detect significant changes in individual SIRT deacetylases that may be occurring. Overall, the results indicate that $X$. laevis dehydration involves reversible protein acetylation as a regulatory mechanism to control crucial processes such as antioxidant responses.

\subsection{Conclusions}

This chapter showed that differential expression of sirt mRNA transcripts occurred in response to whole body dehydration in $X$. laevis in a tissue-specific manner. Furthermore, increased global activity of SIRT deacetylases in four tissues also supported the idea that enhanced sirtuin gene expression was leading to increased SIRT protein and SIRT activity in response to dehydration. By identifying the specific sirtuins that underwent dehydration-induced transcription, along with the global sirtuin deacetylase 
activity of each tissue, insights into the potential roles of SIRTs during dehydration could be made. The transcript levels of sirt 1, 2, 3 and 6, were all elevated in response to dehydration. These four sirtuins are known to activate antioxidant defense signaling through various pathways (Figure 2.12). Furthermore, they were upregulated in the liver, skeletal muscle, and lung tissues, which also all demonstrated increased global SIRT deacetylase activity with dehydration exposure (Figure 2.8). Transcript levels of sirt4 also increased in the liver, and although SIRT4 is known to exhibit negligible deacetylase activity, it was previously demonstrated to suppress fatty acid oxidation and the expression of genes required for mitochondrial activity (Figure 2.13), both of which could be important under dehydrating conditions. Although sirt5 transcript levels were not increased in response to dehydration, it cannot be ruled out that its deacetylase activity was not included among the increase in global SIRT activity in the liver (Figure 2.8). In the liver, SIRT5 activity is responsible for deacetylating and activating CPS1, the first step into the urea cycle (Figure 2.13). Since CPS1 and urea levels have been shown previously to increase significantly in wild aestivating and lab dehyhdrated $X$. laevis, liver cellular pathways that regulate the urea cycle warrant further study. Increased in sirt7 transcript levels correlated with increased Sirt deacetylase activity in lung, which is opposite to what is observed in pulmonary fibroblasts that are susceptible to fibrosis. This result suggests a sirt7-dependent mechanism of pulmonary cytoprotection that may assist X. laevis through bouts of dehydration (Figures 2.14). Lastly, the relative abundance of Ac-SOD2 (K70), a known substrate of Sirt3 deacetylase action, was found to decrease in the heart, lung, and kidney tissues under dehydration (Figure 2.11). These reductions in Ac-SOD2 abundance correlated with increased global Sirt deacetylase activity in these 
tissues, and underscore a deacetylation-dependent mechanism for regulating an antioxidant response during $X$. laevis dehydration. Overall, the results from this chapter demonstrate that sirtuins are actively transcribed during $X$. laevis dehydration in a tissuespecific manner leading to the upregulation of Sirt NAD ${ }^{+}$-dependent activity that, in turn, can have actions that may activate antioxidant and cytoprotective responses, as well as facilitate remodelling of fatty acid and urea metabolism. 


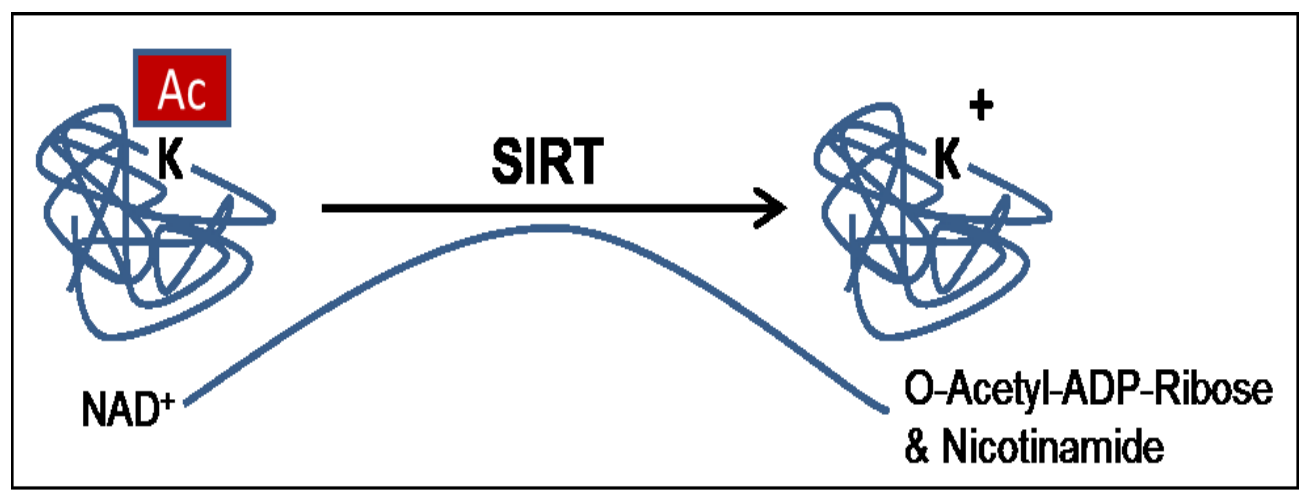

Figure 2.1. Sirtuin deacetylase activity involves the hydrolysis of $\mathrm{NAD}^{+}$into O-AcetylADP-Ribose and nicotinamide in order to deacetylase a lysine residue. 


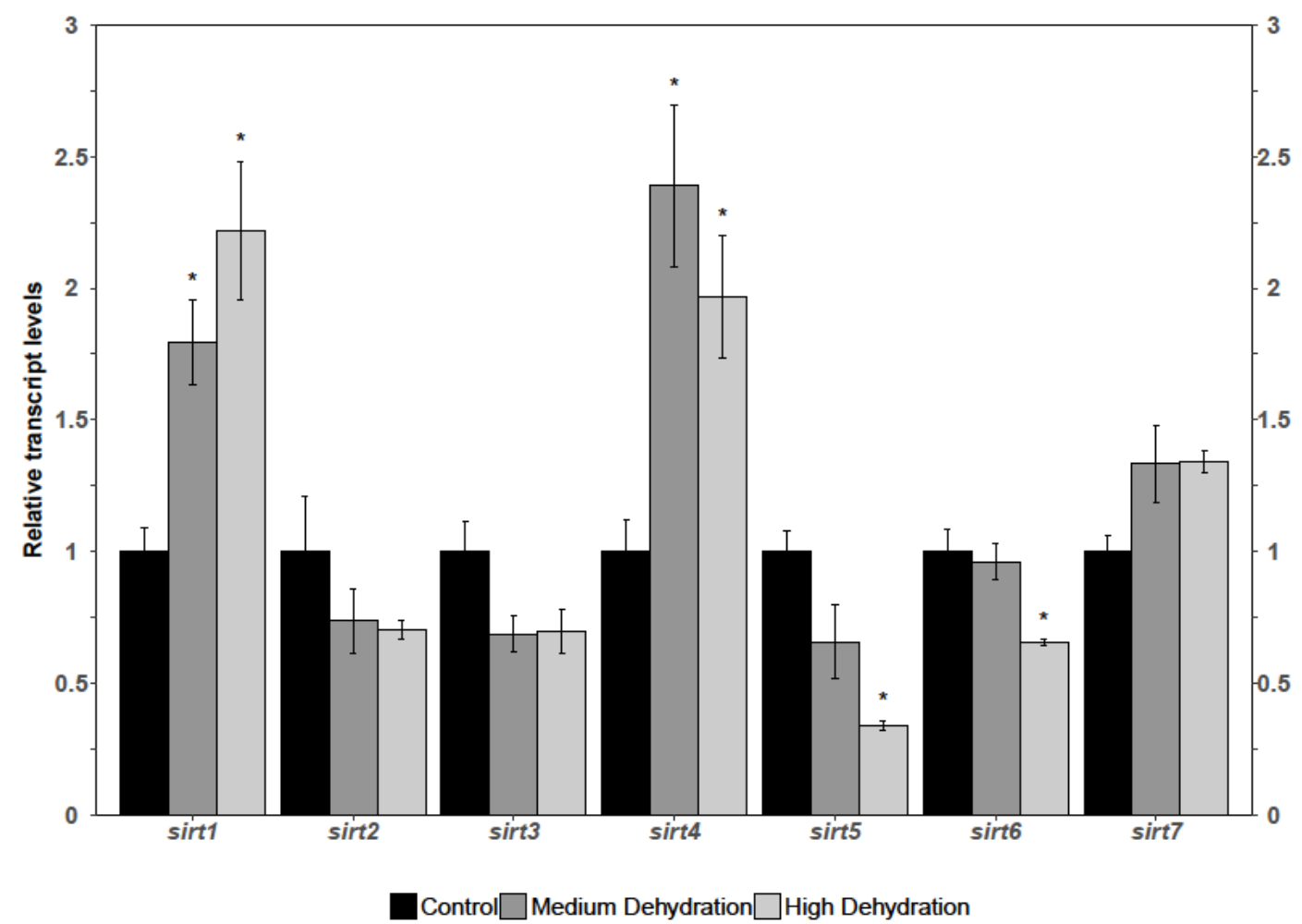

Figure 2.2. Relative mRNA expression of 7 sirt genes was assessed in liver using RTqPCR to investigate the effects of medium and high levels of whole-body dehydration of $X$. laevis. Gene expression data were standardized against $r p l 27$ as a reference gene. Data are means $\pm \mathrm{SEM}, n=4$ independent biological replicates. Statistically significant differences compared to the control condition are denoted with an asterisk $(*)$ and were determined with a one-way ANOVA and post hoc Dunnett's test $(p<0.05)$. 


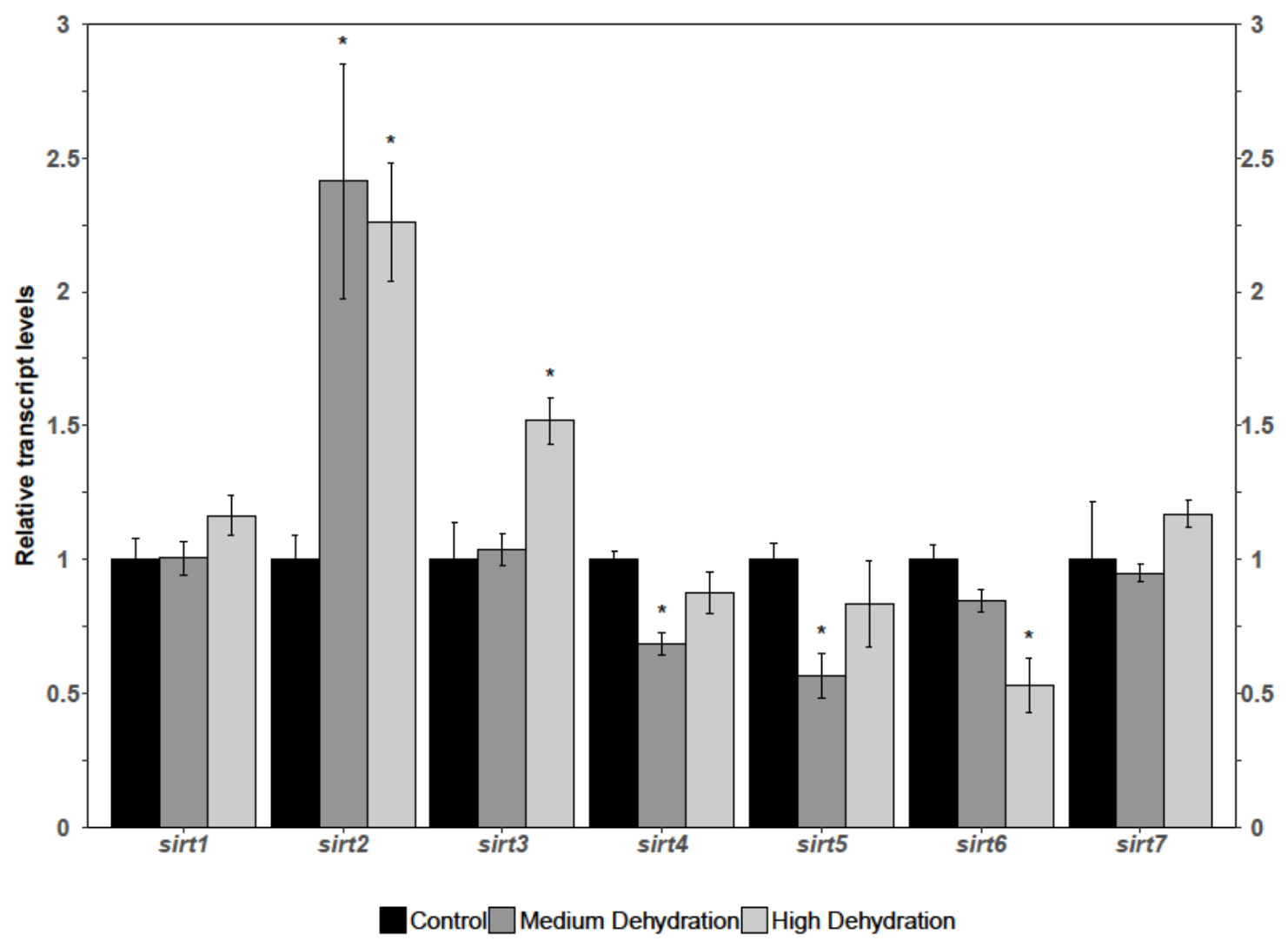

Figure 2.3. Relative mRNA expression of 7 sirt genes was assessed in $X$. laevis skeletal muscle using RT-qPCR to investigate the effects of medium and high levels of wholebody dehydration. Gene expression data were standardized against $r p l 27$ as a reference gene. Other information as in Figure 2.2. 


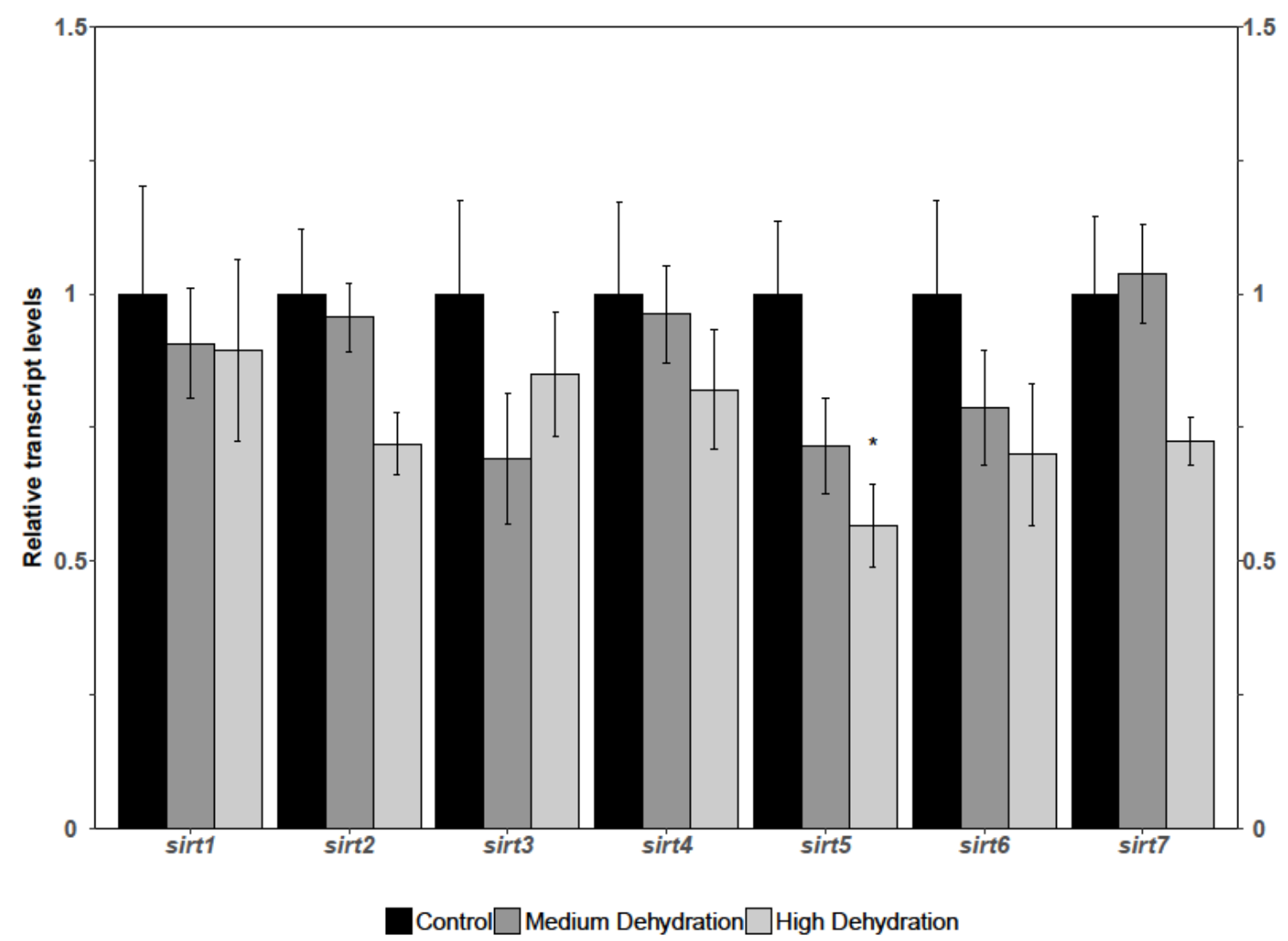

Figure 2.4. Relative mRNA expression of 7 sirt genes was assessed in X. laevis brain using RT-qPCR to investigate the effects of medium and high levels of whole-body dehydration. Gene expression data were standardized against $r p l 27$ as a reference gene. Other information as in Figure 2.2. 


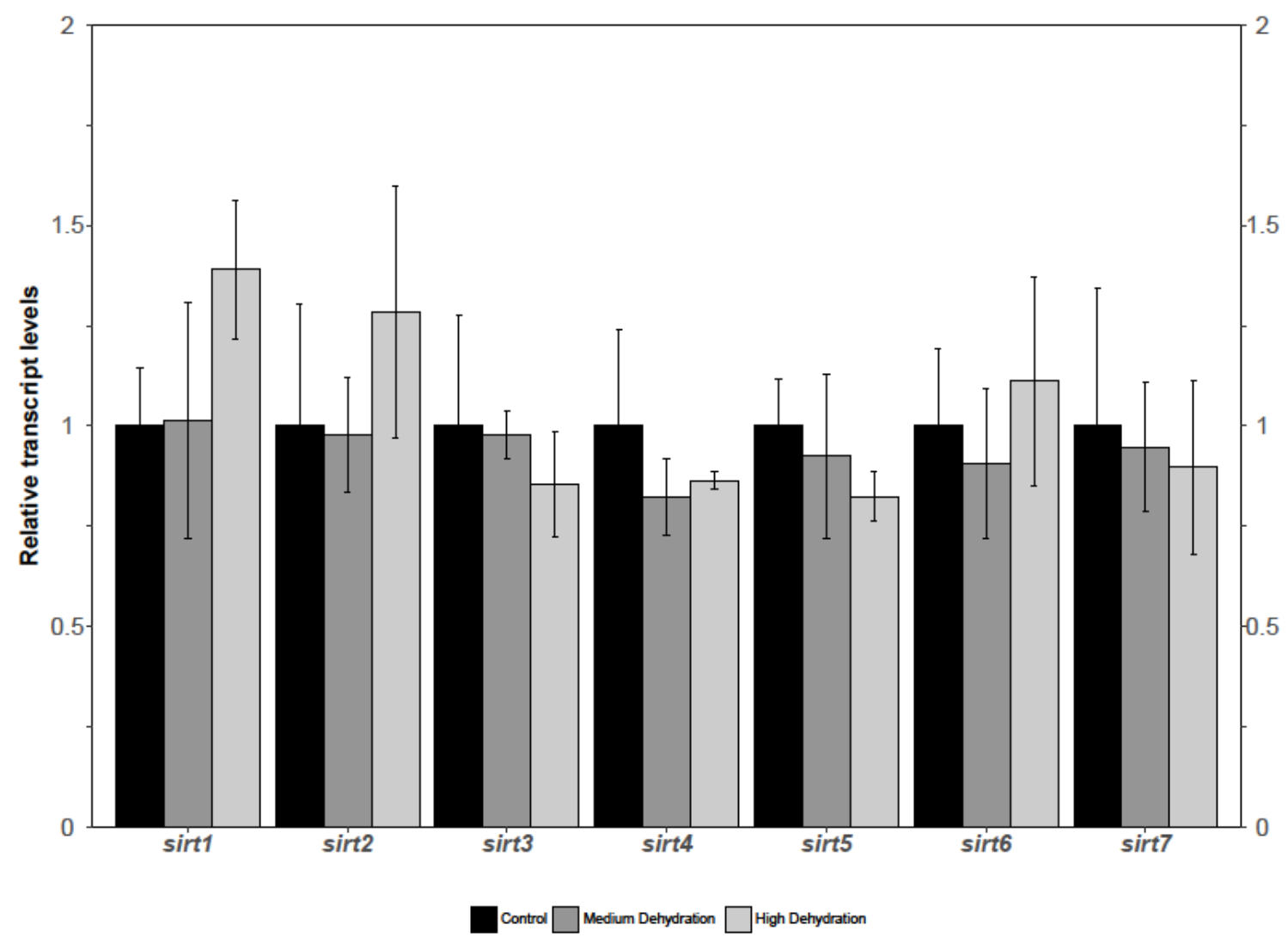

Figure 2.5. Relative mRNA expression of 7 sirt genes was assessed in $X$. laevis heart using RT-qPCR to investigate the effects of medium and high levels of whole-body dehydration. Gene expression data were standardized against gapdh as a reference gene. Other information as in Figure 2.2. 


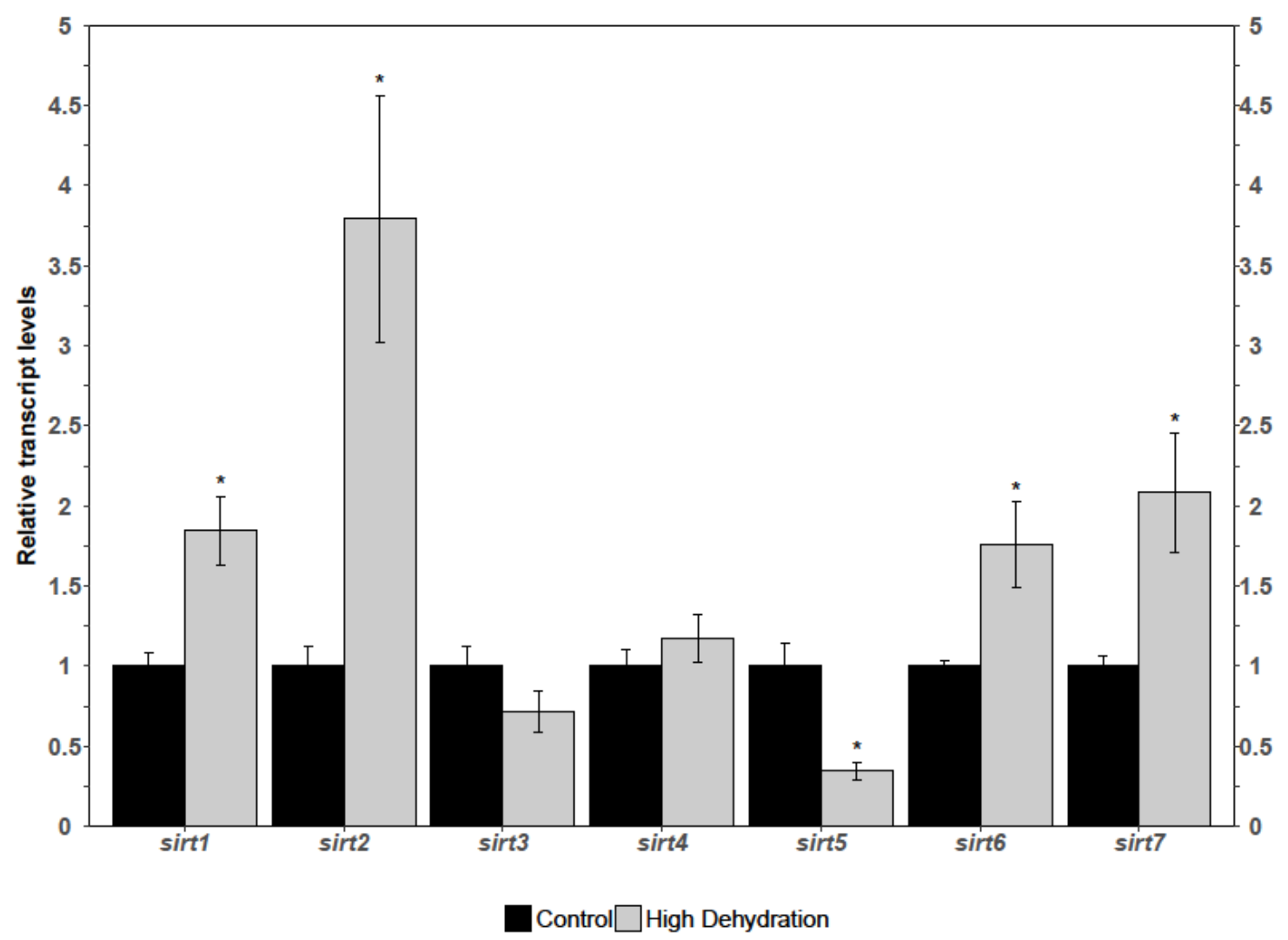

Figure 2.6. Relative mRNA expression of 7 sirt genes was assessed in lungs using RTqPCR to investigate the effects of whole-body dehydration of $X$. laevis at high dehydration levels. Gene expression data quantified from lung tissues were standardized against $r p l 27$ as a reference gene. Other information as in Figure 2.2, except that $\boldsymbol{n}=\mathbf{6}$ independent biological replicates. 


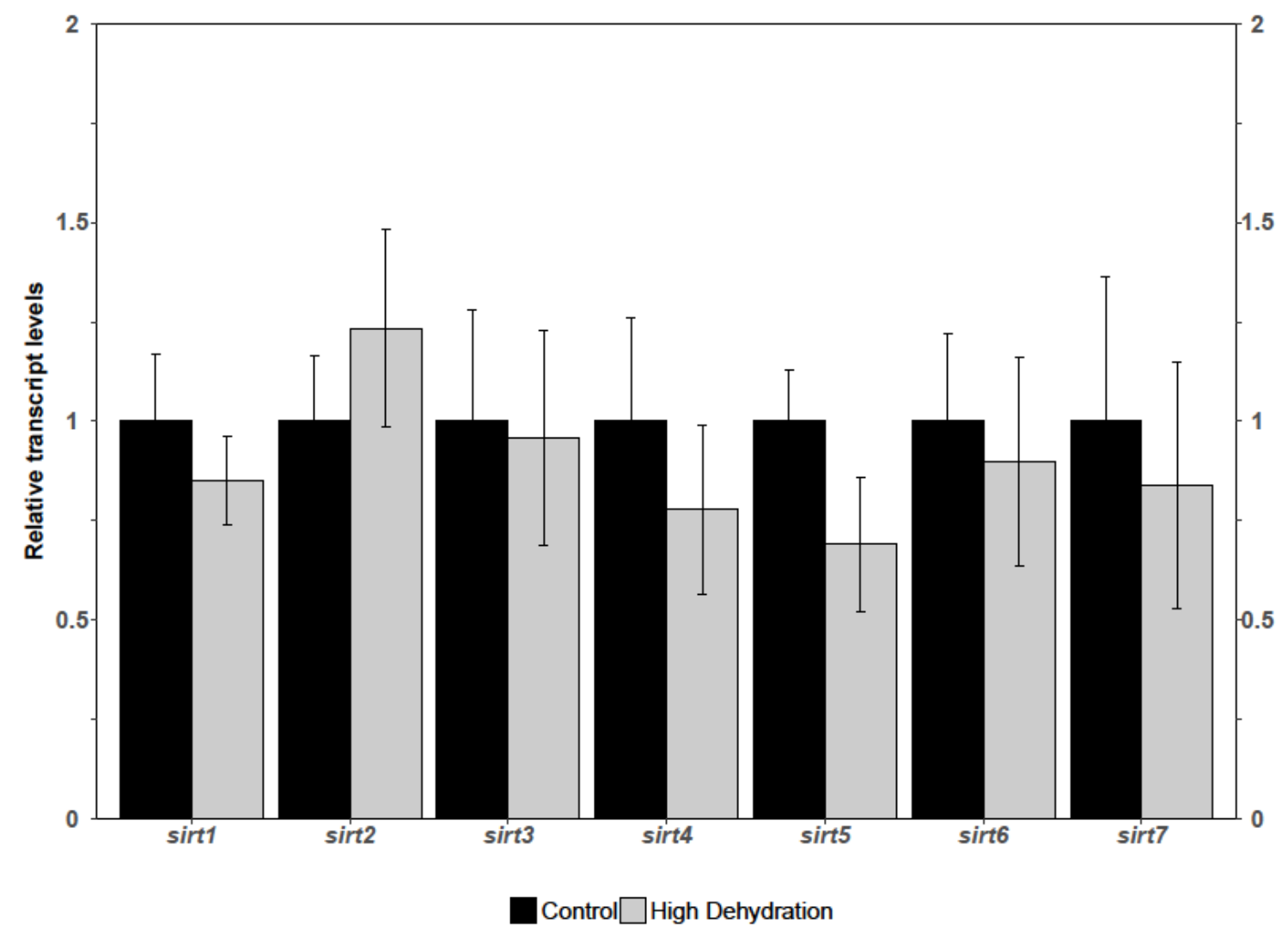

Figure 2.7. Relative mRNA expression of 7 sirt genes was assessed in kidneys using RTqPCR to investigate the effects of whole-body dehydration of $X$. laevis at high dehydration levels. Gene expression data quantified from kidneys tissues were standardized against $r p l 27$ as a reference gene. Other information as in Figure 2.2, except that $\boldsymbol{n}=\mathbf{8}$ independent biological replicates. 


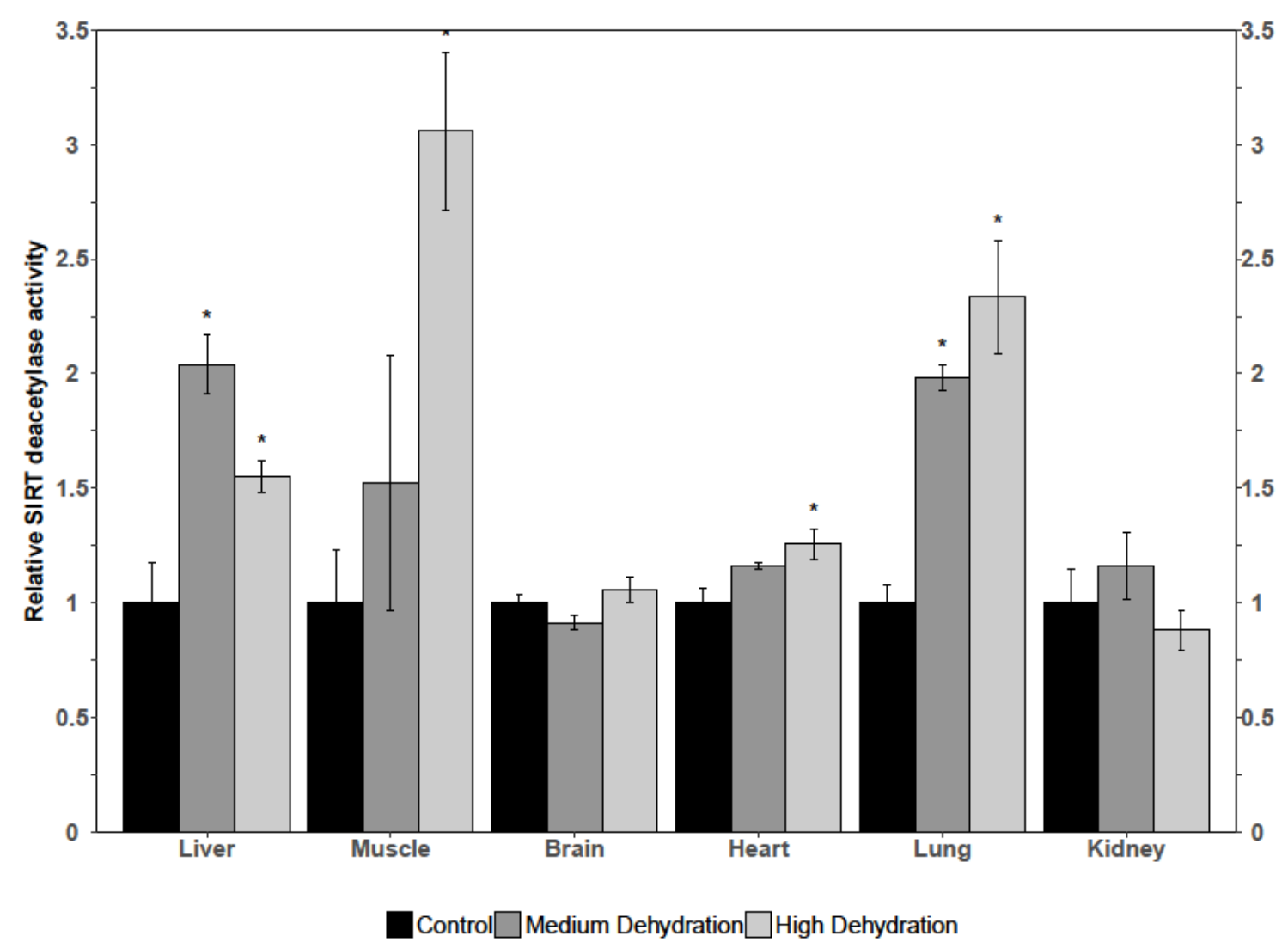

Figure 2.8. A global Sirt activity assay was used to determine the relative SIRT deacetylase activity in X. laevis tissues exposed to dehydration conditions. Enzyme activities from cell lysates are expressed as relative activity (arbitrary units) for normalized protein amounts. Histograms show means \pm SEM from $n=4$ independent biological replicates. Statistically significant differences between dehydrated conditions compared to the controls are denoted with an asterisk $(*)$ and were determined with a one-way ANOVA and post hoc Dunnett's test $(p<0.05)$. 


\section{H.sapiens \\ X.laevis \\ M.musculus \\ R. norvegicus}

\section{VNNLNVTEERYQEALAKG VNNLNITEEKYAEALAKG VNNLNATEEKYHEALAKG VNNLNVTEEKYHEALAKG \\ $* * * * * \quad * * * * * * * * * * *$}

Figure 2.9. The acetylated lysine 68 residue on SOD2 of humans, identified by a red box, is conserved in other mammals and in X. laevis. The SOD2 K68 residue occurs in all three mammalian sequences, but is K70 in $X$. laevis. Primary structures of SOD2 for all animals were obtained from NCBI Protein, and were aligned with EMBL-EBI Clustal Omega. Only a segment of the full primary sequence is shown. 


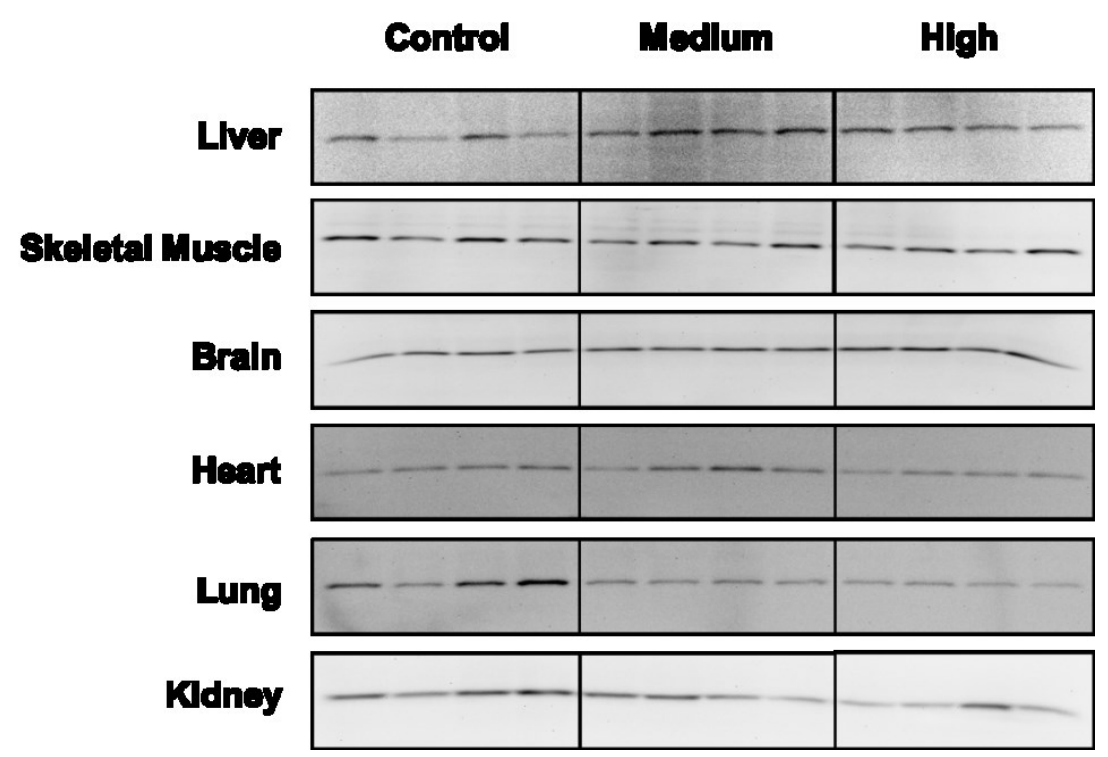

Figure 2.10. Immunoblots depicting relative changes in the amount of acetylated SOD2 (K70) in X. laevis tissues. Dehydration led to reduced amounts of acetylated SOD2 protein in heart, lung, and kidney tissues. 


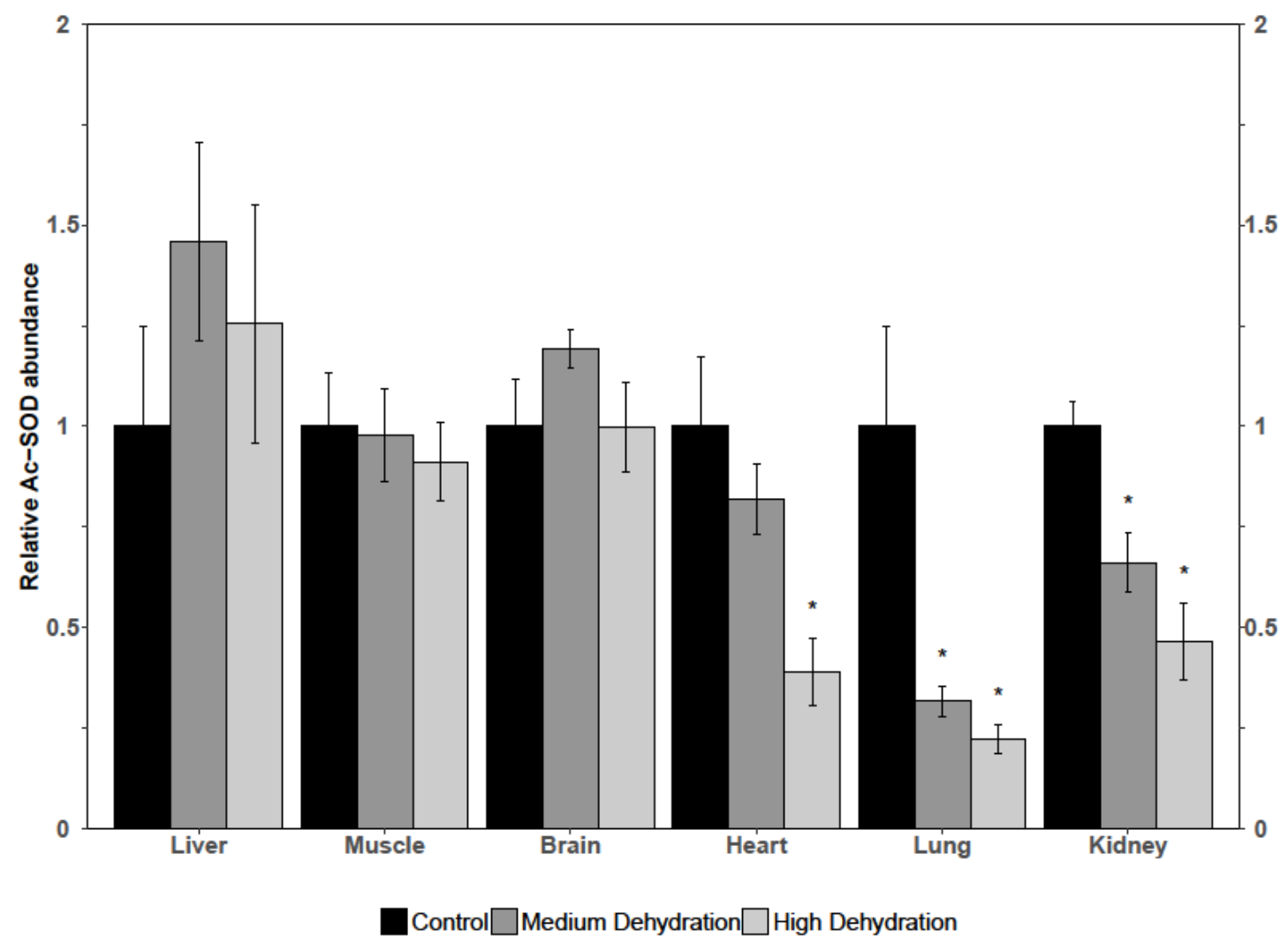

Figure 2.11. Relative abundance of $X$. laevis acetylated SOD2 (K70) protein in six tissues under control, medium, and high dehydration states. Histograms show means \pm SEM from $n=4$ independent biological replicates. Statistically significant differences between dehydrated conditions compared to the controls are denoted with an asterisk $\left(^{*}\right)$ and were determined with a one-way ANOVA and post hoc Dunnett's test $(p<0.05)$. 


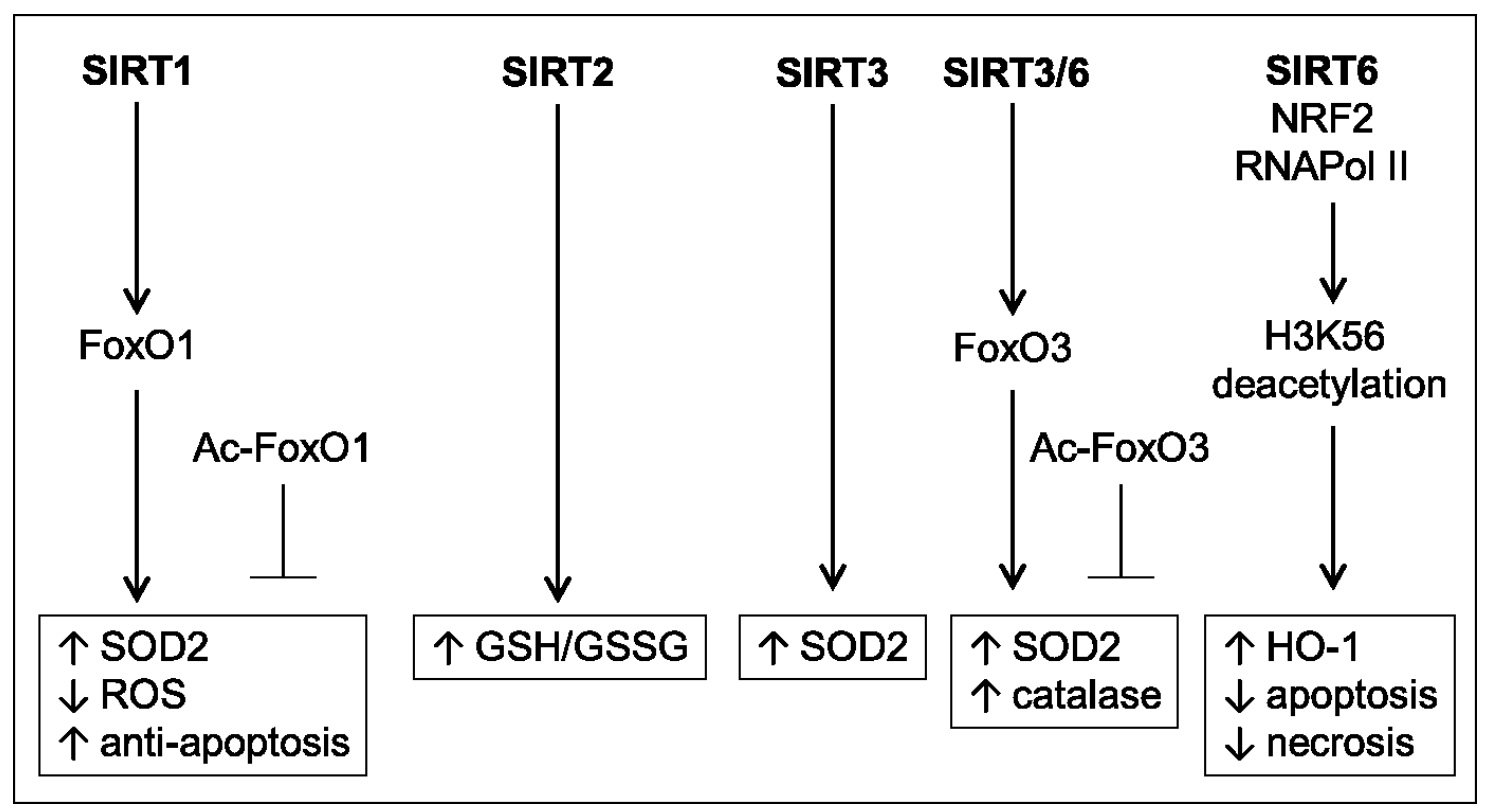

Figure 2.12. Roles of various Sirts in the regulation of antioxidant response signaling. 


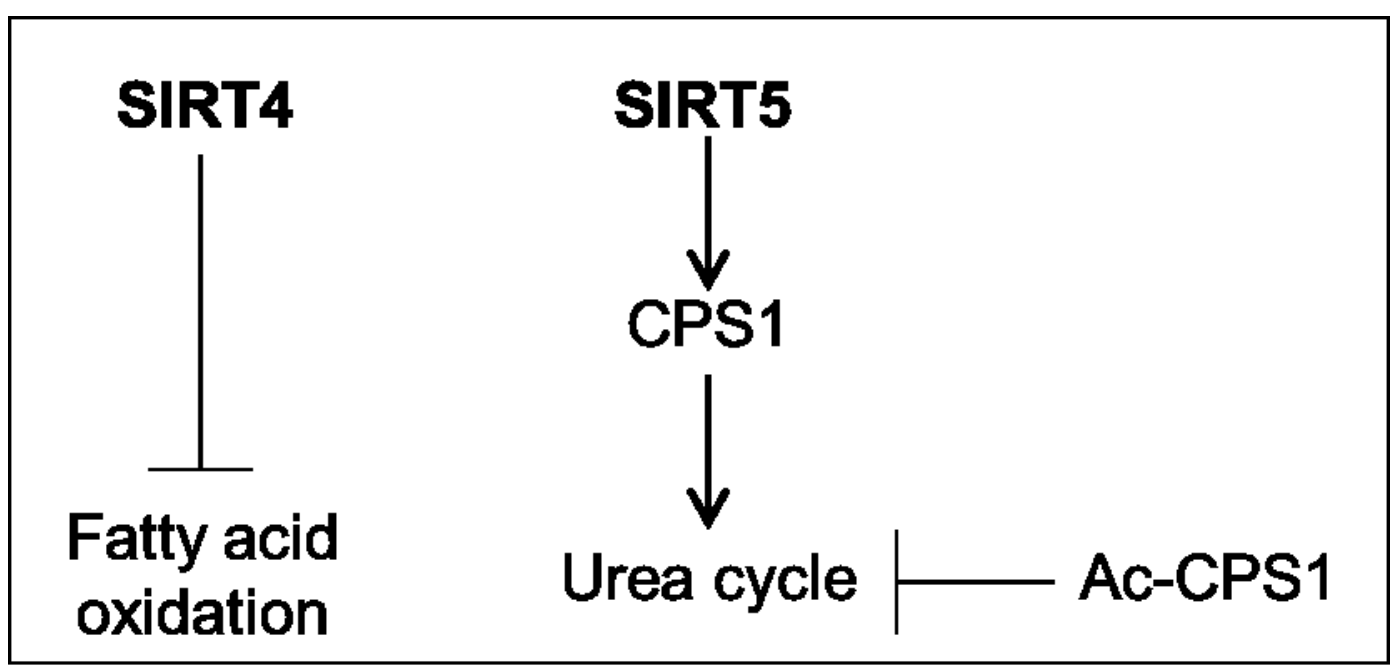

Figure 2.13. The SIRT4 and SIRT5 proteins have metabolic roles in the cell. SIRT4 is known to repress fatty acid oxidation, and SIRT5 activates the urea cycle by deacetylating and activating CPS1. 


\section{Pulmonary fibrosis}

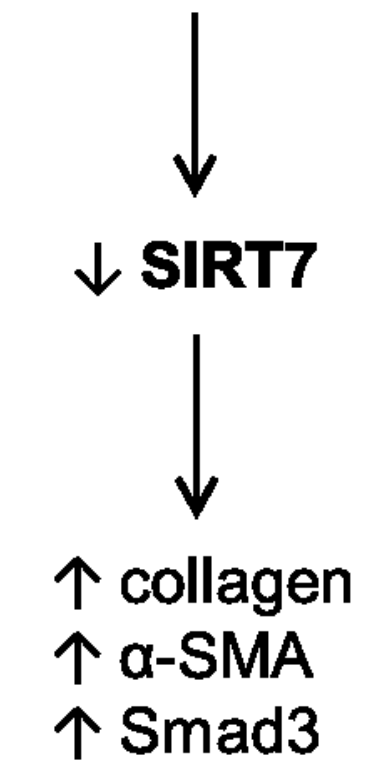

Figure 2.14. In pulmonary fibrosis, a suppression of all 7 SIRT proteins is observed, but particularly SIRT7. Suppression of SIRT7 during pulmonary fibrosis is known to facilitate an upregulation of collagen, $\alpha$-SMA, and Smad3. Oppositely, overexpression of SIRT7 in fibroblasts results in decreased collagen, $\alpha$-SMA, and Smad3 expression, which are genes that are characteristic of pulmonary fibrosis. 
Table 2.1 A summary of the characterization of Sirts and their potential roles in response to whole-body dehydration in $X$. laevis

\begin{tabular}{|c|c|c|}
\hline Sirtuin & $\begin{array}{l}\text { Increase in } \\
\text { transcription }\end{array}$ & $\begin{array}{l}\text { Relevance and Potential roles in } X \text {. laevis } \\
\text { whole body dehydration }\end{array}$ \\
\hline Sirt1 & Liver, Lung & $\begin{array}{l}\rightarrow \text { Activation of antioxidant defenses } \\
\text { through deacetylation of FoxO1 } \\
\rightarrow \text { FoxO1 acetylation promotes inhibitory } \\
\text { FoxO1 phosphorylation } \\
\rightarrow \text { FoxO1 deacetylation promotes DNA- } \\
\text { binding activity } \\
\rightarrow \text { Results in increased SOD2, decreased } \\
\text { ROS and anti-apoptosis }\end{array}$ \\
\hline Sirt2 & $\begin{array}{l}\text { Skeletal muscle, } \\
\text { Lung }\end{array}$ & $\begin{array}{l}\rightarrow \text { Activation of antioxidant signaling } \\
\text { through the elevation of both reduced } \\
\text { (GSH) and oxidized (GSSG) glutathione } \\
\text { levels } \\
\rightarrow \text { Also facilitates a high antioxidant } \\
\text { capacity by maintaining a higher } \\
\text { GSH/GSSG ratio }\end{array}$ \\
\hline Sirt3 & Skeletal muscle & $\begin{array}{l}\rightarrow \text { Activation of antioxidant defenses } \\
\text { through deacetylation and activation of } \\
\text { FoxO3 } \\
\rightarrow \text { Activation of antioxidant defenses by } \\
\text { the upregulation of both SOD2 and catalase }\end{array}$ \\
\hline Sirt4 & Liver & $\begin{array}{l}\rightarrow \text { Role in the liver is to suppress fatty acid } \\
\text { oxidation }\end{array}$ \\
\hline Sirt5 & - & $\begin{array}{l}\rightarrow \text { Hepatocyte-specific role that promotes } \\
\text { the urea cycle by deacetylating and } \\
\text { activating CPS } 1\end{array}$ \\
\hline Sirt6 & Lung & $\begin{array}{l}\rightarrow \text { Activation of antioxidant defenses by } \\
\text { direct interaction with Nrf } 2 \\
\rightarrow \text { Deacetylates Ac-H3K56 } \\
\rightarrow \text { Facilitates the expression of HO-1 } \\
\rightarrow \text { Suppresses apoptosis and necrosis }\end{array}$ \\
\hline Sirt7 & Lung & $\begin{array}{l}\rightarrow \text { Activation prevents a fibrotic phenotype } \\
\text { by suppressing genes (collagen, } \alpha \text {-SMA, } \\
\text { and Smad3 expression) characteristic of } \\
\text { pulmonary fibrosis } \\
\rightarrow \text { Sirt } 7 \text { signaling may facilitate a } \\
\text { cytoprotective pulmonary response in } \\
\text { dehydration }\end{array}$ \\
\hline
\end{tabular}




\subsection{References}

Balinsky, J.B., Choritz, E.L., Coe, C.G., van der Schans, G.S., 1967. Amino acid metabolism and urea synthesis in naturally aestivating Xenopus laevis. Comp. Biochem. Physiol. 22, 59-68.

Balinsky, J.B., Cragg, M.M., Baldwin, E., 1961. The adaptation of amphibian waste nitrogen excretion to dehydration. Comp. Biochem. Physiol. 3, 236-244.

Chen, Y., Zhang, J., Lin, Y., Lei, Q., Guan, K.L., Zhao, S., Xiong, Y., 2011. Tumour suppressor SIRT3 deacetylates and activates manganese superoxide dismutase to scavenge ROS. EMBO Rep. 12, 534-541.

Childers, C.L., Storey, K.B., 2016. Post-translational Regulation of Hexokinase Function and Protein Stability in the Aestivating Frog Xenopus laevis. Protein J. 35, 61-71.

Daitoku, H., Hatta, M., Matsuzaki, H., Aratani, S., Ohshima, T., Miyagishi, M., Nakajima, T., Fukamizu, A., 2004. Silent information regulator 2 potentiates Foxo1mediated transcription through its deacetylase activity. Proc. Natl. Acad. Sci. 101, $10042-10047$.

Dawson, N.J., Biggar, Y., Malik, A.I., Storey, K.B., 2018. Increased transcript levels and kinetic function of pyruvate kinase during severe dehydration in aestivating African clawed frogs, Xenopus laevis. Comp. Biochem. Physiol. Part - B Biochem. Mol. Biol.

Eaton, S.L., Roche, S.L., Llavero Hurtado, M., Oldknow, K.J., Farquharson, C., Gillingwater, T.H., Wishart, T.M., 2013. Total Protein Analysis as a Reliable Loading Control for Quantitative Fluorescent Western Blotting. PLoS One 8, e72457.

Hori, Y.S., Kuno, A., Hosoda, R., Horio, Y., 2013. Regulation of FOXOs and p53 by SIRT1 Modulators under Oxidative Stress. PLoS One 8, e73875.

Jacobs, K.M., Pennington, J.D., Bisht, K.S., Aykin-Burns, N., Kim, H.S., Mishra, M., Sun, L., Nguyen, P., Ahn, B.H., Leclerc, J., Deng, C.X., Spitz, D.R., Gius, D., 2008. SIRT3 interacts with the daf-16 homolog FOXO3a in the mitochondria, as well as increases FOXO3a dependent gene expression. Int. J. Biol. Sci. 4, 291-299.

Jing, E., Emanuelli, B., Hirschey, M.D., Boucher, J., Lee, K.Y., Lombard, D., Verdin, E.M., Kahn, C.R., 2011. Sirtuin-3 (Sirt3) regulates skeletal muscle metabolism and insulin signaling via altered mitochondrial oxidation and reactive oxygen species production. Proc. Natl. Acad. Sci. 108, 14608-14613.

Katzenback, B.A., Dawson, N.J., Storey, K.B., 2014. Purification and characterization of a urea sensitive lactate dehydrogenase from the liver of the African clawed frog, Xenopus laevis. J. Comp. Physiol. B Biochem. Syst. Environ. Physiol. 184, 601611. 
Liu, G., Park, S.-H., Imbesi, M., Nathan, W.J., Zou, X., Zhu, Y., Jiang, H., Parisiadou, L., Gius, D., 2017. Loss of NAD-Dependent Protein Deacetylase Sirtuin-2 Alters Mitochondrial Protein Acetylation and Dysregulates Mitophagy. Antioxid. Redox Signal. 26, 849-863.

Malik, A.I., Storey, K.B., 2011. Transcriptional regulation of antioxidant enzymes by FoxO1 under dehydration stress. Gene 485, 114-119.

Malik, A.I., Storey, K.B., 2009. Activation of extracellular signal-regulated kinases during dehydration in the African clawed frog, Xenopus laevis. J. Exp. Biol. 212, 2595-2603.

Matsuzaki, H., Daitoku, H., Hatta, M., Aoyama, H., Yoshimochi, K., Fukamizu, A., 2005. Acetylation of Foxo1 alters its DNA-binding ability and sensitivity to phosphorylation. Proc. Natl. Acad. Sci. 102, 11278-11283.

Morris, K.C., Lin, H.W., Thompson, J.W., Perez-Pinzon, M.A., 2011. Pathways for ischemic cytoprotection: Role of sirtuins in caloric restriction, resveratrol, and ischemic preconditioning. J. Cereb. Blood Flow Metab. 31, 1003-1019.

Nakagawa, T., Lomb, D.J., Haigis, M.C., Guarente, L., 2009. SIRT5 Deacetylates Carbamoyl Phosphate Synthetase 1 and Regulates the Urea Cycle. Cell 137, 560570.

Nasrin, N., Wu, X., Fortier, E., Feng, Y., Baré, O.C., Chen, S., Ren, X., Wu, Z., Streeper, R.S., Bordone, L., 2010. SIRT4 regulates fatty acid oxidation and mitochondrial gene expression in liver and muscle cells. J. Biol. Chem. 285, 31995-32002.

Pan, H., Guan, D., Liu, X., Li, J., Wang, L., Wu, J., Zhou, J., Zhang, W., Ren, R., Zhang, W., Li, Y., Yang, J., Hao, Y., Yuan, T., Yuan, G., Wang, H., Ju, Z., Mao, Z., Li, J., Qu, J., Tang, F., Liu, G.H., 2016. SIRT6 safeguards human mesenchymal stem cells from oxidative stress by coactivating NRF2. Cell Res. 26, 190-205.

Pantazi, E., Zaouali, M.A., Bejaoui, M., Folch-Puy, E., Abdennebi, H. Ben, RosellóCatafau, J., 2013. Role of sirtuins in ischemia-reperfusion injury. World J. Gastroenterol. 19, 7594-7602.

Qiu, X., Brown, K., Hirschey, M.D., Verdin, E., Chen, D., 2010. Calorie restriction reduces oxidative stress by SIRT3-mediated SOD2 activation. Cell Metab. 12, 662667.

Someya, S., Yu, W., Hallows, W.C., Xu, J., Vann, J.M., Leeuwenburgh, C., Tanokura, M., Denu, J.M., Prolla, T.A., 2010. Sirt3 mediates reduction of oxidative damage and prevention of age-related hearing loss under Caloric Restriction. Cell 143, 802812.

Storey, K.B., Storey, J.M., 2012. Aestivation: signaling and hypometabolism. J. Exp. Biol. 215, 1425-33.

Sundaresan, N.R., Gupta, M., Kim, G., Rajamohan, S.B., Isbatan, A., Gupta, M.P., 2009. 
Sirt3 blocks the cardiac hypertrophic response by augmenting Foxo3a-dependent antioxidant defense mechanisms in mice. J. Clin. Invest. 119, 2758-2771.

Vannini, A., Volpari, C., Filocamo, G., Casavola, E.C., Brunetti, M., Renzoni, D., Chakravarty, P., Paolini, C., De Francesco, R., Gallinari, P., Steinkuhler, C., Di Marco, S., 2004. Crystal structure of a eukaryotic zinc-dependent histone deacetylase, human HDAC8, complexed with a hydroxamic acid inhibitor. Proc. Natl. Acad. Sci. 101, 15064-15069.

Wang, S.J., Zhao, X.H., Chen, W., Bo, N., Wang, X.J., Chi, Z.F., Wu, W., 2015. Sirtuin 1 activation enhances the PGC-1a/mitochondrial antioxidant system pathway in status epilepticus. Mol. Med. Rep. 11, 521-526.

Wang, X.X., Wang, X.L., Tong, M.M., Gan, L., Chen, H., Wu, S.S., Chen, J.X., Li, R.L., Wu, Y., Zhang, H.Y., Zhu, Y., Li, Y.X., He, J.H., Wang, M., Jiang, W., 2016. SIRT6 protects cardiomyocytes against ischemia/reperfusion injury by augmenting FoxO3a-dependent antioxidant defense mechanisms. Basic Res. Cardiol. 111, 1-19.

Welinder, C., Ekblad, L., 2011. Coomassie staining as loading control in Western blot analysis. J. Proteome Res. 10, 1416-1419.

Wyman, A.E., Noor, Z., Fishelevich, R., Lockatell, V., Shah, N.G., Todd, N.W., Atamas, S.P., 2017. Sirtuin 7 is decreased in pulmonary fibrosis and regulates the fibrotic phenotype of lung fibroblasts. Am. J. Physiol. - Lung Cell. Mol. Physiol. 312, L945-L958.

Zhang, J., Storey, K.B., 2016. RBioplot: an easy-to-use R pipeline for automated statistical analysis and data visualization in molecular biology and biochemistry. PeerJ 4, e2436.

Zhang, S., Jiang, S., Wang, H., Di, W., Deng, C., Jin, Z., Yi, W., Xiao, X., Nie, Y., Yang, Y., 2018. SIRT6 protects against hepatic ischemia/reperfusion injury by inhibiting apoptosis and autophagy related cell death. Free Radic. Biol. Med. 115, 18-30.

Zhao, S., Xu, W., Jiang, W., Yu, W., Lin, Y., Zhang, T., Yao, J., Zhou, L., Zeng, Y., Li, H., Li, Y., Shi, J., An, W., Hancock, S.M., He, F., Qin, L., Chin, J., Yang, P., Chen, X., Lei, Q., Xiong, Y., Guan, K.L., 2010. Regulation of cellular metabolism by protein lysine acetylation. Science 327, 1000-1004.

Zitka, O., Skalickova, S., Gumulec, J., Masarik, M., Adam, V., Hubalek, J., Trnkova, L., Kruseova, J., Eckschlager, T., Kizek, R., 2012. Redox status expressed as GSH:GSSG ratio as a marker for oxidative stress in paediatric tumour patients. Oncol. Lett. 4, 1247-1253. 


\section{CHAPTER 3}

\section{The role of Sirtuin-regulated Antioxidant Signaling in Response to Dehydration}




\subsection{Introduction}

\section{$\underline{3.1 .1 \text { Sirts and antioxidant regulation }}$}

In Chapter 2, it was revealed that gene expression of select Sirt genes and Sirt deacetylase activity was upregulated in multiple $X$. laevis tissues in response to wholebody dehydration. The genes that were differentially expressed in select tissues included sirt1, sirt2, and sirt6. Furthermore, an antioxidant enzyme (SOD2) target residue for deacetylation by SIRT3 showed decreased acetylation levels in select tissues during dehydration, which is suggestive of increased antioxidant activity. As discussed in Chapter 2, SIRT1, SIRT2, SIRT3, and SIRT6 are known to have roles in regulating antioxidant signaling pathways. The present chapter builds on the previous chapter's results by exploring Sirt-controlled antioxidant signaling pathways. The focus in this chapter is on the lung and brain, which provided a comparative analysis of antioxidant signaling in a tissue that demonstrated the strongest activation of Sirt signaling (lung), and a tissue where dehydration did not exert any observed Sirt regulation (brain). A finding that antioxidant signaling increases in the lungs, but not the brain of $X$. laevis, would provide evidence that supports the notion that increased blood circulation to the brain (Hillman and Sommerfeldt, 1981) is sufficiently neuroprotective so that brain does not warrant increased antioxidant signaling during dehydration.

\section{$\underline{\text { 3.1.2 Sirt regulation of PGC-1 } \alpha \text { and FoxO transcription factors }}$}

The expression of antioxidant genes by Sirt proteins is complex and implicates variables beyond gene, protein, and global Sirt activity levels. As a result, there are other targets related to the Sirt-mediated antioxidant response that warrant investigation in $X$. laevis dehydration. In one study, SIRT1 regulation of antioxidant genes has been found to 
be dependent PGC-1 $\alpha$ and FoxO3. In a culture of endothelial cells, overexpression of SIRT1 will result in an increase in antioxidant genes such as SOD2/MnSOD and catalase (Olmos et al., 2013). However, when either PGC-1 $\alpha$ or FoxO3 were knocked down, SIRT1 overexpression no longer resulted in an induction of antioxidant gene expression (Olmos et al., 2013). In addition to the expression of antioxidant genes, it was demonstrated that SIRT1 has a role in the expression of pgc-1 $\alpha$ and foxo3 genes (Olmos et al., 2013). The role of SIRT1 in the regulation of these genes was further confirmed by chromatin immunoprecipitation which demonstrated that SIRT1 also directly binds to the promoter regions of sod2, catalase, pgc-1 $\alpha$, and foxo3 (Olmos et al., 2013). Importantly, the authors also demonstrated that SIRT1-mediated upregulation of $p g c-1 \alpha$, foxo3, and antioxidant genes also increased the antioxidant capacity of the cells. As a result of these past findings, the present study investigated the effects of $X$. laevis dehydration on the SIRT1/PGC-1 $\alpha /$ FoxO3 pathway and its downstream antioxidant targets, SOD2 and catalase in the lung and brain.

As discussed in Chapter 2, there are multiple interactions between Sirt proteins and FoxO transcription factors that have been previously demonstrated. In addition to the role of SIRT1 in regulating FoxO3 and PGC-1 $\alpha$, SIRT1 also interacts with FoxO1. In particular, one of the roles of SIRT1 is to remove inhibitory acetylation on FoxO1 that attenuates DNA binding and promotes inhibitory protein phosphorylation (Daitoku et al., 2004; Matsuzaki et al., 2005). In doing so, FoxO1 may promote the transcription of its target genes, such as the antioxidant enzymes catalase and SOD2 (Awad et al., 2014; Daitoku et al., 2004). In addition to SIRT1, there are other SIRT proteins that facilitate FoxO and antioxidant signaling. Studies have shown that SIRT2 plays a role in 
maintaining the levels of GSH, an important free radical scavenger in the cell (Liu et al., 2017). Like SIRT1, the SIRT3 protein also interacts with FoxO3 by deacetylating the FoxO3 transcription factor, which resulted in this transcription factor's nuclear localization, transcription of antioxidant genes, and increased antioxidant capacity (Jacobs et al., 2008; Sundaresan et al., 2009). For these reasons, this chapter characterized the dehydration-induced effects on FoxO1 and FoxO3 in the lung and brain of X. laevis.

\section{$\underline{\text { 3.1.3 Antioxidant enzymes }}$}

The SIRT, PGC-1 $\alpha$ and FoxO proteins have important roles in antioxidant signaling, such as the regulation of sod2 and catalase antioxidant genes. These antioxidant enzymes assist with the reactive oxygen species that can be expected to accumulate during periods of impaired oxygen transport caused by periods of dehydration and rehydration. The Mn-dependent SOD2 enzyme functions by catalyzing the conversion of superoxides into $\mathrm{H}_{2} \mathrm{O}_{2}$, and catalase facilitates the breakdown of $\mathrm{H}_{2} \mathrm{O}_{2}$ into water and $\mathrm{O}_{2}$ (Chelikani et al., 2004; Kawamata and Manfredi, 2010). In addition, $\mathrm{Cu} / \mathrm{Zn}$-dependent SOD1 and heme oxygenase-1 (HO-1) are antioxidant enzymes that are known to be regulated by PGC-1 $\alpha$ and FoxO proteins, respectively (Kang et al., 2014; Kawamata and Manfredi, 2010; Tsang et al., 2014). The dismutase function of SOD1 is similar to that of SOD2, except the former is localized in the multiple subcellular compartments ranging from the mitochondria, nucleus, and cytosol (Kawamata and Manfredi, 2010; Tsang et al., 2014), whereas SOD2 is located exclusively in the mitochondria (Karnati et al., 2013). The HO-1 is the stress-inducible isoform (the other constitutively expressed isoform being $\mathrm{HO}-2$ ) of an enzyme that catalyzes the breakdown 
of heme into biliverdin, iron, and CO (Ryter, 2006). In the pathway of heme metabolism, biliverdin is further processed by biliverdin reductase into bilirubin, which has been shown to protect endothelial cells, vascular smooth muscle cells, and cardiomyocytes from cytotoxicity induced by $\mathrm{H}_{2} \mathrm{O}_{2}$ and/or enzymatically produced reactive oxygen species (Clark et al., 2000; Motterlini et al., 1996; Wu et al., 1991). One group has suggested that biliverdin reductase acts as a regenerator of cellular antioxidant capacity by reducing the biliverdin formed from reoxidized bilirubin. This group demonstrated that a knockdown of biliverdin reductase increased cellular levels of reactive oxygen species and promoted apoptotic cell death in HeLa and primary neuronal cultures (Baranano et al., 2002). In addition to assessing PGC-1 $\alpha$, FoxO1 and FoxO3 targets, this chapter also investigates how SOD1, SOD2, catalase, and HO-1 are regulated in the lung and brain of $X$. laevis during dehydration.

\subsubsection{Chapter objective}

The present chapter builds on the findings in Chapter 2 by assessing the relative protein levels of SIRT proteins (SIRT1, SIRT2, SIRT3, and SIRT6) known to be important in antioxidant signaling. This comparison is performed in two tissues in $X$. laevis under control, medium dehydration, and high dehydration conditions; the lungs and the brain, which showed contrastingly strong and minor SIRT signaling in Chapter 2. Tissue protein extracts were assessed via immunoblotting to measure the relative protein levels of SIRT targets. Secondly, the transcriptional regulators of antioxidant signaling (PGC-1 $\alpha$, FoxO1, and FoxO3) that are known to interact with SIRT proteins are assessed in these two tissues. These targets will be assessed at the mRNA level with RTqPCR and at total protein level with immunoblotting. Since SIRT-targeted FoxO protein 
acetylation is known to promote inhibitory phosphorylation of FoxO transcription factors that prevents its DNA binding activity, immunoblotting was also used to assess the relative levels of serine-phosphorylated and threonine-phosphorylated FoxO transcription factors in $X$. laevis lung and brain protein extracts. Thirdly, the downstream antioxidant enzymes SOD1, SOD2, catalase, and HO-1 that are known to be under the transcriptional control of PGC-1 $\alpha$, FoxO1, and FoxO3 were assessed in both tissues under control and dehydration conditions. These comparative analyses were performed with RT-qPCR to measure relative transcript levels, and immunoblotting to assess relative protein and relative phospho-protein levels. Lastly, an in vitro antioxidant capacity assay was used to quantify the relative capacity in 6 tissues, in which tissue lysates from animals under different levels of dehydration could prevent artificially-generated oxidation of an assay substrate. 


\subsection{Materials and Methods}

\subsubsection{Animals}

All animal experiments were conducted s described in Chapter 2.

\section{$\underline{3.2 .2 \text { RNA extraction }}$}

Isolation of RNA from $X$. laevis tissues was performed as described in Chapter 2.

\section{$\underline{3.2 .3 \text { cDNA synthesis }}$}

Synthesis of cDNA samples was performed as described in Chapter 2.

\subsubsection{Primer design}

Primers were designed for RT-qPCR as previously described in Chapter 2.

Primers were designed for the relative quantification of pgc-1 $\alpha$, foxol, foxo 3 , catalase, ho-1, sod1, and sod2. Details for each primer (sequence, efficiencies) and the X. laevis $\mathrm{NCBI}$ accession numbers for the gene sequences used for primer design can be found in the Appendix C.

\subsubsection{RT-qPCR}

Relative gene quantification was performed with RT-qPCR as previously described in Chapter 2.

\subsubsection{Protein extraction}

Total protein extraction was performed as previously described in Chapter 2 . 


\subsubsection{Western immunoblotting}

Immunoblotting was performed as previously described in Chapter $\mathbf{2}$ with minor modifications. Antibodies were used to probe for SIRT1, SIRT2, SIRT3, SIRT6, PGC1a, FoxO1, p-FoxO1 (Ser256), p-FoxO1 (Ser319), FoxO3, p-FoxO3 (Ser253), Catalase, HO-1, SOD1, and SOD2. Specific experimental conditions that were used for each of the antibodies can be found in the Appendices $\mathbf{H}$ \& $\mathbf{I}$.

\subsubsection{Measurement of Antioxidant Capacity}

Cell lysates of $X$. laevis tissues under control, medium, and high dehydration conditions were used to measure relative changes in antioxidant capacity. All reagents for this assay were obtained from the Cayman Chemical Company (Ann Arbor, MI). Cell lysates were all pre-diluted at least 1:5 v:v in Antioxidant Assay Buffer (Cayman Chemicals Cat. \# 709002). Assay reactions were prepared by combining $10 \mu 1$ of diluted cell lysate, $10 \mu 1$ of suspended metmyoglobin (Cayman Chemicals Cat. \#10004875) and $150 \mu 1$ of suspended chromogen (Cayman Chemicals Cat. \#10004873). In order to initiate the assay reaction, $40 \mu 1$ of $441 \mu \mathrm{M}$ hydrogen peroxide (Cayman Chemicals Cat. $\# 10004877)$ was added to the reaction volume. Reactions were covered, shaken for 5-10 minutes at room temperature, and assayed in a microplate at $405 \mathrm{~nm}$ using a plate reader. The dynamic range of this assay was determined for each tissue using a range of protein amounts. Quantification of relative antioxidant capacity was performed with the protein amount that appears in the middle of the dynamic range in order so that the quantification is performed within the limit of detection, but also not at a point of signal oversaturation. A linear regression of a Trolox (Cayman Chemicals Cat. \# 10004876) standard curve generated with this assay was used to calculate relative antioxidant concentrations from 
the cell lysates. Relative antioxidant capacity of tissues under control, medium, and high dehydration conditions was calculated by averaging calculated antioxidant concentrations for each experimental condition, and normalizing experimental values to the control, which was set to 1 .

This antioxidant capacity assay is designed to represent the summed antioxidant capacity of a sample made up by a number of different antioxidant molecules. The assay is designed to quantify the sample's ability to prevent the oxidation of ABTS (2,2'Azino-di-[3-ethylbenzthiazoline sulphonate]) by the metmyoglobin present in the assay reaction. While many antioxidants such as glutathione, vitamins $\mathrm{C}$ and $\mathrm{E}, \mathrm{BSA}$, and uric acid are able to prevent ABTS oxidation by metmyoglobin, other antioxidants such as bilirubin are not. Thus, it is important to note that the results from this assay are not an exhaustive representation of antioxidant capacity in the cell lysate samples. 


\subsection{Results}

\subsubsection{Relative protein levels of SIRT1, SIRT2, SIRT3 and SIRT6}

Previous studies have shown that SIRT1, SIRT2, SIRT3 and SIRT6 proteins play roles in antioxidant signaling (discussed in Chapter 2). Using immunoblotting, relative protein levels of these targets were assessed in lung and brain of $X$. laevis exposed to control, medium dehydration, and high dehydration conditions. In contrast to lung sirt1 mRNA transcript levels that increased in response to high dehydration (Chapter 2), lung SIRT1 protein levels decreased to $0.47 \pm 0.06$ and $0.40 \pm 0.14$ under medium and high dehydration conditions, respectively (Figure 3.1). Compared to controls, lung SIRT3 protein levels were found to increase by $3.05 \pm 0.32$ fold in medium dehydration, and $5.43 \pm 0.78$ fold in high dehydration (Figure 3.1). However, despite observed increases in lung sirt2 and sirt6 transcript levels (Chapter 2), there were no dehydration-induced changes to relative SIRT2 and SIRT6 protein content in the lungs of $X$. laevis (Figure 3.1). Furthermore, there were no changes to protein levels of SIRT1, SIRT2, SIRT3, or SIRT6 in X. laevis brain under either medium or high dehydration conditions (Figure 3.2).

\subsubsection{Regulation of lung and brain PGC-1 $\alpha$ mRNA and protein levels}

In the lung and brain, RT-qPCR and immunoblotting were used to assess the relative levels of PGC-1 $\alpha$ mRNA and protein levels. In the lung, pgcl $\alpha$ mRNA increased by $8.22 \pm 2.23$ fold under high dehydration conditions (Figure 3.3A). Consistently, PGC$1 \alpha$ protein levels also increased in lungs under both medium and high dehydration conditions, by $2.54 \pm 0.20$ and $1.95 \pm 0.23$ fold, respectively (Figure 3.3B). In the brain, a $1.56 \pm 0.16$ fold increase in pgcla mRNA levels over controls occurred in response to 
medium dehydration, but no change was observed between control and high dehydration conditions (Figure 3.3A). Protein levels of PGC-1 $\alpha$ in the brain did not change in response to either dehydration stress (Figure 3.3B).

\subsubsection{Regulation of FoxO1 in lung and brain}

FoxO1 from the lung and brain of dehydrating $X$. laevis was investigated, analyzing FoxO1 at transcript, protein, and protein phosphorylation levels. A sequence alignment was performed to insure that the phosphorylated residues to be assessed were conserved between $X$. laevis and the respective mammalian targets for which the antibodies were designed (Figure 3.4). The alignment showed that the phospho-residues (and the surrounding up- and down-stream amino acids) were conserved across the four species ( $X$. laevis, human, rat, mouse). The sequence analysis confirmed that the human FoxO1 Ser256 and Ser319 residues appeared as Ser245 and Ser308, respectively, in the X. laevis protein (Figure 3.4). In the lungs, exposure to high dehydration induced a $2.67 \pm 0.34$ fold increase in foxol transcript levels, whereas dehydration did not change relative foxo1 mRNA levels in brain (Figure 3.5A).

Relative protein abundance quantified with immunoblots revealed a $2.42 \pm 0.11$ fold increase in FoxO1 protein levels in the lungs, but no differences were observed between control and medium dehydration conditions (Figure 3.5B). Relative FoxO1 protein levels in the brain did not change with dehydration exposure.

Immunoblotting was also used to quantify the relative phosphorylation states of FoxO1 proteins in lung and brain. In the lungs, the relative phosphorylation of the serine 245 residue of FoxO1 (p-FoxO1 S245) decreased significantly under medium and high 
dehydration conditions to $0.71 \pm 0.11$ and $0.27 \pm 0.01$ of control levels, respectively (Figure 3.5B). Likewise, p-FoxO1 S308 content also decreased in the lungs under high dehydration exposure to $0.66 \pm 0.07$ of control levels, but values were not affected in the medium dehydration condition (Figure 3.5B). In brain, however, FoxO1 protein levels and relative phosphorylation of p-FoxO1 S245 and p-FoxO1 S308 were unchanged in response to dehydration (Figure 3.5B).

\subsubsection{Regulation of FoxO3 in lung and brain}

Similar to the results for FoxO1, FoxO3 also responded to dehydration in $X$. laevis lungs. High dehydration conditions led to a $5.16 \pm 0.40$ fold increase in foxo3 transcript levels, when compared to control conditions (Figure 3.6A). In the brain, foxo3 transcript levels also increased by $1.61 \pm 0.09$ fold under medium dehydration conditions, but were not different from controls under high dehydration (Figure 3.6A).

Relative FoxO3 protein and phosphorylated FoxO3 protein levels were assessed with immunoblotting in $X$. laevis lungs and brains. Lung FoxO3 protein levels increased under both medium and high dehydration conditions, by $10.74 \pm 1.04$ and $4.27 \pm 0.72$ fold respectively, as compared to control levels (Figure 3.6B). In contrast, FoxO3 protein abundance in the brain was not sensitive to dehydration. Relative phosphorylation at the FoxO3 Ser238 residue of FoxO3 in X. laevis showed no dehydration-induced changes in either lung or brain (Figure 3.6B).

\subsubsection{Regulation of catalase and HO-1}

Expression of catalase and HO-1 transcripts is regulated by SIRT and PGC-1 $\alpha$ proteins in addition to FoxO transcription factors. The relative transcript and protein levels of these two proteins were also assessed in the lungs and brain of dehydrating $X$. 
laevis. In the lungs, cat1 transcript levels did not change with high dehydration exposure, but ho- 1 transcripts increased $2.77 \pm 0.43$ fold as compared to controls (Figure 3.7A). In contrast to mRNA levels, catalase protein levels in the lungs increased by $1.95 \pm 0.15$ fold with high dehydration exposure whereas HO-1 protein in lung rose by $2.32 \pm 0.27$ and $3.40 \pm 0.14$ fold under medium and high dehydration, respectively (Figure 3.7B).

In brain, the transcript levels of both cat- 1 and ho- 1 showed an increasing trend but the data were not significant (Figure 3.7A). Dehydration also had no effect on catalase or HO-1 protein levels in brain (Figure 3.7B).

\subsubsection{Assessment of antioxidant capacity}

An antioxidant capacity assay was used to quantify the relative ability of tissue samples to prevent oxidation of ABTS by metmyoglobin. In order to quantify the relative antioxidant capacities of tissue samples, assays were performed for cell lysates of six tissues obtained from control, medium dehydration, and high dehydration conditions. A significant decrease in antioxidant capacity was observed in lungs under high dehydration exposure, levels falling to $0.65 \pm 0.03$ of control antioxidant capacity (Figure 3.8 ). In heart, a modest, albeit significant, decrease in antioxidant capacity also occurred in the high dehydration condition, resulting in a decrease to $0.96 \pm 0.008$ of control values (Figure 3.8). However, antioxidant capacity was unaffected by dehydration in liver, skeletal muscle, brain and kidney.

\subsubsection{Regulation of SOD1 and SOD2}

SOD1 and SOD2 were also assessed at the mRNA and protein levels in lung and brain samples of dehydration-exposed $X$. laevis. In the lungs, sod1 transcript levels increased by $1.40 \pm 0.12$ fold over control levels under high dehydration exposure (Figure 
3.9A). However, sod2 lung transcript levels were not significantly different between control and dehydrated conditions. Furthermore, neither $\operatorname{sod} 1$ or $\operatorname{sod} 2$ transcript levels in brain were sensitive to dehydration exposure. Immunoblotting was used to assess SOD1 and SOD2 protein levels in lungs and brains, but protein abundance did not change in response to dehydration (Figure 3.9B). 


\subsection{Discussion}

In this chapter, the downstream antioxidant pathway that is controlled by specific Sirt proteins was assessed in the lung and brain of $X$. laevis under control and dehydrated states. A comparison between the lung and brain provided evidence that the molecular responses to dehydration are tissue-specific. The lungs of $X$. laevis were specifically chosen because they have an obvious role of facilitating gas exchange, a role that may change substantially during dehydration when cutaneous gas exchange is impaired/halted and when lung gas exchange could be affected by reduced hydration of lung tissue itself with an overall effect of impairing oxygen delivery to tissues in the body (Hillman, 1978). Although the skin may account for up to $30 \%$ of $\mathrm{O}_{2}$ uptake in X. laevis, the lungs are primarily relied on for breathing, particularly when $X$. laevis is not undergoing a dive (Emilio and Shelton, 1974). Furthermore, Chapter 2 revealed that the lungs showed high Sirt signaling in response to dehydration exposure, as evidenced by increased mRNA levels of select sirt genes, increased global Sirt activity, and decreased acetylation of known Sirt antioxidant protein substrates. This contrasts with the $X$. laevis brain, where previous studies have shown an increase in blood circulation in response to dehydration (Hillman and Sommerfeldt, 1981), and where Sirt signaling appeared to be nonresponsive. Together, the evidence suggests that a compensatory increase in blood circulation to the brain may mitigate the need for antioxidant signaling, whereas these signaling pathways are required and activated in the lungs.

\subsubsection{Sirt proteins in antioxidant signaling}

Relative protein levels of individual Sirt proteins (SIRT1, SIRT2, SIRT3, and SIRT6) that are known to regulate antioxidant signaling were assessed in the lung and 
brain of $X$. laevis under dehydration conditions. A significant increase in SIRT3 protein levels was observed in the lungs of frogs under medium and high dehydration conditions (Figure 3.1). Although results from the previous chapter demonstrated that lung sirt3 mRNA levels were non-responsive to $X$. laevis dehydration, the increase in lung SIRT3 protein paralleled the increase in global Sirt deacetylase activity and a corresponding decrease in lung of Ac-SOD2 (K70) content, a SIRT3-specific substrate (Chen et al., 2011; Qiu et al., 2010). In conjunction with Chapter 2, this suggests that activation of antioxidant signaling in the lungs of dehydrating $X$. laevis involves the SIRT3-mediated deacetylation of SOD2 (Figure 3.10). These changes in SIRT3 protein and Ac-SOD2 content implicate dehydration-induced regulation at the protein and post-translational modification levels, but not the transcript level. These results also demonstrate that the control of signaling during dehydration is dynamic, and changes may not be occurring at all levels of gene and protein regulation.

A disconnect between mRNA and protein levels was seen for SIRT2 and SIRT6 in the lungs of dehydrated $X$. laevis. Data in Chapter 2 showed a significant increase in lung sirt2 and sirt6 mRNA levels, but protein levels reported in this chapter showed no change in SIRT2 and SIRT6 protein levels in lungs when X. laevis were dehydrated (Figure 3.1). The roles of SIRT2 and SIRT6 in antioxidant signaling include maintaining levels of GSH, and activation of FoxO3 transcription, respectively (Liu et al., 2017; Wang et al., 2016). Although the results from the present chapter demonstrate that SIRT2 and SIRT6 proteins are not prioritized by lung for upregulation, as was the case for SIRT3, it cannot be concluded that they are not significant factors in the response to dehydration by $X$. laevis. Only assessments of global Sirt deacetylase activity were 
performed on lung samples, and so the present studies cannot comment on the contributions by each individual SIRT protein to total activity. As previously mentioned, SIRT2 has been shown to sustain the antioxidant system by maintaining the GSH/GSSH ratio (Liu et al., 2017), although the mechanism by which this occurs has not yet been fully elucidated. Importantly, a previous study on X. laevis has demonstrated that there may be active metabolic roles for glutathione in the lungs during dehydration, as evidenced by increased protein amounts of glutathione-S-transferases, which are important enzymes for detoxification (Malik and Storey, 2009). Hence, the present results suggest that sirt2 signaling may contribute to the maintenance of GSH/GSSH ratios (Figure 3.10). However, this proposal would benefit further from investigation of the proteins that contribute to maintaining GSH/GSSH ratios, such as glutathione reductase.

In addition to SIRT2 and SIRT6, SIRT1 protein levels in the X. laevis lungs similarly did not correlate with measured sirt 1 mRNA levels. Whereas sirt1 transcript levels increased during dehydration, SIRT1 protein levels actually decreased in response to medium and high levels of dehydration (Figure 3.1). In Chapter 2, it was expected that lung SIRT1 protein levels would either be maintained or upregulated due to an increase in sirt1 mRNA during dehydration, potentially supporting a role for SIRT1 in activating antioxidant signaling via deacetylation of FoxO transcription factors leading to upregulated expression of antioxidant genes (Daitoku et al., 2004; Matsuzaki et al., 2005). However, other studies on cell lines have shown that hypoxia results in the suppression of SIRT1 expression (Leiser and Kaeberlein, 2010) and that SIRT1 is capable of deacetylating and inactivating hypoxia inducible factor-1 $\alpha$ (HIF-1 $\alpha)$, a transcription factor responsible for activating hypoxia response genes (Leiser and 
Kaeberlein, 2010). Given that $X$. laevis increases sirt1 mRNA levels and global Sirt activity in the lungs, it can be postulated that the observed decrease in SIRT1 protein levels may be facilitated by other modes of regulation, such as post-transcriptional regulation of sirt1 expression by non-coding RNAs. This may also apply to SIRT3, where protein levels increased during dehydration, but mRNA levels did not - again implicating an intervening posttranscriptional regulation. These results from the Sirt protein analyses demonstrate that regulation of these deacetylases is complex, and further downstream investigation of Sirt targets is necessary. Indeed, as noted previously, one of the modes by which $X$. laevis may be increasing antioxidant activity in the lungs may be through upregulation of SIRT3 protein and deacetylation of SOD2 (Figure 3.10).

\subsubsection{PGC-1 $\alpha$ / FoxO1 / FoxO3 signaling}

One mechanism by which Sirt signaling initiates antioxidant responses is through the interaction with PGC-1 $\alpha$ and the FoxO1 and FoxO3 transcription factors. In this chapter, it is demonstrated that these three targets were regulated in the lungs of dehydrated X. laevis, but not in the brain. In the lungs, relative mRNA levels of $p g c-1 \alpha$, foxo 1 , and foxo 3 were all upregulated in response to dehydration (Figures 3.3A, 3.5A and 3.6A). Corresponding to the increased mRNA levels, there were also increases in the protein levels of all three targets in the lungs (Figures 3.3B, 3.5B and 3.6B). This suggests that PGC-1 $\alpha$, FoxO1 and FoxO3 are themselves being transcribed and translated in the lungs, in contrast to the brain, where these responses were not as prominent. Again, the upregulation of these genes has been shown to be mediated by SIRT1 activity (Daitoku et al., 2004; Matsuzaki et al., 2005; Olmos et al., 2013). Furthermore, the relative amounts of p-FoxO1 S245 and S308 decreased in the lungs 
during dehydration (Figure 3.5). Since it has been shown that acetylation of FoxO1 promotes inhibitory phosphorylation of conserved serine residues (Figure 3.4), a relative decrease in phosphorylated FoxO1 suggests that this transcription factor was deacetylated during dehydration by an enzyme such as SIRT1, an interaction which has been previously demonstrated to occur in cell lines (Matsuzaki et al., 2005). Furthermore, the observed decrease in phosphorylated FoxO1 suggests that this transcription factor is being localized to the nucleus where it can induce transcription of its target genes (Matsuzaki et al., 2005). This is because the highly conserved serine residues that are targeted for phosphorylation are tightly linked with the SIRT1-controlled acetylation state and inhibitory characteristics such as cytoplasmic localization and reduced DNA binding.

FoxO3 showed a somewhat different pattern of response to dehydration stress in lungs. Although the phosphorylation state of FoxO3 proteins did not change in response to dehydration in lungs, an increase in foxo3 mRNA levels, coupled with a strong increase in FoxO3 protein levels in the lungs suggests that FoxO3 signaling is also activated during dehydration (Figure 3.6). From previous studies, it can be postulated that increases in PGC-1 $\alpha$ and FoxO3 levels are facilitated by SIRT1 (Olmos et al., 2013). Indeed, in vitro overexpression of sirt1 resulted in the upregulation of FoxO3 (Olmos et al., 2013). However, given that SIRT1 protein levels decrease in the lungs, it is clear that the regulation of the SIRT1/PGC-1 $\alpha /$ FoxO3 signaling pathway in dehydrating $X$. laevis is not as straightforward as was observed in cell lines. Despite the decrease in SIRT1 levels in the lungs of $X$. laevis, the results suggest an increase in PGC- $1 \alpha / \mathrm{FoxO} / / \mathrm{FoxO} 3$ signaling in response to dehydration, which ultimately has the potential to upregulate antioxidants downstream (Figure 3.10). 


\subsubsection{Catalase and HO-1}

Catalase and HO-1 are two downstream genes that are regulated by SIRT1/PGC$1 \alpha /$ FoxO1/FoxO3 signaling. In the lungs of dehydrated $X$. laevis, ho-1 mRNA levels increased strongly in response to dehydration although catalase mRNA did not (Figure 3.7A). However, both catalase and HO-1 protein levels increased markedly in dehydration (Figure 3.7B). The increase in catalase protein without a change in transcript levels suggests that mechanisms of post-transcriptional regulation of existing mRNA may be modulated in X. laevis dehydration. Furthermore, the nuclear factor erythroid 2-related factor (Nrf2), a dynamic transcription factor that also regulates antioxidant genes such as ho-l (Loboda et al., 2016), has been previously shown to be upregulated in lungs of $X$. laevis during dehydration (Malik and Storey, 2009). Increases in both PGC-1 $\alpha$ and Nrf2 would contribute to the observed increase in HO-1 synthesis. Overall, dehydration appeared to facilitate regulatory signaling that increased relative catalase and $\mathrm{HO}-1$ enzyme levels, responses that could provide cytoprotective effects by increasing $\mathrm{H}_{2} \mathrm{O}_{2}$ breakdown and abundance of bilirubin as a free radical scavenger (Figure 3.10).

\subsubsection{Decrease in antioxidant capacity}

In order to shed light on the state of the $X$. laevis antioxidant system during exposure to dehydration, the relative antioxidant capacity was assessed in 6 tissues of $X$. laevis from control versus dehydrated animals. The data showed that lung was unique in experiencing a strong decrease in antioxidant capacity in response to dehydration whereas the other tissues showed little (heart) or no change (brain, liver, muscle, and kidney) (Figure 3.8). These data indicating that antioxidants are depleted in the lungs in response to dehydration further underscore the importance of the SIRT-regulated 
antioxidant responses that were dehydration-activated in this tissue (Figure 3.10). The unaltered antioxidant capacity of $X$. laevis brain suggests that antioxidant activation by Sirt activity has a lesser role in the brain of $X$. laevis. This may be the case because increased blood circulation to the brain could compensate for a less effective circulatory system caused by decreased plasma volume. As a result, the cellular responses that mediate antioxidant responses, as observed in the lungs, are not required in the brain.

In the $X$. laevis heart, there was a very modest yet statistically significant decrease in antioxidant capacity in the high dehydration condition (Figure 3.8). Although FoxO transcription factors and antioxidant downstream targets were not assessed in the heart, it can be reasonable for this organ to activate stress response signaling since the whole body copes with a decrease in oxygen availability, as evidenced by an increase in the difference in arterial-venous oxygen levels (Hillman, 1978). Furthermore, it is known that $X$. laevis heart rate increases in response to dehydration, which would suggest higher metabolic demands that may contribute to the depletion of the tissue's antioxidant pool. Interestingly, results from Chapter 2 found that antioxidant signaling may be activated in the heart of $X$. laevis through SOD2 deacetylation in the high dehydration condition. Although antioxidant signaling does not seem to be as pronounced in the heart as it is in the lungs, further investigations are warranted to better understand the molecular responses that occur in the $X$. laevis heart during dehydration.

The antioxidant capacity assay utilized in the present chapter relies on the ability of samples to prevent the oxidation of ABTS, when metmyoglobin and $\mathrm{H}_{2} \mathrm{O}_{2}$ are added. Therefore, the assay is expected to capture the representative antioxidant capacity of the sample through the combined activity of multiple antioxidants, which include 
glutathione, vitamins, and proteins. However, standard curves of glutathione, vitamins C and E, and bilirubin generated by the manufacturer for this assay demonstrated that all of these compounds prevent the oxidation of ABTS at different rates. In addition to the $X$. laevis brain, the results from this assay found that exposure to dehydration did not have an effect on the relative antioxidant capacities of liver, skeletal muscle, or kidney lysates (Figure 3.8). Increases in FoxO1 signaling which include stimulation of increased SOD2 transcript and protein levels, were observed in liver of dehydrated X. laevis (Malik and Storey, 2011). Furthermore, catalase mRNA increased in liver, and catalase protein rose in both liver and skeletal muscle of dehydrated X. laevis (Malik and Storey, 2011). Since there was no observed increase in measured antioxidant capacity in the liver of $X$. laevis, a number of causal reasons can be considered: i) Dehydration-activated signaling is adequately replenishing antioxidants by FoxO-mediated antioxidant expression; ii) the increases in antioxidant response (e.g. catalase upregulation) in the liver do not significantly increase the overall antioxidant capacity, or iii) the increases in FoxOcontrolled antioxidants (SOD and bilirubin from HO-1) are not captured by the antioxidant assay measurements. Importantly, a standard curve showed that increasing bilirubin concentrations is not detected by this assay, which proposes that the effect of lung HO-1 upregulation is not accurately represented in this measurement. Therefore, although the results do suggest that high dehydration depletes of some antioxidants in the lungs, they are not an exhaustive representation of the cellular antioxidant capabilities. However, the results together do suggest that antioxidant signaling is activated during dehydration as antioxidant pools are depleted in the lungs of X. laevis (Figure 3.10).

\subsubsection{Regulation of superoxide dismutase}


Other antioxidant enzymes under the control of SIRT/PGC-1 $\alpha /$ FoxO signaling are the superoxide dismutases, SOD1 and SOD2. Despite a strong response by these upstream antioxidant regulators in the lungs of $X$. laevis, there was not a corresponding increase response in SOD1 and SOD2 expression. The lungs did demonstrate increased sod1 mRNA levels, but relative protein levels of SOD1 and SOD2 remained constant throughout dehydration exposure in lungs and brain (Figure 3.9). Although SOD1 and SOD2 protein levels did not increase, SOD activity overall may still be increasing in response to dehydration by other mechanisms such as SIRT3-mediated deacetylation of SOD2 protein, as observed in the lungs and discussed in Chapter 2. Furthermore, constant protein levels of total SOD2 in the lungs suggest that decreased Ac-SOD2 levels are a result of decreased acetylation on a SOD2 residue, rather than a decrease in total SOD2 protein levels. Hence, the present results suggest that post-translational modifications, rather than gene expression, play a main role in the SOD antioxidant response of $X$. laevis lungs (Figure 3.10).

\subsection{Conclusions}

A recurring theme between the present chapter and Chapter $\mathbf{2}$ is that Sirt-related antioxidant signaling appears to be activated in the lungs of $X$. laevis as opposed to the brain, where it is comparatively inactive. This is evidenced by a strong activation in SIRT/PGC-1 $\alpha /$ FoxO signaling and downstream upregulations of catalase and HO-1 (Figures 3.1, 3.3, 3.5 and 3.6). These signaling pathways activated in the lungs likely contribute to an increase in catalase and HO-1 content (Figure 3.7), which allows the frog to better cope with dehydration-induced oxidative stress (Figure 3.10). By contrast, brain antioxidant signaling was unchanged with dehydration exposure, suggesting that an 
increase in blood circulation to this crucial organ may negate the need for the activation of antioxidant pathways. This is further supported by antioxidant capacity measurements, which show that dehydration depletes antioxidants in the lungs, but not the brain (Figure 3.8). Lastly, Sirt-mediated activation of antioxidants likely includes routes that do not implicate the FoxO transcription factors, such as the SIRT3-mediated deacetylation of SOD2 that is promoted by increased SIRT3 protein levels during dehydration (Figure $3.1)$. 


\subsection{References}

Awad, H., Nolette, N., Hinton, M., Dakshinamurti, S., 2014. AMPK and FoxO1 regulate catalase expression in hypoxic pulmonary arterial smooth muscle. Pediatr.

Pulmonol. 49, 885-897.

Baranano, D.E., Rao, M., Ferris, C.D., Snyder, S.H., 2002. Biliverdin reductase: A major physiologic cytoprotectant. Proc. Natl. Acad. Sci. 99, 16093-16098.

Chelikani, P., Fita, I., Loewen, P.C., 2004. Diversity of structures and properties among catalases. Cell. Mol. Life Sci. 61, 192-208.

Chen, Y., Zhang, J., Lin, Y., Lei, Q., Guan, K.L., Zhao, S., Xiong, Y., 2011. Tumour suppressor SIRT3 deacetylates and activates manganese superoxide dismutase to scavenge ROS. EMBO Rep. 12, 534-541.

Clark, J.E., Foresti, R., Green, C.J., Motterlini, R., 2000. Dynamics of haem oxygenase-1 expression and bilirubin production in cellular protection against oxidative stress. Biochem J 348 Pt 3, 615-619.

Daitoku, H., Hatta, M., Matsuzaki, H., Aratani, S., Ohshima, T., Miyagishi, M., Nakajima, T., Fukamizu, A., 2004. Silent information regulator 2 potentiates Foxo1mediated transcription through its deacetylase activity. Proc. Natl. Acad. Sci. 101, 10042-10047.

Emilio, M.G., Shelton, G., 1974. Gas exchange and its effect on blood gas concentrations in the amphibian, Xenopus laevis. J. Exp. Biol. 60, 567-579.

Hillman, S.S., 1978. The roles of oxygen delivery and electrolyte levels in the dehydrational death of Xenopus laevis. J. Comp. Physiol. B 128, 169-175.

Hillman, S.S., Sommerfeldt, R.W., 1981. Microsphere studies of amphibian systemic blood flow redistribution during dehydration, hypovolemia, and salt load. J. Exp. Zool. 218, 305-308.

Jacobs, K.M., Pennington, J.D., Bisht, K.S., Aykin-Burns, N., Kim, H.S., Mishra, M., Sun, L., Nguyen, P., Ahn, B.H., Leclerc, J., Deng, C.X., Spitz, D.R., Gius, D., 2008. SIRT3 interacts with the daf- 16 homolog FOXO3a in the mitochondria, as well as increases FOXO3a dependent gene expression. Int. J. Biol. Sci. 4, 291-299.

Kang, J., Jeong, M.G., Oh, S., Jang, E.J., Kim, H.K., Hwang, E.S., 2014. A FoxO1dependent, but NRF2-independent induction of heme oxygenase-1 during muscle atrophy. FEBS Lett. 588, 79-85.

Karnati, S., Lüers, G., Pfreimer, S., Baumgart-Vogt, E., 2013. Mammalian SOD2 is exclusively located in mitochondria and not present in peroxisomes. Histochem. Cell Biol. 140, 105-117.

Kawamata, H., Manfredi, G., 2010. Import, maturation, and function of SOD1 and its copper chaperone CCS in the mitochondrial intermembrane space. Antioxid. Redox 
Signal. 13, 1375-84.

Leiser, S.F., Kaeberlein, M., 2010. A Role for SIRT1 in the Hypoxic Response. Mol. Cell $38,779-780$.

Liu, G., Park, S.-H., Imbesi, M., Nathan, W.J., Zou, X., Zhu, Y., Jiang, H., Parisiadou, L., Gius, D., 2017. Loss of NAD-Dependent Protein Deacetylase Sirtuin-2 Alters Mitochondrial Protein Acetylation and Dysregulates Mitophagy. Antioxid. Redox Signal. 26, 849-863.

Loboda, A., Damulewicz, M., Pyza, E., Jozkowicz, A., Dulak, J., 2016. Role of Nrf2/HO1 system in development, oxidative stress response and diseases: an evolutionarily conserved mechanism. Cell. Mol. Life Sci.

Malik, A.I., Storey, K.B., 2011. Transcriptional regulation of antioxidant enzymes by FoxO1 under dehydration stress. Gene 485, 114-119.

Malik, A.I., Storey, K.B., 2009. Activation of antioxidant defense during dehydration stress in the African clawed frog. Gene 442, 99-107.

Matsuzaki, H., Daitoku, H., Hatta, M., Aoyama, H., Yoshimochi, K., Fukamizu, A., 2005. Acetylation of Foxol alters its DNA-binding ability and sensitivity to phosphorylation. Proc. Natl. Acad. Sci. 102, 11278-11283.

Motterlini, R., Foresti, R., Intaglietta, M., Winslow, R.M., 1996. NO-mediated activation of heme oxygenase: endogenous cytoprotection against oxidative stress to endothelium. Am. J. Physiol. 270, H107-14.

Olmos, Y., Sánchez-Gómez, F.J., Wild, B., García-Quintans, N., Cabezudo, S., Lamas, S., Monsalve, M., 2013. SirT1 Regulation of Antioxidant Genes Is Dependent on the Formation of a FoxO3a/PGC-1 $\alpha$ Complex. Antioxid. Redox Signal. 19, 1507-1521.

Qiu, X., Brown, K., Hirschey, M.D., Verdin, E., Chen, D., 2010. Calorie restriction reduces oxidative stress by SIRT3-mediated SOD2 activation. Cell Metab. 12, 662667.

Ryter, S.W., 2006. Heme Oxygenase-1/Carbon Monoxide: From Basic Science to Therapeutic Applications. Physiol. Rev. 86, 583-650.

Sundaresan, N.R., Gupta, M., Kim, G., Rajamohan, S.B., Isbatan, A., Gupta, M.P., 2009. Sirt3 blocks the cardiac hypertrophic response by augmenting Foxo3a-dependent antioxidant defense mechanisms in mice. J. Clin. Invest. 119, 2758-2771.

Tsang, C.K., Liu, Y., Thomas, J., Zhang, Y., Zheng, X.F.S., 2014. Superoxide dismutase 1 acts as a nuclear transcription factor to regulate oxidative stress resistance. Nat. Commun. 5, 3446.

Wang, X.X., Wang, X.L., Tong, M.M., Gan, L., Chen, H., Wu, S.S., Chen, J.X., Li, R.L., Wu, Y., Zhang, H.Y., Zhu, Y., Li, Y.X., He, J.H., Wang, M., Jiang, W., 2016. SIRT6 protects cardiomyocytes against ischemia/reperfusion injury by augmenting 
FoxO3 $\alpha$-dependent antioxidant defense mechanisms. Basic Res. Cardiol. 111, 1-19.

Wu, T.W., Wu, J., Li, R.K., Mickle, D., Carey, D., 1991. Albumin-bound bilirubins protect human ventricular myocytes against oxyradical damage. Biochem. Cell Biol. 69, 683-8. 


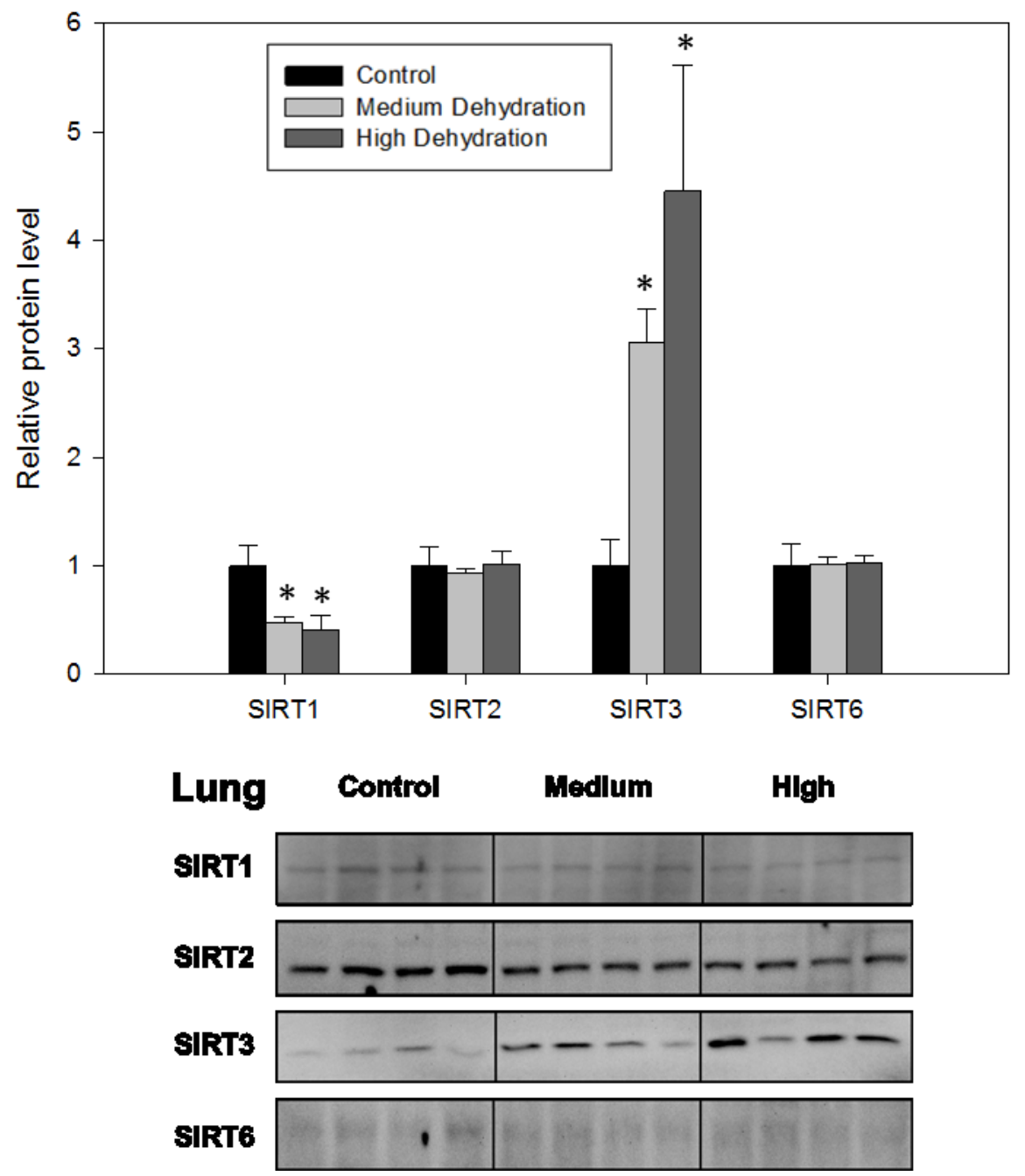

Figure 3.1. Immunoblotting was used to determine relative protein levels of 4 SIRT proteins known to play roles in antioxidant signaling in the lungs of $X$. laevis, comparing control, medium and high levels of whole-body dehydration. Chemiluminescent data derived from immunoblots was standardized against total protein loaded as represented by Coomassie staining of membranes. Histograms show means \pm SEM, $n=4$ independent biological replicates. Statistically significant differences compared to the control condition are denoted with an asterisk $(*)$ and were determined with a one-way ANOVA and post hoc Dunnett's test $(p>0.05)$. 

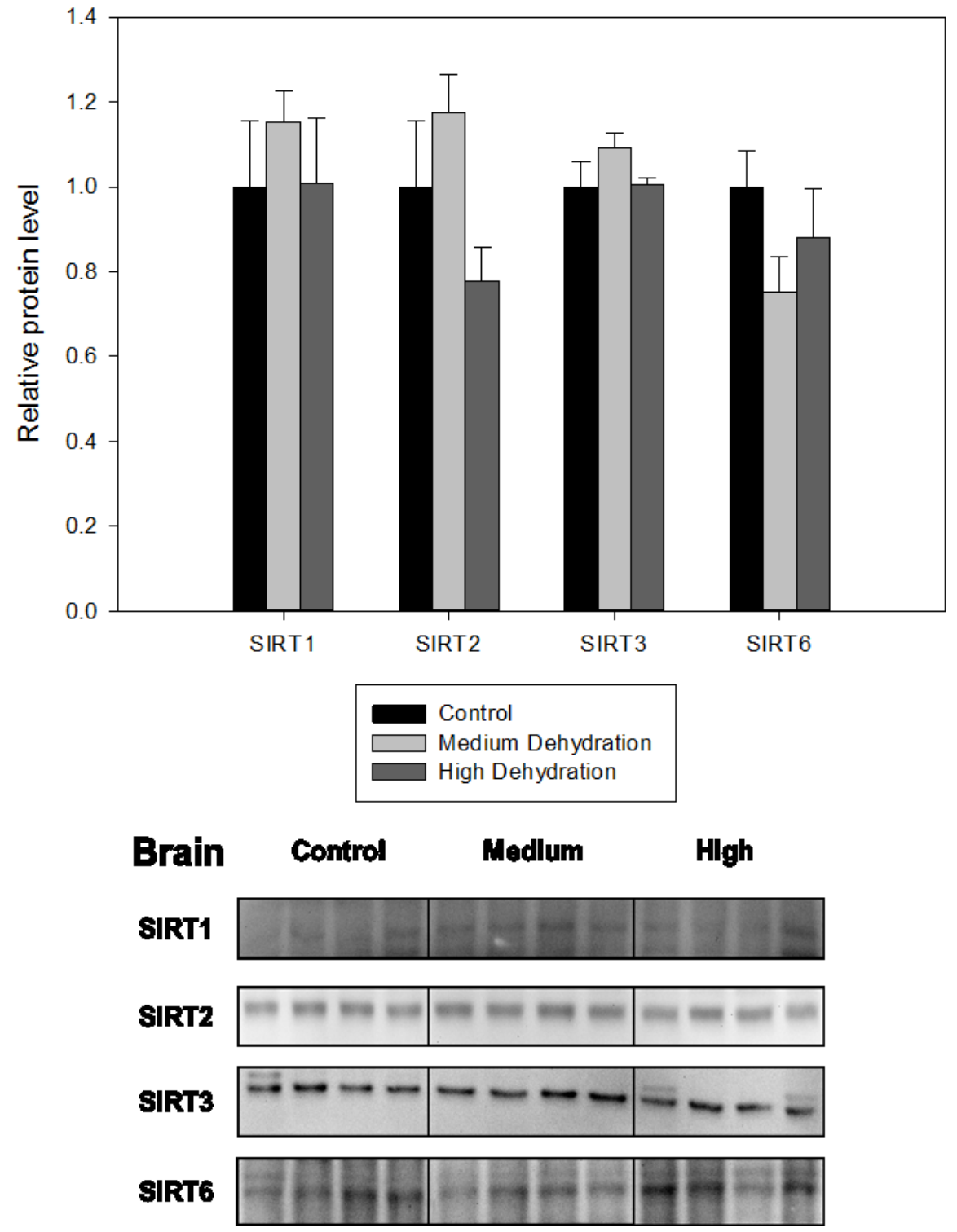

Figure 3.2. Immunoblotting was used to determine relative protein levels of 4 SIRT proteins known to play roles in antioxidant responses in the brains of $X$. laevis comparing control, medium and high levels of whole-body dehydration. Other information as in Figure 3.1. 

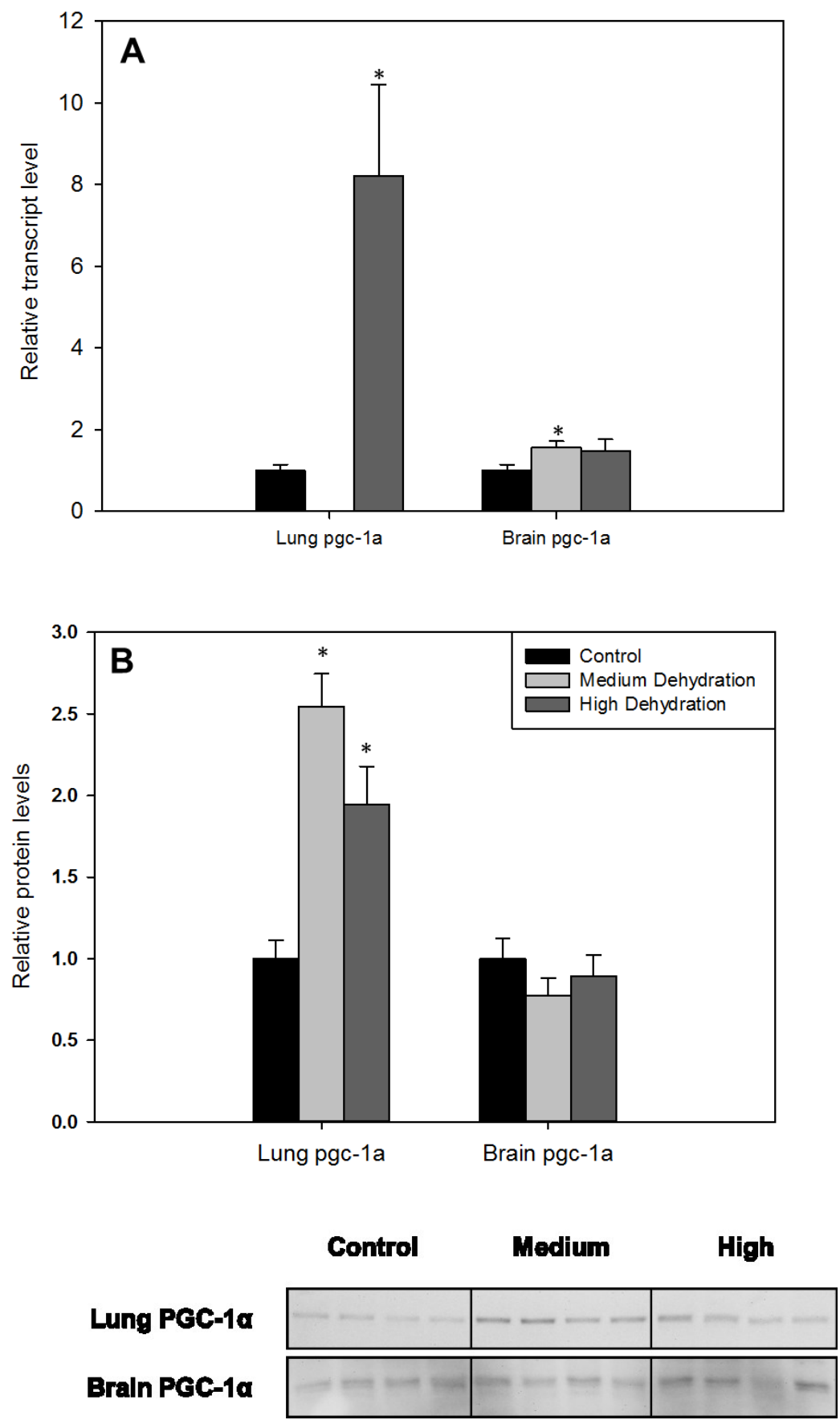

Figure 3.3. Relative mRNA (A) and protein (B) levels of PGC-1 $\alpha$ were assessed in the lungs and brains of $X$. laevis comparing control, medium and high levels of whole-body dehydration. Gene expression data was quantified with RT-qPCR and standardized against $r p l 27$ as a reference gene. Chemiluminescent data from immunoblots was standardized against total protein loaded as represented by Coomassie staining of membranes. Histograms show means \pm SEM, $n=4-6$ independent biological replicates. 


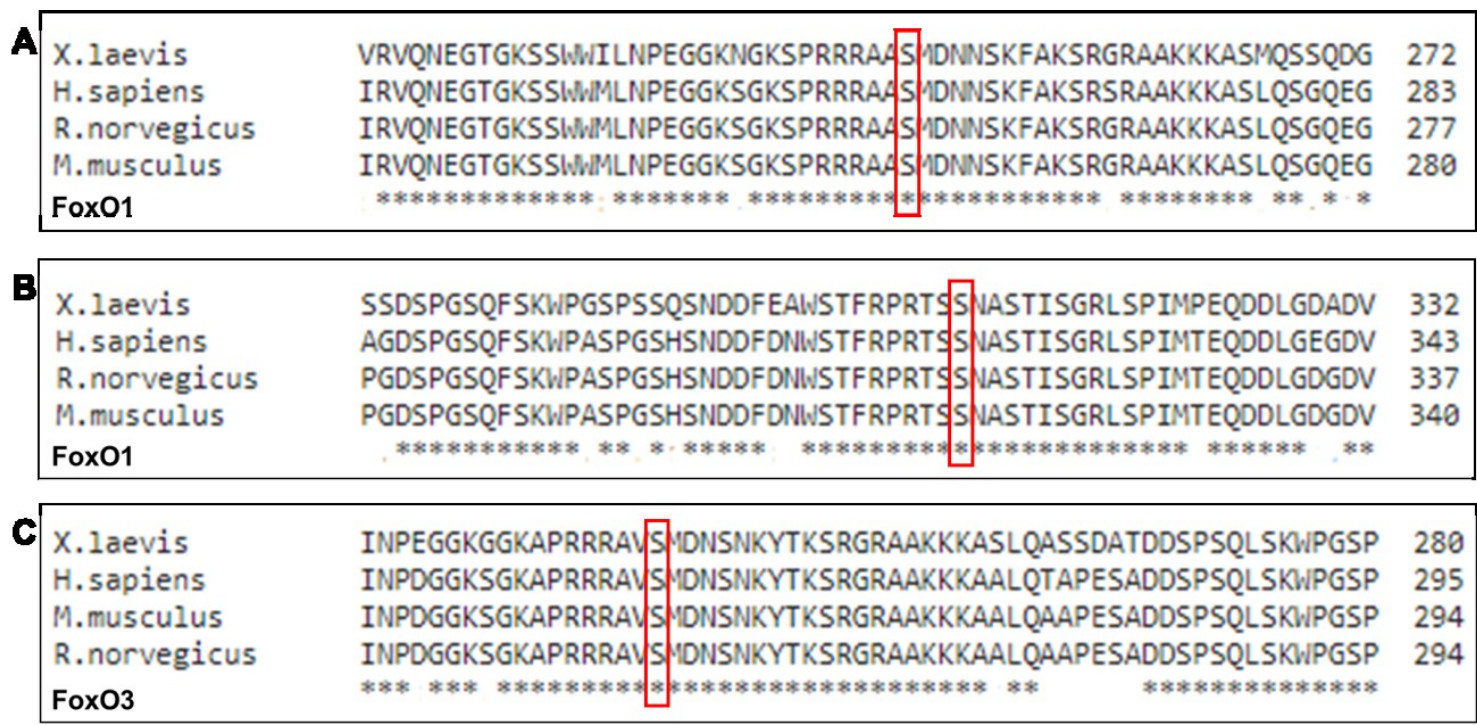

Figure 3.4. Partial primary structures of FoxO1 and FoxO3 in X. laevis, H. sapiens, $R$. norvegicus, and M. musculus. The phosphorylated Ser256 (A) and Ser319 (B) FoxO1 residues of humans are evolutionarily conserved as Ser245 and Ser308 in X. laevis. The conserved phosphorylated Ser253 (C) on human FoxO3 protein is also conserved as Ser238 in X. laevis. These conserved phosphorylation sites are shown in red boxes. 

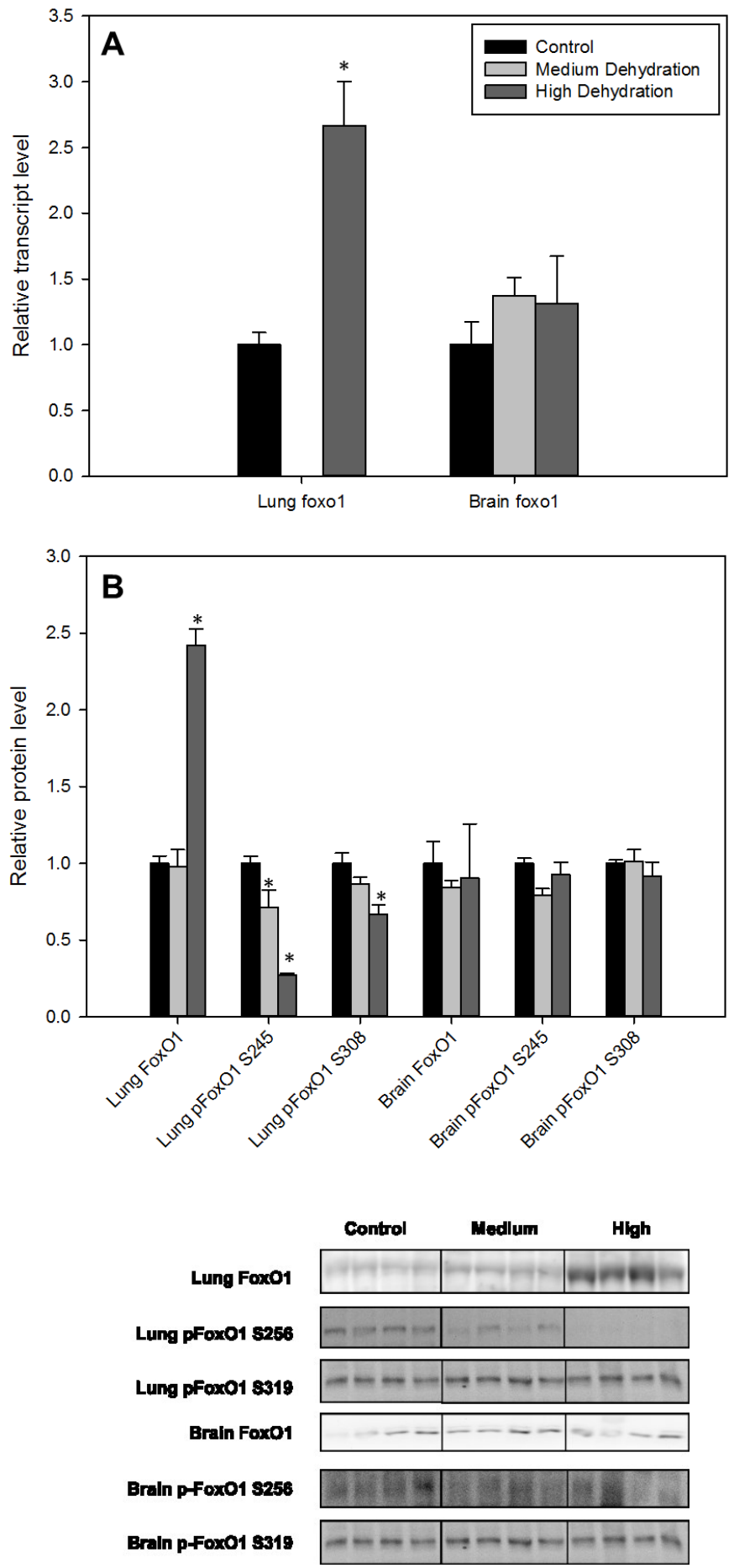

Figure 3.5. Relative mRNA (A) and protein (B) levels of FoxO1 were assessed in the lungs and brains of $X$. laevis comparing control, medium and high levels of whole-body dehydration. Other information as in Figure 3.3. 

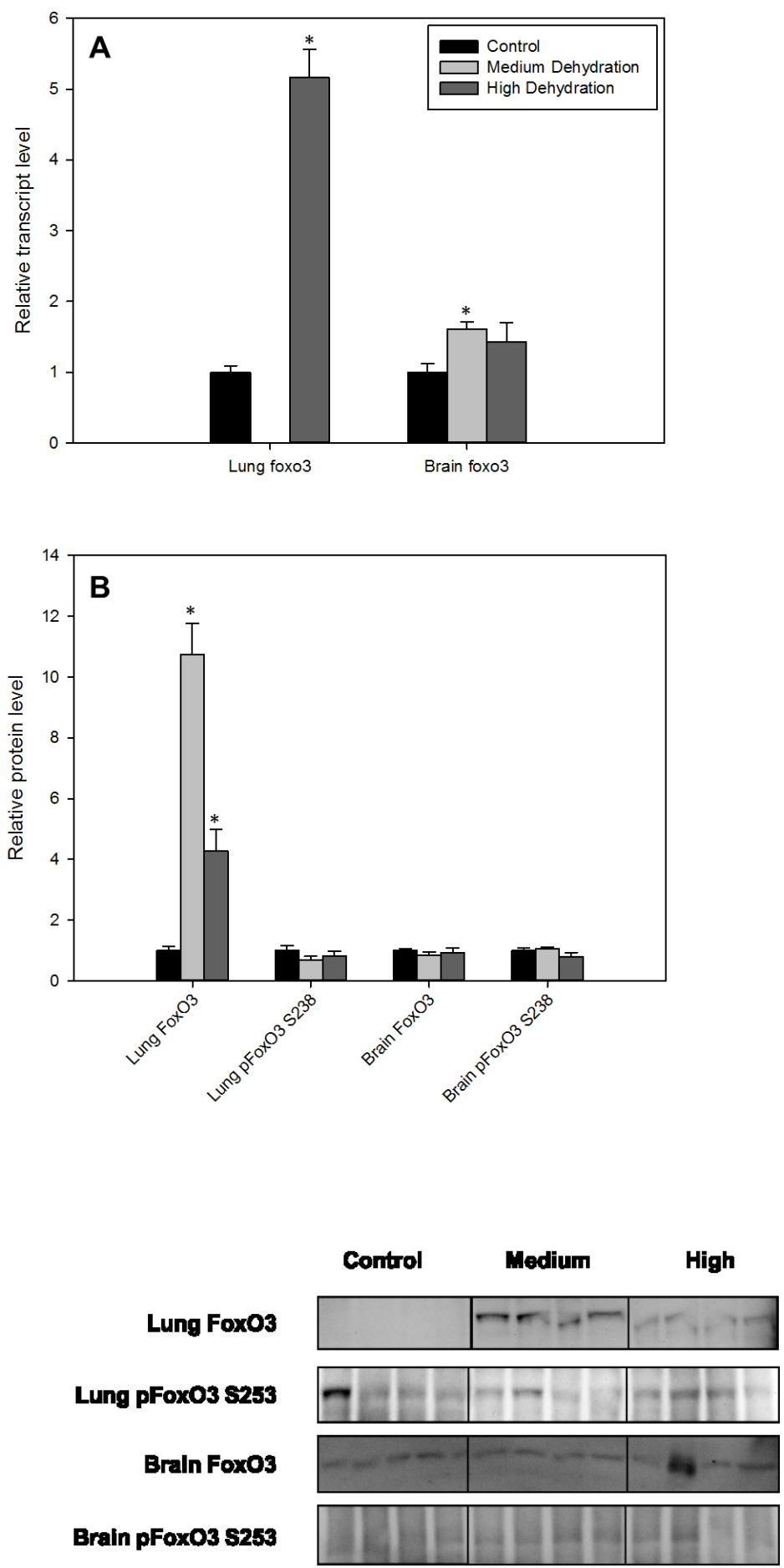

Figure 3.6. Relative mRNA (A) and protein (B) levels of FoxO3 were assessed in the lungs and brains of $X$. laevis comparing control, medium and high levels of whole-body dehydration. Other information as in Figure 3.3. 

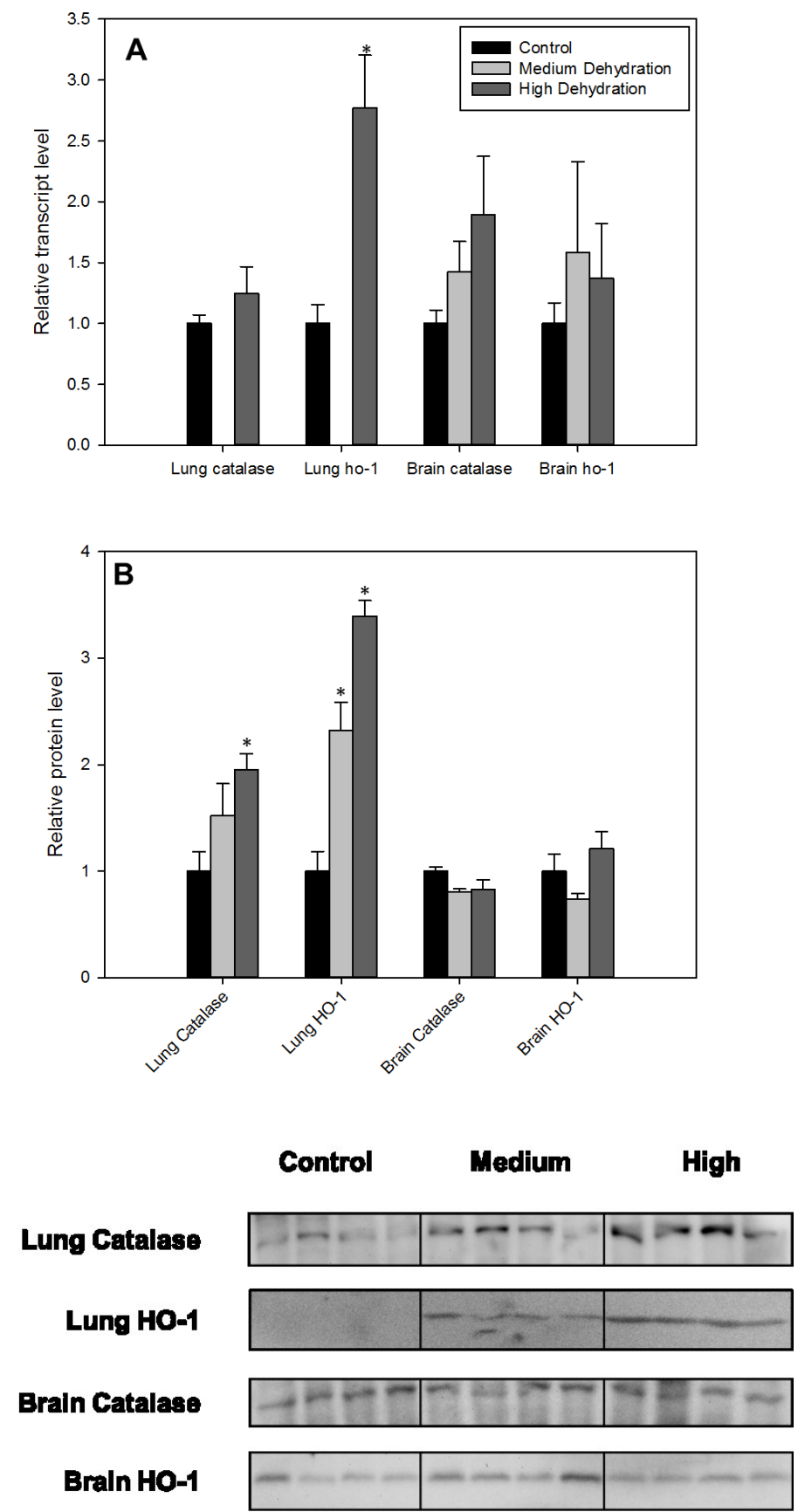

Figure 3.7. Relative mRNA (A) and protein (B) levels of Catalase and HO-1 were assessed in the lungs and brains of $X$. laevis comparing control, medium and high levels of whole-body dehydration. Other information as in Figure 3.3. 


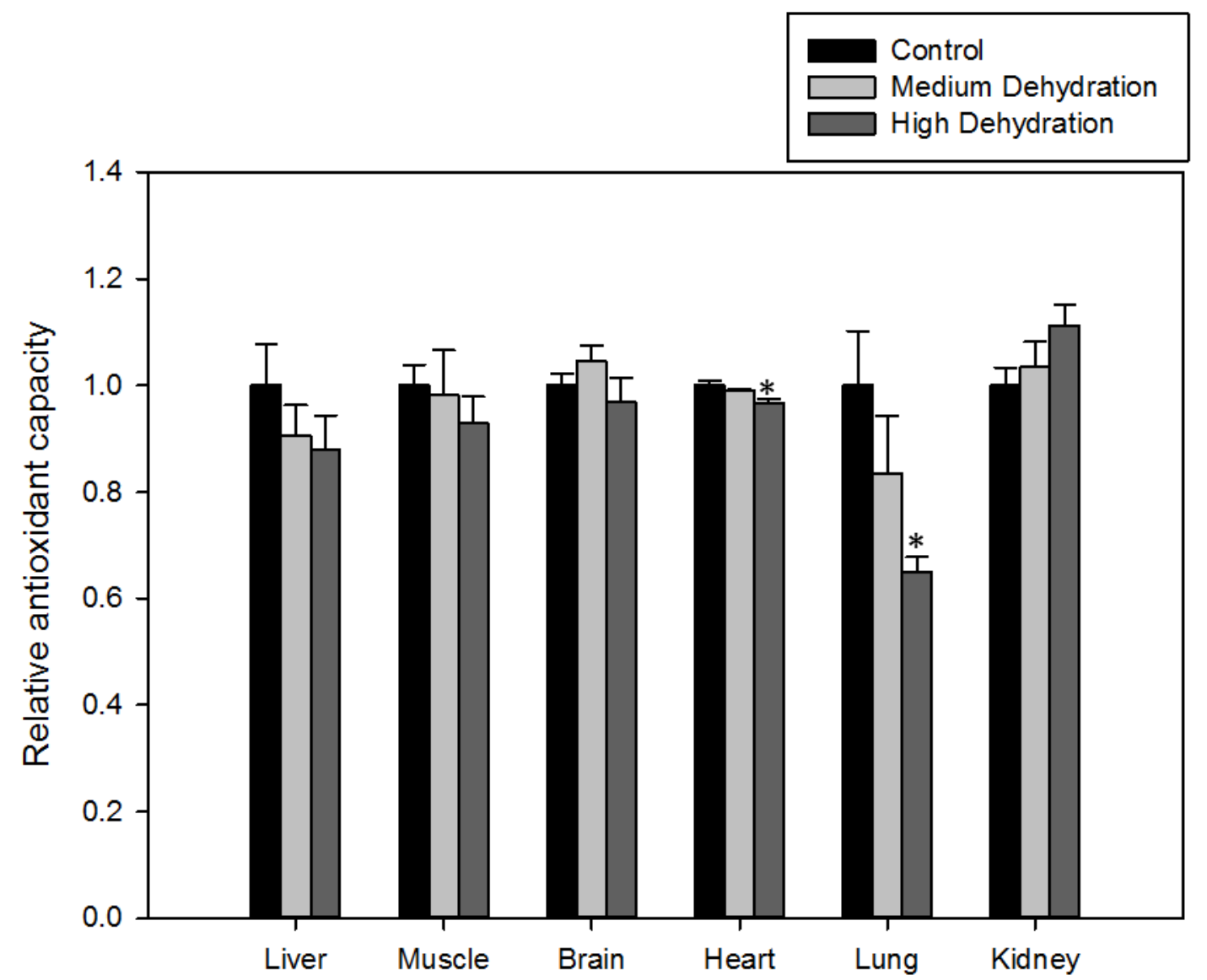

Figure 3.8. Relative antioxidant capacity of six $X$. laevis tissues comparing control, medium and high dehydration conditions. Assay results demonstrate the capacity of tissue samples to prevent oxidation of ABTS by metmyoglobin and hydrogen peroxide. Equal protein amounts were loaded for each biological replicate. Absorbance was measured at $405 \mathrm{~nm}$ and data was interpolated against a Trolox standard curve. Histograms show means \pm SEM, $n=4$ independent biological replicates. Statistically significant differences compared to the control condition are denoted with an asterisk $(*)$ and were determined with a one-way ANOVA and post hoc Dunnett's test $(p>0.05)$. 

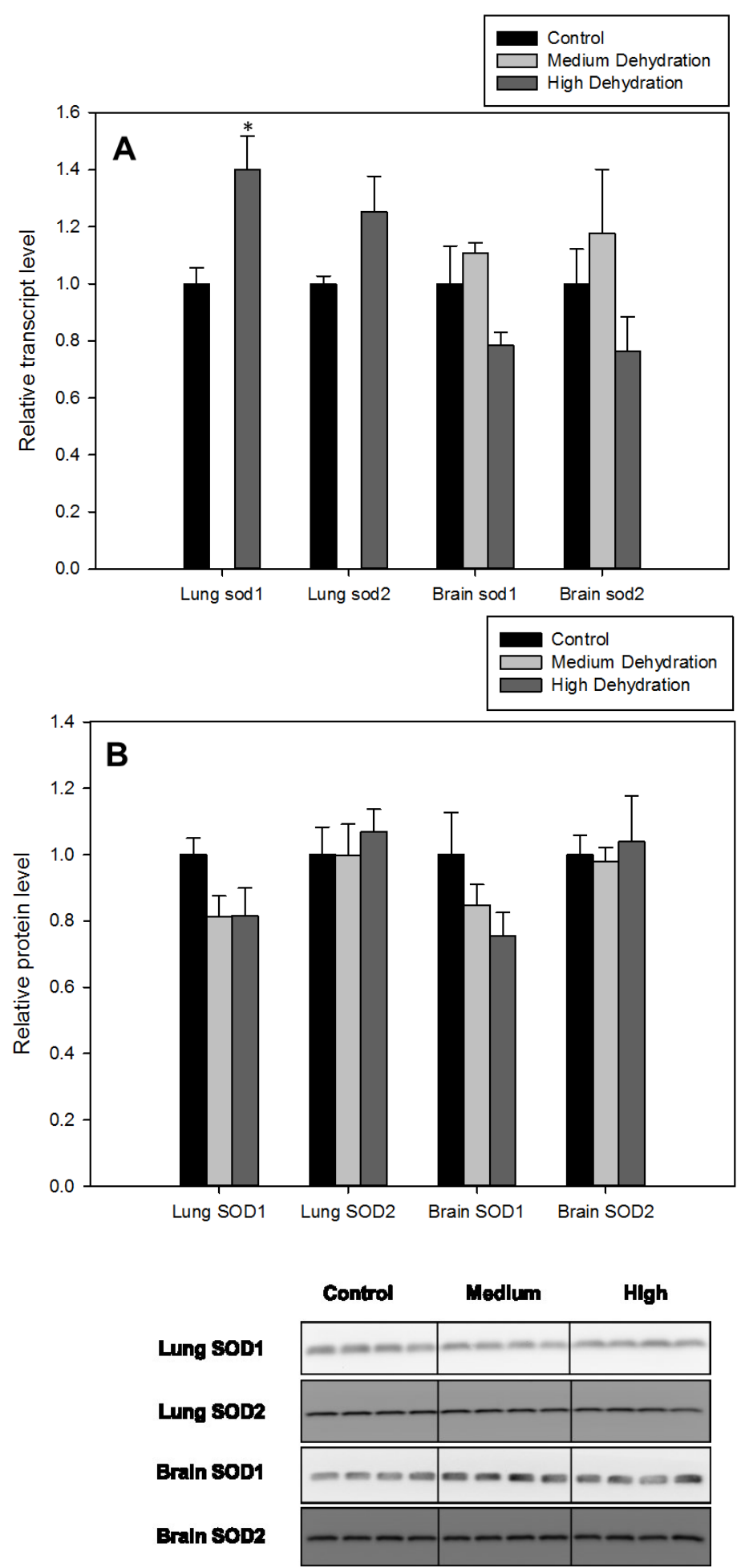

Figure 3.9. Relative mRNA (A) and protein (B) levels of SOD1 and SOD2 were assessed in lung and brain of $X$. laevis comparing control, medium and high levels of whole-body dehydration. Other information as in Figure 3.3. 


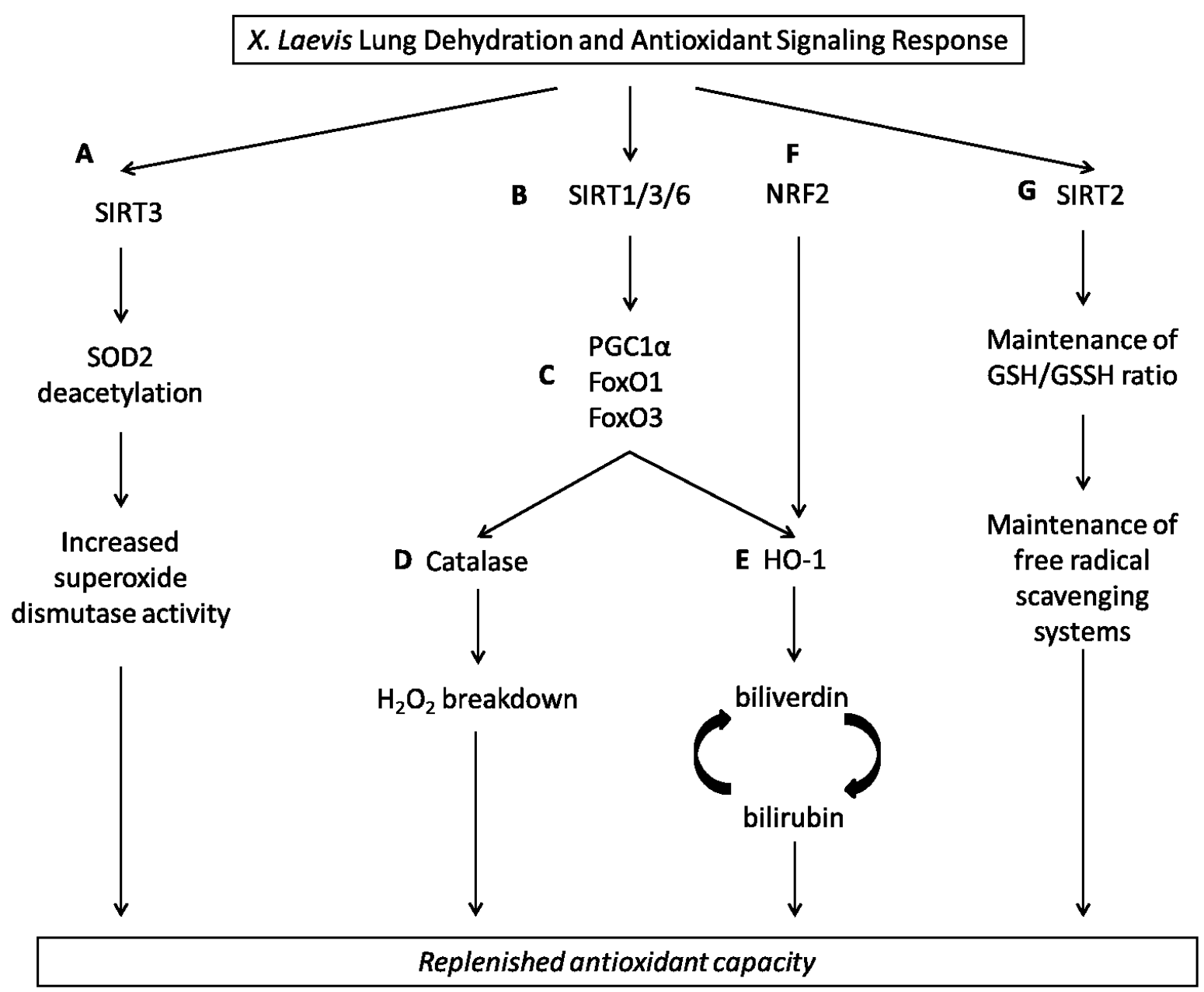

A. Increased global SIRT activity, total SIRT3 protein levels, decreased SOD2 acetylation status and constant total SOD2 mRNA/protein levels suggest a mechanism by which superoxide dismutase activity is increased during $X$. laevis whole-body dehydration.

B. Signaling by SIRT1/3/6 is generally activated as evidenced by general increases in global SIRT activity, increases in sirt 1 and sirt6, and increases in SIRT3 protein levels. Decreases in SIRT1 protein levels are known to be inducible by hypoxia in order to facilitate the upregulation HIF-1 $\alpha$ downstream genes.

C. Regulators of antioxidant enzyme expression are activated in response to dehydration, as evidenced by increased mRNA levels of pgc-1a, foxol, and foxo3. Correspondingly, protein levels of PGC-1 $\alpha$, FoxO1, and FoxO3 are also upregulated. Dehydration also results in a lower level of inhibitory phosphorylation on FoxO1 proteins, suggesting an increase in FoxO1 signaling.

D. Increases in SIRT1 and FoxO1/3 signaling contribute to the upregulation of catalase, as evidenced by increases in catalase mRNA and catalase protein levels. Catalase contributes to the $X$. laevis antioxidant system by facilitating the breakdown of $\mathrm{H}_{2} \mathrm{O}_{2}$. 
E. Increases in PGC-1 $\alpha$ during dehydration also correspondingly resulted in higher ho-1 mRNA and HO-1 protein levels. This enzyme contributes to the antioxidant system by creating biliverdin from heme degradation, which can be converted to bilirubin to act as a free radical scavenger.

F. The NRF2 transcription factor has been previously shown to be upregulated in $X$. laevis lungs during dehydration (Malik and Storey, 2009), and likely contributes to $\mathrm{HO}-1$ upregulation.

G. Increases in sirt2 mRNA during dehydration may play a role in sustaining $\mathrm{GSH} / \mathrm{GSSH}$ ratio for the scavenging of free radicals.

Figure 3.10. A schematic of the antioxidant signaling response in the lungs of $X$. laevis to dehydration showing the integration of data from the present chapter and that in Chapter 2 , and the established roles of the targets from in the literature. 


\title{
CHAPTER 4
}

\author{
PGC-1 $\alpha$ and Hif-1 $\alpha$-mediated Remodelling of \\ Metabolic Gene Expression during Dehydration
}




\subsection{Introduction}

\subsubsection{SIRTs and the regulation of metabolism}

The study of SIRT genes and SIRT deacetylase activity (Chapter 2) suggested that downstream metabolic pathways may be regulated in response to whole-body dehydration in X. laevis. In particular, an observed increase in sirt4 mRNA in the liver of X. laevis during dehydration may suggest a role for SIRTs in a broader mechanism by which the animal suppresses fatty acid oxidation. Knockdown of sirt4 in primary mouse hepatocyte cultures resulted in increased expression of genes responsible for facilitating fatty acid oxidation such as medium-chain acyl-CoA dehydrogenase ( $m c a d)$, carnitine palmitoyltransferase 1a (cptla), PPARgamma coactivator 1 alpha (pgcla), and peroxisome proliferator-activated receptor alpha (ppara) (Laurent et al., 2013; Nasrin et al., 2010). These effects were also observed in vivo, which showed that knockdown of SIRT4 increased fatty acid oxidation (Nasrin et al., 2010). Furthermore, the SIRT5 and SIRT3 proteins are known to deacetylate and increase the enzymatic activities of carbamoyl phosphate synthetase 1 (CPS1) and ornithine transcarbamylase (OTC), respectively, the first two enzymes of the urea cycle (Hallows et al., 2011; Nakagawa et al., 2009). Since an increase in global Sirt activity was found in the liver of dehydrated $X$. laevis, it can be hypothesized that a broad cellular response occurs during $X$. laevis dehydration to upregulate the ureagenesis pathway, which may include the expression of genes that encode for enzymes in the urea cycle. Indeed, urea levels have been shown previously to increase in dehydrated $X$. laevis (liver, muscle and plasma were measured), along with a 6-fold increase in enzymatic activity of CPS1, which was restored to normal levels with rehydration (Balinsky et al., 1967, 1961; Malik and Storey, 2011). The 
present chapter builds on the results from Chapter 2 and investigates the regulation of cellular pathways at the gene transcriptional level that control fatty acid and urea metabolism.

\subsubsection{The role of PGC-1 $\alpha$ in metabolism}

In Chapter 3, PGC-1 $\alpha$ was investigated for its role in antioxidant regulation. However, this transcriptional regulator has diverse roles, particularly in the control of metabolism. The roles of PGC-1 $\alpha$ in ureagenesis have been previously explored. In one study, overexpression of PGC-1 $\alpha$ was shown to facilitate the increase in sirt 3 and sirt 5 expression, which further increases the enzymatic activities of CPS1 and OTC (Li et al., 2016). In the present thesis, PGC-1 $\alpha$ is not only investigated for its role in antioxidants defense but also for its role in the metabolic regulation in dehydrating $X$. laevis. In addition to being a known regulator of SIRT3, SIRT5, and the urea cycle, PGC-1 $\alpha$ is also deacetylated and activated by SIRT1, thereby triggering multiple cellular responses such as antioxidants (Li et al., 2016; Olmos et al., 2013). In Chapter 2, sirt1 mRNA and global Sirt activity was found to be upregulated in the liver of dehydrated $X$. laevis. These results suggest that Sirt1-PGC-1 $\alpha$ signaling may also act as a key regulator of metabolism during whole-body dehydration.

In addition to the urea cycle, $\mathrm{PGC}-1 \alpha$ is also a major regulator of glucose metabolism and fatty acid oxidation. Upon deacetylation and activation by SIRT1, PGC$1 \alpha$ mediates the suppression of glycolytic genes (Rodgers et al., 2005). The activated PGC-1 $\alpha$ protein recruits another transcription factor, PPARa, to transcribe genes that are eventually translated into enzymes important in fatty acid oxidation. Fatty acid oxidation 
genes that are regulated by PGC-1 $\alpha /$ PPARa include acyl-CoA dehydrogenases and carnitine palmitoyltransferases (Nasrin et al., 2010; Purushotham et al., 2009).

\subsubsection{Conflicting roles for PGC-1 $\alpha$ in ischemia}

At this point, the paradoxical role of PGC-1 $\alpha$ in ischemia should be briefly overviewed: From one perspective, PGC-1 $\alpha$ induces the expression of genes involved in fatty acid oxidation - a process that would only logically proceed if oxygen was abundant. Under sufficiently oxygenated conditions in which the cells are capable of performing fatty acid oxidation, it is rational for PGC- $1 \alpha$ to express both antioxidant and fatty acid oxidation genes together in order to facilitate $\beta$-oxidation, but also to maintain or enhance antioxidant defenses as needed to effectively deal with the free radicals produced by the mitochondria (Figure 4.1).

From another standpoint, the PGC-1 $\alpha$ transcriptional coactivator was discussed in Chapter 2 for its potential role in the activation of antioxidants during dehydration, where oxygen transport to the cells is hampered. Indeed, it has been noted that the majority of studies on PGC- $1 \alpha$ have been performed under normoxic experimental conditions (Shoag and Arany, 2010). Furthermore, previous experiments have shown that pgcla expression is upregulated in hypoxia and ischemia-like conditions which are not favourable towards fatty acid oxidation (Shoag and Arany, 2010). Indeed, the roles of PGC-1 $\alpha$ in low oxygen environments are not yet fully understood and are growing areas of research. The PGC-1 $\alpha$ response has been shown to have a pivotal role in the control of vascular endothelial growth factor (vegf) gene expression, particularly in a Hif-1 $\alpha$ independent pathway, further suggesting that PGC-1 $\alpha$ has separate roles in low oxygen conditions (Arany et al., 2008) (Figure 4.1). In the context of dehydrating X. laevis, 
although the animal is expected to perform very little oxygen-dependent metabolism, this chapter will explore whether an activation of PGC-1 $\alpha$ expression occurs in multiple tissues, including the heart, liver, and skeletal muscles. This chapter will also reveal whether or not expected changes in gene expression patterns that support an increase in glycolytic processes and a decrease in fatty acid oxidation are observed.

\subsubsection{Hif-1 $\alpha$ promotes glycolysis and suppresses fatty acid oxidation in hypoxia}

Studies have suggested that PGC- $1 \alpha$ and Hif- $1 \alpha$ have independent gene expression roles. Despite sharing some common gene regulatory targets such as vegf, there are a number of reasons as to why the Hif- $1 \alpha$ transcription factor may be as equally important as PGC- $1 \alpha$ in the $X$. laevis dehydration response. The Hif- $1 \alpha$ transcription factor has also been shown to be regulated by deacetylases, specifically Sirt1. Sirt1 is required for Hif-1 $\alpha$ stabilization, and maintains stability by directly interacting with this transcription factor and protecting it from acetylation (Joo et al., 2015). Consistently, acetylation deactivates Hif- $1 \alpha$, resulting in the downregulation of its downstream targets including angiogenic targets such as vegf, and metabolic targets such as glucose transporters (Joo et al., 2015). Furthermore, Hif-1 $\alpha$ is known to induce the expression of glycolytic genes (Semenza, 2004), an effect that is hypothesized to be preferred over the oxygen-dependent fatty acid metabolism when $X$. laevis is under dehydrated conditions. Most importantly, Hif-1 $\alpha$ is known to inhibit the expression of the PPARa transcription factor, along with the expression of the mcad (medium chain) and lcad (long chain) genes that code for the acyl-CoA dehydrogenases that are required for $\beta$-oxidation (Huang et al., 2014) (Figure 4.2). This chapter will also explore whether or not the Hif-1 $\alpha$ response 
is activated during $X$. laevis dehydration, as a potential method for cells to suppress fatty acid oxidation genes in favour of expressing genes which favour glycolysis.

\subsubsection{Chapter objective}

The present chapter characterizes the Sirt1-activated PGC-1 $\alpha$ in the context of a metabolic regulator during $X$. laevis dehydration. To do this, a broad array of genes was quantified with RT-qPCR using RNA isolated from selected tissues of $X$. laevis under control and dehydrated states. Furthermore, key transcription factor targets were measured at the protein level with immunoblotting. Targets related to PGC-1 $\alpha$ were measured in order to assess the state of fatty acid oxidation, ureagenesis, angiogenesis, and glucose metabolism. Although PGC-1 $\alpha$ was hypothesized to be upregulated during dehydration, fatty acid oxidation was expected to be suppressed under low oxygen conditions. As a result, Hif-1 $\alpha$ was also assessed due to its known role in promoting glycolytic genes while suppressing those that promote fatty acid oxidation. Upstream regulators of PGC-1 $\alpha$ such as the myocyte enhancer factor-2 (MEF2) transcription factors were studied as a potential promoter of PGC-1 $\alpha$ expression in dehydration - a role they are known to have in diverse cell types and experimental conditions (Czubryt et al., 2003; Fernandez-Marcos and Auwerx, 2011; Handschin et al., 2003). Although related to PGC$1 \alpha$, PGC-1 $\beta$ was also assessed as it is known to be targeted in the Hif- $1 \alpha$-mediated suppression of lcad and mcad (Huang et al., 2014). The ppara, hepatocyte nuclear factor3-beta $(h n f 3 b)$, and hepatocyte nuclear factor-4-alpha ( $h n f 4 a)$ genes, known to promote the expression of acyl-CoA dehydrogenases and urea cycle enzymes, were measured to better understand how the cells of dehydrated X. laevis were regulating the expression of metabolic transcription factors during dehydration (Chen et al., 2017; Inoue et al., 2002; 
Laurent et al., 2013; Mandard et al., 2004; Wolfrum and Stoffel, 2006). Overall, this chapter provided evidence that PGC-1 $\alpha$ and Hif-1 $\alpha$ are important metabolic regulators in $X$. laevis dehydration which resulted in the remodelling of genes that is suggestive of increased glycolysis and decreased fatty acid oxidation. 


\subsection{Materials and Methods}

\subsubsection{Animals}

All animal experiments were conducted as described in Chapter 2.

\subsubsection{RNA extraction}

Isolation of RNA from X. laevis tissues was performed as described in Chapter 2.

\section{$\underline{4.2 .3 \text { cDNA synthesis }}$}

Synthesis of cDNA samples was performed as described in Chapter 2.

\section{$\underline{\text { 4.2.4 Primer design }}$}

Primers were designed for RT-qPCR as described in Chapter 2 and Appendix C for the following gene targets: pgcla, pgc1b, mef $2 a$, mef $2 c$, hnf $3 b, h n f 4 a, c p s 1$, otc, hifla, lcad, mcad, scad, cptla, cptlb, glut4, hk, pfk, pdk1, pdk3, pdk4, and vegf. Primer sequences can be found in Appendix $\mathbf{J}$.

\section{$\underline{4.2 .5 \mathrm{RT}-\mathrm{qPCR}}$}

RT-qPCR was performed as previously described in Chapter 2.

\subsubsection{Protein extraction}

Total protein extraction was performed as previously described in Chapter 2.

\subsubsection{Nuclear soluble protein isolation and extraction}

To prepare nuclear extracts, samples of frozen liver $(\sim 0.5 \mathrm{~g})$ or skeletal muscle $(\sim 1.0 \mathrm{~g})$ were homogenized using a Dounce homogenizer. Homogenization was in 1:2 w:v ratio of homogenization buffer (10 mM HEPES, pH 7.9, $10 \mathrm{mM} \mathrm{KCl,} 10 \mathrm{mM}$ EDTA, $1 \mathrm{mM}$ DTT, $20 \mathrm{mM}$ 2-glycerophosphate) with the addition of $10 \mu \mathrm{L}$ Protease Inhibitor Cocktail (Bio-Rad, Hercules, CA, USA) just prior to homogenization. Samples were centrifuged at $10,000 \times g$ for $10 \mathrm{~min}$ at $4^{\circ} \mathrm{C}$. The supernatants were retained as the cytoplasmic extracts. The pellets were then resuspended in $150 \mu \mathrm{L}$ of extraction buffer $(20$ 
mM Hepes, pH 7.9, 400 mM NaCl, 1 mM EDTA, 10\% v/v glycerol, 10 mM 2glycerophosphate, $1 \mathrm{mM}$ DTT) with $1.5 \mu \mathrm{L}$ of Protease Inhibitor Cocktail added just prior to the addition of the buffer to the pellet. Samples were incubated on ice for $1 \mathrm{~h}$. After the incubation, samples were centrifuged at $10,000 \times g$ for $10 \mathrm{~min}$ at $4^{\circ} \mathrm{C}$. The supernatants were retained as the soluble protein nuclear extracts. The protein concentrations of nuclear extracts were determined with the Bio-Rad protein assay and concentrations were adjusted to constant values with extraction buffer. To use samples for immunoblotting analysis of nuclear proteins, samples were treated with $2 \times$ SDS sample buffer as done for total protein extraction (4.2.6). The integrity of the nuclei was confirmed by immunoblotting of nuclear fractions with anti-histone H3 antibody; Cell Signaling). Cytoplasmic fractions were also comparatively assessed and were confirmed to have negligible histone H3 signal.

\subsubsection{Western immunoblotting}

Immunoblotting was performed as previously described in Chapter $\mathbf{2}$ with minor modifications. Antibodies were used to probe for PGC-1 $\alpha$, Hif-1a, MEF2A, and MEF2C. Specific experimental conditions that were used for each of the antibodies can be found in the Appendices $\mathbf{H} \& \mathbf{I}$. 


\subsection{Results}

\subsubsection{Liver}

A combination of RT-qPCR and immunoblotting was used to measure the relative levels of mRNA and protein of select targets that play metabolic roles in the liver. Characterization of transcription factors that are known to control metabolic targets were assessed in $X$. laevis liver from control, medium dehydration, and high dehydration conditions. Transcript levels of pgcla increased by $8.36 \pm 1.20$ fold under high dehydration conditions, as compared with controls, but there was no difference between control and medium dehydration (Figure 4.3A). However, total PGC-1 $\alpha$ protein levels in liver showed no change in response to dehydration (Figure 4.3B) (Note that results for skeletal muscle and heart are described in sections 4.3.2 and 4.3.3, respectively).

Secondly, the responses by MEF2 transcription factors were assessed in X. laevis liver, since these are known to activate PGC-1 $\alpha$ expression (Figures 4.4, 4.5 and 4.6). Liver mef $2 a$ transcript levels did not change with exposure to dehydration (Figure 4.4A). However, total MEF2A protein levels decreased significantly under high dehydration to $0.65 \pm 0.10$ of control levels, but no significant change in MEF2A nuclear protein levels were observed (Figure 4.4B and 4.4C). In contrast, liver mef2c transcript levels increased strongly in response to dehydration: compared to controls the levels rose by $21.61 \pm 7.41$ and $7.49 \pm 1.26$ fold in medium and high dehydration, respectively (Figure 4.5A). Despite this, total MEF2C protein levels remained unchanged during dehydration, whereas MEF2C levels were $1.45 \pm 0.10$ and $1.56 \pm 0.05$ fold higher in the nucleus under medium and high dehydration conditions, respectively (Figure 4.5B and 4.5C). 
Transcript levels of the $h n f 4 a$ and $h n f 3 b$ transcription factors were also assessed in $X$. laevis liver. Relative hnf4a mRNA levels decreased to $0.35 \pm 0.04$ of control levels during dehydration, whereas $h n f 3 b$ decreased to $0.27 \pm 0.01$ and $0.25 \pm 0.02$ of controls, in medium and high dehydration, respectively (Figure 4.7). Transcript levels for two enzymes of the urea cycle, cps1 and otc, were assessed. Dehydration-induced changes were only found during high dehydration, where there was a $2.15 \pm 0.14$ fold increase in cps 1 relative to the control (Figure 4.7). Dehydration did not result in any changes to otc transcript levels.

Transcript levels of another major transcription factor, hif-1a, were observed to increase by $1.60 \pm 0.06$ in the $X$. laevis liver under high dehydration (Figure $4.8 \mathrm{~A}$ ). Immunoblots also revealed that liver Hif- $1 \alpha$ protein levels also increased by $3.34 \pm 0.23$ fold in high dehydration (Figure 4.8B).

The ppara transcription factor was also assessed in $X$. laevis liver. Transcript levels of ppara decreased during both medium and high dehydration, to $0.67 \pm 0.02$ and $0.51 \pm 0.07$ of control levels, respectively (Figure 4.9A). Levels of $p g c 1 b$ were also dehydration responsive in the liver, increasing $2.20 \pm 0.35$ and $1.90 \pm 0.46$ fold in medium and high dehydration, respectively (Figure 4.9B).

In order to shed light on the metabolic responses to whole-body dehydration at the gene expression level, transcripts of select genes involved in fatty acid and glucose metabolism were measured in the liver of dehydrated $X$. laevis. Relative transcript levels of acyl-CoA dehydrogenases and carnitine palmitoyltransferases were quantified in order to investigate the differential expression of proteins involved in fatty acid oxidation. In 
response to high dehydration, lcad was found to decrease to $0.43 \pm 0.07$ and $0.62 \pm 0.04$ in medium and high dehydration conditions, respectively (Figure 4.10A). Liver mcad was only responsive in high dehydration, decreasing to $0.62 \pm 0.04$ of control levels but scad did not change with dehydration (Figure 4.10A). The transcript levels of cpt $1 a$ and cpt $1 b$ genes were also measured, but no changes were observed in liver with exposure to dehydration (Figure 4.11A). Target genes involved in glycolysis and glucose consumption were also assessed, including glucose transporter type 4 (glut4), hexokinase (hk), phosphofructokinase ( $p f k)$, pyruvate dehydrogenase kinase 1 ( $p d k 1), p d k 3$ and $p d k 4$. A $1.63 \pm 0.14$ fold increase in liver $h k$ mRNA was observed in high dehydration, but levels in medium dehydration were not different from the control (Figure 4.12). Similarly, transcript levels of $p d k 3$ and $p d k 4$ also increased in high dehydration by1.52 \pm 0.16 and $6.93 \pm 0.41$ fold relative to the control, with no changes seen in medium dehydration (Figure 4.12). Transcript levels of glut4, pfk and $p d k l$ in liver did not change in the experimental conditions assessed.

\section{$\underline{\text { 4.3.2 Skeletal muscle }}$}

As done for liver, transcription factor targets implicated in the regulation of metabolism were also assessed in the skeletal muscle of $X$. laevis under control and dehydrated states. Transcript levels of muscle $p g c l a$ were found to decrease to $0.23 \pm 0.03$ and $0.46 \pm 0.10$ of control levels in medium and high dehydration, respectively (Figure 4.3A). Consistently, PGC-1 $\alpha$ protein levels in skeletal muscle decreased to $0.64 \pm 0.05$ and $0.59 \pm 0.05$ of control levels, in medium and high dehydration (Figure 4.3B).

Dehydration resulted in a decrease in skeletal muscle mef $2 a$ and $m e f 2 c$ transcript levels (Figure 4.6). Medium dehydration caused mef2a mRNA levels to decrease to 
$0.49 \pm 0.05$, but no changes were seen between the controls and high dehydration (Figure 4.4A). As for $m e f 2 c$, medium and high dehydration decreased the transcript levels of this gene to $0.32 \pm 0.01$ and $0.09 \pm 0.09$ of controls, respectively (Figure $4.5 \mathrm{~A}$ ). Total and nuclear MEF2A protein levels were not sensitive to dehydration (Figure 4.4B and 4.4C). Although dehydration did not change total MEF2C levels in muscle, high dehydration reduced nuclear MEF2C protein levels to $0.74 \pm 0.06$ of controls (Figure 4.5B and 4.5C).

Transcript levels of the hypoxia-inducible transcription factor gene, hifla, was unresponsive to medium dehydration in skeletal muscle, but increased by $1.63 \pm 0.23$ fold under high dehydration (Figure 4.8A). Correspondingly, muscle Hif-1 $\alpha$ protein levels also increased by $1.53 \pm 0.12$ fold in high dehydration (Figure 4.8B). Regarding the regulation of fatty acid oxidation, transcript levels of ppara were downregulated to $0.48 \pm 0.07$ and $0.61 \pm 0.14$ of controls in medium and high dehydration, respectively (Figure 4.9A). In contrast, skeletal muscle $p g c l b$ transcripts increased by $2.41 \pm 0.24$ fold during high dehydration, but did not change during medium dehydration (Figure 4.9B).

Relative expression of genes encoding for acyl-CoA dehydrogenases were generally downregulated in skeletal muscle in response to dehydration. Transcript levels of lcad decreased in both medium and high dehydration to $0.31 \pm 0.05$ and $0.56 \pm 0.05$ of control levels, respectively (Figure 4.10B). Likewise, mcad mRNA also decreased in both medium and high dehydration, to $0.57 \pm 0.05$ and $0.59 \pm 0.02$ of controls (Figure 4.10B). Transcript levels of $s c a d$ decreased to $0.59 \pm 0.02$ of controls under medium dehydration, but did not change with respect to controls in high dehydration (Figure 4.10B). In contrast to the regulation of acyl-CoA dehydrogenase genes in skeletal muscle, cpt1 transcripts were upregulated in response to dehydration. Medium and high 
dehydration resulted in $2.08 \pm 0.24$ and $1.72 \pm 0.26$ fold increases in cptla mRNA, respectively (Figure 4.11B). Transcript levels of $c p t 1 b$ also increased under high dehydration to $1.87 \pm 0.03$ of control levels (Figure 4.11B). Genes coding for glycolytic enzymes in skeletal muscle decreased, with $h k$ downregulated to $0.59 \pm 0.03$ and $0.72 \pm 0.08$ of control levels, during medium and high dehydration, respectively (Figure 4.13). Comparable reductions in $p f k$ transcripts were seen with levels reduced to $0.40 \pm 0.03$ and $0.66 \pm 0.07$ in medium and high dehydration, respectively (Figure 4.13). Transcripts of muscle $p d k 3$ increased $5.00 \pm 1.46$ fold compared to controls with high dehydration and even larger increases were recorded for $p d k 4$ with transcript levels increasing by $9.49 \pm 0.03$ and $12.91 \pm 0.75$ fold above control levels during medium and high dehydration (Figure 4.13). Although glut4 and pdkl mRNA levels were assessed, they were not responsive to dehydration.

\subsubsection{Heart}

Transcript levels of pgcla in the heart of $X$. laevis decreased to $0.49 \pm 0.05$ of control levels during medium dehydration, and to $0.55 \pm 0.10$ during high dehydration (Figure 4.3A). Similarly, protein levels of PGC-1 $\alpha$ also decreased in the heart to $0.69 \pm 0.04$ and $0.60 \pm 0.04$ of control levels, during medium and high dehydration, respectively (Figure 4.3B).

Dehydration resulted in reduced transcript levels of mef2a to $0.64 \pm 0.03$ and $0.58 \pm 0.05$ of control levels during medium and high dehydration, respectively (Figure 4.4A). Similarly, $m e f 2 c$ decreased to $0.27 \pm 0.13$ of controls during high dehydration, but was not affected by medium dehydration (Figure 4.5A). Protein levels of heart MEF2A were unchanged with exposure to dehydration (Figure 4.4B) but MEF2C protein levels 
decreased to $0.71 \pm 0.04$ in high dehydration (Figure 4.5B). Due to a very limited amount of heart tissue, the nuclear content of MEF2A and MEF2C could not be evaluated.

High dehydration resulted in a $4.00 \pm 0.44$ increase in heart hifla transcript levels, but medium dehydration had no effect (Figure 4.8A). Unlike liver and muscle, dehydration did not change ppara mRNA levels in heart (Figure 4.9A). In contrast, pgclb transcript levels increased $1.51 \pm 0.07$ fold in medium dehydration, but were unchanged in high dehydration as compared to the control (Figure 4.9B).

Transcript levels of mcad increased by $1.59 \pm 0.18$ fold over controls in medium dehydration, but decreased again to $0.61 \pm 0.04$ of control levels in high dehydration (Figure 4.10C). Similarly, scad increased by $1.52 \pm 0.09$ fold under medium dehydration, but decreased to $0.52 \pm 0.02$ of controls in high dehydration. Transcript levels of heart cpt $1 b$ increased to $1.80 \pm 0.31$ during medium dehydration, but high dehydration did not show a difference from controls (Figure 4.11C). No changes to lcad (Figure 4.10C) or cptla mRNA levels were induced by dehydration in the heart (Figure 4.11C).

With respect to glucose metabolism, heart glut4 transcript levels decreased under medium and high dehydration conditions, to $0.27 \pm 0.11$ and $0.08 \pm 0.02$ of controls, respectively (Figure 4.14). Transcripts of $h k$ did not change in medium dehydration, but decreased to $0.57 \pm 0.09$ of controls during high dehydration, and similarly, $p f k$ transcript levels were also unchanged in medium dehydration, but dropped to $0.53 \pm 0.03$ of controls in high dehydration (Figure 4.14). Transcripts of heart $p d k 4$ increased $5.56 \pm 0.42$ fold in medium dehydration, and 6.67 \pm 1.67 fold in high dehydration but heart $p d k 1$ and $p d k 3$ transcripts did not change (Figure 4.14). 


\subsubsection{Hif- $1 \alpha$ in other tissues and VEGF mRNA}

In addition to liver, muscle, and heart, hifla was also measured in kidney, lung, and brain. Although no changes in mRNA were found in the kidney and brain, lung hifla increased $1.58 \pm 0.11$ fold during high dehydration (Figure 4.8A). Likewise, lung Hif-1 $\alpha$ protein levels also increased in high dehydration by $1.51 \pm 0.12$ fold of controls (Figure 4.8B).

The majority of targets assessed are known for their roles in metabolism. The vegf gene, which is also controlled by both PGC-1 $\alpha$ and Hif-1 $\alpha$, was assessed for its role in angiogenesis, a potential response to dehydration. Transcript levels of vegf were assessed in 6 tissues (liver, muscle, heart, kidney, lung, and brain) of $X$. laevis under control and dehydrated states. In the liver, vegf increased by $2.81 \pm 0.20$ fold in high dehydration, but levels were not significantly higher than controls under medium dehydration (Figure 4.15). Interestingly, vegf decreased with high dehydration in both heart $(0.46 \pm 0.10)$ and lungs $(0.58 \pm 0.07)$, compared to their respective controls (Figure 4.15). Dehydration did not result in any changes to vegf transcript levels in the muscle, brain, or kidneys. 


\subsection{Discussion}

The present chapter characterized the Sirtuin and MEF2-controlled transcriptional regulators, PGC-1 $\alpha$ and Hif-1 $\alpha$, on both mRNA and protein levels. Activation of these transcription factors is known to have implications on the control of diverse genes that have crucial metabolic roles. In addition to the assessment of these 4 targets, other factors that were assessed include $h n f 4 a, h n f 3 b, p g c l \beta$, and ppara as they are known to control metabolic processes linked to PGC-1 $\alpha$ and Hif-1 $\alpha$. These key transcription factors are known to control genes involved in glucose (glut4, $h k, p f k, p d k 1, p d k 3$ and $p d k 4)$, fatty acid (cptla, cpt1b, lcad, mcad, and scad), and urea metabolism (cps1 and otc). Measurements were done on multiple $X$. laevis tissues under control and dehydration conditions, but focused primarily on the liver, skeletal muscle, and heart. The results showed that dehydration leads to the remodelling of transcription factors that is suggestive of increased ureagenesis and decreased fatty acid oxidation in favour of glucose metabolism, as evidenced by changes in gene expression patterns.

\subsubsection{PGC-1 $\alpha$ in the liver, muscle and heart}

This chapter assessed PGC-1 $\alpha$ at both the mRNA transcript and protein levels in the liver, muscle and heart of $X$. laevis undergoing whole-body dehydration. These assessments were performed in order to further our understanding of this frog's metabolism during dehydration. The measurements showed that pgcla mRNA significantly increased in the liver, but decreased in the muscle and heart (Figure 4.3A). In addition, protein amounts of PGC- $1 \alpha$ remained constant in the liver, but decreased in the muscle and heart when encountering whole-body dehydration (Figure 4.3B). This suggests that PGC-1 $\alpha$ may have a liver-specific role during dehydration, which warrants 
the observed increase in $p g c l \alpha$ transcription that may have an effect in sustaining the relative amount of PGC-1 $\alpha$ protein throughout dehydration exposure. By contrast, a reduction in both relative mRNA and protein levels of PGC-1 $\alpha$ in the muscle and heart suggest that a suppression of this transcriptional regulator is part of a broader $X$. laevis dehydration response.

Results from Chapter 2 showed that global Sirtuin deacetylase activity increases in a number of tissues during $X$. laevis whole-body dehydration, including the liver, skeletal muscle, and heart. In order to further understand how PGC-1 $\alpha$, a target of sirtuin action, is itself regulated during dehydration, other upstream regulators, namely the MEF2 transcription factors, were characterized to determine their responses to dehydration. The MEF2 transcription factors are known to bind to the pgcla promoter region, and in doing so, increase the transcription of pgcla mRNA (Czubryt et al., 2003; Fernandez-Marcos and Auwerx, 2011; Handschin et al., 2003). In this chapter, the MEF2A and MEF2C transcription factors were profiled at both mRNA and protein levels in liver, muscle and heart of dehydrating $X$. laevis. In the liver, mef $2 c$ transcript levels were considerably upregulated during dehydration, as opposed to the muscle and heart, where mef $2 c$ decreased (Figure 4.5A). Although total MEF2C protein levels did not respond to dehydration in the liver (Figure 4.5B), the nuclear content of MEF2C protein increased (Figure 4.5C). The observed increases in mef $2 c$ mRNA and MEF2C nuclear protein levels suggest an active role for MEF2C in the liver. Indeed, elevated nuclear protein levels are suggestive of increased promoter binding and gene expression of MEF2C downstream targets - one known target being the pgcla gene (Czubryt et al., 2003; Handschin et al., 2003). This contrasts with the skeletal muscles, in which MEF2C 
mRNA and nuclear protein levels were observed to decrease with exposure to dehydration (Figure 4.5). However, the MEF2C data for both liver and muscle were consistent with the results for PGC-1 $\alpha$, and suggest that MEF2C may be increasing pgcla expression in the liver, but not the muscle during dehydration. With respect to MEF2C levels, the heart responded similarly to skeletal muscle and was consistent with a decrease in heart PGC-1 $\alpha$ mRNA and protein. Although nuclear protein levels were not assessed due to limited heart tissue, $m e f 2 c$ mRNA and total MEF2C protein were observed to decrease in response to high dehydration (Figure 4.5).

In addition to MEF2C, MEF2A was also assessed in the liver, muscle and heart. Unlike MEF2C, liver MEF2A transcript and nuclear protein levels were unchanged, whereas total protein decreased under high dehydration (Figure 4.4). Reduction in mef2a mRNA and protein were observed for muscle and heart, but particularly for the liver, it can be postulated that MEF2C plays a larger role than MEF2A in the regulation of PGC$1 \alpha$ that occurs during whole-body dehydration in $X$. laevis. From these results alone however, a role for MEF2A in the regulation of pgcla during dehydration cannot be ruled out as differences in binding of transcription factors to gene promoters were not directly measured. Overall, the results support the notion that dehydration-mediated changes to MEF2C may contribute to PGC-1 $\alpha$ upregulation in the liver and suppression in the muscle and heart.

\subsubsection{Regulation of ureagenesis by PGC-1 $\alpha$}

As previously discussed, overexpression of PGC- $1 \alpha$ is known to induce ureagenesis by upregulating sirt 3 and sirt5, which in turn deacetylate and activate the CPS1 and OTC enzymes of the urea cycle (Hallows et al., 2011; Inoue et al., 2002; 
Nakagawa et al., 2009). An increase in pgcla mRNA expression in the liver during dehydration suggests that pgcla likely contributes to the increased ureagenesis that is known to occur in X. laevis (Balinsky et al., 1967, 1961; Malik and Storey, 2011). To further understand the molecular signaling pathways that may be contributing to urea cycle activation, mRNA transcript levels of $h n f 3 b$ and $h n f 4 a$ as well as cps 1 and otc were quantified in liver. The hepatocyte nuclear factors HNF3 $\beta$ and HNF4 $\alpha$ are transcription factors that induce the mRNA expression of cps 1 and otc by binding to their respective promoter regions (Chen et al., 2017; Inoue et al., 2002). Although otc was unchanged, mRNA levels of cps 1 increased in the liver of dehydrated $X$. laevis, demonstrating that increases in urea cycle genes contribute to the broader dehydration response. However, $h n f 3 b$ and $h n f 4 a$ were both downregulated in response to dehydration in liver (Figure 4.7), suggesting that these transcription factors may not be the ones involved in ureagenesis by promoting the expression of $c p s 1$ and otc. Overall, the results from this chapter provided evidence that ureagenesis in dehydrating $X$. laevis involves increased expression of genes that translate into CPS1, and may also involve to the deacetylase activity of liver Sirts, which were found to increase in Chapter 2, and which have the potential to modify posttranslational modifications and enzymatic activity of CPS1.

\section{$\underline{4.4 .3}$ Regulation of fatty acid metabolism by PGC- $1 \alpha / \beta$ and Hif- $1 \alpha$}

Although HNF3 $\beta$ and HNF4 $\alpha$ are known to contribute to ureagenesis, they also broadly regulate diverse genes in the liver, including those that facilitate lipid metabolism in hepatocytes by inducing the expression of genes involved in fatty acid oxidation (Martinez-Jimenez et al., 2010; Wolfrum and Stoffel, 2006). The suppression of these genes during dehydration may facilitate a decrease in $\beta$-oxidation, which is a logical 
response when animals are faced with impaired oxygen delivery as dehydration becomes more severe. As previously discussed, PGC-1 $\alpha$ activation in the liver during dehydration, could have a central role in upregulating mitochondrial genes including those that facilitate fatty acid oxidation. However, it would not appear to be logical for X. laevis to activate $\beta$-oxidation through PGC-1 $\alpha$ during dehydration.

In order to better understand the molecular mechanisms of fatty acid oxidation during $X$. laevis dehydration, Hif-1 $\alpha$ protein and transcripts were investigated along with ppara and $p g c 1 b$. Under conditions of low oxygen, Hif- $1 \alpha$ is able to suppress the expression of ppara and pgclb (Huang et al., 2014; Shoag and Arany, 2010). The ppara gene encodes a transcription factor that stimulates the transcription of downstream genes important in fatty acid oxidation, including lcad, mcad, scad, cptla, and cpt1b (Lovekamp-Swan et al., 2003; Nasrin et al., 2010; Purushotham et al., 2009). Furthermore, Hif- $1 \alpha$ also reduces fatty acid oxidation by suppressing lcad and mcad expression levels via downregulation of pgclb (Huang et al., 2014). Dehydrated X. laevis showed increased hifla mRNA levels in the liver, muscle, heart, and lungs (Figure 4.8A). Consistently, Hif-1 $\alpha$ protein in these organs, which is also deacetylated and stabilized by Sirt1, also increased in response to dehydration in the same four tissues of $X$. laevis (Figure 4.8B) (Joo et al., 2015; Wu et al., 2017). These results suggest that an increase in Sirtuin deacetylase activity in X. laevis may promote downstream PGC-1 $\alpha$ processes such as ureagenesis, but concurrently also suppress fatty acid oxidation by stabilizing Hif-1 $\alpha$. Indeed, ppara mRNA levels were suppressed in both liver and muscle during dehydration (Figure 4.9A). Together with decreased $h n f 4 a$ and $h n f 3 b$, a decrease in ppara mediated by Hif- $1 \alpha$ is evidence to suggest that fatty acid oxidation genes are suppressed during 
dehydration. Indeed, reductions in lcad and mcad mRNA levels were observed in the liver (Figure 4.9A) during dehydration, along with decreases in muscle $l c a d$, $m c a d$, and scad transcripts (Figure 4.9B).

Transcript levels of the cptla and cpt $1 b$ genes, which encode transport proteins that move fatty acids into the mitochondria, and thereby promote their catabolism, were either unchanged in the liver (Figure 4.11A) or increased in the muscle (Figure 4.11B) during dehydration. Reasons for these gene responses remain unclear at this point. However, these results demonstrate that the canonical suppression of fatty acid oxidation genes by interactions between Hif-1 $\alpha$ and ppar do not always occur as they were described in studies with cell lines. Although $\mathrm{pgclb}$ was also expected to be suppressed as a mechanism of downregulating fatty acid oxidation genes, it was found to increase in both liver and muscle during dehydration (Figure 4.9B). Interestingly, other studies have found that PGC-1 $\beta$ can activate angiogenesis (Rowe et al., 2011), a process that could be triggered by $p g c 1 b$ transcription in response to dehydration-mediated impairment of circulation.

In the heart, there was no observed change in ppara or $p g c 1 b$ gene transcripts during dehydration (Figure 4.9). However, there were increases in transcripts of fatty acid metabolism genes ( $m c a d, s c a d$, and cpt $1 \mathrm{~b}$ ) in medium dehydration, all three of which either returned to control levels or decreased relative to controls during high dehydration (Figures 4.10C and Figure 4.11C). These results show that the $X$. laevis heart undergoes significant metabolic remodelling during dehydration. Given that $X$. laevis heart rates increase during dehydration (Hillman, 1978), these observed increases in genes during medium dehydration may be an attempt to meet increased energy demands, as the heart 
relies primarily on fatty acids as a source of fuel. However, further studies are required to better understand the state of the $X$. laevis heart during dehydration.

\section{$\underline{4.4 .4 ~ H i f-1 \alpha \text { and glycolytic genes }}$}

Given that fatty acid oxidation appears to be negatively regulated at the gene expression level, targets were further studied in order to explore whether genes related to glucose metabolism were upregulated during dehydration. Indeed, although increased Hif- $1 \alpha$ can result in suppressed fatty acid oxidation, which appears to be the case from the results in the present chapter, it is also known that Hif-1 $\alpha$ also promotes the expression of genes that would enhance glycolytic capacity (Huang et al., 2014; Semenza, 2004). In this chapter, relative mRNA levels of glut4, $h k, p f k$, $p d k 1, p d k 3$, and $p d k 4$ were measured in the liver, muscle, and heart of dehydrated $X$. laevis. These are all known to be targets of HIF-1 $\alpha$ action. Increased levels of liver $h k$ mRNA were observed during dehydration, which, together with decreased transcript levels of fatty acid oxidation targets, suggest that the liver is switching towards anaerobic glycolysis (Figure 4.12). This is further supported by increases in $p d k 3$ and $p d k 4$ in the liver and muscle during dehydration. In conjunction with an increase in $h k$, an increase in transcript levels of these $p d k$ genes in the liver during dehydration suggests that pyruvate dehydrogenase activity is suppressed by inhibitory phosphorylation. Certainly, such a remodeling of metabolism would logically occur during dehydration when oxygen delivery to the cells is impaired. 
Unlike the liver in which an increase in $h k$ mRNA levels was observed, transcript levels of $h k$ and $p f k$ decreased in the skeletal muscle during dehydration (Figure 4.13). This difference between the two tissues suggest that while fatty acid oxidation may be suppressed in favour of glucose metabolism in the liver, both modes of fuel consumption may be lowered in the muscle. This may be a response whereby the animal is prioritizing its vital organs, such as the liver, over tissues such as the skeletal muscles. Indeed, it is known that blood circulation in dehydrating $X$. laevis is reduced to the skeletal muscles, but sustained in the vital organs (Hillman and Sommerfeldt, 1981), an exemplary response by $X$. laevis to prioritize vital organs over the skeletal muscle tissues.

The heart has an interesting response that included an increase in pdk4 mRNA during dehydration that supports the notion of anaerobic glycolysis, but was also accompanied by decreases in glut4, $h k$, and $p f k$ (Figure 4.14). Together, the results from the heart suggest the suppression of both glucose utilization and fatty acid oxidation in high dehydration. Although all animals under high dehydration had noticeable heartbeats when tissues were sampled, it can be speculated that broad decreases in metabolic gene expression is a sign of heart failure, particularly as the frogs continue to raise their heart rates while reaching their maximum dehydration tolerance (Hillman, 1978).

\section{$\underline{4.4 .5}$ Regulation of vegf by PGC-1 $\alpha$ and Hif- $1 \alpha$}

In addition to the regulation of fatty acid and glucose metabolism, PGC-1 $\alpha$ and Hif- $1 \alpha$ are transcription factors that are known to induce the expression of vegf that encodes vascular endothelial growth factor, a protein that activates angiogenesis (Arany et al., 2008; Joo et al., 2015; Shoag and Arany, 2010). Furthermore, levels of pgclb mRNA also increased in the liver and muscle during dehydration (Figure 4.9B) and PGC- 
$1 \beta$ is a known promoter of vegf expression in cell lines (Rowe et al., 2011). In order to explore whether or not these transcriptional regulators are activating angiogenesis during $X$. laevis dehydration, relative vegf mRNA levels were measured in 6 tissues. Transcript levels of vegf were increased in the liver during high dehydration, which is consistent with observed increases in pgcla, hifla, and pgclb (Figure 4.15). Together, the data suggest that metabolic remodelling in the liver is also accompanied by increased angiogenesis via upregulation of vegf, a response in which this vital organ attempts to cope with blood circulation that is impaired by dehydration-induced reductions of plasma volume. Despite increases in PGC-1 $\alpha$ mRNA and protein in the lungs (Chapter 2) and increases in Hif-1 $\alpha$ mRNA and protein in both the heart and lungs during dehydration (Figure 4.8), relative vegf mRNA levels were found to decrease in both tissues (Figure 4.15). As a result, it appears that the activation of vegf transcription is part of a broader dehydration response for the liver specifically, as opposed to other tissues analyzed in $X$. laevis.

\subsection{Conclusions}

The present chapter investigated the cellular pathways that have roles in metabolic control in $X$. laevis. The results suggested that MEF2C acts as an upstream regulator to PGC-1 $\alpha$ expression in the liver. Activation of PGC-1 $\alpha$ was expected and not surprising in the liver as it is an important molecular response by which ureagenesis is activated - a process that is known to occur in dehydrated $X$. laevis. However, PGC-1 $\alpha$ is also a known activator of fatty acid oxidation, a process that is not expected to occur during dehydration. The results revealed that $X$. laevis may be suppressing fatty acid

oxidation by increasing Hif-1 $\alpha$ levels in the liver. Indeed, genes that encode for acyl-CoA 
dehydrogenase enzymes were found to decrease in liver of dehydrating frogs.

Furthermore, results also revealed that Hif- $1 \alpha$ may be inducing the expression of genes that include glycolytic enzymes and pyruvate dehydrogenase kinases. Results were similar for the muscle, where not only fatty acid oxidation was suppressed, but glycolytic genes and pyruvate dehydrogenase kinases were also. In the heart, results suggested that fatty acid oxidation actually increases under medium dehydration - perhaps as a response to match increased energy demands. However, both glucose and fatty acid metabolism genes became downregulated under high dehydration conditions, when animals reached near-maximum tolerance to dehydration. Overall, the present chapter provided evidence about the modulations of cellular pathways that reprogram metabolic pathways during $X$. laevis dehydration. 


\subsection{References}

Arany, Z., Foo, S.-Y., Ma, Y., Ruas, J.L., Bommi-Reddy, A., Girnun, G., Cooper, M., Laznik, D., Chinsomboon, J., Rangwala, S.M., Baek, K.H., Rosenzweig, A., Spiegelman, B.M., 2008. HIF-independent regulation of VEGF and angiogenesis by the transcriptional coactivator PGC-1 $\alpha$. Nature 451, 1008-1012.

Balinsky, J.B., Choritz, E.L., Coe, C.G., van der Schans, G.S., 1967. Amino acid metabolism and urea synthesis in naturally aestivating Xenopus laevis. Comp. Biochem. Physiol. 22, 59-68.

Balinsky, J.B., Cragg, M.M., Baldwin, E., 1961. The adaptation of amphibian waste nitrogen excretion to dehydration. Comp. Biochem. Physiol. 3, 236-244.

Chen, Z., Tang, N., Wang, X., Chen, Y., 2017. The activity of the carbamoyl phosphate synthase 1 promoter in human liver-derived cells is dependent on hepatocyte nuclear factor 3-beta. J. Cell. Mol. Med. 21, 2036-2045.

Czubryt, M.P., McAnally, J., Fishman, G.I., Olson, E.N., 2003. Regulation of peroxisome proliferator-activated receptor gamma coactivator 1 alpha (PGC-1 alpha ) and mitochondrial function by MEF2 and HDAC5. Proc. Natl. Acad. Sci. U. S. A. 100, 1711-6.

Fernandez-Marcos, P.J., Auwerx, J., 2011. Regulation of PGC-1 $\alpha$, a nodal regulator of mitochondrial biogenesis, in: American Journal of Clinical Nutrition. p. 884S-90.

Hallows, W.C., Yu, W., Smith, B.C., Devires, M.K., Ellinger, J.J., Someya, S., Shortreed, M.R., Prolla, T., Markley, J.L., Smith, L.M., Zhao, S., Guan, K.L., Denu, J.M., 2011. Sirt3 Promotes the Urea Cycle and Fatty Acid Oxidation during Dietary Restriction. Mol. Cell 41, 139-149.

Handschin, C., Rhee, J., Lin, J., Tarr, P.T., Spiegelman, B.M., 2003. An autoregulatory loop controls peroxisome proliferator-activated receptor coactivator 1 expression in muscle. Proc. Natl. Acad. Sci. 100, 7111-7116.

Hillman, S.S., 1978. The roles of oxygen delivery and electrolyte levels in the dehydrational death of Xenopus laevis. J. Comp. Physiol. B 128, 169-175.

Hillman, S.S., Sommerfeldt, R.W., 1981. Microsphere studies of amphibian systemic blood flow redistribution during dehydration, hypovolemia, and salt load. J. Exp. Zool. 218, 305-308.

Huang, D., Li, T., Li, X., Zhang, L., Sun, L., He, X., Zhong, X., Jia, D., Song, L., Semenza, G.L., Gao, P., Zhang, H., 2014. HIF-1-mediated suppression of acyl-CoA dehydrogenases and fatty acid oxidation is critical for cancer progression. Cell Rep. 8, 1930-1942.

Inoue, Y., Hayhurst, G.P., Inoue, J., Mori, M., Gonzalez, F.J., 2002. Defective ureagenesis in mice carrying a liver-specific disruption of hepatocyte nuclear factor $4 \alpha(\mathrm{HNF} 4 \alpha)$ : HNF4 $\alpha$ regulates ornithine transcarbamylase in vivo. J. Biol. Chem. 
$277,25257-25265$.

Joo, H.Y., Yun, M., Jeong, J., Park, E.R., Shin, H.J., Woo, S.R., Jung, J.K., Kim, Y.M., Park, J.J., Kim, J., Lee, K.H., 2015. SIRT1 deacetylates and stabilizes hypoxiainducible factor- $1 \alpha(\mathrm{HIF}-1 \alpha)$ via direct interactions during hypoxia. Biochem. Biophys. Res. Commun. 462, 294-300.

Laurent, G., de Boer, V.C.J., Finley, L.W.S., Sweeney, M., Lu, H., Schug, T.T., Cen, Y., Jeong, S.M., Li, X., Sauve, A.A., Haigis, M.C., 2013. SIRT4 Represses Peroxisome Proliferator-Activated Receptor $\alpha$ Activity To Suppress Hepatic Fat Oxidation. Mol. Cell. Biol. 33, 4552-4561.

Li, L., Zhang, P., Bao, Z., Wang, T., Liu, S., Huang, F., 2016. PGC-1 $\alpha$ Promotes Ureagenesis in Mouse Periportal Hepatocytes through SIRT3 and SIRT5 in Response to Glucagon. Sci. Rep. 6, 24156.

Lovekamp-Swan, T., Jetten, A.M., Davis, B.J., 2003. Dual activation of PPARalpha and PPARgamma by mono-(2-ethylhexyl) phthalate in rat ovarian granulosa cells. Mol. Cell. Endocrinol. 201, 133-141.

Malik, A.I., Storey, K.B., 2011. Transcriptional regulation of antioxidant enzymes by FoxO1 under dehydration stress. Gene 485, 114-119.

Mandard, S., Müller, M., Kersten, S., 2004. Peroxisome proliferator-activated receptor a target genes. Cell. Mol. Life Sci. 61, 393-416.

Martinez-Jimenez, C.P., Kyrmizi, I., Cardot, P., Gonzalez, F.J., Talianidis, I., 2010. Hepatocyte nuclear factor 4alpha coordinates a transcription factor network regulating hepatic fatty acid metabolism. Mol. Cell. Biol. 30, 565-77.

Nakagawa, T., Lomb, D.J., Haigis, M.C., Guarente, L., 2009. SIRT5 Deacetylates Carbamoyl Phosphate Synthetase 1 and Regulates the Urea Cycle. Cell 137, 560570 .

Nasrin, N., Wu, X., Fortier, E., Feng, Y., Baré, O.C., Chen, S., Ren, X., Wu, Z., Streeper, R.S., Bordone, L., 2010. SIRT4 regulates fatty acid oxidation and mitochondrial gene expression in liver and muscle cells. J. Biol. Chem. 285, 31995-32002.

Olmos, Y., Sánchez-Gómez, F.J., Wild, B., García-Quintans, N., Cabezudo, S., Lamas, S., Monsalve, M., 2013. SirT1 Regulation of Antioxidant Genes Is Dependent on the Formation of a FoxO3a/PGC-1 $\alpha$ Complex. Antioxid. Redox Signal. 19, 1507-1521.

Purushotham, A., Schug, T.T., Xu, Q., Surapureddi, S., Guo, X., Li, X., 2009.

Hepatocyte-Specific Deletion of SIRT1 Alters Fatty Acid Metabolism and Results in Hepatic Steatosis and Inflammation. Cell Metab. 9, 327-338.

Rodgers, J.T., Lerin, C., Haas, W., Gygi, S.P., Spiegelman, B.M., Puigserver, P., 2005. Nutrient control of glucose homeostasis through a complex of PGC-1 $\alpha$ and SIRT1. Nature 434, 113-118. 
Rowe, G.C., Jang, C., Patten, I.S., Arany, Z., 2011. PGC-1 $\beta$ regulates angiogenesis in skeletal muscle. AJP Endocrinol. Metab. 301, E155-E163.

Semenza, G.L., 2004. Hydroxylation of HIF-1: Oxygen Sensing at the Molecular Level. Physiology 19, 176-182.

Shoag, J., Arany, Z., 2010. Regulation of hypoxia-inducible genes by PGC-1 $\alpha$. Arterioscler. Thromb. Vasc. Biol.

Wolfrum, C., Stoffel, M., 2006. Coactivation of Foxa2 through Pgc-1 $\beta$ promotes liver fatty acid oxidation and triglyceride/VLDL secretion. Cell Metab. 3, 99-110.

Wu, C.-W., Tessier, S.N., Storey, K.B., 2017. Regulation of the insulin-Akt signaling pathway and glycolysis during dehydration stress in the African clawed frog Xenopus laevis. Biochem. Cell Biol. 95, 663-671. 


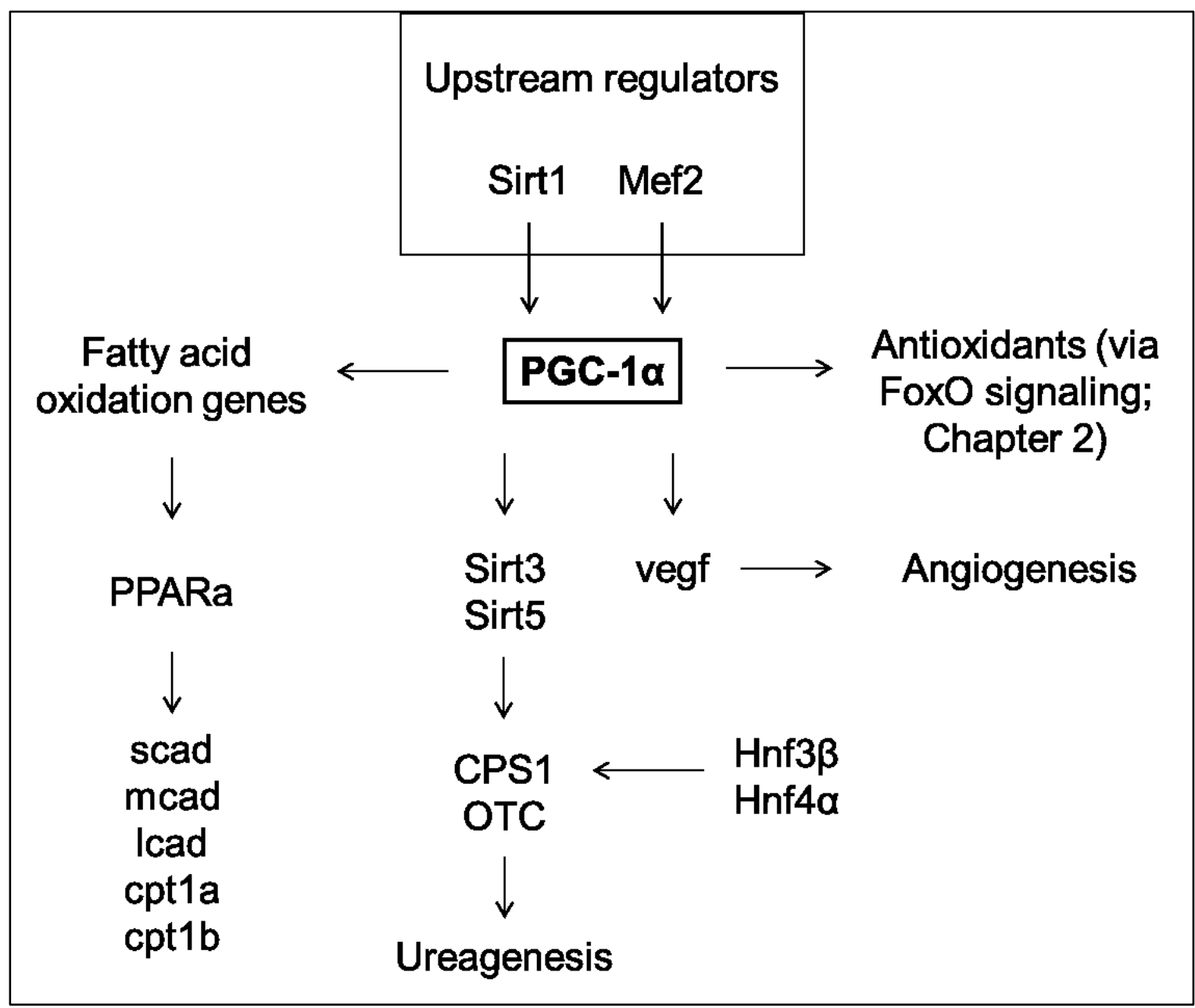

Figure 4.1. The "canonical" PGC- $1 \alpha$ pathway. The expression and activity of PGC- $1 \alpha$ is known to be increased by Sirt and MEF2 upstream regulators. Once activated, PGC-1 $\alpha$ plays diverse cellular roles including in nitrogen and fatty acid metabolism, in addition to the regulation of antioxidants and angiogenesis. 


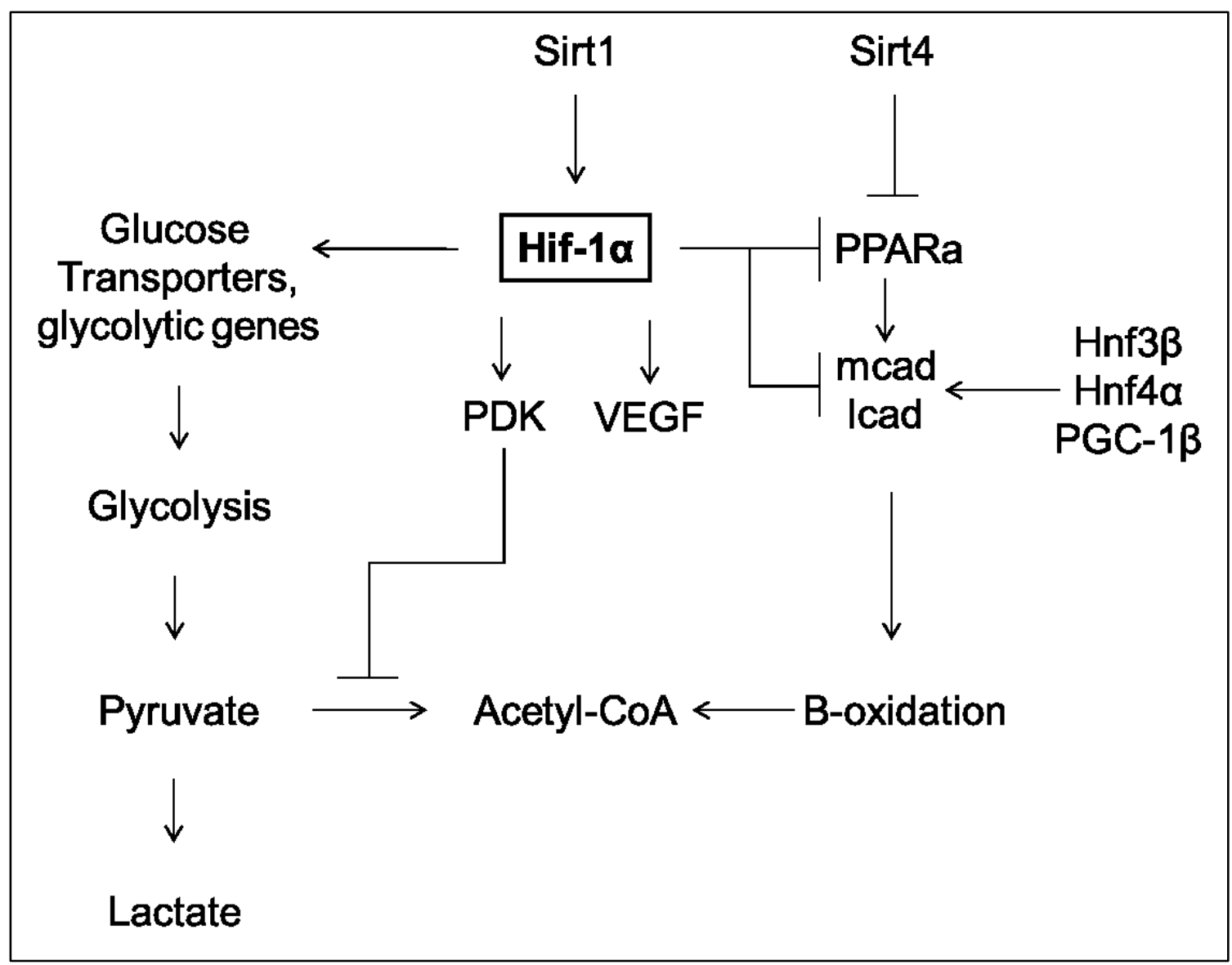

Figure 4.2. The "canonical" Hif-1a pathway. The Hif-1a transcription factor, similar to PGC-1 $\alpha$, is stabilized and inducible by Sirt deacetylase activity, and also regulates angiogenic genes. Unlike PGC-1 $\alpha$, Hif-1a suppresses fatty acid oxidation in favour of glucose metabolism. 

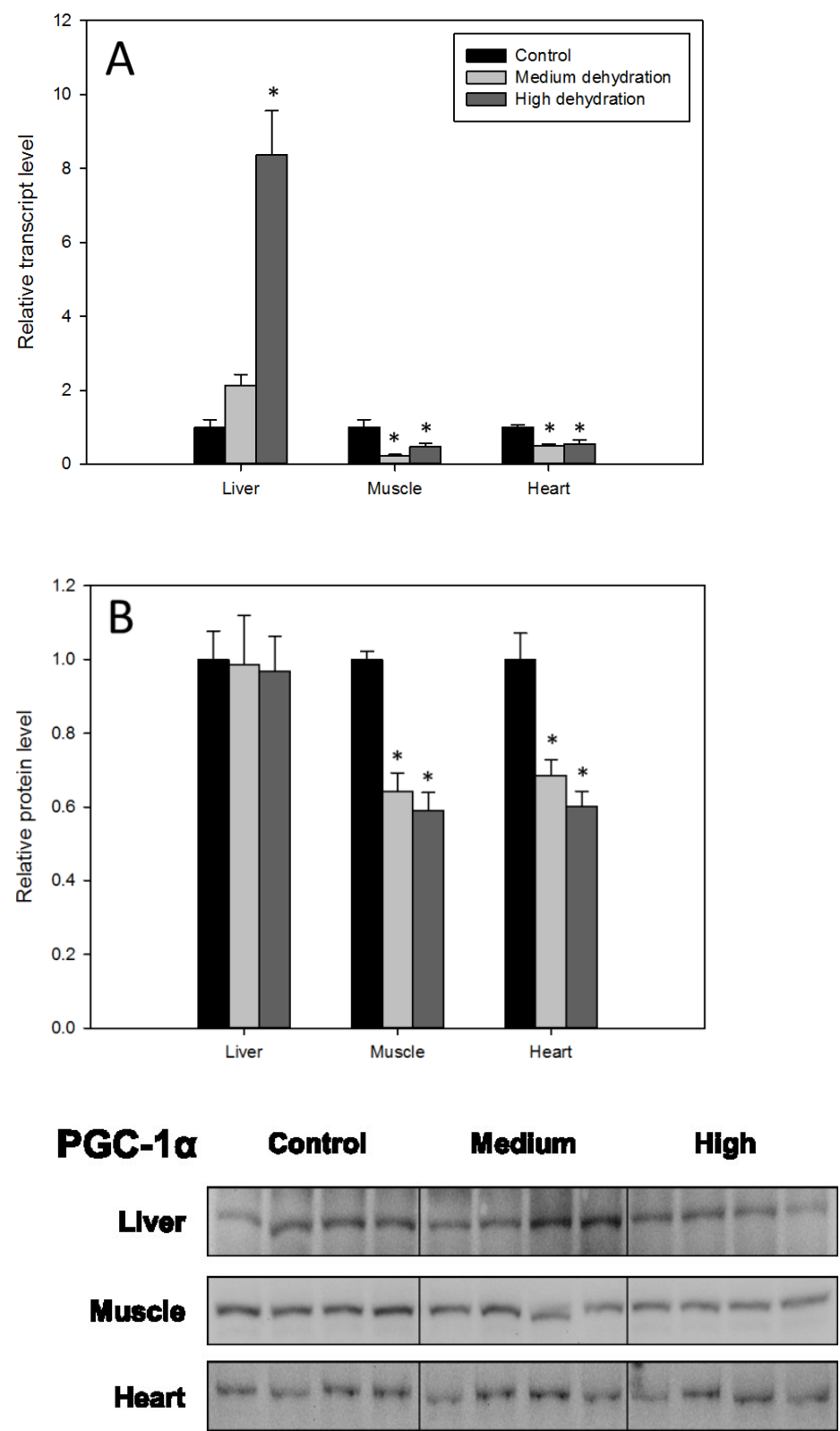

Figure 4.3. Relative pgcla mRNA (A) and PGC-1 $\alpha$ protein levels (B) determined by RTqPCR and immunoblotting, respectively in the liver, muscle, and heart of $X$. laevis under control, medium, and high levels of whole-body dehydration. Gene expression data was quantified with RT-qPCR and standardized against rpl27 as the reference gene for liver and muscle, whereas gapdh was used for heart. Chemiluminescent data from immunoblots was standardized against total protein loaded as determined by subsequent Coomassie staining of membranes. Histograms show means \pm SEM, $n=4$ independent biological replicates. Statistically significant differences compared to the control condition are denoted with an asterisk $(*)$ and were determined with a one-way ANOVA and post hoc Dunnett's test $(p<0.05)$. 

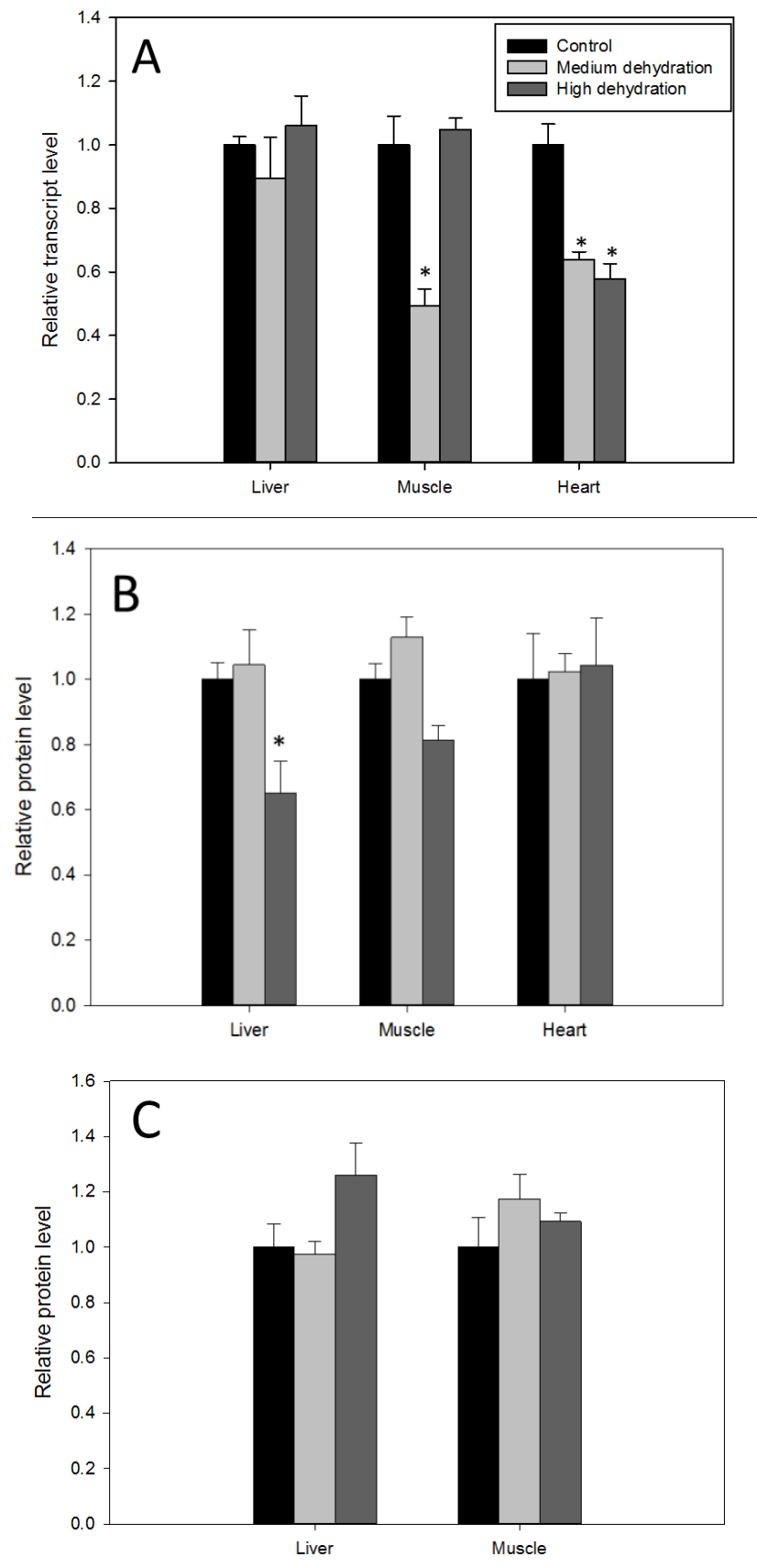

Figure 4.4. Relative mef $2 a$ mRNA (A), MEF2A protein (B), and MEF2A nuclear protein levels determined by RT-qPCR and immunoblotting, respectively in the liver, muscle, and heart of $X$. laevis under control, medium, and high levels of whole-body dehydration. Other information as in Figure 4.3; immunoblot images are shown in Figure 4.6. 

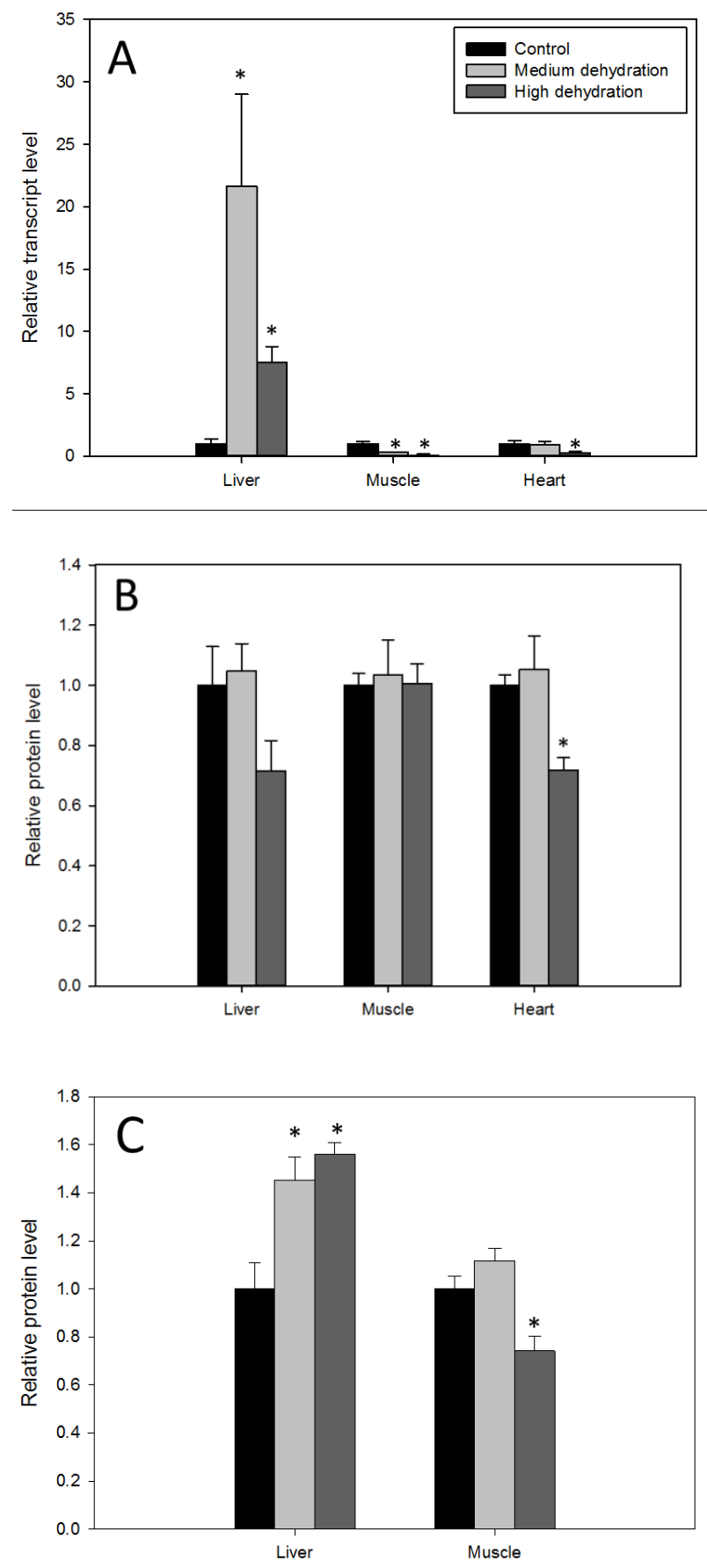

Figure 4.5. Relative mef $2 c$ mRNA (A), MEF2C protein (B), and MEF2C nuclear protein levels determined by RT-qPCR and immunoblotting, respectively in the liver, muscle, and heart of $X$. laevis under control, medium, and high levels of whole-body dehydration. Other information as in Figure 4.3; immunoblot images are shown in Figure 4.6. 


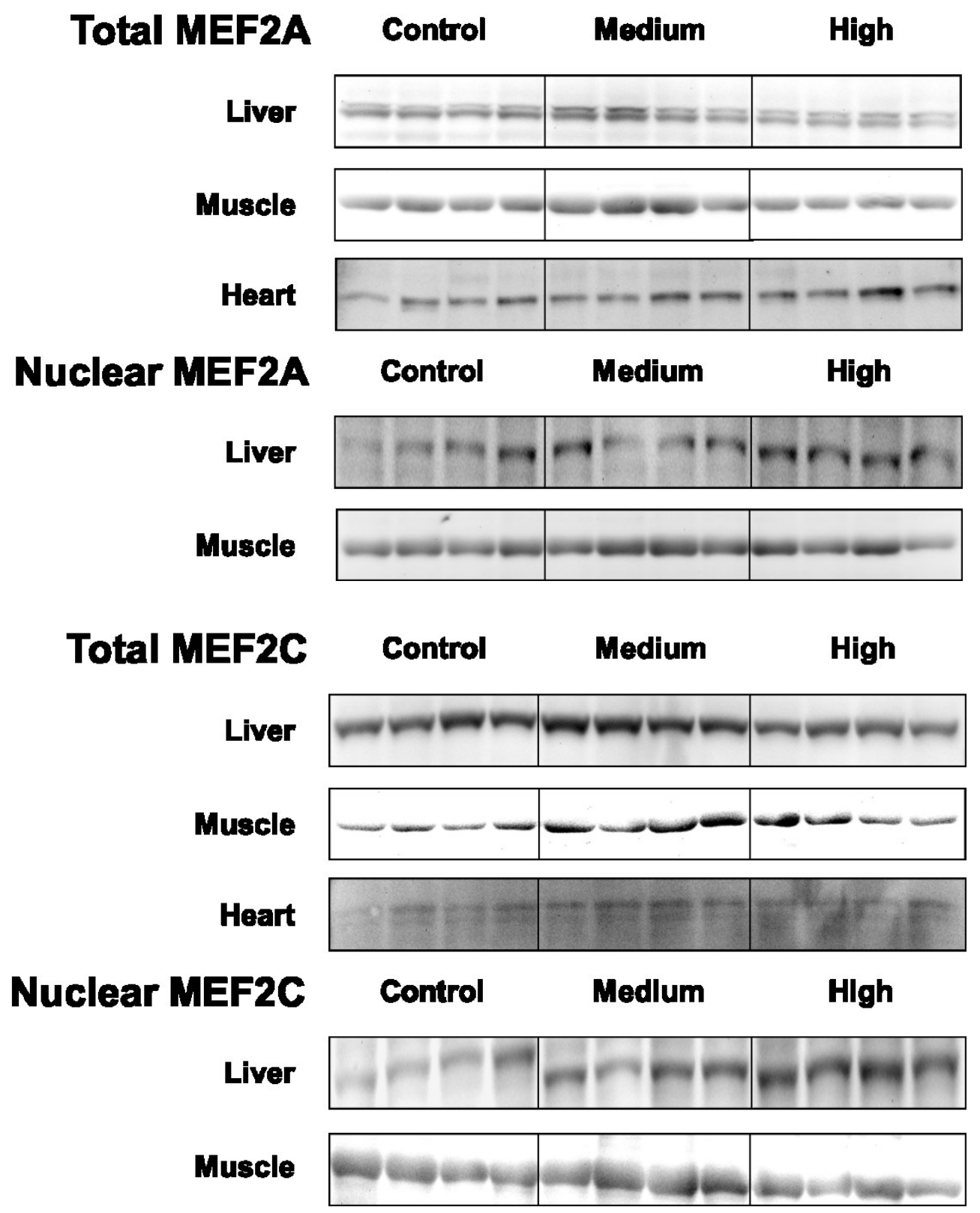

Figure 4.6. Visualization of chemiluminescent bands of immunoblots for MEF2A and MEF2C proteins used to provide quantitative data in Figures 4.4 and 4.5. 


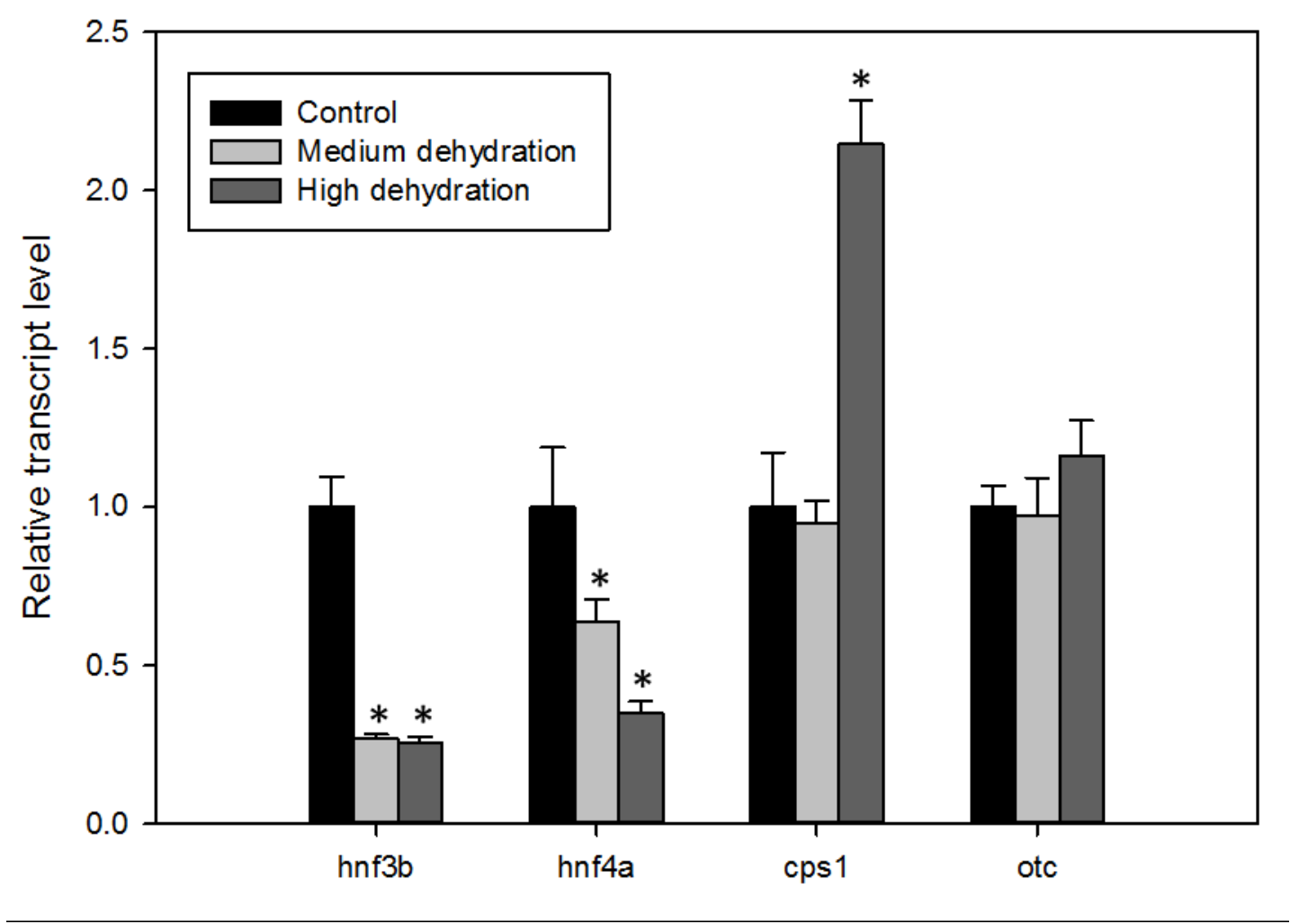

Figure 4.7. Relative mRNA levels of hepatic transcription factors $h n f 3 b$ and $h n f 4 a$ and urea cycle genes cps 1 and otc, as determined by RT-qPCR, in the liver of $X$. laevis under control, medium, and high levels of whole-body dehydration. Other information as in Figure 4.3. 

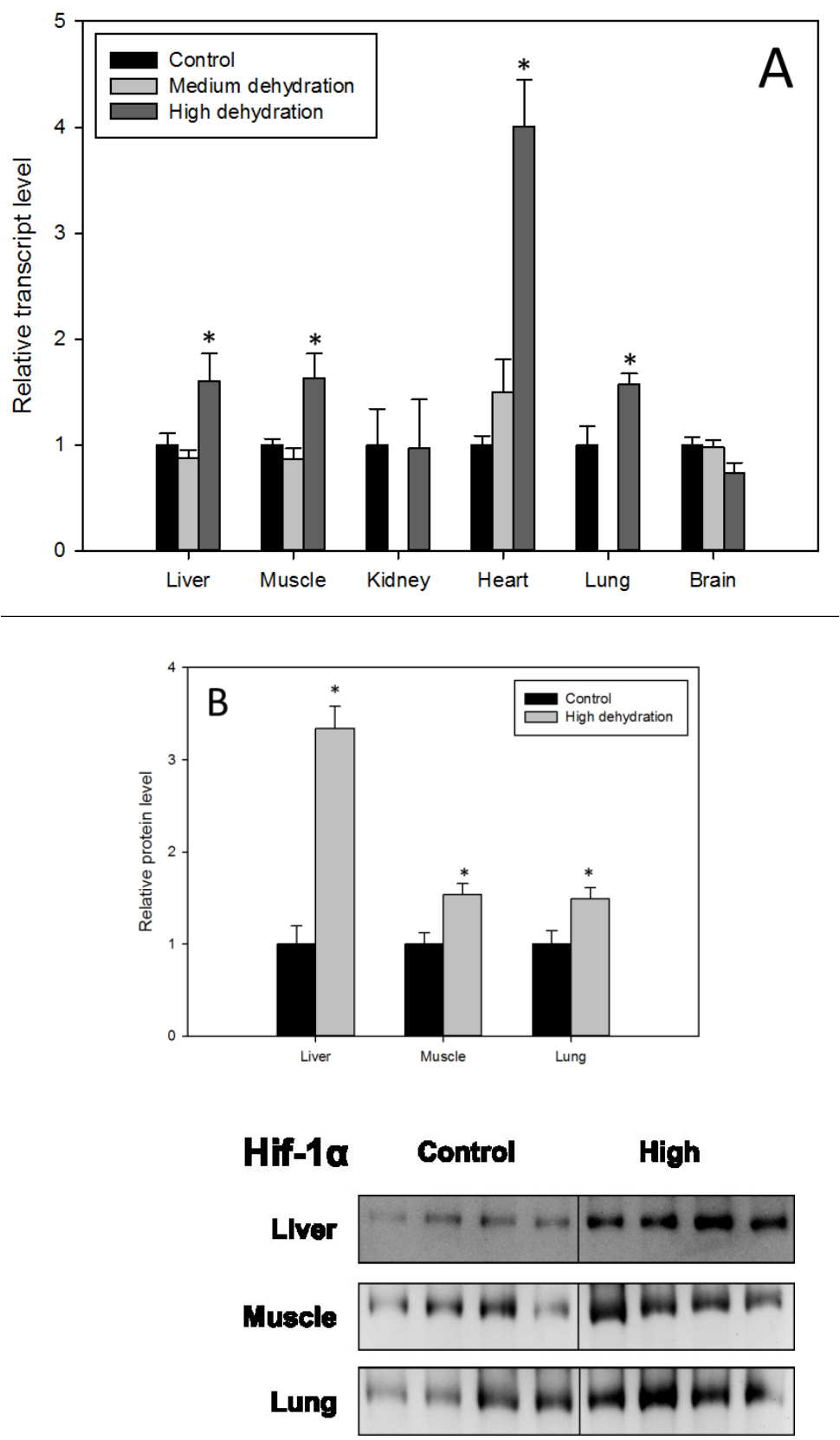

Figure 4.8. Relative hifla mRNA (A) and Hif-1 $\alpha$ protein levels (B) determined by RTqPCR and immunoblotting, respectively, in 6 tissues of $X$. laevis under control, medium, or high levels of whole-body dehydration. Gene expression data was quantified with RTqPCR and standardized against $r p l 27$ as the reference gene for all tissues except the heart, where gapdh was used. Histograms show means \pm SEM, $n=4$ independent biological replicates for all tissues except for kidney and lung RT-qPCR analyses, which are $n=8$ and $n=6$, respectively. Other information as in Figure 4.3.

*Heart, brain and kidney Hif-1 $\alpha$ protein levels were previously measured in dehydrated $X$. laevis (Wu et al., 2017) and only heart was sensitive to dehydration (upregulation)* 

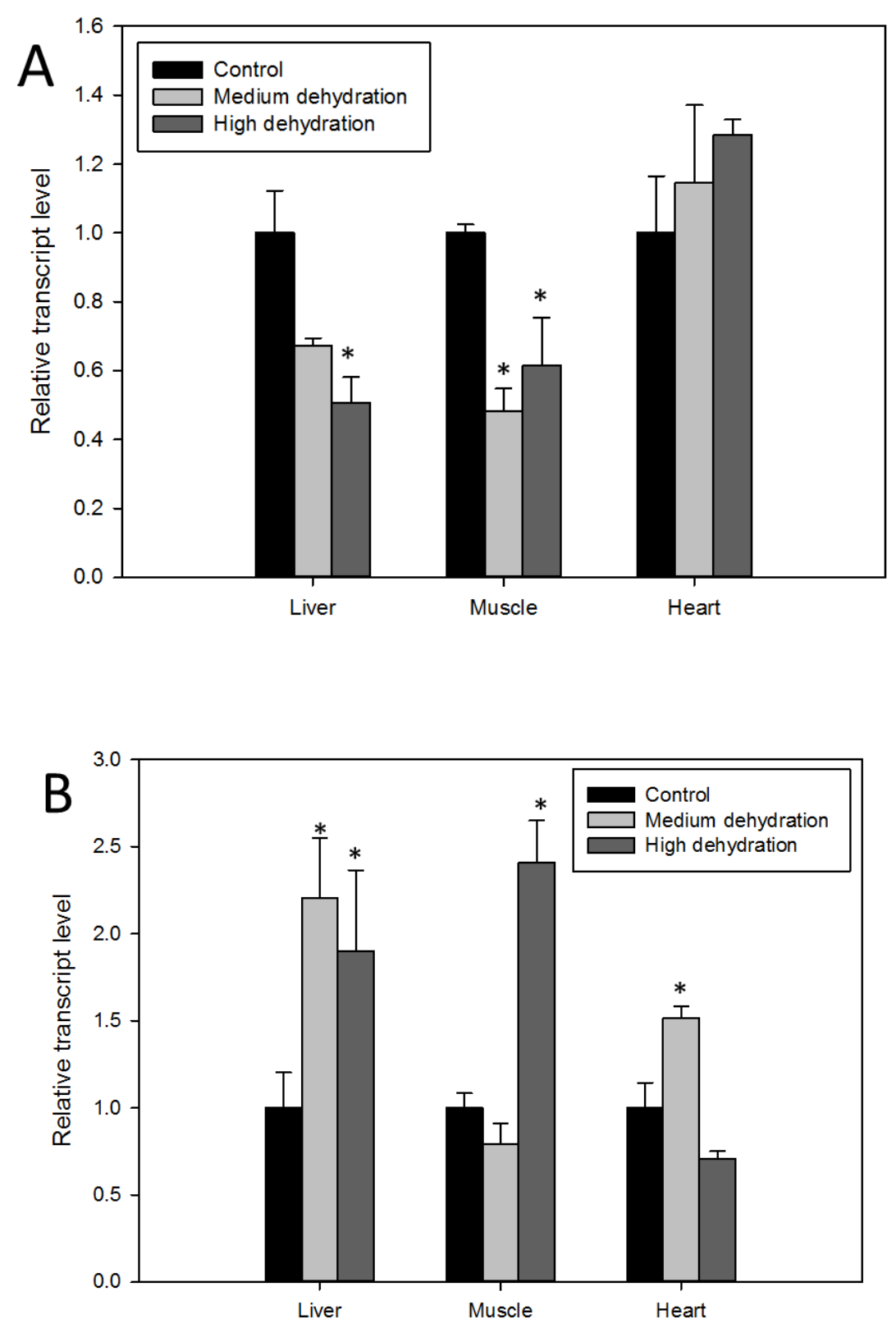

Figure 4.9. Relative mRNA levels of ppara (A) and pgclb (B) as determined by RTqPCR, in the liver, muscle, and heart of $X$. laevis under control, medium, and high levels of whole-body dehydration. Other information as in Figure 4.3. 

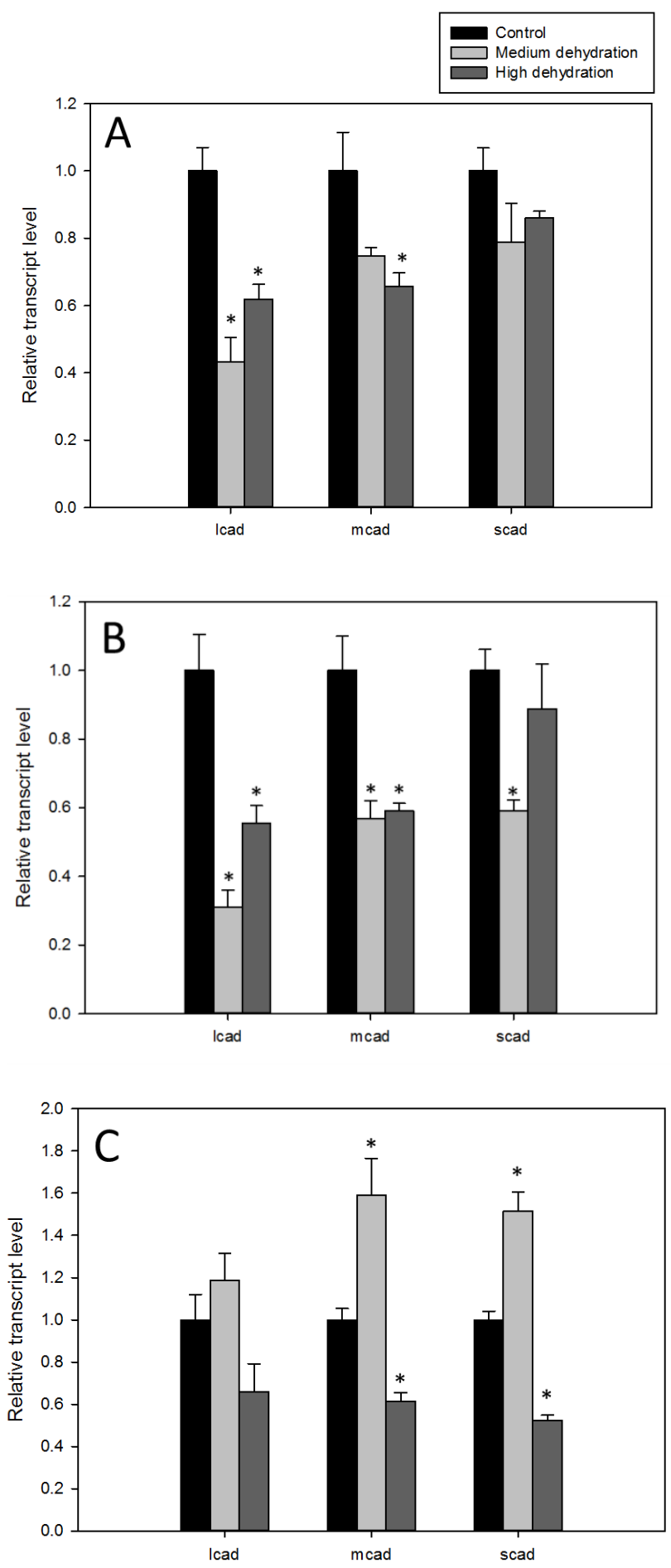

Figure 4.10. Relative mRNA levels of $l c a d$, $m c a d$, and scad as determined by RT-qPCR, in the liver (A), muscle (B), and heart (C) of X. laevis under control, medium, and high levels of whole-body dehydration. Other information as in Figure 4.3. 

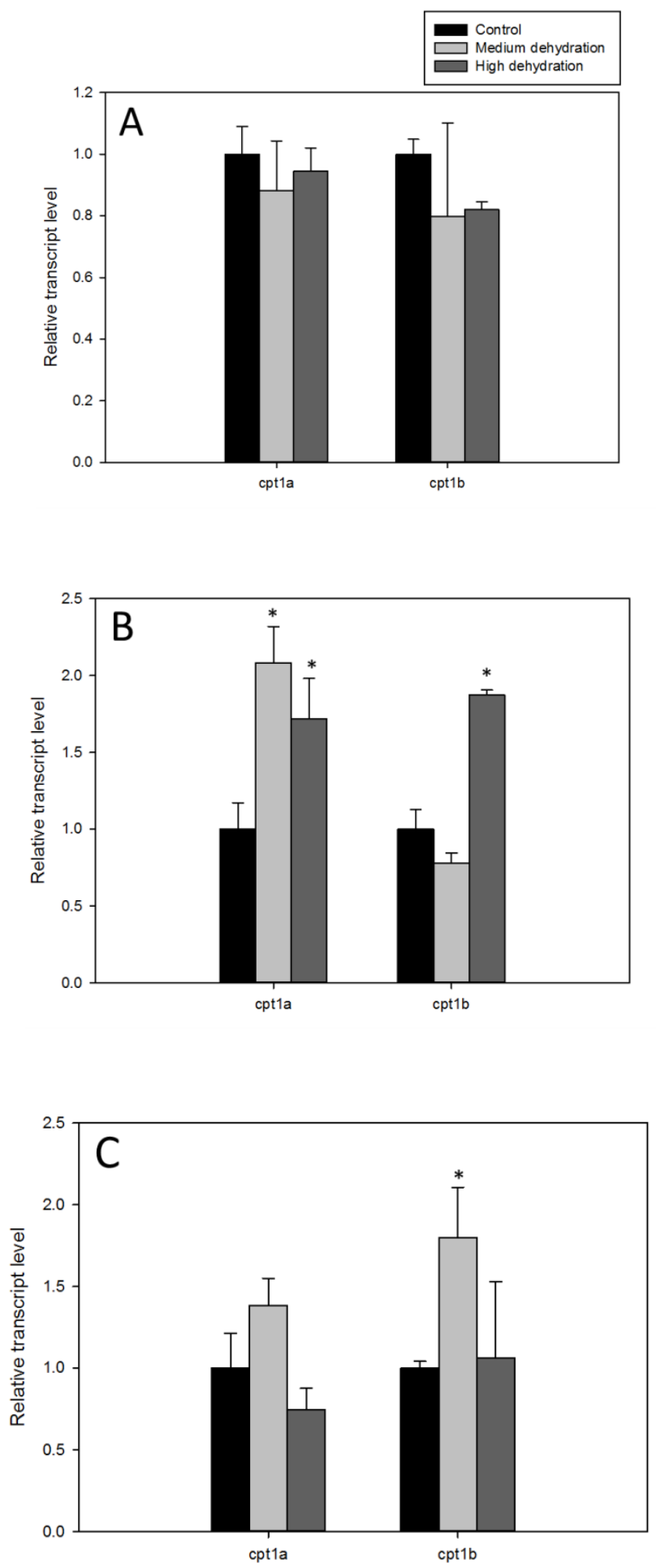

Figure 4.11. Relative mRNA levels of $c p t 1 a$ and $c p t 1 b$ as determined by RT-qPCR, in the liver (A), muscle (B), and heart (C) of $X$. laevis under control, medium, and high levels of whole-body dehydration. Other information as in Figure 4.3. 


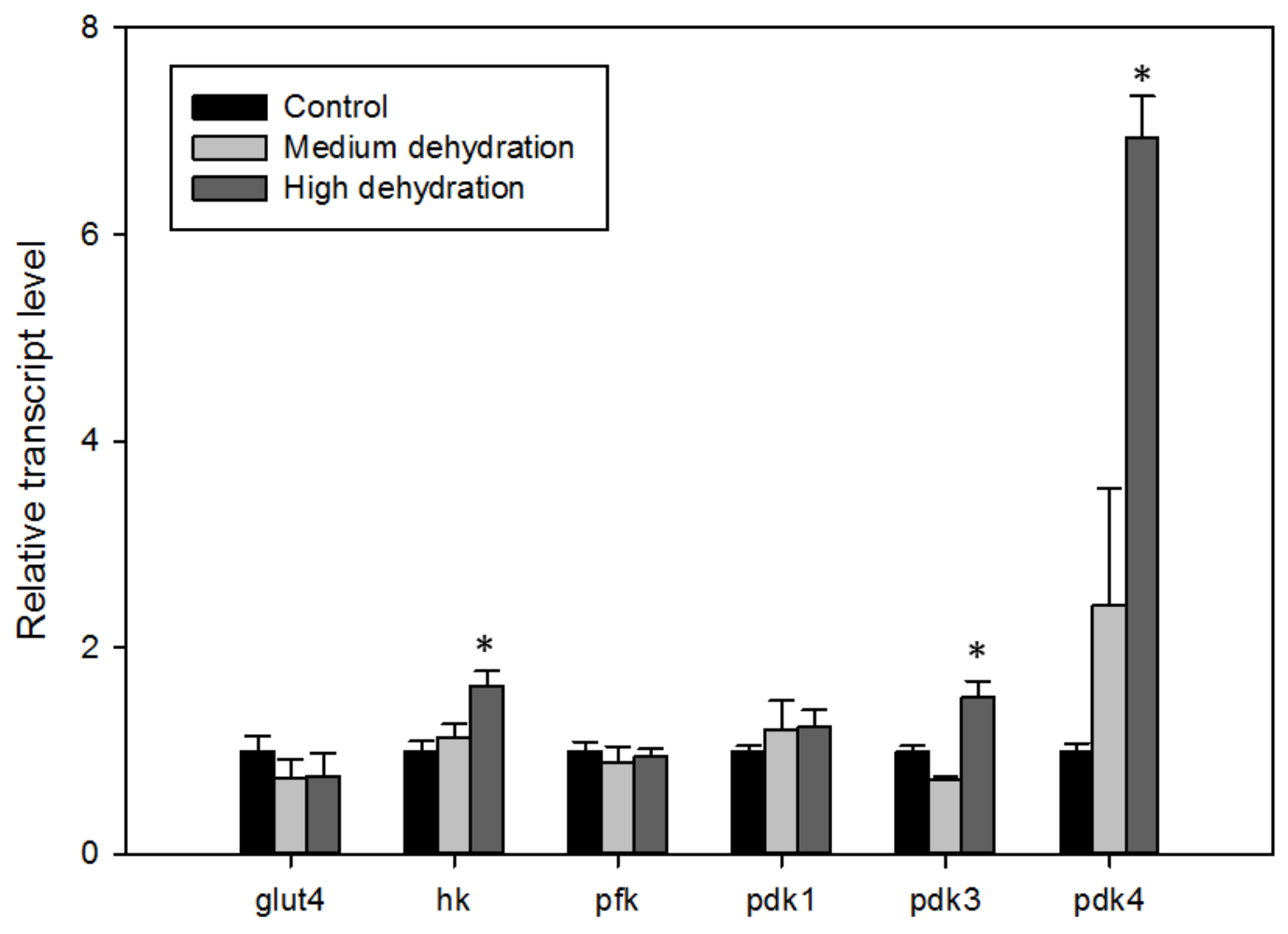

Figure 4.12. Relative mRNA levels of glut4, $h k, p f k, p d k 1, p d k 3$ and $p d k 4$ as determined by RT-qPCR, in the liver of $X$. laevis under control, medium, and high levels of wholebody dehydration. Other information as in Figure 4.3. 


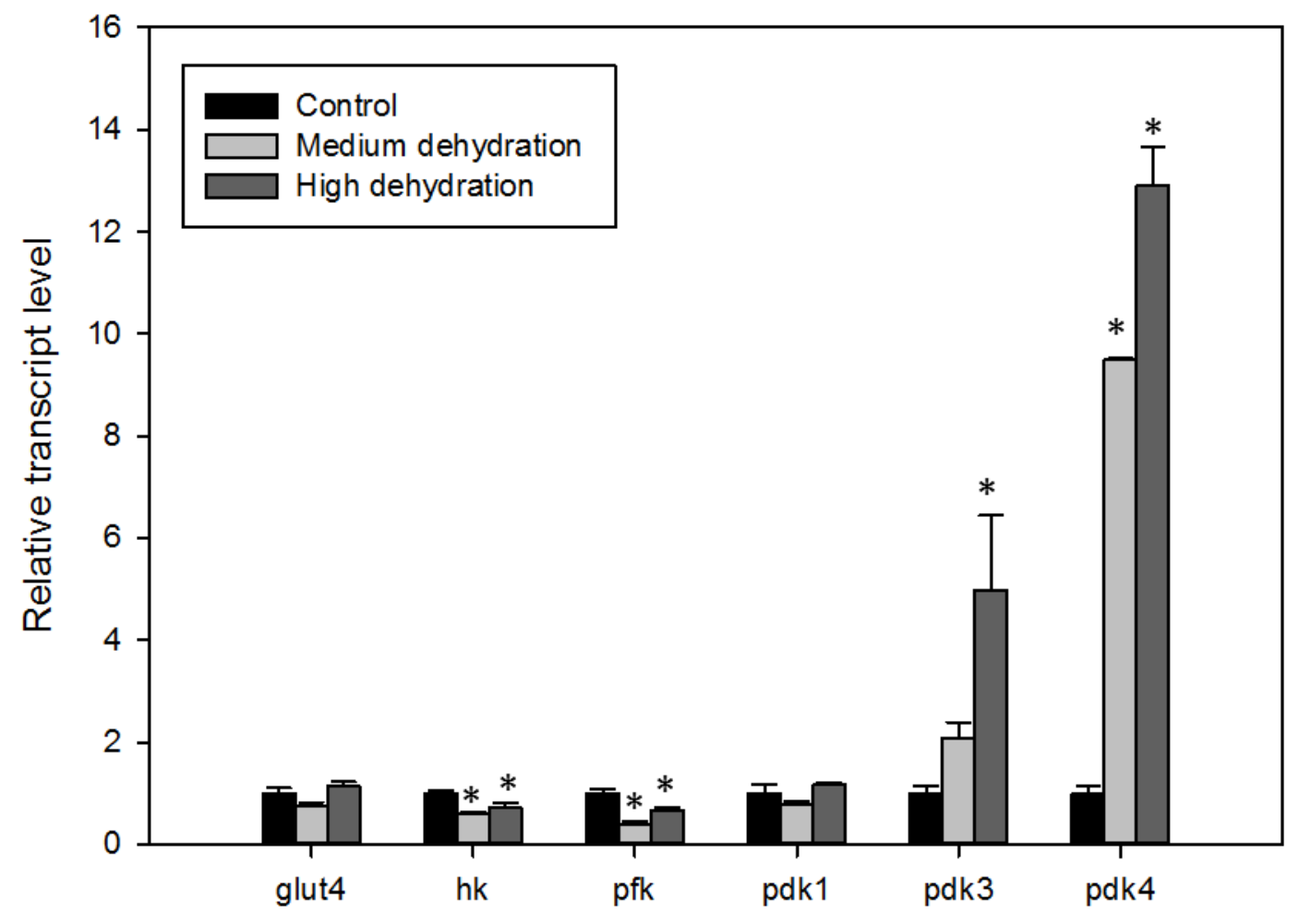

Figure 4.13. Relative mRNA levels of $g l u t 4, h k, p f k, p d k 1, p d k 3$ and $p d k 4$ as determined by RT-qPCR, in the muscle of X. laevis under control, medium, and high levels of wholebody dehydration. Other information as in Figure 4.3. 


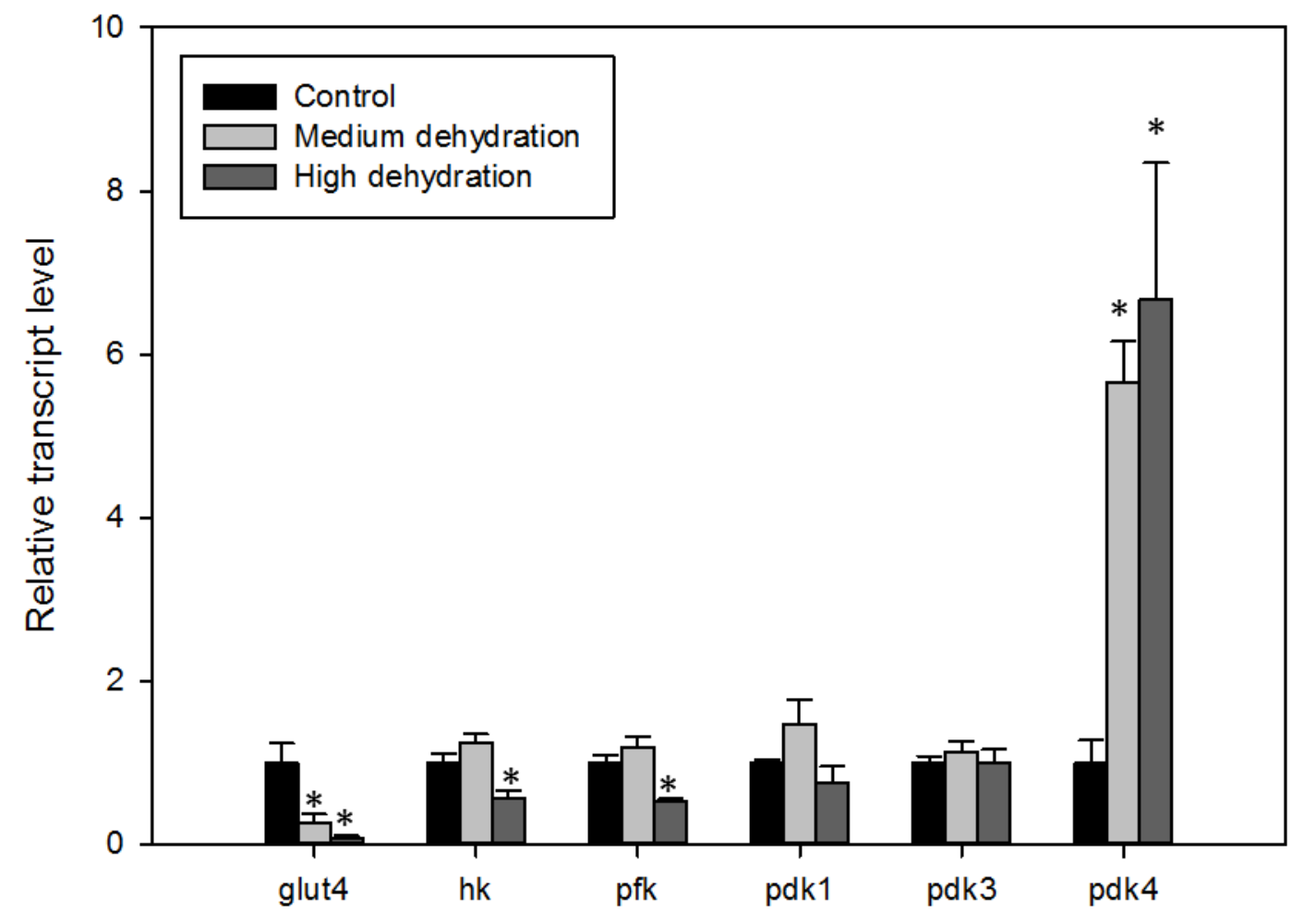

Figure 4.14. Relative mRNA levels of glut4, $h k, p f k, p d k 1, p d k 3$ and $p d k 4$ as determined by RT-qPCR, in the heart of $X$. laevis under control, medium, and high levels of wholebody dehydration. Other information as in Figure 4.3. 


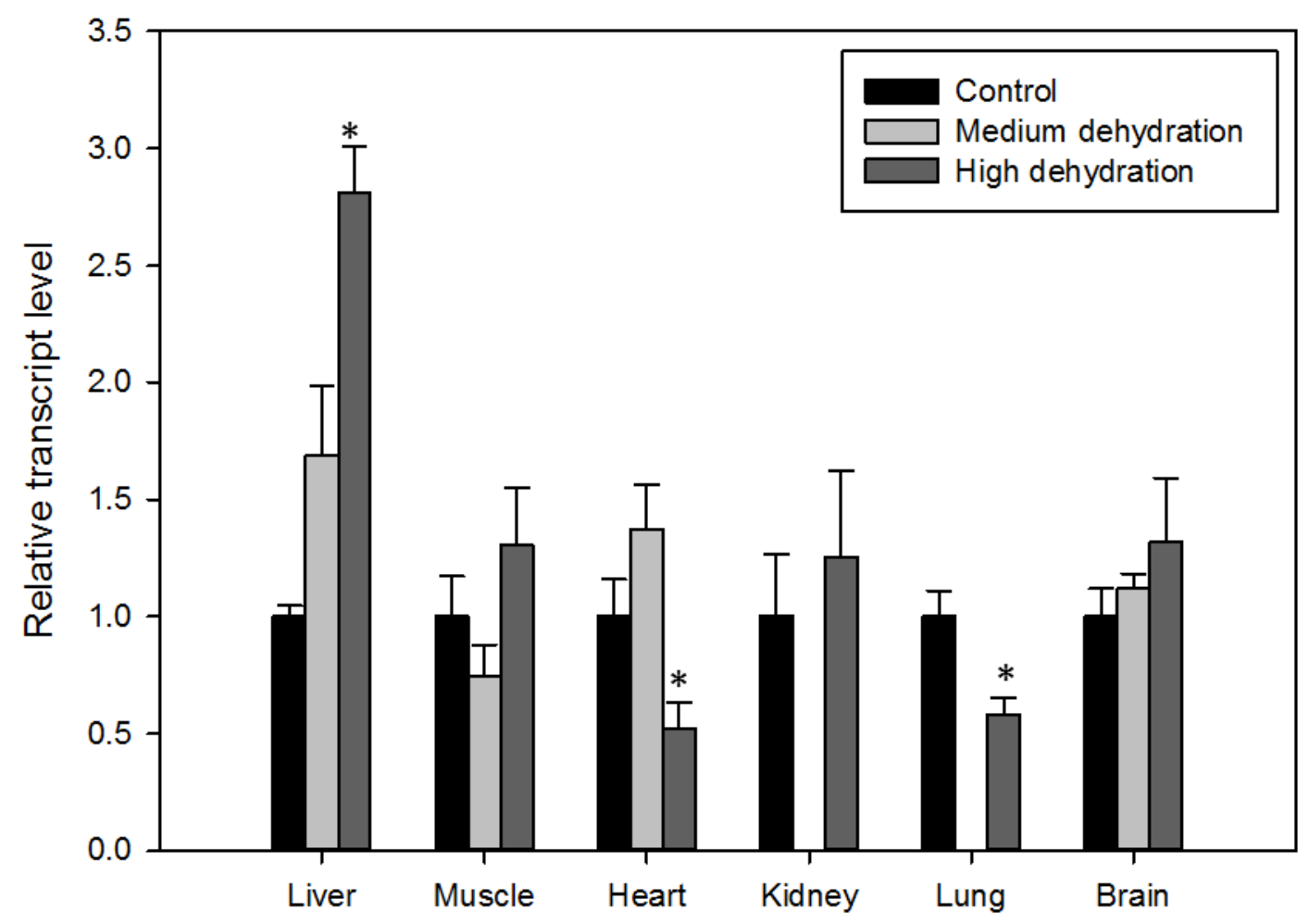

Figure 4.15. Relative mRNA levels of vegf as determined by RT-qPCR, in 6 tissues of $X$. laevis under control, medium, and high levels of whole-body dehydration. Other information as in Figure 4.8. 


\section{CHAPTER 5}

General Discussion 


\subsection{General Discussion}

Dehydration has been studied in various contexts (exercise in humans, drought response in plants, desiccation in tardigrades, etc). The physiological and behavioural effects of dehydration have been investigated, however, less so is known about the cellular responses that involve the regulation of gene and protein expression in particular for vertebrates. In the General Introduction (Chapter 1) of this thesis, a case was made for $X$. laevis as an ideal vertebrate model to study the cellular responses to whole-body dehydration. The use of such an amphibian is practical as it allows us to observe the effects of $\sim 30 \%$ loss of total body water. The African clawed frog, X. laevis, in particular, is one of the few amphibians that has had its genome annotated multiple times, allowing experiments that rely on sequence data to be performed confidently.

In this thesis, annotated genome resources are leveraged to accurately quantify gene mRNA levels of interest with RT-qPCR (Appendix C). Furthermore, as most commercially available antibodies are not designed for X. laevis in particular, the primary sequences of the protein targets made available from the annotated genome are used to accurately calculate the molecular weight of protein targets for Western immunoblot quantification (Appendix G). Certainly, other amphibians such as the North American wood frog, Rana sylvatica, are capable of tolerating much higher levels of dehydration, but the $R$. sylvatica genome has yet to be sequenced as of the writing of this thesis.

Unlike certain mammals and lower vertebrates that are capable of entering a state of hypometabolism when faced with severe environmental conditions, X. laevis in the context of this thesis (animals are exposed to dehydration as done previously by other groups (Hillman, 1978)) does not appear to do so, even when faced with near-maximum 
levels of whole-body dehydration. Indeed, it is important to clearly state the physiological conditions of $X$. laevis during whole-body dehydration before the cellular responses explored in this thesis can be discussed. When encountering dehydration, a decrease in plasma volume increases hematocrit and blood viscosity, which causes a restriction of oxygen delivery to the tissues. However, instead of entering a state of hypometabolism in which metabolic demands are lowered, resting heart rate is increased in order to compensate for the circulatory system, which becomes increasingly ineffective with dehydration (Hillman, 1978). Indeed, this behaviour of increasing heart rate in response to dehydration continues until the limits of dehydration tolerance are approached, at which time whole-animal lactate levels are observed to increase (Hillman, 1978).

Other studies that have explored the cellular and molecular responses to wholebody dehydration in $X$. laevis have provided evidence that reversible protein phosphorylation plays a major role in the dehydration response by modulating a broad range of processes including transcription factor binding and enzyme kinetic properties (Childers and Storey, 2016; Dawson et al., 2018; Katzenback et al., 2014; Malik and Storey, 2011, 2009). However, the presence of differential protein acetylation is relatively unexplored in the context of dehydrated $X$. laevis. In this thesis, a role for protein acetylation is investigated by characterizing the Sirt family, comprised of 7 members (Chapter 2). Each of these 7 members is known to play unique roles in various cellular processes that have been previously elucidated in other studies (Chen et al., 2011; Jacobs et al., 2008; Laurent et al., 2013; Liu et al., 2017; Nakagawa et al., 2009a; Olmos et al., 2013; Rodgers et al., 2008). Indeed, these processes include those that have been previously been shown to be regulated, or ones that are hypothesized to be regulated, in 
$X$. laevis exposed to whole-body dehydration, such as antioxidant upregulation, ureagenesis, angiogenesis, glucose metabolism, and fatty acid oxidation. The goal of the present thesis was to investigate the cellular responses to whole-body dehydration in $X$. laevis by examining three aspects: (1) Determination of the response of Sirts to wholebody dehydration at the mRNA and activity level, and whether or not this has an effect on known Sirt targets (Ac-SOD2), (2) Sirt-mediated antioxidant upregulation particularly through the Sirt/PGC-1 $\alpha /$ FoxO pathway, and (3) the regulation of metabolic processes by Sirt-regulated PGC-1 $\alpha$ and Hif- $1 \alpha$. Throughout this thesis, these three points were assessed at the mRNA transcription, total protein, protein posttranslational modification, and activity levels.

\subsection{The role of Sirtuin deacetylases during $X$. laevis dehydration}

In Chapter 2, relative transcript levels of the 7 sirt genes were quantified in the 6 tissues of control and dehydrated X. laevis. It was reasoned that if the Sirtuin deacetylases had a role in the broader dehydration response, then X. laevis may be observed to expend energy to actively increase mRNA abundance of these genes, with which more Sirt protein could be translated. The relative sirt mRNA levels were indeed found to be differentially regulated during dehydration, in a tissue-specific manner. Increases in sirt 1 (liver and lung), sirt2 (lung and skeletal muscle), sirt3 (skeletal muscle), and sirt6 (lung) were found in response to dehydration (Table 2.1). Furthermore, increases in global Sirt activity corresponded with these increases in sirt mRNA that were observed in the liver, muscle and lung tissues (Figure 2.8). These 4 members are known to have roles in upregulating antioxidants by activating FoxO1/3 transcription, upregulating antioxidant enzymes such as catalase, SOD2 and HO-1, and increasing the GSH/GSSH ratio (Kang et 
al., 2014; Liu et al., 2017; Olmos et al., 2013) (Table 2.12). These data suggest that Sirts have a role in antioxidant maintenance or upregulation in response to dehydration in $X$. laevis.

In the liver, mRNA levels of Sirt4, which is well characterized to suppress fatty acid oxidation (Laurent et al., 2013; Nasrin et al., 2010), were found to increase during dehydration (Table 2.1). However, mRNA levels of Sirt5, the member most responsible for deacetylating and activating CPS1 (Nakagawa et al., 2009b), did not increase (Figure 2.2). As it is already known that CPS1 activity significantly increases during $X$. laevis dehydration (Balinsky et al., 1961, 1967a), it can be postulated that, despite the decrease in sirt5 mRNA abundance, an increase in SIRT5 activity may be still be present given the increased global Sirt activity in the liver during dehydration (Figure 2.8). Alternatively, it is possible that Sirt5 has a smaller role in ureagenesis altogether in the context of $X$. laevis dehydration. As the mRNA of Sirts that are important for the regulation of metabolism, such as sirt4, are differentially regulated, the results suggest that Sirts play a role in the metabolic remodelling during whole-body dehydration in X. laevis. However, for the case of decreased sirt 5 mRNA which is the opposite of what is expected to promote ureagenesis, it is also evident that the "canonical" mechanisms depicted in other studies using cell lines and loss/gain-of-function methodologies may be not be occurring exactly the same way in dehydrated X. laevis where changes occur in a more complex, in vivo manner. As a result, while mechanistic studies that elucidated the roles of Sirts in cell lines provide valuable leads for the present studies in X. laevis, the differences expected between in vivo and in vitro must be acknowledged. In the case of sirt5 mRNA expression in the liver which unexpectedly decreased in response to dehydration, future 
studies aimed at assessing SIRT5 protein levels, SIRT5-specific deacetylase activity, and the acetylation status of CPS1 would provide further insight that addresses some of the limitations in the present thesis.

In addition to analyses that assess relative mRNA levels and global Sirt activity, relative abundance of Ac-SOD2 (K70) was assessed in order to determine whether the observed increases in Sirt mRNA and activity correlate with decreased acetylated proteins that are known target of Sirts. Indeed, the conserved K70 residue on $X$. laevis SOD2 protein (Figure 2.9) that is known to be deacetylated by Sirt3 specifically, is an action that has been previously shown to increase the antioxidant dismutase activity of SOD2 (Chen et al., 2011; Qiu et al., 2010). Western immunoblots from Chapter 2 showed that decreases in Ac-SOD2 (K70) appeared in the heart and kidney, with the strongest decreases in lung (Figures 2.10 and 2.11). As before, these results suggest that the regulation of cellular processes in vivo may not always be as straightforward as those seen in in vitro studies. For example, while the abundance of Ac-SOD2 in the kidney decreased in response to dehydration, there was no change in sirt3 mRNA (Figure 2.7) or global Sirt activity (Figure 2.8) in this tissue. It is possible that, despite no observed increase at the mRNA level, Sirt3 kidney protein is increasing during dehydration. Another possibility is that although there were no detectable changes in global Sirt activity in the kidney, Sirt3 protein, if isolated from other Sirts, would have demonstrated increased deacetylase activity. These points, however, are speculative and underscore the limitations of the present results and warrant that further studies be pursued. The same disconnect between sirt3 mRNA expression and Ac-SOD2 is observed in the heart and lung, as there were no changes in sirt3 mRNA (Figure 2.5), although global Sirt activity 
was found to increase in both tissues (Figure 2.8). Despite the limitations in the methodologies, the results in the present thesis suggest that Sirts have an active role in dehydrated $X$. laevis and yielded many new questions that can be addressed in further studies.

Specific Hypothesis 1 revisited: Even though Sirts do not appear to be responsive to whole-body dehydration in every tissue that was assessed, the results do suggest that Sirt signaling is important in whole-body dehydration, and may contribute to the upregulation of antioxidants in addition to the remodeling of metabolism in X. laevis.

\subsection{Sirt/PGC-1 $\alpha /$ FoxO mediated roles in antioxidant upregulation}

In Chapter 2, it was found that the mRNA encoding for Sirt proteins involved in antioxidant upregulation were increased during dehydration along with global SIRT activity in the liver, muscle, and lung, suggesting a potential antioxidant upregulation in these three tissues. A previous study has already shown that the FoxO-regulated antioxidant pathway was activated in the liver (but not the muscle) of dehydrated $X$. laevis, and was accompanied by increases in expression of SOD2 and catalase protein and mRNA (Malik and Storey, 2011). Sirt signaling appeared to be stronger in lung as compared to liver, as evidenced by larger increases to Sirt global activity in addition to steeper decreases Ac-SOD2 (K70). Yet, the FoxO pathway as a regulator of antioxidants, had yet to be investigated in this tissue. In Chapter 3, the role of Sirt proteins in antioxidant upregulation continue to be explored in lung, in addition to the Sirtupregulated PGC-1 $\alpha /$ FoxO antioxidant pathway, with results in lung compared to those in brain. 
In Chapter 2, no changes to sirt3 mRNA were found with whole-body dehydration in lung, despite increases in global Sirt activity and decreases in Ac-SOD2. This result was "non-canonical" when compared to the initial studies that documented the effects of sirt3 mRNA overexpression on the acetylation levels of SOD2. However, Chapter 3 revealed that in response to whole-body dehydration, total SIRT3 protein levels increased significantly in lung (Figure 3.1), an effect that was not observed in brain (Figure 3.2). This suggests that antioxidants are upregulated in X. laevis lung during dehydration by increasing Sirt activity through the upregulation of SIRT3 protein, but not sirt3 mRNA transcripts. As stated before, this underscores the fact that cellular responses in X. laevis whole-body dehydration are complex, and implicate other layers of regulation that are not investigated in this thesis, such as post-transcriptional regulation by noncoding RNAs.

The studies that provided the most significant foundation for Chapter 3 were those that elucidated the Sirt/PGC-1 $\alpha /$ FoxO signaling pathway in cell lines. The authors first demonstrated that artificial upregulation of SIRT1 increases the expression of antioxidant genes such as SOD2 and catalase, which further increase the antioxidant capacity of the cell (Olmos et al., 2013). However, when mRNA levels of pgcla and foxo3 were artificially knocked down (which also resulted in the suppression of the respective protein levels), SIRT1 overexpression could no longer induce the expression of antioxidant genes (Olmos et al., 2013). Secondly, the authors demonstrated that the overexpression of SIRT1 resulted in increases in the expression if pgcla and foxo3 (Olmos et al., 2013). Thirdly, the authors showed through chromatin immunoprecipitation assays that SIRT1 also directly binds to the promoter regions of 
pgcla and foxo3, in addition to the promoters of antioxidant genes including sod 2 and catalase (Olmos et al., 2013). Together, these findings indicated that Sirt1, PGC-1 $\alpha$, and FoxO3 each play crucial roles in the expression of antioxidant genes. As a result, these targets became the focus of Chapter 3 in which the aim was to demonstrate that the antioxidant response during whole-body dehydration of $X$. laevis involves the regulation of important targets at various levels in the cell.

The results were consistent with an activation of the Sirt1/PGC-1 $\alpha /$ FoxO3 pathway, as evidenced by increases in mRNA and protein levels. Similar to the responses in endothelial cells as previously described (Olmos et al., 2013), X. laevis upregulated PGC-1 $\alpha$ and FoxO3 mRNA and protein in lung, but not brain (Figures 3.3 and 3.6). The protein levels of SIRT1, however, were found to decrease in lung (Figure 3.1). This was unexpected given that sirt1 mRNA levels increased in lung in response to dehydration (Figure 2.6). Furthermore, it cannot be ruled out that, despite decreased total SIRT1 protein abundance, SIRT1 deacetylase activity may be increased in response to dehydration, which facilitates an increase in SIRT1 proteins associated to the promoter regions of pgcla, foxo3, and antioxidant genes. Indeed, the increase in global Sirt activity in the lungs of dehydrated $X$. laevis (Figure 2.8) supports this idea, but more studies are warranted for verification.

The increase in SIRT1 not only promotes antioxidant upregulation by direct interaction with promoter regions, but also through deacetylase activity. Previous experiments have also found that SIRT1 overexpression in cell lines promoted the formation of a PGC-1 $\alpha /$ FoxO3 protein complex with which antioxidants were upregulated (Olmos et al., 2013). Furthermore, when increased SIRT1 facilitated PGC- 
$1 \alpha /$ FoxO3 complex formation, both members exhibited decreased protein acetylation, which suggests that SIRT1 deacetylase activity is an important component of antioxidant regulation.

In addition to FoxO3, the related FoxO1 was also investigated for its role in the upregulation of antioxidants in X. laevis. Both FoxO1 and FoxO3 promote the transcription of antioxidant genes, and are regulated by inhibitory phosphorylation. As previously stated, the relative amounts of phosphorylated liver FoxO1 decrease in response to whole body dehydration in X. laevis (Malik and Storey, 2011). In the General Introduction, it has been stated that post-translational protein phosphorylation plays an important role in the dehydration response. Other studies have shown that protein acetylation plays as important of a role as protein phosphorylation in the regulation of cellular processes, as acetylation of FoxO1 is known to facilitate inhibitory phosphorylation. Indeed, SIRT1 deactylase activity is capable of upregulating antioxidants by deacetylating FoxO1, which results in decreased FoxO1 phosphorylation, and its subsequent localization to the nucleus (Awad et al., 2014; Daitoku et al., 2004; Matsuzaki et al., 2005). In Chapter 3, not only were FoxO1 and FoxO3 upregulated in lung on both mRNA and protein levels, but inhibitory phosphorylation on FoxO1 serine residues were also observed to decrease in response to dehydration (Figure 3.5B).

Together, both the results from the present thesis and the literature suggest that there is an upregulation in antioxidants which is mediated by the regulation of Sirt, PGC- $1 \alpha$, and FoxO1/3 transcription factors in X. laevis lung, but not brain.

Through the activation of Sirts, the results showed that the modifications are performed on the cellular level to facilitate the upregulation of antioxidants. This was 
further supported in Chapter 3, which showed an increase in protein level of catalase, and increases in both mRNA and protein levels of HO-1 in the lung (Figure 3.7). However, none of these changes were observed in the brain. Indeed, the expressions of these targets are both positively regulated by Sirts, PGC-1 $\alpha$, and FoxO1/3, which appeared to be upregulated in the lung. These transcriptional regulators are also known to promote the expression of SOD1 and SOD2, but with the exception of sod1 lung mRNA which increased, there were no changes in these antioxidant enzymes at either mRNA or protein levels in lung or brain (Figure 3.9). The results suggest that upregulation of antioxidants not only occur in a tissue-specific manner, but more specifically, catalase and HO-1 are regulated by SIRT1/PGC-1 $\alpha /$ FoxO1/3 in response to whole-body dehydration in $X$. laevis, whereas SOD2 activity is increased via SIRT3 activity (Figure 3.10).

With respect to antioxidant regulation, an interesting finding was that, out of the six tissues assessed, only the lung exhibited a significantly decreased antioxidant capacity in high dehydration conditions. Yet, results for lung also suggested a significantly stronger upregulation of antioxidants, as compared to other tissues like the brain. From one standpoint, this is a logical finding, as it is the select tissues that deplete antioxidants which require the highest antioxidant synthesis. It was hypothesized that the brain may upregulate antioxidants simply due to the importance of this organ. However, Sirts and regulators of antioxidants appeared to be relatively nonresponsive to dehydration. This result could lead to postulations that the increased blood circulation to the cranial region, as previously reported (Hillman and Sommerfeldt, 1981), may adequately compensate for 
any impairments in oxygen delivery to the brain that may be caused by decreased plasma volume.

Specific Hypothesis 2 revisited: The response to whole-body dehydration by $X$. laevis does appear to implicate the activation of Sirt/PGC-1 $\alpha /$ FoxO signaling pathway, as evidenced by antioxidant upregulation at the mRNA, protein, and protein posttranslational modification levels. However, antioxidant responses occur in a tissuespecific manner, and it is the specific tissues that exhibit decreased antioxidant capacity during dehydration that also demonstrate the strongest cellular signals that would mediate their upregulation.

\subsection{Sirt-mediated remodelling of metabolic genes via PGC-1 $\alpha$ and Hif-1a}

In Chapter 2, upregulations of sirt4 mRNA were observed which, according to the literature, is suggestive of an action to suppress fatty acid oxidation at the cellular level. As mentioned multiple times throughout this thesis, $X$. laevis dehydration has shown that whole-body dehydration in this amphibian causes a loss in plasma volume which impairs the circulation of oxygen throughout the body. It has been shown that $X$. laevis will continue to raise its resting heart rate in response to extreme dehydration, at which point the levels of whole-animal lactate are also observed to rise (Hillman, 1978). Given that (1) oxygen becomes a limiting factor for areas of the animal that rely on a normal circulatory system, and (2) the animal raises its whole-animal lactate levels at rest, it was hypothesized that $X$. laevis remodels its metabolism to suppress cellular processes in fatty acid oxidation while shifting more dependence on glycolysis to meet the energy demands of the cell. 
The nutrient sensor and transcriptional coactivator, PGC-1 $\alpha$, has been a major target of study in this thesis. In one review, a pair of PGC-1 $\alpha$ experts argued that this target is well known for promoting the mitochondrial biogenesis and it does so upregulating the genes required to produce proteins that facilitate the $\beta$-oxidation of fatty acids (Shoag and Arany, 2010) (Figure 4.1). At the same time, they pointed out interesting new data which demonstrated that PGC-1 $\alpha$ is actually upregulated in cells experiencing hypoxia (Shoag and Arany, 2010). They then proceeded to ask the obvious question: Why would a transcriptional activator that promotes the expression of genes related to oxygen-dependent metabolism be upregulated in low-oxygen conditions? This led them to hypothesize that PGC-1 $\alpha$ has different functions under hypoxia, which may differ from the traditional or "canonical" roles that has been well established in the literature and is based off of work done in normoxic conditions. With respect to wholebody dehydration in $X$. laevis, PGC-1 $\alpha$ is hypothesized to be upregulated based on the fact that it facilitates ureagenesis, which has been previously shown to occur (Balinsky et al., 1967b, 1961; Malik and Storey, 2009). As discussed in previous chapters, observed increases in Sirt activity is another reason to expect the upregulation of PGC-1 $\alpha$ as it is known to be activated by deacetylation. At the same time, a case can be made for its downregulation during $X$. laevis dehydration, which would prevent the expenditure of energy on the transcription of genes that are needed for oxygen-dependent metabolism.

In Chapter 4, PGC-1 $\alpha$ was characterized in the liver, skeletal muscle, and heart of $X$. laevis to investigate the cellular mechanisms by which Sirt-controlled transcription factors are regulating the expression of metabolic genes. Indeed, PGC-1 $\alpha$ was found to be upregulated in the liver of dehydrated $X$. laevis, whereas it was suppressed in the muscle 
and heart (Figure 4.3). Evidence for an active PGC-1 $\alpha$ response in the liver was further supported by increases in $m e f 2 c$ mRNA and increased nuclear localization of MEF2C protein (Figures 4.5 and 4.6), a transcription factor that is known to initiate pgcla gene expression. The same response for MEF2 was not observed in the muscle or heart, which correlates with the observed downregulation of PGC- $1 \alpha$ mRNA and protein in these two tissues (Figures 4.5 and 4.6). With respect to target downstream genes important for fatty acid oxidation, acyl-CoA dehydrogenase genes expectedly decreased with dehydration in muscle, but they also decreased in the liver (Figure 4.10). From these results, it was clear that the roles of PGC-1 $\alpha$ during dehydration were non-canonical, as its expression patterns were opposite to those of the acyl-CoA dehydrogenase genes in the liver.

In order to better understand the underlying mechanisms behind the suppression of liver acyl-CoA dehydrogenase genes during dehydration, the Hif-1 $\alpha$ transcription factor was also investigated in Chapter 4 (Figure 4.2). Hif-1 $\alpha$ was investigated not only because it is also activated by Sirts (Joo et al., 2015), but because it is known to suppress the expression of genes that promote fatty acid oxidation, including those that encode acyl-CoA dehydrogenases (Huang et al., 2014). Indeed, Hif-1 $\alpha$ protein and mRNA levels were shown to increase in multiple tissues of dehydrated $X$. laevis, including the liver, muscle, and heart (Figure 4.8). The Hif-1 $\alpha$ transcription factor is also well characterized to induce the expression of genes that are important for promoting glucose metabolism (Semenza, 2004), a process which was hypothesized to occur in dehydrated X. laevis when the circulation of oxygen becomes impaired. Indeed, increases in $h k, p d k 3$, and $p d k 4$ mRNA levels were observed in the liver of $X$. laevis under whole-body dehydration, which may play a role in the metabolic remodelling to anaerobic glycolysis (Figure 4.12). 
These results support the hypothesis that the regulation of genes at the cellular level is modulated during dehydration such that glucose metabolism if favoured over fatty acid oxidation.

As with the results from Chapters 2 and 3, the regulation of these pathways rarely proved to completely match the canonical responses that were previously established in the literature. In the skeletal muscle of dehydrated $X$. laevis, despite an increase in mRNA and protein of Hif-1 $\alpha$, the expression of several glycolytic genes ( $h k$ and $p f k$ ) were found to decrease, while mRNA levels of $p d k 3$ and $p d k 4$ increased (Figure 4.13). While these results show that the skeletal muscles may be inhibiting pyruvate dehydrogenase by inhibitory phosphorylation, they also suggest that both glucose and fatty acid metabolism are suppressed during dehydration. However, this result is consistent with a previous study that demonstrated that the skeletal muscles of $X$. laevis receive decreased blood circulation during dehydration, as opposed to the vital organs where circulation is maintained (Hillman and Sommerfeldt, 1981).

The results from Chapter 4 suggested that Sirt-controlled transcription factors, but mainly Hif- $1 \alpha$, were responsible for the remodelling of gene expression patterns to favour anaerobic glycolysis over oxygen-dependent metabolism. However, Chapter 4 provided results which suggested other roles for PGC- $1 \alpha$. Both PGC-1 $\alpha$ and Hif- $1 \alpha$ are known to upregulate vegf in independent pathways, and both transcription factors were observed to be upregulated in the liver. Consistently, vegf mRNA levels in the liver of dehydrated $X$. laevis increased with dehydration, suggesting a potential angiogenic response specific to the liver that may be occurring as a response to an impaired circulatory system. 
Lastly, an overlapping theme which was not thoroughly discussed in Chapter 4 was the role of PGC-1 $\alpha$ activation in the liver and its suppression in the skeletal muscles, but in the context of antioxidant regulation. In Chapter 3, it was discussed how PGC-1 $\alpha$ was one of three important targets for antioxidant upregulation (the other two being Sirt deacetylases and FoxO transcription factors). It was mentioned in the literature that compromising any one of these targets would have a negative effect on the antioxidant system (Olmos et al., 2013). Furthermore, the first study on FoxO1-mediated antioxidant control in the context of dehydrated $X$. laevis investigated the liver and muscle, and found a clear upregulation in FoxO1-regulated antioxidants in liver, but not muscle (Malik and Storey, 2011). One potential reason why antioxidants were not upregulated in the muscle of $X$. laevis may be due the observed decrease in PGC-1 $\alpha$ mRNA and protein during dehydration (Figure 4.3). This result would further suggest that $X$. laevis is prioritizing its vital organs over the skeletal muscles when encountering whole-body dehydration.

\section{Specific Hypothesis 3 revisited: The results from Chapter 4 found that the Sirt-} controlled PGC-1 $\alpha$ and Hif-1 $\alpha$ transcription factors were upregulated in response to $X$. laevis dehydration. Gene expression analyses did indicate that glucose metabolism was favoured over oxygen-dependent fatty acid oxidation particularly in the liver. Genes that encode functional proteins which contribute to angiogenesis and the ureagenesis were also observed to increase in the liver.

\subsection{Final conclusions}

Previous studies have characterized the physiological effects of whole-body dehydration in $X$. laevis. The results from these past studies have generated hypotheses 
with respect to the animal's responses at the cellular level, which were addressed in this thesis. In Chapter 2, a study of the 7 Sirt members suggested that (1) Sirts are regulating protein post-translational acetylation in response to whole-body dehydration, (2) Sirt activity may have downstream effects on antioxidant signaling and metabolism, and (3) known targets of Sirt action, such as Ac-SOD2, decrease in response to dehydration. In order to further understand Sirt-mediated antioxidant regulation in response to wholebody dehydration, the Sirt/PGC-1 $\alpha /$ FoxO pathway was studied in Chapter 3, which focused on lung and brain. The results revealed that antioxidant upregulation occurs in a tissue specific manner. Specifically, the tissues that appear to have antioxidant pools depleted during dehydration also exhibit the most significant antioxidant upregulation. Dehydration-induced restrictions in oxygen circulation were also shown to affect metabolic signaling pathways at the transcription factor and gene expression levels. Results from Chapter 4 showed that Sirt-mediated increases in PGC-1 $\alpha$ and Hif-1 $\alpha$, particularly in the liver, play broad roles to activate ureagenesis and angiogenesis, while suppressing fatty acid oxidation in favour of glucose metabolism. 


\subsection{References}

Awad, H., Nolette, N., Hinton, M., Dakshinamurti, S., 2014. AMPK and FoxO1 regulate catalase expression in hypoxic pulmonary arterial smooth muscle. Pediatr.

Pulmonol. 49, 885-897. doi:10.1002/ppul.22919

Balinsky, J.B., Choritz, E.L., Coe, C.G., van der Schans, G.S., 1967a. Amino acid metabolism and urea synthesis in naturally aestivating Xenopus laevis. Comp. Biochem. Physiol. 22, 59-68.

Balinsky, J.B., Choritz, E.L., Coe, C.G., van der Schans, G.S., 1967b. Amino acid metabolism and urea synthesis in naturally aestivating Xenopus laevis. Comp. Biochem. Physiol. 22, 59-68.

Balinsky, J.B., Cragg, M.M., Baldwin, E., 1961. The adaptation of amphibian waste nitrogen excretion to dehydration. Comp. Biochem. Physiol. 3, 236-244. doi:10.1016/0010-406X(61)90009-3

Chen, Y., Zhang, J., Lin, Y., Lei, Q., Guan, K.L., Zhao, S., Xiong, Y., 2011. Tumour suppressor SIRT3 deacetylates and activates manganese superoxide dismutase to scavenge ROS. EMBO Rep. 12, 534-541. doi:10.1038/embor.2011.65

Childers, C.L., Storey, K.B., 2016. Post-translational Regulation of Hexokinase Function and Protein Stability in the Aestivating Frog Xenopus laevis. Protein J. 35, 61-71. doi:10.1007/s10930-016-9647-0

Daitoku, H., Hatta, M., Matsuzaki, H., Aratani, S., Ohshima, T., Miyagishi, M., Nakajima, T., Fukamizu, A., 2004. Silent information regulator 2 potentiates Foxo1mediated transcription through its deacetylase activity. Proc. Natl. Acad. Sci. 101, 10042-10047. doi:10.1073/pnas.0400593101

Dawson, N.J., Biggar, Y., Malik, A.I., Storey, K.B., 2018. Increased transcript levels and kinetic function of pyruvate kinase during severe dehydration in aestivating African clawed frogs, Xenopus laevis. Comp. Biochem. Physiol. Part - B Biochem. Mol. Biol. doi:10.1016/j.cbpb.2018.01.003

Hillman, S.S., 1978. The roles of oxygen delivery and electrolyte levels in the dehydrational death of Xenopus laevis. J. Comp. Physiol. B 128, 169-175. doi:10.1007/BF00689481

Hillman, S.S., Sommerfeldt, R.W., 1981. Microsphere studies of amphibian systemic blood flow redistribution during dehydration, hypovolemia, and salt load. J. Exp. Zool. 218, 305-308. doi:10.1002/jez.1402180223

Huang, D., Li, T., Li, X., Zhang, L., Sun, L., He, X., Zhong, X., Jia, D., Song, L., Semenza, G.L., Gao, P., Zhang, H., 2014. HIF-1-mediated suppression of acyl-CoA dehydrogenases and fatty acid oxidation is critical for cancer progression. Cell Rep. 8, 1930-1942. doi:10.1016/j.celrep.2014.08.028 
Jacobs, K.M., Pennington, J.D., Bisht, K.S., Aykin-Burns, N., Kim, H.S., Mishra, M., Sun, L., Nguyen, P., Ahn, B.H., Leclerc, J., Deng, C.X., Spitz, D.R., Gius, D., 2008. SIRT3 interacts with the daf-16 homolog FOXO3a in the mitochondria, as well as increases FOXO3a dependent gene expression. Int. J. Biol. Sci. 4, 291-299. doi:10.7150/ijbs.4.291

Joo, H.Y., Yun, M., Jeong, J., Park, E.R., Shin, H.J., Woo, S.R., Jung, J.K., Kim, Y.M., Park, J.J., Kim, J., Lee, K.H., 2015. SIRT1 deacetylates and stabilizes hypoxiainducible factor-1 $\alpha$ (HIF-1 $\alpha)$ via direct interactions during hypoxia. Biochem. Biophys. Res. Commun. 462, 294-300. doi:10.1016/j.bbrc.2015.04.119

Kang, J., Jeong, M.G., Oh, S., Jang, E.J., Kim, H.K., Hwang, E.S., 2014. A FoxO1dependent, but NRF2-independent induction of heme oxygenase-1 during muscle atrophy. FEBS Lett. 588, 79-85. doi:10.1016/j.febslet.2013.11.009

Katzenback, B.A., Dawson, N.J., Storey, K.B., 2014. Purification and characterization of a urea sensitive lactate dehydrogenase from the liver of the African clawed frog, Xenopus laevis. J. Comp. Physiol. B Biochem. Syst. Environ. Physiol. 184, 601611. doi:10.1007/s00360-014-0824-1

Laurent, G., de Boer, V.C.J., Finley, L.W.S., Sweeney, M., Lu, H., Schug, T.T., Cen, Y., Jeong, S.M., Li, X., Sauve, A.A., Haigis, M.C., 2013. SIRT4 Represses Peroxisome Proliferator-Activated Receptor $\alpha$ Activity To Suppress Hepatic Fat Oxidation. Mol. Cell. Biol. 33, 4552-4561. doi:10.1128/MCB.00087-13

Liu, G., Park, S.-H., Imbesi, M., Nathan, W.J., Zou, X., Zhu, Y., Jiang, H., Parisiadou, L., Gius, D., 2017. Loss of NAD-Dependent Protein Deacetylase Sirtuin-2 Alters Mitochondrial Protein Acetylation and Dysregulates Mitophagy. Antioxid. Redox Signal. 26, 849-863. doi:10.1089/ars.2016.6662

Malik, A.I., Storey, K.B., 2011. Transcriptional regulation of antioxidant enzymes by FoxO1 under dehydration stress. Gene 485, 114-119.

doi:10.1016/j.gene.2011.06.014

Malik, A.I., Storey, K.B., 2009. Activation of extracellular signal-regulated kinases during dehydration in the African clawed frog, Xenopus laevis. J. Exp. Biol. 212, 2595-2603. doi:10.1242/jeb.030627

Matsuzaki, H., Daitoku, H., Hatta, M., Aoyama, H., Yoshimochi, K., Fukamizu, A., 2005. Acetylation of Foxo1 alters its DNA-binding ability and sensitivity to phosphorylation. Proc. Natl. Acad. Sci. 102, 11278-11283. doi:10.1073/pnas.0502738102

Nakagawa, T., Lomb, D.J., Haigis, M.C., Guarente, L., 2009a. SIRT5 Deacetylates Carbamoyl Phosphate Synthetase 1 and Regulates the Urea Cycle. Cell 137, 560570. doi:10.1016/j.cell.2009.02.026

Nakagawa, T., Lomb, D.J., Haigis, M.C., Guarente, L., 2009b. SIRT5 Deacetylates Carbamoyl Phosphate Synthetase 1 and Regulates the Urea Cycle. Cell 137, 560- 
570. doi:10.1016/j.cell.2009.02.026

Nasrin, N., Wu, X., Fortier, E., Feng, Y., Baré, O.C., Chen, S., Ren, X., Wu, Z., Streeper, R.S., Bordone, L., 2010. SIRT4 regulates fatty acid oxidation and mitochondrial gene expression in liver and muscle cells. J. Biol. Chem. 285, 31995-32002. doi:10.1074/jbc.M110.124164

Olmos, Y., Sánchez-Gómez, F.J., Wild, B., García-Quintans, N., Cabezudo, S., Lamas, S., Monsalve, M., 2013. SirT1 Regulation of Antioxidant Genes Is Dependent on the Formation of a FoxO3a/PGC-1 $\alpha$ Complex. Antioxid. Redox Signal. 19, 1507-1521. doi:10.1089/ars.2012.4713

Qiu, X., Brown, K., Hirschey, M.D., Verdin, E., Chen, D., 2010. Calorie restriction reduces oxidative stress by SIRT3-mediated SOD2 activation. Cell Metab. 12, 662667. doi:10.1016/j.cmet.2010.11.015

Rodgers, J.T., Lerin, C., Gerhart-Hines, Z., Puigserver, P., 2008. Metabolic adaptations through the PGC-1 $\alpha$ and SIRT1 pathways. FEBS Lett. doi:10.1016/j.febslet.2007.11.034

Semenza, G.L., 2004. Hydroxylation of HIF-1: Oxygen Sensing at the Molecular Level. Physiology 19, 176-182. doi:10.1152/physiol.00001.2004

Shoag, J., Arany, Z., 2010. Regulation of hypoxia-inducible genes by PGC-1 $\alpha$. Arterioscler. Thromb. Vasc. Biol. doi:10.1161/ATVBAHA.108.181636 


\section{Appendix A}

List of Publications 


\section{Published/Accepted/Submitted peer-reviewed journal articles}

20. Hawkins, L.J., Luu, B.E., Storey, K.B. Selection of reference genes for accurate RTqPCR analysis of dehydration tolerance in Xenopus laevis. J Comp Physiol B [Submitted and currently in review]

19. Biggar, K.K., Luu, B.E., Wu, C.-W., Pifferi, F., Perret, M., Storey, K.B. Identification of novel and conserved microRNA and their expression in the gray mouse lemur, Microcebus murinus, a primate capable of daily torpor. Gene. [Submitted and currently in revision].

18. Luu, B.E., Storey, K.B. (2018). Solving donor organ shortage with insights from freeze tolerance in nature. Bioessays. Accepted.

17. Holahan, M.R., Smith, C.A., Luu, B.E., Storey, K.B. (2018). Preadolescent phthalate (DEHP) exposure is associated with elevated locomotor activity and reward-related behavior and a reduced number of tyrosine hydroxylase positive neurons in postadolescent male and female rats. Toxicol Sci. doi: 10.1093/toxsci/kfy171. [Epub ahead of print]

16. Zhang, Y., Luu, B.E., Storey, K.B. (2018). FoxO4 activity is regulated by phosphorylation and the cellular environment during dehydration in the African clawed frog, Xenopus laevis. Biochim Biophys Acta. 1862(8):1721-1728. doi: 10.1016/j.bbagen.2018.05.002.

15. Seibel, B.A., Luu, B.E., Tessier, S.N., Towanda, T., Storey, K.B. (2017). Metabolic suppression in the pelagic crab, Pleuroncodes planipes, in oxygen minimum zones. Comp Biochem Physiol B Biochem Mol Biol. pii: S1096-4959(17)30213-0. doi: 10.1016/j.cbpb.2017.12.017. [Epub ahead of print]

14. Luu, B.E., Wijenayake, S., Malik A.I., Storey, K.B. (2018). The regulation of heat shock proteins in response to dehydration in Xenopus laevis. Cell Stress Chaperones. 23(1): 45-53. doi: 10.1007/s12192-017-0822-9. Epub 2017 Jul 5.

13. Luu, B.E., Wijenayake, S., Zhang, J., Tessier, S.N., Quintero-Galvis, J.F., GaitánEspitia, J.D., Nespolo, R.F., Storey, K.B. (2017). Strategies of biochemical adaptation for hibernation in a South American marsupial, Dromiciops gliroides: 2. Control of the Akt pathway and protein translation machinery. Comp Biochem Physiol B Biochem Mol Biol. doi: 10.1016/j.cbpb.2017.12.006. [Epub Dec 2017]

12. Luu, B.E., Wijenayake, S., Zhang, J., Tessier, S.N., Quintero-Galvis, J.F., GaitánEspitia, J.D., Nespolo, R.F., Storey, K.B. (2017). Strategies of biochemical adaptation for hibernation in a South American marsupial, Dromiciops gliroides: 3. Activation of pro-survival response pathways. Comp Biochem Physiol B Biochem Mol Biol. doi: 10.1016/j.cbpb.2017.12.005. [Epub Dec 2017] 
11. Wijenayake, S., Luu, B.E., Zhang, J., Tessier, S.N., Quintero-Galvis, J.F., GaitánEspitia, J.D., Nespolo, R.F., Storey, K.B. (2017). Strategies of biochemical adaptation for hibernation in a South American marsupial Dromiciops gliroides: 1. Mitogen-activated protein kinases and the cell stress response. Comp Biochem Physiol B Biochem Mol Biol. doi: 10.1016/j.cbpb.2017.12.007. [Epub Dec 2017]

10. Wijenayake, S., Luu, B.E., Zhang, J., Tessier, S.N., Quintero-Galvis, J.F., GaitánEspitia, J.D., Nespolo, R.F., Storey, K.B. (2017). Strategies of biochemical adaptation for hibernation in a South American marsupial, Dromiciops gliroides: 4. Regulation of pyruvate dehydrogenase complex and metabolic fuel selection. Comp Biochem Physiol B Biochem Mol Biol. doi: 10.1016/j.cbpb.2017.12.008. [Epub Dec 2017]

9. Tessier, S.N., Luu, B.E., Smith, J.C., Storey, K.B. (2017). The role of DNA methylation and histone post-translational modifications during mammalian hibernation. Cryobiology. doi: 10.1016/j.cryobiol.2017.02.008

8. Luu, B.E., Green, S.R. Childers, C.C., Holahan, M.R., Storey K.B. (2017). The roles of hippocampal microRNAs in response to acute postnatal exposure to $\operatorname{di}(2-$ ethylhexyl) phthalate in female and male rats. Neurotoxicology. doi: 10.1016/j.neuro.2017.02.004

7. Luu, B.E., Biggar, K.K., Wu, C.W., Szereszewski, K.E., and Storey, K.B. (2016). Torpor-responsive expression of species-specific microRNA in the thirteen-lined ground squirrel, Ictidomys tridecemlineatus. FEBS Letters. doi: 10.1002/18733468.12435

6. Hadj-Moussa, H., Moggridge, J.A., Luu, B.E., Quintero-Galvis, J.F., Gaitan-Espitia, J.D., Nespolo, R.F., Storey, K.B. (2016). The hibernating South American marsupial, Dromiciops gliroides, displays torpor-sensitive microRNA expression patterns. Scientific Reports. doi: 10.1038/srep24627

5. Wu, C.W., Biggar, K.K., Luu, B.E., and Storey, K.B. (2016). Analysis of microRNA expression during the torpor-arousal cycle of a mammalian hibernator, the 13-lined ground squirrel. Physiological Genomics._doi: 0.1152/physiolgenomics.00005.2016

4. Logan, S.M., Luu, B.E., and Storey, K.B. (2016). Turn down genes for WAT? Activation of anti-apoptotic pathways protects white adipose tissue in metabolically depressed thirteen-lined ground squirrels. Mol Cell Biochem. doi: 10.1007/s11010016-2695-0

3. Bansal, S., Luu, B.E., and Storey, K.B. (2016) MicroRNA regulation in heart and skeletal muscle over the freeze-thaw cycle in the freeze tolerant wood frog. J Comp Physiol B. 186(2): 229-241. doi: 10.1007/s00360-015-0951-3. 
2. Luu, B.E. and Storey, K.B. (2015) Dehydration triggers differential microRNA expression in Xenopus laevis brain. Gene, 573(1): 64-9. doi:

10.1016/j.gene.2015.07.027

1. Luu, B.E., Tessier, S.N., Duford, D., and Storey, K.B. (2015). The regulation of troponins I, C and ANP by GATA4 and Nkx2-5 in heart of hibernating thirteenlined ground squirrels, Ictidomys tridecemlineatus. PLoS One. 10(2):e0117747. doi: 10.1371/journal.pone.0117747.

\section{Completed projects (not submitted)}

Luu, B.E., Storey, K.B. Elucidating the molecular mechanisms of whole-body vertebrate dehydration: Lessons from an established vertebrate amphibian animal model, Xenopus laevis. (Review article)

Luu, B.E., Watts, A., Holahan, M.R., Storey, K.B. Regulation of the CREB transcription factor and its downstream targets in the hippocampus of female and male rats following acute postnatal exposure to di(2-ethylhexyl) phthalate.

Luu, B.E., Al-Attar, R., Zhang, J. Storey, K.B. Differential expression of genes from the mitochondrial genome of the anoxia-tolerant gray tree frog.

Zhang, J.*, Gerber, A.E.M.*, Luu, B.E., Storey, K.B. Expression and characterization of microRNAs in the cortex and brainstem of hibernating thirteen-lined ground squirrels, Ictidomys tridecemlineatus.

Luu, B.E., Biggar, K.K., Storey, K.B. Identification and characterization of novel microRNAs in the freeze-tolerant hatchling turtle, Chrysemys picta.

Luu, B.E., Wu, C.W., Storey, K.B. The African clawed frog naturally upregulates antioxidants in the kidney to combat dehydration-induced ischemia/reperfusion stress in the kidney.

Luu, B.E., Wu, C.W., Storey, K.B. Pyruvate dehydrogenase is sensitive to dehydrationinduced ischemia/reperfusion stress in Xenopus laevis.

Luu, B.E., Zhang, Y., Storey, K.B. Downstream targets of FoxO and Akt signaling contributes to muscle maintenance in the dehydrating African clawed frog, Xenopus laevis.

Luu, B.E., Storey, K.B. Differential regulation of microRNAs in the skeletal muscle of hibernating brown bears, Ursus arctos.

Luu, B.E., Smolinski, M.B., Waddington-Lamont, E., Abizaid, A., Storey, K.B. Brain region and temporal variables are dependent variables in the differential expression of brain microRNAs induced by social defeat stress. 


\section{Appendix B}

Scientific Communications at Meetings 


\section{$\underline{\text { Research proposal competitions }}$}

Luu, B.E., Chandravanshi, B., Hanini, A., Kueckelhaus, M. (2018). Transient activation of endogenous antioxidant pathways in donor livers prior to organ procurement by non-coding RNAs. Summit for Organ Preservation, Harvard Medical School

*This research proposal reached the semi-final stage. The proposal was also published in Cryobiology by the conference organizers, and its core ideas were included in a recently accepted article in Bioessays by Luu, B.E. \& Storey, K.B.

\section{Oral presentations (* - presenting author)}

Luu, B.E.,* Biggar, K.K., Wu, C.W., Storey, K.B. (2016) Hibr-miRs: Cold-sensitive novel microRNA in the hibernating 13-lined ground squirrel. CRYO 2016: The $53^{\text {rd }}$ Annual Meeting of the Society for Cryobiology. July 27, 2016.

Luu, B.E.* and Storey, K.B. (2015) Identification and characterization of speciesspecific novel microRNAs in the hibernating 13-lined ground squirrel, Ictidomys tridecemlineatus. 18th Chemistry \& Biochemistry Graduate Research Conference, Concordia Univ., Nov. 20, 2015

Luu, B.E.* and Storey, K.B. (2015) The hibernating thirteen-lined ground squirrel heart: A new model for heart research. University of Ottawa Heart Institute Research Day Coralie Lalonde Innovation Award Semi-finalist.

Luu, B.E.* and Storey, K.B. (2015) Dehydration triggers differential microRNA expression in Xenopus laevis brain. $12^{\text {th }}$ Annual Ottawa-Carleton Institute of Biology Symposium. Regional conference.

Luu, B.E.* and Busslinger, M. (2011) Transcriptional control of Pax5 by Ebf1 in early B-cell development. Vienna Biocenter Summer Student Symposium. Institutional conference. (Summer internship work)

\section{$\underline{\text { Invited Lectures }}$}

The study of non-coding RNAs in non-model organisms (Fall 2017). Graduate course (BIO 8361) at the University of Ottawa.

Introduction to the Cell Cycle (Fall 2015). $3^{\text {rd }}$ year undergraduate Cell Biology (BIOL 3201) at Carleton University.

Quantification of microRNAs (Fall 2014). Graduate course (BIO 8361) at the University of Ottawa. 


\section{Conference presentations}

Seibel, B., Luu, B.E., Tessier, S.N., Storey, K.B. Critical oxygen and metabolic suppression in the pelagic red crab, Pleuroncodes planipes. Canadian Society of Zoologists, University of Manitoba, Winnipeg, MB, May 15-19, 2017.

Luu, B.E., Holahan, M.R., and Storey, K.B. Hippocampal microRNA expression and dendrite development is disrupted by di(2-ethylhexyl) phthalate exposure in male, but not female rats. Ontario Life Science Day, Ottawa, ON, May 5, 2017.

Luu, B.E., Biggar, K.K., Wu, C.W., and Storey, K.B. Torpor-responsive expression of species-specific microRNA in the thirteen-lined ground squirrel, Ictidomys tridecemlineatus. Ottawa-Carleton Institute for Biology Conference, 2016.

Hadj-Moussa, H., Moggridge, J.A., Luu, B.E., Quintero-Galvis, J.F., Gaitán-Espitia, J., Nespolo, R.F., and Storey, K.B. Torpor triggers differential microRNA expression in hibernating South American marsupials, Dromiciops gliroides. 18th Chemistry \& Biochemistry Graduate Research Conference, Concordia Univ., Nov. 20, 2015

Smolinski, M.B.., Luu, B.E., Waddington-Lamont, E., Storey, K.B. Abizaid, A. Identification of stress-responsive brain microRNAs in mice by quantitative PCR. $7^{\text {th }}$ Annual Young Researcher's Conference, Royal Ottawa Hospital. May 7, 2015.

Luu, B.E. and Storey, K.B. The regulation of troponins I, C and ANP by GATA4 and Nkx2-5 in heart of hibernating 13-lined ground squirrels Ictidomys tridecemlineatus. University of Ottawa Heart Institute Research Day, University of Ottawa, May 5, 2015

Tessier, S.N., Luu, B.E. and Storey, K.B. Adaptations of cardiac muscle function during mammalian hibernation. Organ Banking Summit, Palo Alto, CA, Feb. 26-28, 2015.

Wijenayake, S., Luu, B.E. and Storey, K.B. Heat shock protein response during dehydration in an African clawed frog (Xenopus laevis). 17th Chemistry \& Biochemistry Graduate Research Conference, Concordia Univ., Nov. 28, 2014.

Tessier, S.N., Luu, B.E. and Storey, K.B. Adaptations of cardiac muscle function during mammalian hibernation. Society for Experimental Biology, Manchester University, Manchester, UK, July 1-4, 2014.

Tessier, S.N., Luu, B.E. and Storey, K.B. Adaptations of cardiac muscle function during mammalian hibernation. 3rd International Space Health Forum on Human Energy Conservation on Earth and in Space. Old Dominion University, Hampton, Virginia, April 3-4, 2014.

Tessier, S.N., Luu, B.E. and Storey, K.B. Adaptations of cardiac muscle function during mammalian hibernation. ACCryo2014, Key Largo, Florida, January 15-19. (2014). 
Tessier, S.N., Luu, B.E. and Storey, K.B. Adaptations of cardiac muscle function during mammalian hibernation. CRYO2013, 50th Annual Meeting, Society for Cryobiology, Bethesda, Maryland, July 28-31 (2013).

Luu, B.E. and Storey, K.B. Akt extinguishes energy expensive activities in the estivating African clawed frog. $1^{\text {st } I n t e r n a t i o n a l ~ C o n f e r e n c e ~ o n ~ O x i d a t i v e ~ S t r e s s ~ i n ~ A q u a t i c ~}$ Ecosystems. Los Cabos, Mexico (2012).

Luu, B.E. and Storey, K.B. Akt extinguishes energy expensive activities in the estivating African clawed frog. Canada-China Systems Biology Conference (2012).

Luu, B.E. and Storey, K.B. Role of myogenic regulatory factors in resistance to muscle disuse atrophy in anoxia tolerant turtles. Concordia University's 15 th Chemistry and Biochemistry Conference (2011).

Luu, B.E. and Storey, K.B. Role of myogenic regulatory factors in resistance to muscle disuse atrophy in anoxia tolerant turtles. Canadian Society of Zoologists' Annual National Conference (2011).

Tessier, S.N., Luu, B.E. and Storey, K.B. Adaptations of cardiac muscle function during mammalian hibernation. Canadian Society of Zoologists, Annual National Conference (2011).

Luu, B.E., Tessier, S.N., and Storey, K.B. The role of the troponin-tropomyosin complex and dystrophin-related glycoproteins in the hibernating thirteen-lined ground squirrel.26th Annual meeting, Federação de Sociedades de Biologia Experimental (FeSBE), Rio de Janeiro, Brazil, (2011).

Luu, B.E. and Storey, K.B. Resistance to skeletal muscle disuse atrophy in the hibernating 13-lined ground squirrel. Ottawa-Carleton Institute for Biology Conference, (2010). 


\section{Appendix C}

\section{Primer design for RT-qPCR}


This thesis involved large amounts of RT-qPCR. It is important that proper verifications were conducted in order to insure that accurate relative gene quantification was properly performed. All genes that were assessed in this thesis are annotated on Xenopus laevis genome databases available to the public. All quantified genes are located at http://www.xenbase.org and/or http://www.ncbi.nlm.nih.gov/gene

As previously explained (Session et al., 2016), the X. laevis allotetraploid genome has two sets of chromosomes (the L and S sets). Their results showed that for many processes, such as development, both sets of chromosomes contribute to gene expression. In other cases, only one set was used for gene expression, whereas the other was considered to be "silenced".

This thesis used published transcriptomic data of adult $X$. laevis tissues to identify the whether the mRNA sequenced associated with the L or S chromosome sets should be used for primer design. In all cases, the chromosome sets that showed higher gene expression was used for primer design in this thesis.

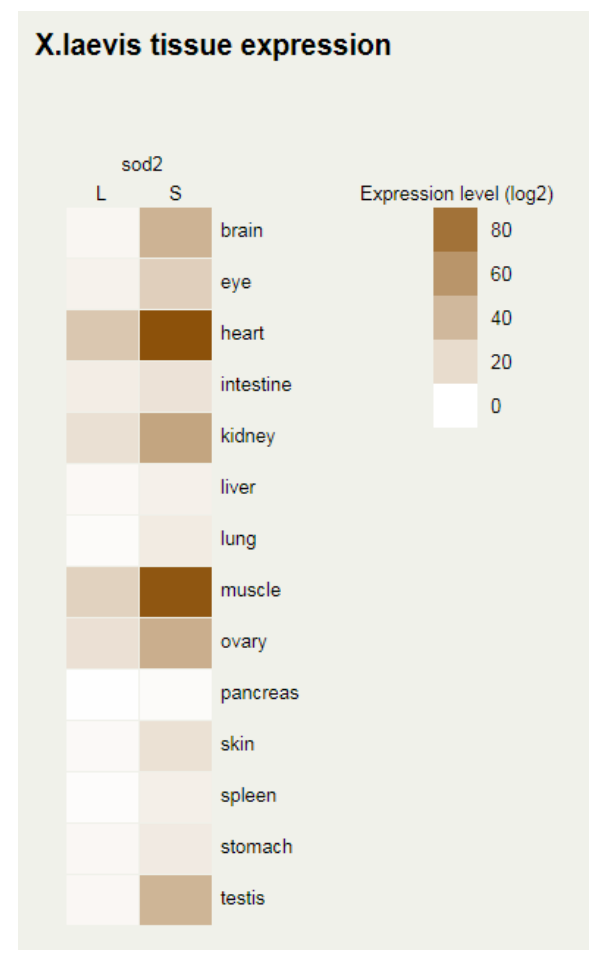

By choosing to study the gene variant from the chromosome set that undergoes higher gene expression, the assumption is made that the "silenced" chromosome, see (Session et al., 2016), also plays no role during dehydration. It is acknowledged in this thesis that these "silenced" chromosome sets may exhibit differential gene expression in response to dehydration. While this is a valid question that warrants further investigation, it is outside the scope of the present thesis. 
In this example, once it was decided to perform relative gene expression on sod2.s rather than sod2.l (sod2.s is the variant that showed higher expression), the sequence for the gene was obtained from Xenbase or NCBI as shown:

\begin{tabular}{|c|c|c|c|}
\hline $\begin{array}{l}\text { Xenbase } \\
\text { Gene ID }\end{array}$ & XB-GENE-1000989 & XB-GENE-1000995 & XB-GENE-17334272 \\
\hline Gene Symbol & sod2 & sod2.L & sod2.S \\
\hline Chromosome & Chr05 & chr5L & chros \\
\hline Molecules & tropicalis & laevis.L & laevis.S \\
\hline Gene & 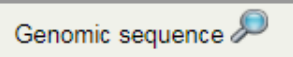 & Genomic sequence $\curvearrowright$ & Genomic sequence $\odot$ \\
\hline \multirow[t]{2}{*}{ CDS } & Gene model $\varnothing$ & & \\
\hline & Entrez Gene & Entrez Gene & Entrez Gene \\
\hline mRNA & $\underline{\text { Refseg }} \Rightarrow \varnothing$ & $\underline{\text { Refseg }} \nRightarrow \mathscr{P}$ & $\underline{\text { Refseg }} \Rightarrow P$ \\
\hline Protein $\mathbf{B}$ & $\underline{\text { Refseg }} \Rightarrow \rho_{\text {TrEMBL }}$ & $\underline{\text { Refseg }} \Rightarrow \rho_{\text {TrEMBL }}$ & Refseg $z^{\prime} \&_{\text {TrEMBL }}$ \\
\hline
\end{tabular}

This leads to the mRNA sequence of sod2.s, as shown on NCBI:

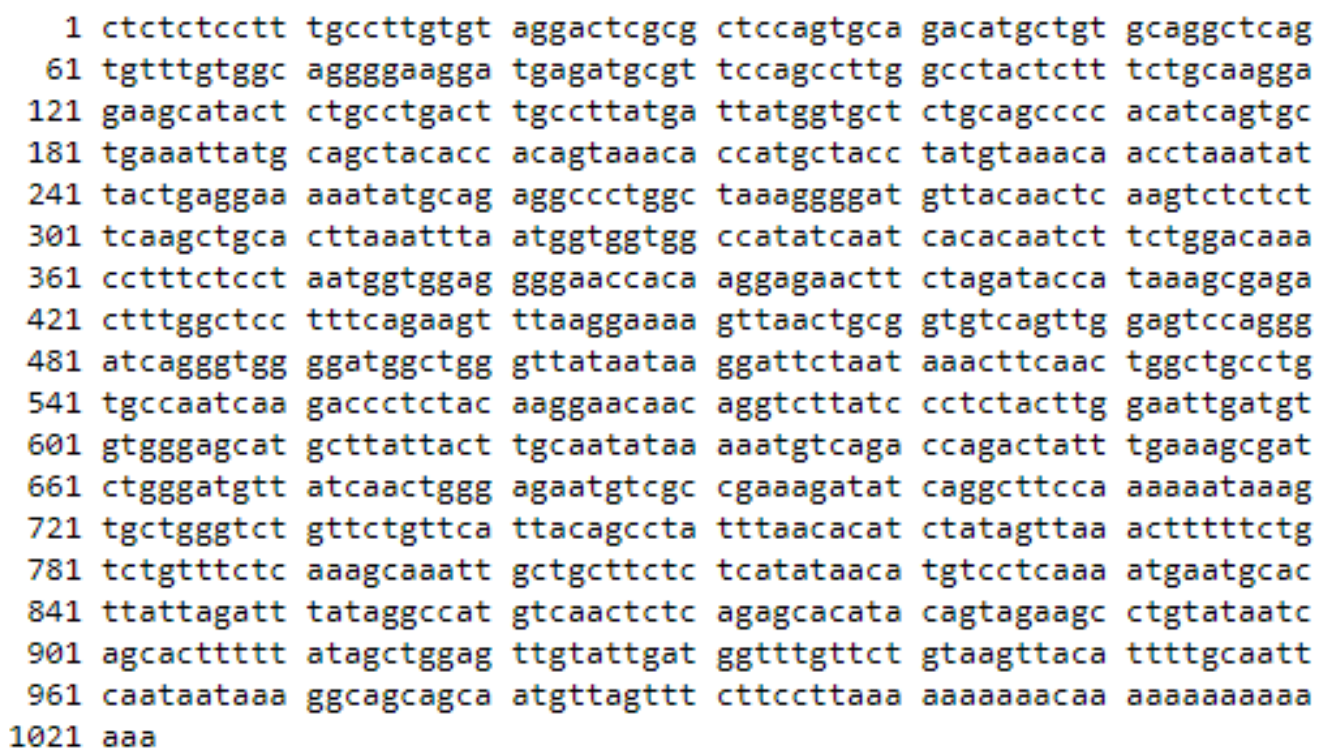


To design primers based on this sequence, NCBI Primer Blast was used to design primers that would amplify PCR products of 70-200 bp:

\section{NIH U.S. National Library of Medicine}

\section{Primer-BLAST}

\section{NCBI National Center for Biotechnology Information}

A tool for finding specific primers

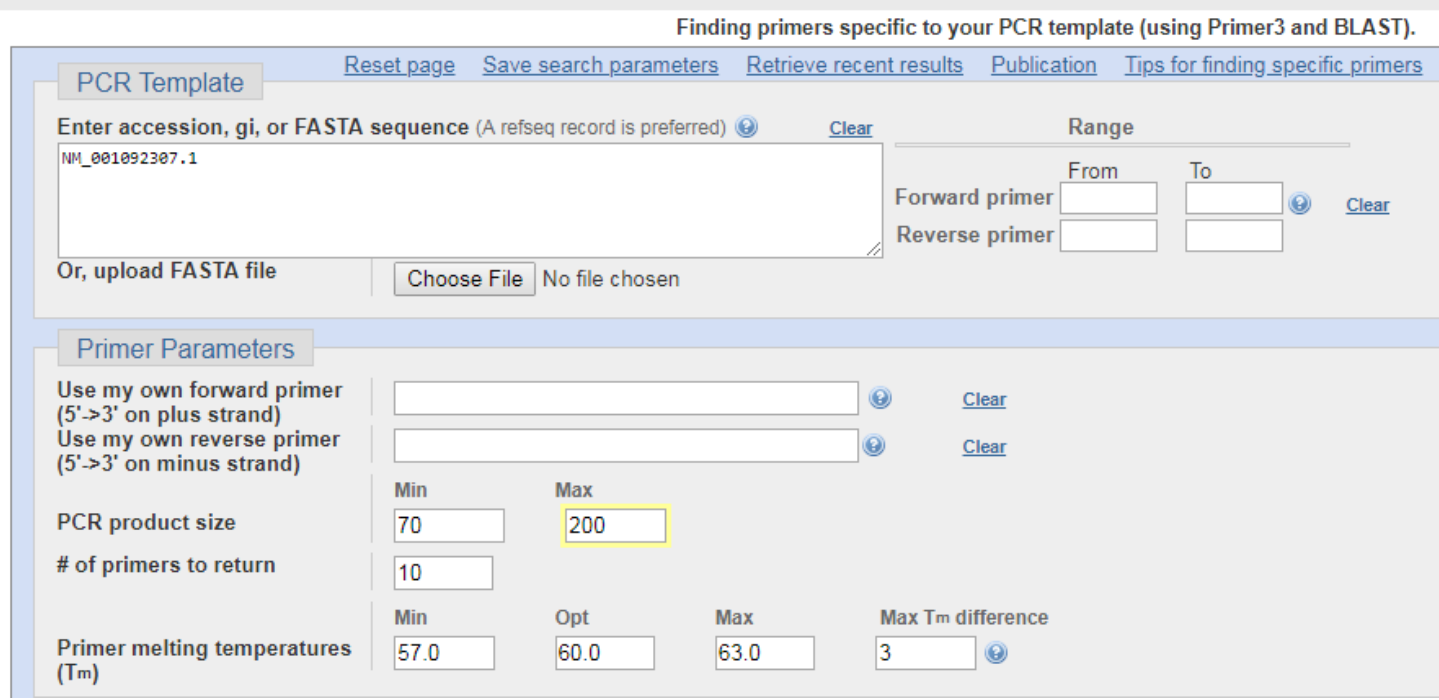

Importantly, Primer-BLAST is set to design primers that will amplify the input sod2.s mRNA sequence specifically, and not any other known $X$. laevis mRNA sequence.

Furthermore, an option is used to only design primer pairs that are separated by an intron on genomic DNA. This prevents the amplification of trace albeit present amounts of genomic DNA in the sample:

Intron inclusion

Primer pair must be separated by at least one intron on the corresponding genomic DNA (?)

NCBI Primer-BLAST uses the Primer3 algorithm to suggest that are expected to exhibit minimal primer homodimerization and heterodimerization.

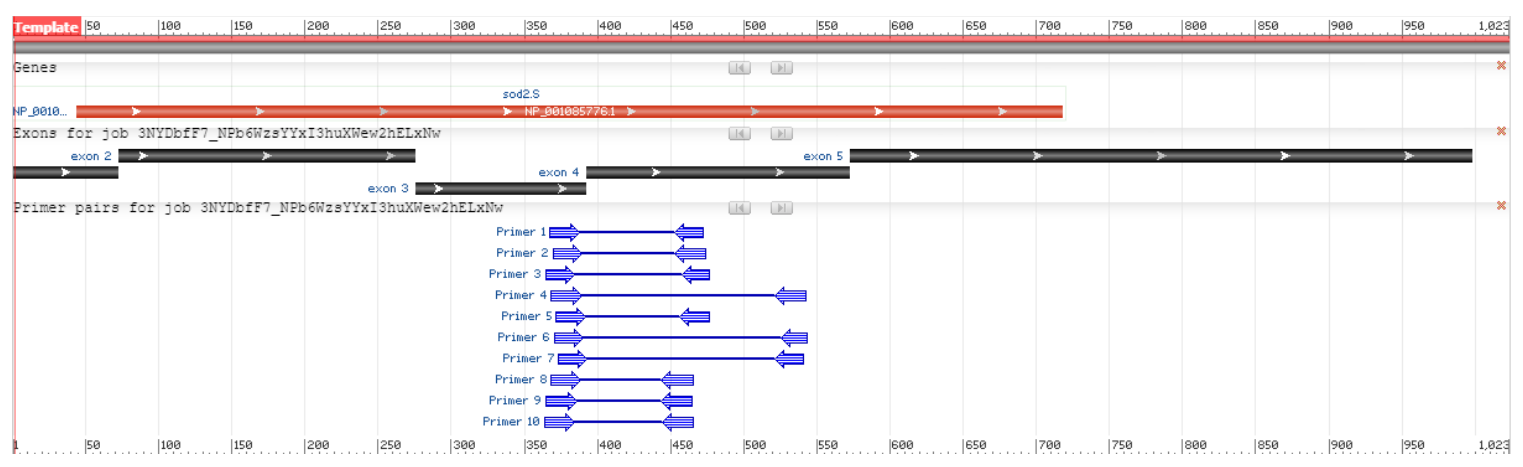




\section{Appendix D}

Assessment of RNA Integrity 


\section{A}

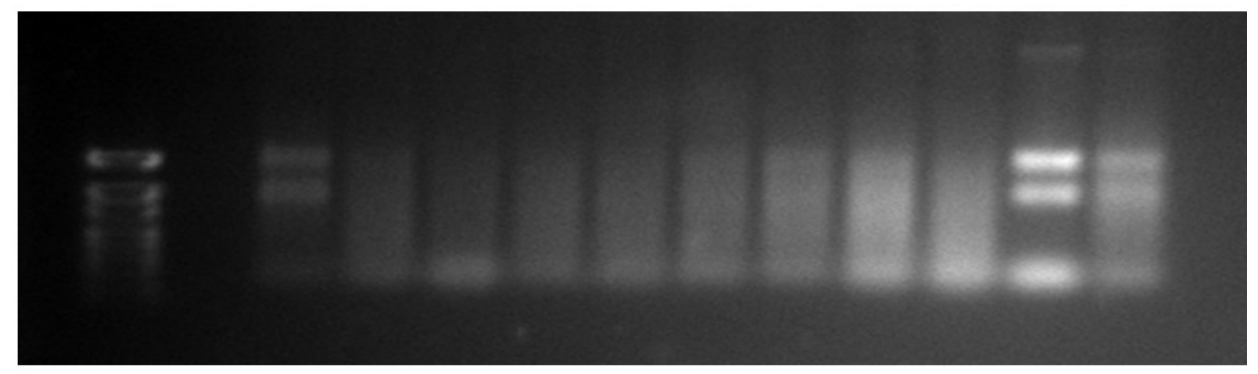

B

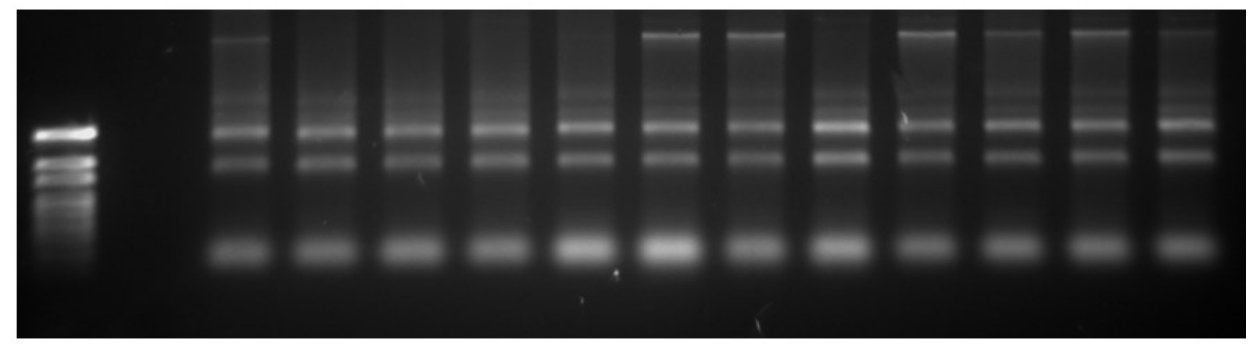

Control Medium High

C

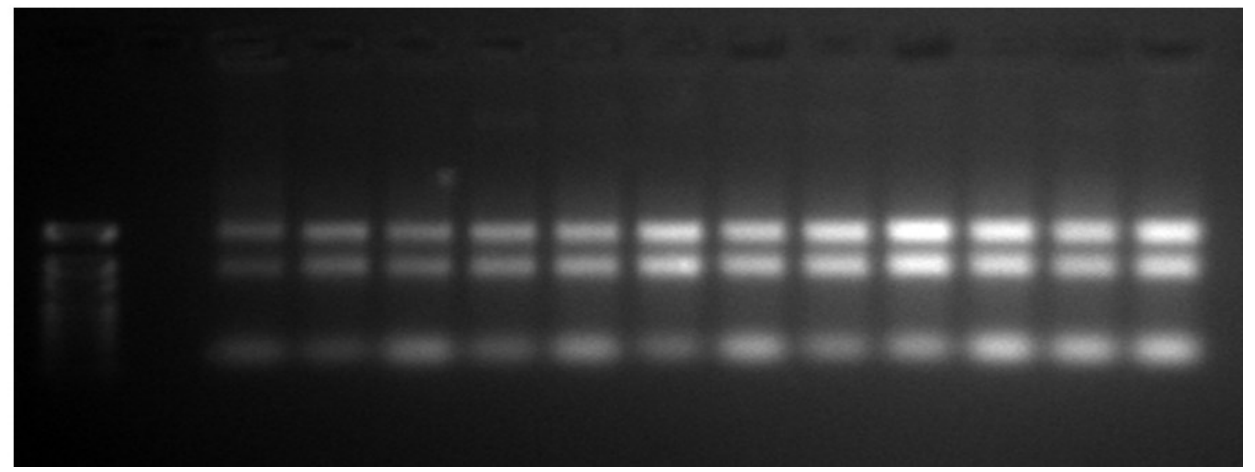

Figure D1. Following RNA extraction, RNA samples were run on an agarose gel to assess their integrity. RNA samples that produced smears (A) were deemed degraded and were not used. Other samples that contain significant amounts of DNA contamination (B) yielded a high molecular weight band and were treated with an additional round of RNA extraction to remove contaminating DNA. Samples that were used for cDNA synthesis (C) showed no signs of degradation or DNA contamination. 


\section{Appendix E}

\section{Controls for Gene Quantification using RT-qPCR}




\section{Use of Standard Curves}

Primers that successfully amplify one PCR product must further be assessed to determine the rate at which they amplify their target. For example, a properly functional primer set should double the amount of PCR product each cycle. However, since some primer sets do not bind perfectly to their targets, more than one PCR cycle may be required for a doubling of PCR product. Proper gene quantification requires that these differences in rates of amplification, also known as "primer efficiency" be taken into account.

In order to test a particular primer set to assess how efficiently it is amplifying a particular target, a standard curve must be performed. Standard curves are best done using a pooled sample of cDNA from both control and stress samples. Serial dilutions of pooled samples are then done and used to run a standard curve, as follows:

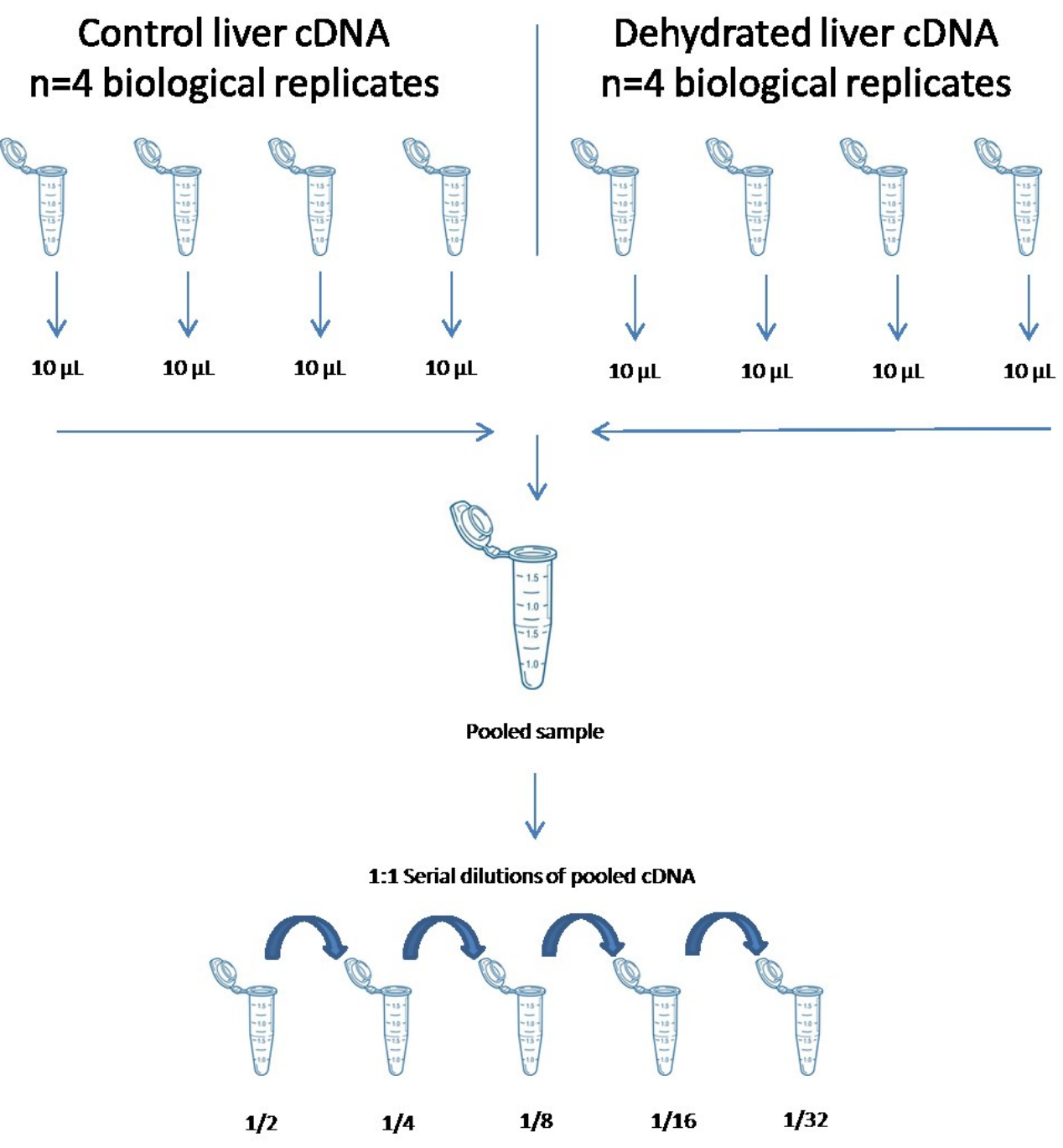


A standard curve is generated using the RT-qPCR protocol and it can be observed that not all primers will exactly double the amount of PCR product every cycle, and as a result, the primer will produce a \% Efficiency (E) that is less than $100 \%$. For every gene quantified in this thesis, the \% efficiency was accounted for when performing relative gene expression. In the following figure, circles represent standards of known dilutions $(1 / 2,1 / 4,1 / 8,1 / 16$, etc. $)$ and " $x$ " are individual biological replicate samples.

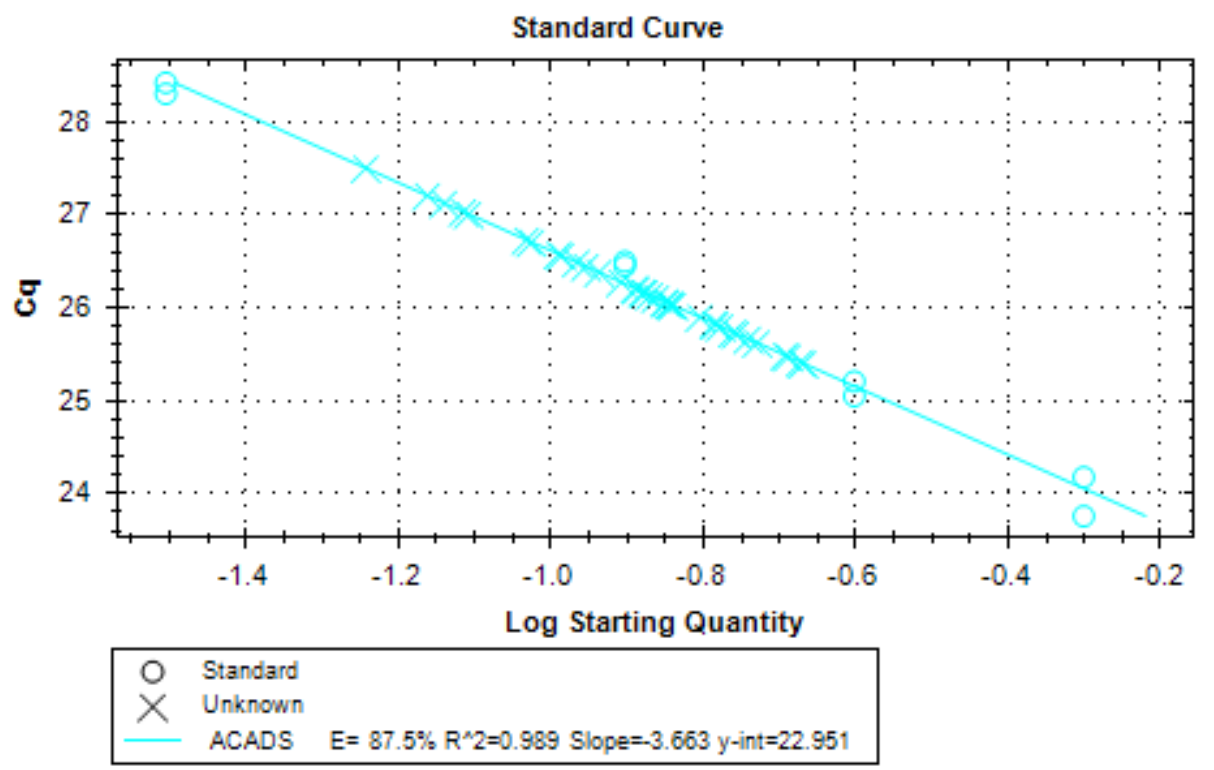

Despite not having $100 \%$ efficiency, the primer performs as it should in other aspects. For example, it is properly amplifying only one PCR product, as evidenced by the presence of one peak the melt curve analysis, which is performed for the quantification of all genes.

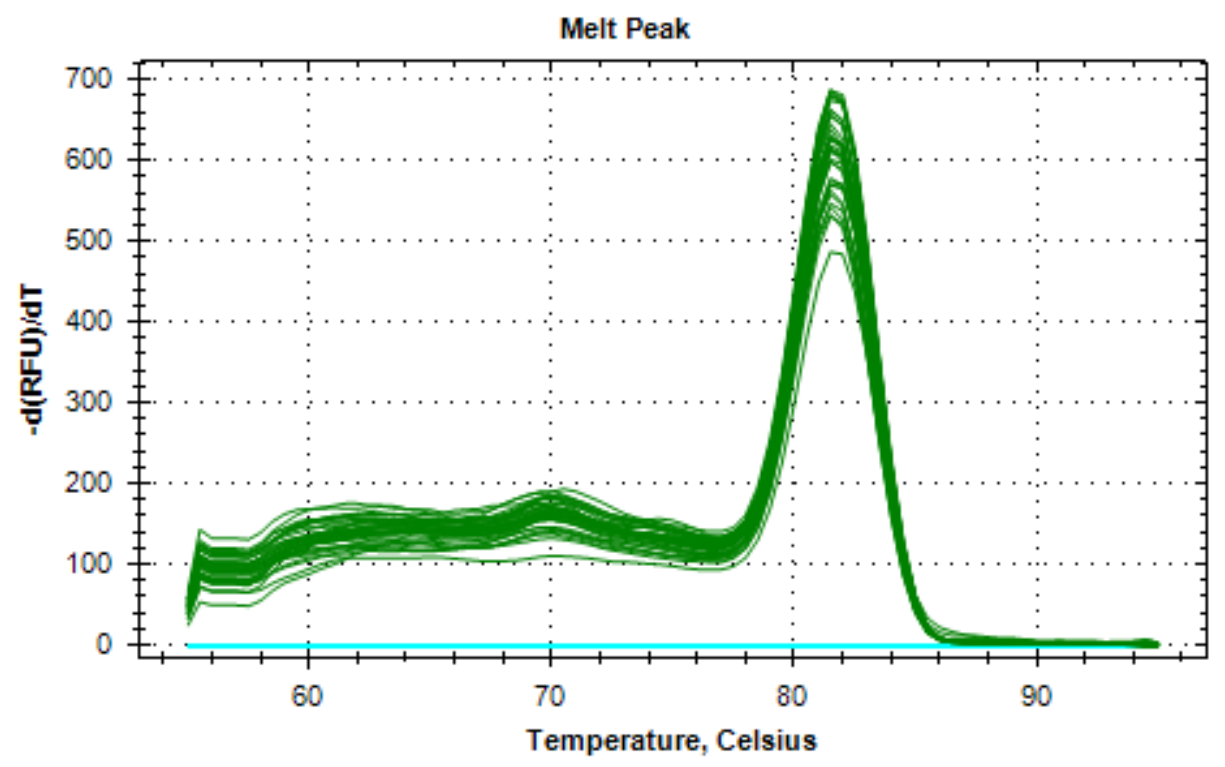


As an example, the following primer for $p g c l b$ was $\underline{\text { NOT }}$ suitable for gene quantification and required redesigning/optimization:
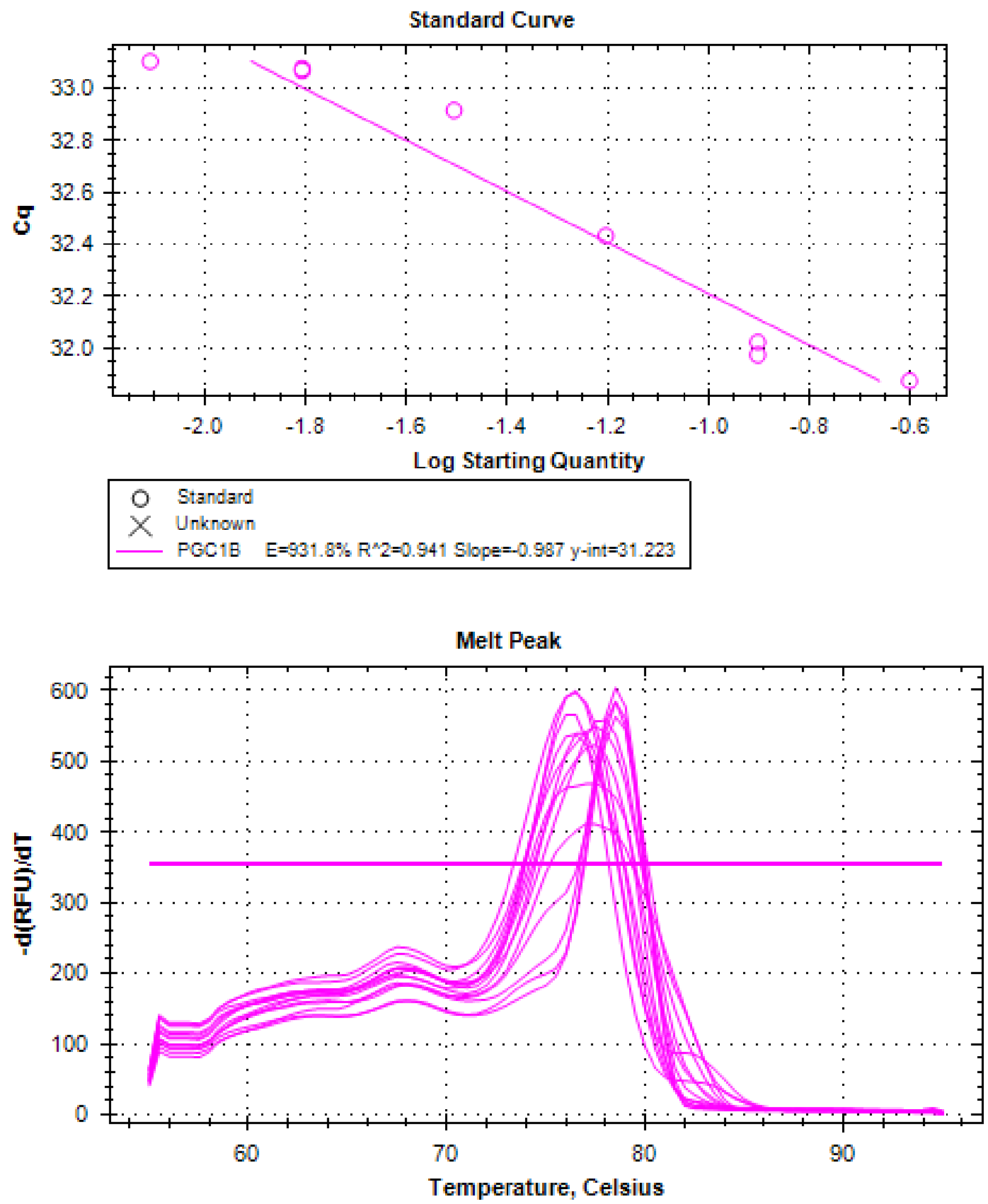

This primer set for $p g c 1 b$ did not produce a proper standard curve, and its respective melt curve analysis suggested that its reaction efficiency may be unusually high due to amplification of multiple nonspecific products other than the intended target. 
Indeed, another primer pair was designed for $p g c 1 b$ and using the same reaction and cDNA samples, a better reaction was achieved:
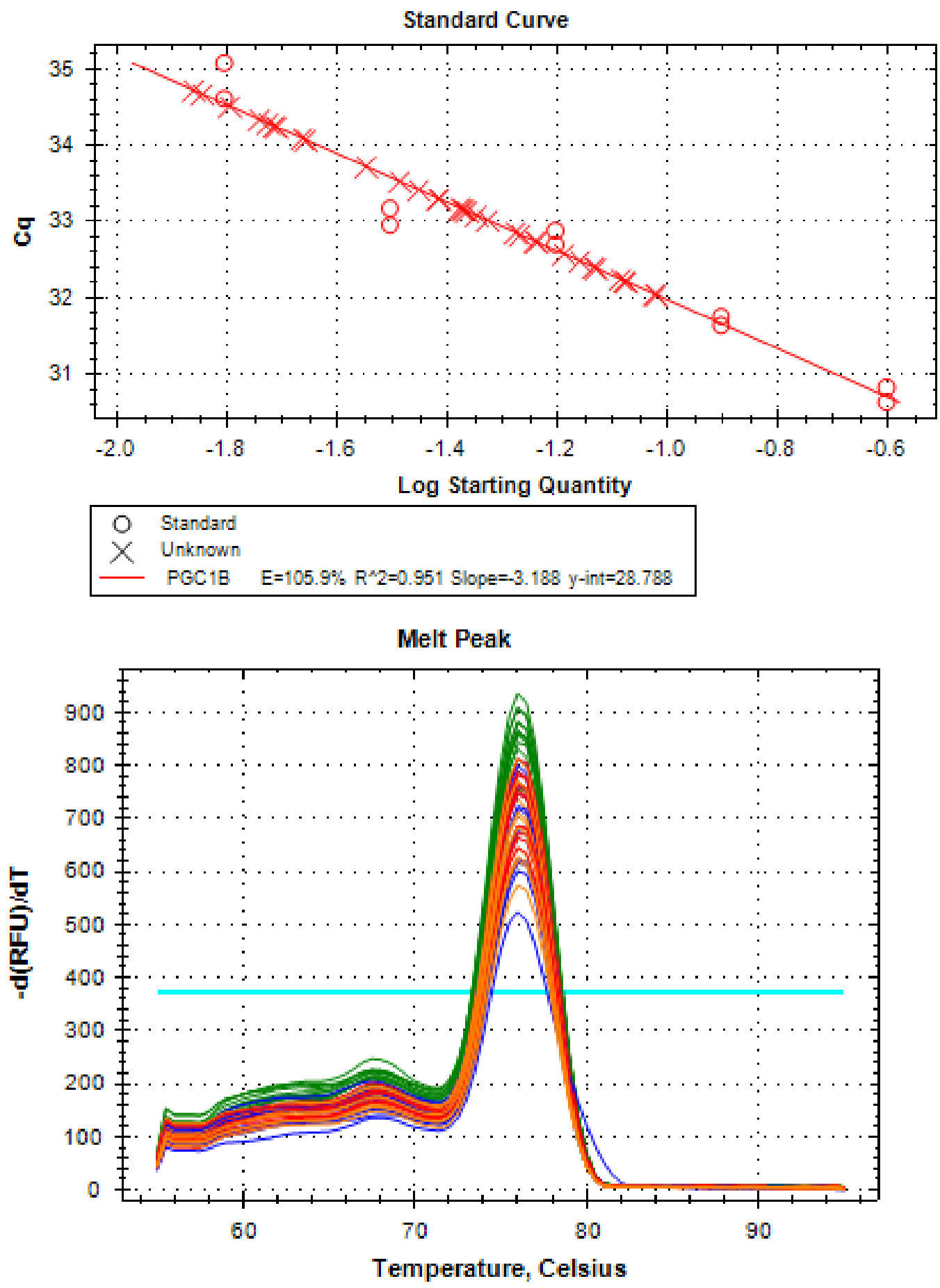
Once proper primer sets have been confirmed with a standard curve, cDNA of 'unknown' samples (individual biological replicates for control and dehydrated animals) undergo the same reaction. Relative expression values (with \% efficiency accounted for) from the gene of interest are normalized against the respective values from a reference gene - a target that is expected to be unchanged over all experimental conditions.

An example is provided below for the relative quantification of hifla mRNA in the lung of $X$. laevis under control and high dehydration:

\begin{tabular}{|c|c|c|c|c|c|c|c|c|c|}
\hline & Hif1a.L & RPL27 & Hif1a/RPL27 & Average & Std deviation & SEM & & & \\
\hline $\mathrm{C} 1$ & $1.28 \mathrm{E}-01$ & 6.57E-02 & $1.95 E+00$ & & & & & & \\
\hline $\mathrm{C} 2$ & $6.51 \mathrm{E}-02$ & $6.63 \mathrm{E}-02$ & $9.82 \mathrm{E}-01$ & & & & & & \\
\hline $\mathrm{C} 3$ & $6.43 E-02$ & $7.08 \mathrm{E}-02$ & $9.08 \mathrm{E}-01$ & & & & & & \\
\hline $\mathrm{C} 4$ & 7.21E-02 & $7.43 \mathrm{E}-02$ & $9.70 \mathrm{E}-01$ & & & & & & \\
\hline $\mathrm{C5}$ & 4.33E-02 & 7.85E-02 & $5.52 \mathrm{E}-01$ & & & & & & \\
\hline $\mathrm{C} 6$ & $6.71 E-02$ & 8.27E-02 & 8.11E-01 & $1.03 E+00$ & 0.520555708 & 0.2125 & & & \\
\hline $\mathrm{H} 1$ & $1.24 \mathrm{E}-01$ & $6.71 E-02$ & $1.85 E+00$ & & & & & Average & SEM \\
\hline $\mathrm{H} 2$ & 1.10E-01 & $8.36 \mathrm{E}-02$ & $1.32 E+00$ & & & & Control & $1.00 \mathrm{E}+00$ & 2.07E-01 \\
\hline H3 & $1.04 \mathrm{E}-01$ & $6.50 \mathrm{E}-02$ & $1.60 \mathrm{E}+00$ & & & & High & $1.57 \mathrm{E}+00$ & $1.06 \mathrm{E}-01$ \\
\hline $\mathrm{H} 4$ & $1.05 \mathrm{E}-01$ & $8.46 \mathrm{E}-02$ & $1.24 \mathrm{E}+00$ & & & & & & \\
\hline H5 & $1.36 \mathrm{E}-01$ & 7.72E-02 & $1.76 \mathrm{E}+00$ & & & & & & \\
\hline H6 & $1.08 \mathrm{E}-01$ & $5.56 \mathrm{E}-02$ & $1.94 \mathrm{E}+00$ & $1.62 E+00$ & 0.267531967 & 0.1092 & t-test & 0.031418 & $p<0.05$ \\
\hline
\end{tabular}




\section{Appendix F}

Reference gene selection 
Care must be given in choosing reference genes. Arbitrarily choosing a reference gene can result in false positives or false negatives if the expression of the gene is sensitive to the conditions studied (in this case, dehydration).

First, it is important to note that the concentration of the RNA samples, with which cDNA was synthesized, were standardized multiple times. As a result, variation between all samples can be expected, but not significant variation between experimental conditions.

Consider rpl27 expression in X. laevis lung in samples of both control and dehydration. In this case, a descriptive statistical test (e.g. $t$-test or one-way ANOVA) does not identify a statistically significant difference between the conditions, and suggests that this reference gene is appropriate:
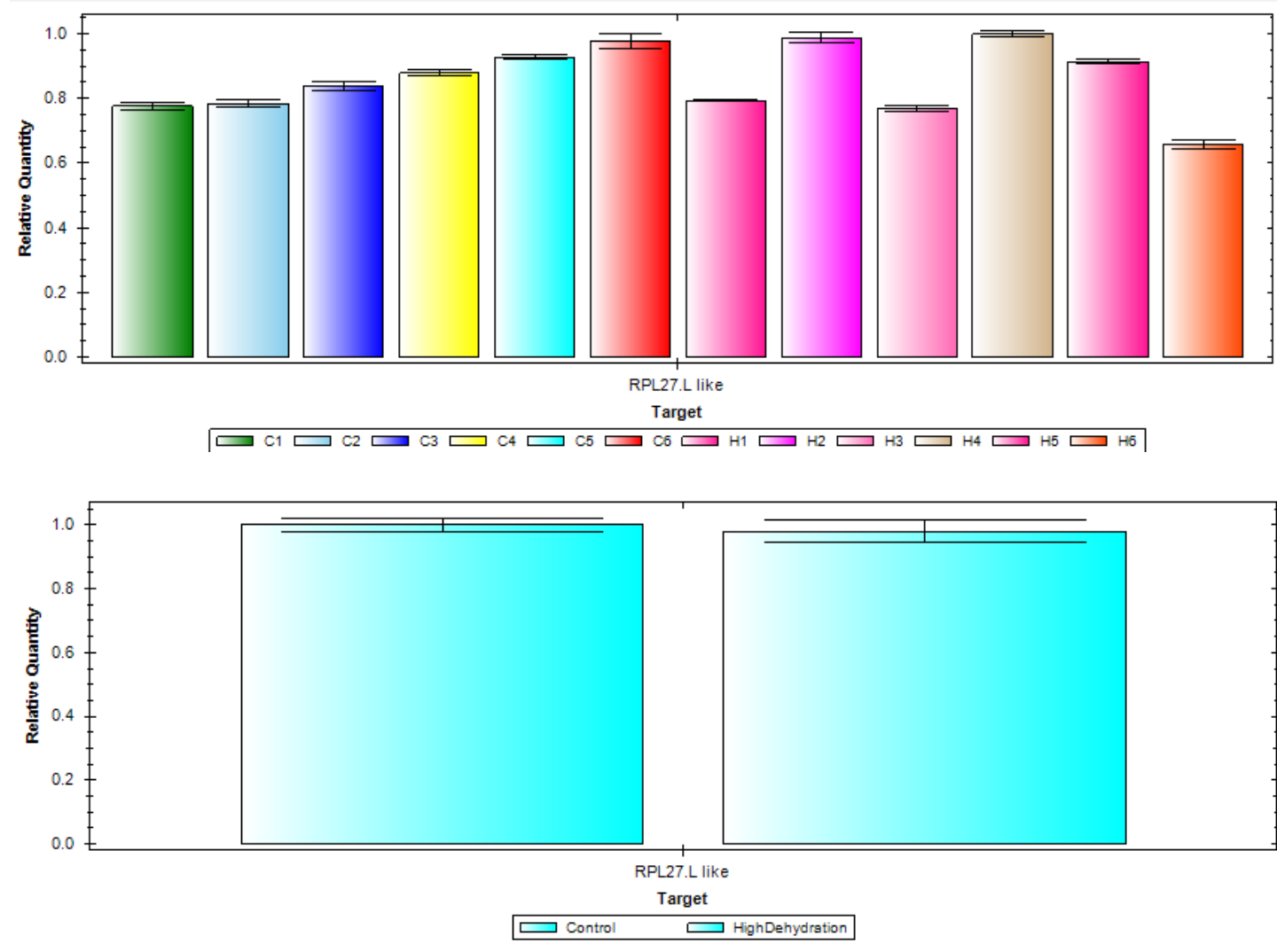
In five of the six tissues assessed in this thesis, rpl27 is deemed to be an appropriate reference gene. Descriptive statistics did not find any significant differences between control, medium and/or high dehydrated conditions. In heart, however, $r p l 27$ is not an appropriate gene, as it appears to increase during high dehydration:

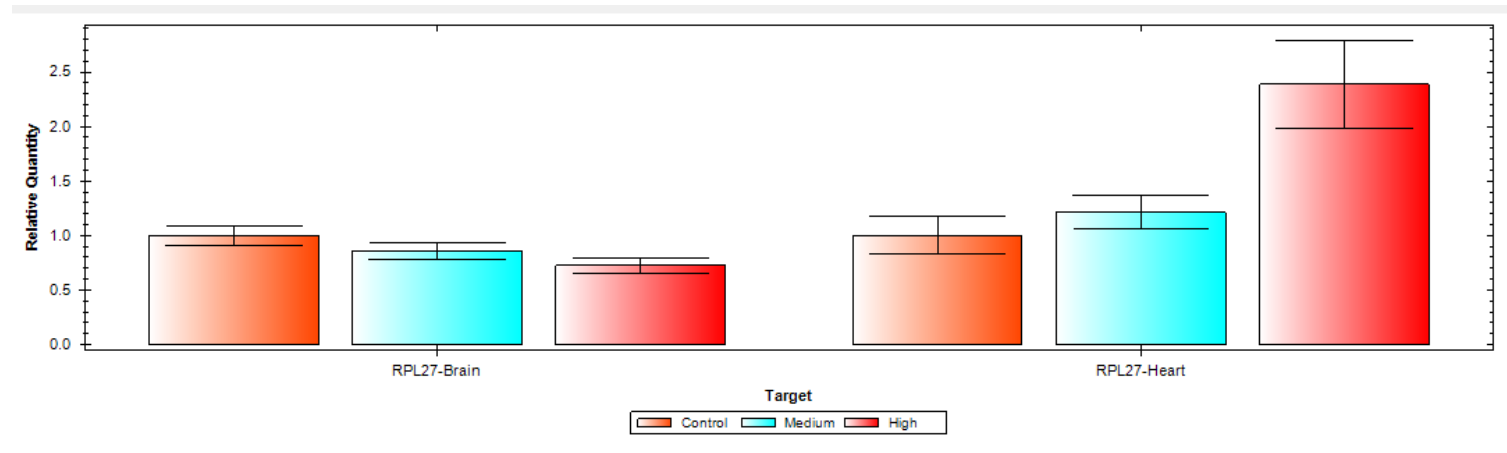

In this case of $X$. laevis heart, gapdh serves as a much better reference gene as there were no significant differences between control / medium dehydration / high dehydration conditions:
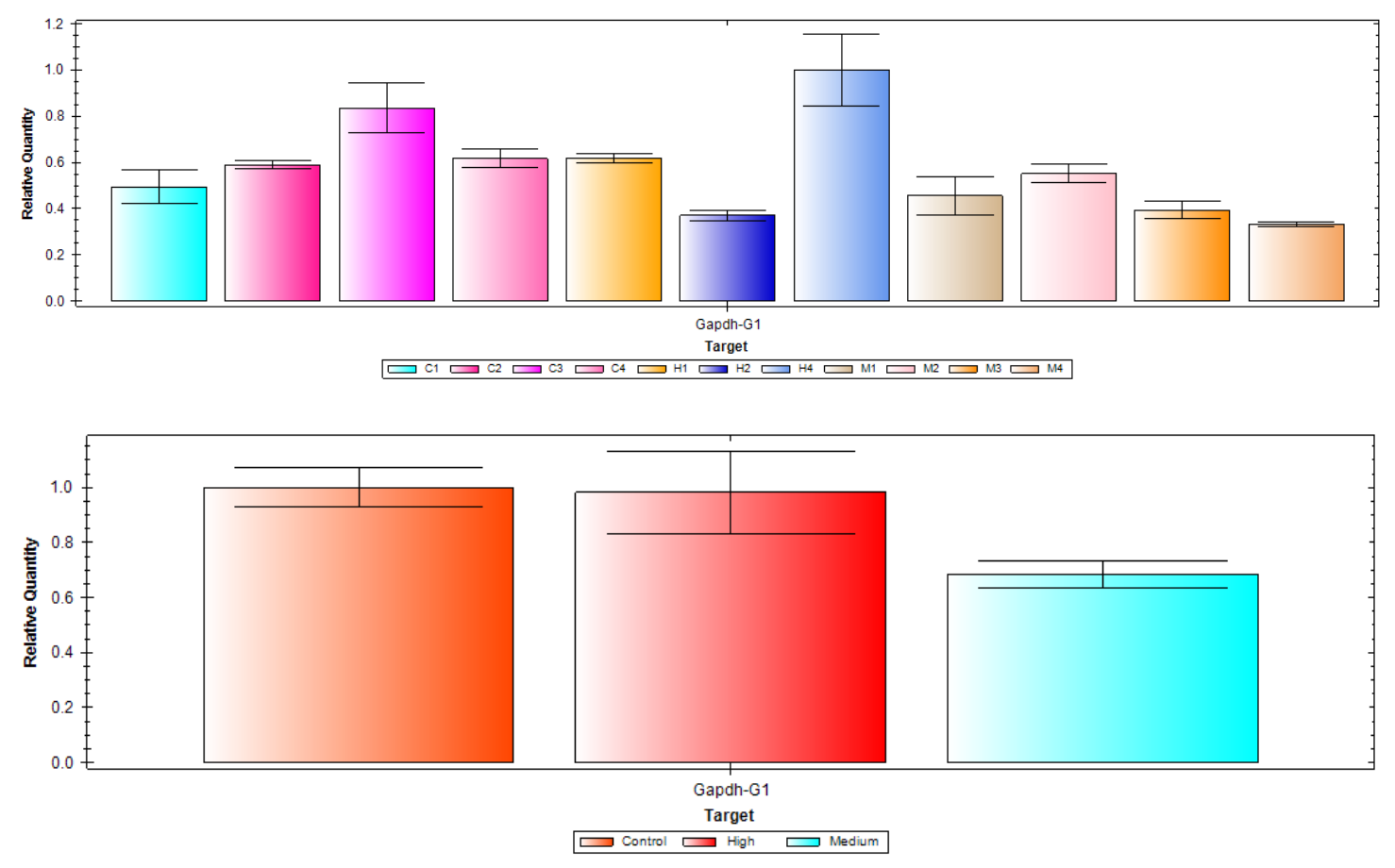


\section{Appendix G}

\section{Western immunoblot quantification}


In order to assure that there was no signal saturation, 3-point standard curves were run with pooled protein samples, created similarly to pooled cDNA samples. These samples were used to assure that quantification of immunoblots was able to detect a difference in protein amount. This also acted as a means of testing different antibodies, which may not always crossreact, particularly if they were not designed to crossreact with the intended protein target in multiple species.

Below is the result of a test immunoblot that was probing for SOD1 protein by loading $2 \mu \mathrm{g}, 6 \mu \mathrm{g}$, and $10 \mu \mathrm{g}$ of pooled $X$. laevis lung protein (control/medium dehydration/high dehydration). Quantification would be performed with $6 \mu \mathrm{g}$ protein loaded.

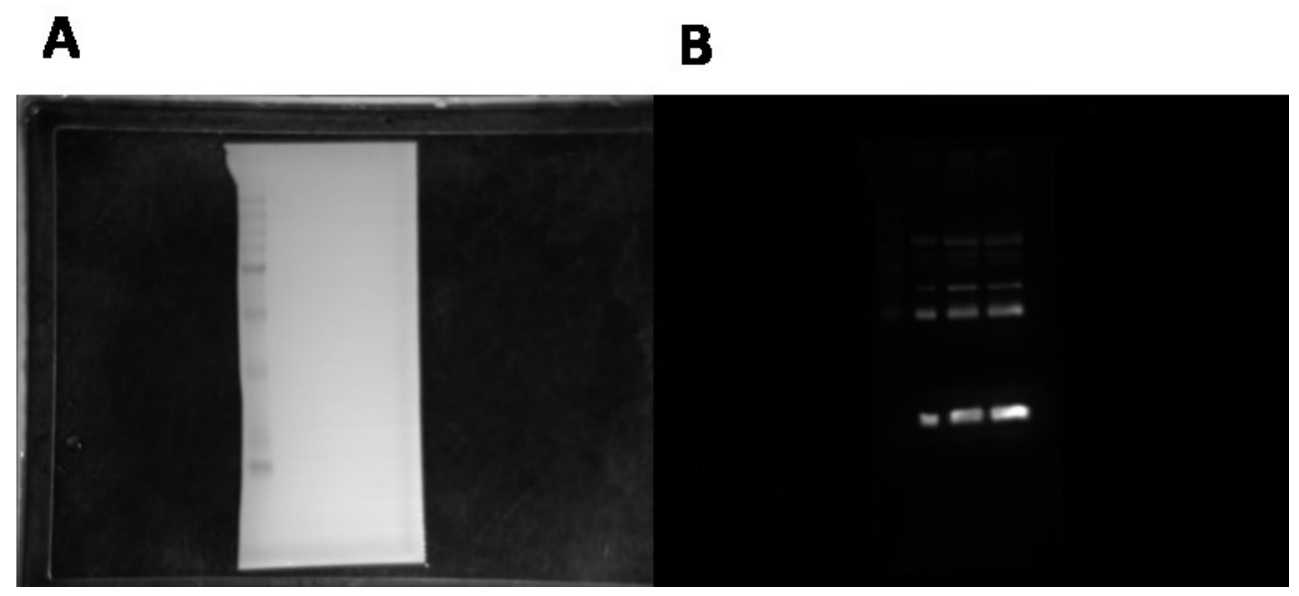

Figure G1. (A) An immunoblot probed with anti-SOD1 under white light $2 \mu \mathrm{g}, 6 \mu \mathrm{g}$, and $10 \mu \mathrm{g}$ of pooled $X$. laevis lung protein was loaded. (B) The same immunoblot undergoing a chemiluminescent reaction. The lowest three sets of bands show up at $\sim 15 \mathrm{kDa}$, where SOD1 was expected to appear.

The annotated $X$. laevis genome can be used to calculate an accurate molecular weight. Since the primary sequence of $X$. laevis SOD1 is known, it can be used to compute the theoretical molecular weight.

\section{superoxide dismutase [Cu-Zn] B [Xenopus laevis]}

NCBI Reference Sequence: NP_001080933.1

\section{GenPept Identical Proteins Graphics}

$>$ NP_001080933.1 superoxide dismutase [Cu-Zn] B [Xenopus laevis] MVKAVCVLAGSGDVKGVVHFEQQDEGAVSVEGKIEGLTDGLHGFHIHVFGDNTNGCMSAGSHFNPENKNH GAPGDTDRHVGDLGNVTAEGGVAQFKITDSLISLKGPNSIIGRTAVVHEKADDLGKGGNDESLKTGNAGG RLACGVIGYSP 


\section{Compute $\mathrm{pl} / \mathrm{Mw}$}

Theoretical $\mathrm{pl} / \mathrm{Mw}$ (average) for the user-entered sequence:

\begin{tabular}{|c|c|c|c|c|c|}
\hline $1 \underline{0}$ & $2 \underline{\underline{\theta}}$ & $3 \underline{0}$ & $4 \underline{0}$ & $5 \underline{\underline{\theta}}$ & $6 \underline{0}$ \\
\hline MVKAVCVLAG & SGDVKGVVHF & EQQDEGAVSV & EGKIEGLTDG & LHGFHIHVFG & DNTNGCMSAG \\
\hline $7 \underline{0}$ & $8 \underline{0}$ & 90 & 100 & 110 & 120 \\
\hline SHFNPENKNH & GAPGDTDRHV & GDLGNVTAEG & GVAQFKITDS & LISLKGPNSI & IGRTAVVHEK \\
\hline $13 \underline{0}$ & $14 \underline{\underline{\theta}}$ & $15 \underline{Q}$ & & & \\
\hline ADDLGKGGND & ESLKTGNAGG & RLACGVIGYS & r & & \\
\hline
\end{tabular}

Theoretical pl/Mw: $5.50 / 15418.07$

Protein sequence analyses were done with Clustal Omega and $\mathrm{pI} / \mathrm{Mw}$ predictors for all proteins analyzed in this theses, including the Sirtuins and proteins that regulate antioxidants and metabolism. 
A

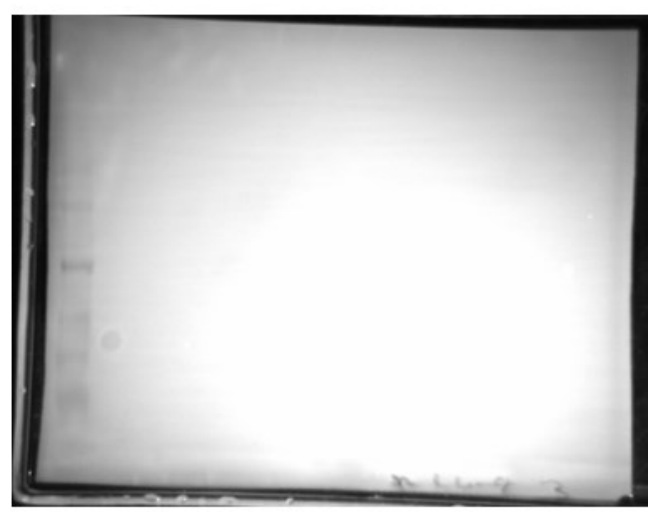

B

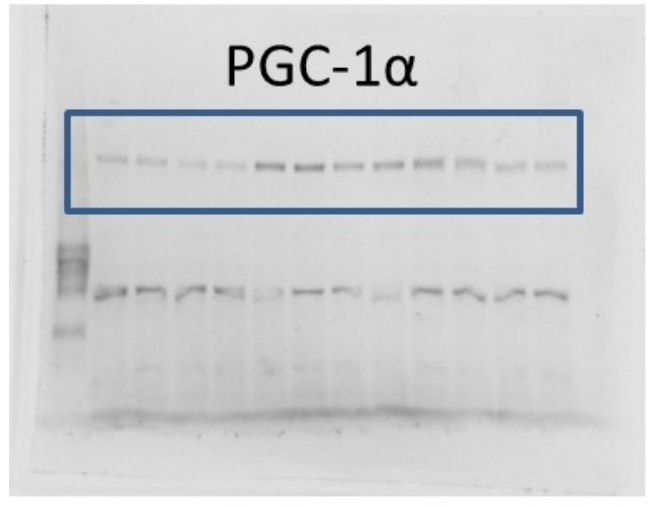

C

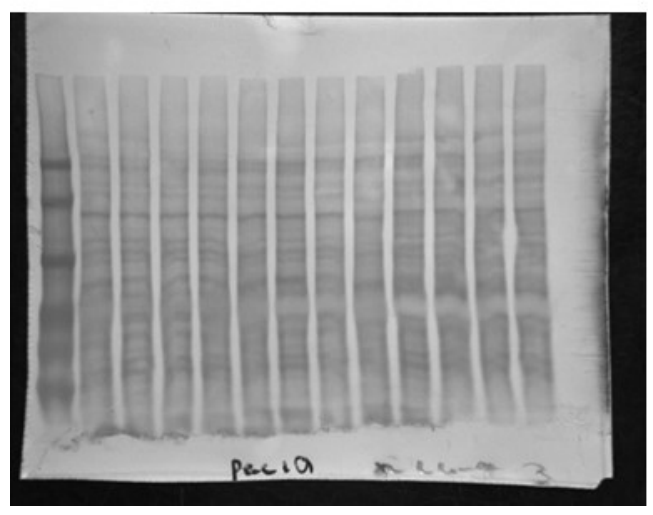

Figure G2. A Western immunoblot run for quantification of PGC-1 $\alpha$ protein in the lung of $X$. laevis under control, medium dehydration, and high dehydration conditions imaged under (A) white light, (B) chemiluminescent exposure, and (C) white light with Coomassie Blue staining. For quantification, PGC-1 $\alpha$ signal from each lane (B) was normalized against the total protein loaded of the respective lane, represented by Coomassie staining. 
Appendix H

Antibody list 
Table H1. Antibody supplier and catalog number.

\begin{tabular}{cccc}
\hline Antibody & Type & Company & Catalog \# \\
\hline SIRT1 & Total & Cell Signaling & 8469 \\
SIRT2 & Total & Santa Cruz & sc-20966 \\
SIRT3 & Total & GeneTex & GTX115701 \\
SIRT6 & Total & Active Motif & 39912 \\
PGC-1 $\alpha$ & Total & Santa Cruz & sc-13067 \\
FoxO1 & Total & GeneTex & GTX110724 \\
FoxO1 & p-Ser256 & Cell Signaling & 9461 \\
FoxO1 & p-Ser319 & Cell Signaling & 2487 \\
FoxO3 & Total & GeneTex & GTX100277 \\
FoxO3 & p-Ser253 & Cell Signaling & 9466 \\
Catalase & Total & Genscript & A01188 \\
HO-1 & Total & StressGen & ADI-SPA-896-D \\
SOD1 & Total & Genscript & A01005 \\
SOD2 & Total & Biosciences & 611580 \\
SOD2 & Ac-K68 & Abcam & ab137037 \\
MEF2A & Total & GeneTex & GTX50397 \\
MEF2C & Total & GeneTex & GTX105433 \\
Hif-1 $\alpha$ & Total & ABclonal & A11945 \\
Histone H3 & Total & Cell Signaling & 9715 \\
\hline
\end{tabular}




\section{Appendix I}

Western blot conditions 
Table I1. Western blot targets and conditions.

\begin{tabular}{|c|c|c|c|c|c|c|c|}
\hline Antibody & Type & $\begin{array}{l}\text { Gel } \\
(\%)\end{array}$ & $\begin{array}{c}\text { MW } \\
\text { (kDa) }\end{array}$ & $\begin{array}{l}\text { [Primary } \\
\text { antibody] }\end{array}$ & $\begin{array}{c}\text { Reactivity } \\
\text { (Anti-) }\end{array}$ & $\begin{array}{c}\text { [Secondary } \\
\text { antibody] }\end{array}$ & Transfer \\
\hline SIRT1 & Total & 8 & 110 & $1: 1000$ & Mouse & $1: 2000$ & $\begin{array}{l}160 \mathrm{~mA} ; \\
90 \mathrm{~min}\end{array}$ \\
\hline SIRT2 & Total & 10 & 43 & $1: 1000$ & Rabbit & 1:4000 & $\begin{array}{c}160 \mathrm{~mA} \\
90 \mathrm{~min}\end{array}$ \\
\hline SIRT3 & Total & 10 & 30 & 1:1000 & Rabbit & $1: 4000$ & $\begin{array}{c}160 \mathrm{~mA} \\
90 \mathrm{~min}\end{array}$ \\
\hline SIRT6 & Total & 10 & 37 & $1: 1000$ & Rabbit & 1:4000 & $\begin{array}{c}160 \mathrm{~mA} \\
90 \mathrm{~min}\end{array}$ \\
\hline PGC-1 $\alpha$ & Total & 8 & 87 & $1: 1000$ & Rabbit & 1:8000 & $\begin{array}{c}160 \mathrm{~mA} \\
90 \mathrm{~min}\end{array}$ \\
\hline FoxO1 & Total & 8 & 68 & $1: 1000$ & Rabbit & 1:8000 & $\begin{array}{c}160 \mathrm{~mA} \\
90 \mathrm{~min}\end{array}$ \\
\hline FoxO1 & p-Ser 256 & 8 & 68 & $1: 1000$ & Rabbit & 1:4000 & $\begin{array}{c}160 \mathrm{~mA} \\
90 \mathrm{~min}\end{array}$ \\
\hline FoxO1 & p-Ser319 & 8 & 68 & $1: 1000$ & Rabbit & 1:4000 & $\begin{array}{c}160 \mathrm{~mA} \\
90 \mathrm{~min}\end{array}$ \\
\hline FoxO3 & Total & 8 & 70 & 1:1000 & Rabbit & $1: 8000$ & $\begin{array}{c}160 \mathrm{~mA} \\
90 \mathrm{~min}\end{array}$ \\
\hline FoxO3 & p-Ser 253 & 8 & 70 & $1: 1000$ & Rabbit & 1:4000 & $\begin{array}{l}160 \mathrm{~mA} \\
90 \mathrm{~min}\end{array}$ \\
\hline Catalase & Total & 8 & 60 & 1:1000 & Rabbit & 1:8000 & $\begin{array}{l}160 \mathrm{~mA} \\
90 \mathrm{~min}\end{array}$ \\
\hline HO-1 & Total & 15 & 33 & 1:1000 & Rabbit & 1:8000 & $\begin{array}{l}160 \mathrm{~mA} \\
90 \mathrm{~min}\end{array}$ \\
\hline SOD1 & Total & 15 & 15 & $1: 1000$ & Rabbit & 1:8000 & $\begin{array}{c}30 \mathrm{~V} ; 100 \\
\min \end{array}$ \\
\hline SOD2 & Total & 15 & 25 & $1: 1000$ & Mouse & 1:4000 & $\begin{array}{c}30 \mathrm{~V} ; 100 \\
\min \end{array}$ \\
\hline SOD2 & Ac-K68 & 15 & 25 & $1: 1000$ & Rabbit & 1:8000 & $\begin{array}{c}30 \mathrm{~V} ; 100 \\
\min \end{array}$ \\
\hline MEF2A & Total & 10 & 56 & 1:1000 & Rabbit & 1:8000 & $\begin{array}{l}160 \mathrm{~mA} \\
90 \mathrm{~min}\end{array}$ \\
\hline MEF2C & Total & 10 & 46 & $1: 1000$ & Rabbit & 1:8000 & $\begin{array}{l}160 \mathrm{~mA} \\
90 \mathrm{~min}\end{array}$ \\
\hline Hif- $1 \alpha$ & Total & 8 & 90 & 1:1000 & Rabbit & 1:8000 & $\begin{array}{c}160 \mathrm{~mA} \\
90 \mathrm{~min}\end{array}$ \\
\hline $\begin{array}{c}\text { Histone } \\
\text { H3 }\end{array}$ & Total & 15 & 18 & $1: 1000$ & Rabbit & $1: 8000$ & $\begin{array}{c}30 \mathrm{~V} ; 100 \\
\min \end{array}$ \\
\hline
\end{tabular}


Appendix J

List of primers 
Table J1. List of primers used for RT-qPCR.

\begin{tabular}{|c|c|c|}
\hline Gene & Forward (5' - 3') & Reverse (5' - 3') \\
\hline cat & CAGCGGCTATGTGAGAACCT & TGGGTCTGGCACATTCCTTC \\
\hline cpsl & TGGCTTCAAAGGCTACTGGG & CGTTGTCTTCCCTGACACGA \\
\hline cptla & AATGGAGCAGGGTCTCCTCA & TGCCTGACTTTCGTGAAGGAT \\
\hline cpt $1 b$ & ATGTCCAGTCGGCAACCAAT & CTGGAATTCCTCAGCCAGGG \\
\hline foxol & TTTGTGCTGCTCTTGGACCTC & CCCTTAGATTCTCTGCTGCCTT \\
\hline foxo 3 & GCTAGGCCTCAACCATTCAC & GCAGTTGTGTTTTCCAGCATGA \\
\hline gapdh & GCCGCCATTAAGACTGCATCA & TGGAGACAACCTGGTCTTGTG \\
\hline glut 4 & ATCTGTGTGGCCCTTACGTG & CTCATCGAAGGATCTGCCCC \\
\hline hifla & ACCGCACTACTTCAGTTTGCT & TGTTCTTGCACGTTTTTCCCC \\
\hline$h k$ & GACTCCGCACCGAACAAAAC & ACTGAGGAGTGCACGTTTCT \\
\hline$h n f 3 b$ & CGGACAAGCCTGGAAAAGGT & CTTGAAGCGTTTCTGTCGCC \\
\hline hnf4a & TCTTTGGGAAAGCGTGGGAA & CAGCTGGGACCGGAACTTG \\
\hline ho-1 & GGCCAAAACAGCATTCCTGC & CTGTTGTTCTCTCTGTTGTCTGT \\
\hline lcad & GAAGTGCAGCAGAGTTCCCT & TAGCAACGATTCCCTCTGGC \\
\hline mcad & CGAACACCTCGGCAAGTACA & GGCTCACACTACTGAAAGTGC \\
\hline$m e f 2 a$ & AGGGAGTCGTAGGGTGTTGT & ACAAGCTAAAATCAGTTCTCTGTCT \\
\hline$m e f 2 c$ & CATTCGCTCGGCCGTCTGT & ATCCCAAGTCAGTCCAATCGC \\
\hline otc & TGTGCAGTTAAAAGGCGGGA & TCAGATCAGTTGCCACCCAC \\
\hline ppara & TTTGACCCCTTTGGACGAGG & CCGAAGAACTGATGCTCGGA \\
\hline$p d k 1$ & TAAAAGGGAAGCTCAGCCTGT & TGAAATGTGGTTCTCCCTGCC \\
\hline$p d k 3$ & GACAAAACCGATGCCTTGCC & ATTTTCCTCAAGGGCACCCC \\
\hline$p d k 4$ & CAGCCCACCCAAAACACATC & GGATCATTTCCATTCGTCTGCT \\
\hline$p f k$ & TGTGTTGAGAGCCTCGGTTG & AGCAGTACTGTGGTGTGACAAA \\
\hline pgcla & AATCTTCTGTGCCACCTGCT & CCAATTGCCACGGTCGTTTT \\
\hline pgclb & ACTATGCACTGTAGTGGGGTG & GCCCAGTAATGGTTCATGGCT \\
\hline rpl27 & GAGTTGGAGTGAAAATGGGCA & TCACAATGACAGCTTTGCGG \\
\hline scad & GTAACGGATATGCCTGCCGA & AGCCTCTGGATCTCACTCGT \\
\hline sirt1 & AAAATCAATAAACCCACTTGGCA & CGGAAAGCACAAGATGAGTGTG \\
\hline sirt2 & AGAGCTGAGAGTGCAGTTTAGG & ACTCCAGATGGGCTAACGTG \\
\hline sirt3 & ACCACCCAAACACTGCTCAT & CCGTGAGCTTCCACCAACTT \\
\hline sirt4 & GCAGGTGGCAAACTTTCAGG & ACACAAAACCTCGGCTCACA \\
\hline sirt5 & TTACATCGCAAGGCAGGCTC & TTTCCGTCAAGAGCTGGACAA \\
\hline sirt6 & CACACCGTGCTGTATTTGCT & TCGGTTTCCACTTTGCGGAT \\
\hline sirt7 & GGTGTGTCTGATCCTGCGAA & CATCCAACACCTCCTGTTCCC \\
\hline sodl & AACAGGACGATGGTGACGTG & ACACGTGGATATGGAAGCCG \\
\hline $\operatorname{sod} 2$ & TCAACTCTCAGAGCACATACAG & AACTAACATTGCTGCTGCCTT \\
\hline vegf & CTTCAAACCATCCTGCGTGC & GTGACTGTGCTGCTGAAAGC \\
\hline
\end{tabular}

